\title{
AN INTERFEROMETRIC STUDY OF UNSTEADY PASSING SHOCK FLOW IN A TURBINE CASCADE
}

\author{
$\mathrm{By}$
}

Angela L. Wesner

A DISSERTATION SUBMITTED TO THE FACULTY OF VIRGINIA POLYTECHNIC INSTITUTE AND STATE UNIVERSITY

IN PARTIAL FULFILLMENT OF THE REQUIREMENTS FOR THE DEGREE OF DOCTOR OF PHILOSOPHY

IN

Aerospace Engineering

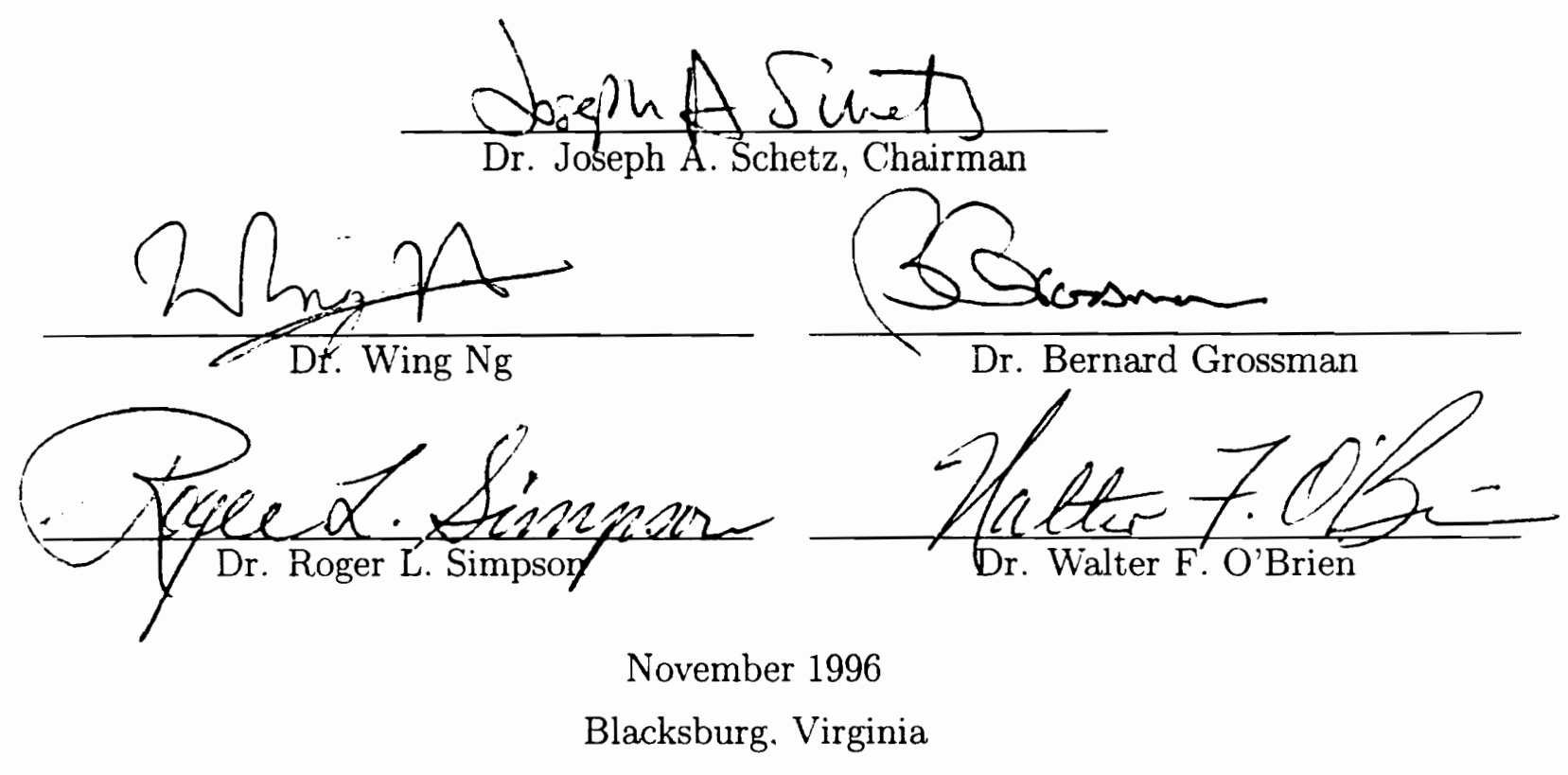

Keywords: interferometric, unsteady, shock, turbine, cascade, transition and turbulence 


\title{
AN INTERFEROMETRIC STUDY OF UNSTEADY PASSING SHOCK FLOW IN A TURBINE CASCADE
}

by

\author{
Angela L. Wesner \\ Committee Chairman: Joseph A. Schetz \\ Aerospace Engineering
}

(ABSTRACT)

A single-plate interferometric system was developed for the evaluation of unsteady flow generated by a passing shock in a linear transonic turbine cascade. A $15 \mathrm{~mW}$ Helium-Neon laser was used as the light source. An intensified charge-coupled device (CCD) was used to capture images, allowing the required 100 ns shutter times to "freeze" the passing shock. High frequency Kulite pressure transducers and miniature hot wire probes were used to capture the required known density field for the quantitative evaluation of the flowfield. Variations in the density field data as the shock passes upstream of the studied blade passage are presented, showing the capabilities of this system and detailing the unsteady flow field. The automation of the interferogram interpretation is presented. Image analysis techniques used include adaptive binarization and Hilditch line thinning. Further areas for improvement are also discussed.

In addition to the interferometric study, a boundary layer transition study was 
also undertaken. Magnified boundary layer spark shadowgraphs were taken on both the pressure and suction surfaces of the turbine blade. Flow conditions including low $(<1.0 \%)$ and high $(5 \%)$ free stream turbulence values were investigated. The transition location was found to be dependent not only on the level of turbulence but also on the turbulence characteristics. Further, an investigation was made into the application of hydrodynamic stability theory to turbine blade flows. The results of this evaluation were used in the determination of appropriate length scales for tailoring free stream turbulence to more effectively induce suction surface transition. Little instability and no transition was calculated for the blade suction surface in agreement with the shadowgraph results. Free stream turbulence with frequencies near $40 \mathrm{kHz}$ were found to most affect the instabilities in the boundary layer. Tailored free stream turbulence was found to produce transition on the blade suction surface. 


\section{Acknowledgements}

Throughout my three plus years vorking on this project, many individuals have made great contributions to this body of work and provided me with immeasurable support and guidance. First and foremost among these is my advisor and committee chair, Dr. J. A. Schetz. I would like to thank him for all of his encouragement and confidence.

I would also like to thank the other two project heads, Dr. W. Ng and Dr. T. Diller. I gained a great deal of insight into turbomachinary from their thoughtful comments about the relevance of my work.

I would like to thank Dr. B. Grossman, Dr. R. Simpson and Dr. W. O'Brien for serving on my committee.

When I first began this project, Tibor Kiss, whose work I am expanding here, was kind enough to "show me the ropes". This was a tremendous help to me, for not only did I get a running start on the work, but a large amount of the anxiety I had about working in a wind tunnel environment was dissipated. I am grateful to him for all the patience he showed me while I was learning the basics of his optical system.

A number of other graduate students were also working on different aspects of this project. I would like to take this opportunity to extend them my undying thanks. To Dave Holmberg and Terry Reid, you were always willing to offer suggestions from your vast storehouses of knowledge about the tunnel, specifically, and life, in general. Thanks also to Missy Fasold and Loren Johnson, who were great friends, supporting me in everything that I did and willing to help me with almost anything; I had only to ask. And finally, thanks to Drew and Hume, who were always there to inject a bit of timely humor into my day.

To Steve Lepera and Dr. U. Vandsburger, thank you for all of the help with the 
laser and the camera.

To all of my parents, John and Beverly Judkins and Darrell and Marilyn Wesner, who taught me that you never really truly appreciate something unless you work hard to get it; thank you. Without this most important lesson, I never would have had the perserverance to complete the work you are about to read. And to my Uncle Alan and Aunt Joyce, thanks for taking care of the window emergency. I am very fortunate to have family like you, who are willing to help without hesitation, at a moments notice.

When deadlines got tight, the guys in the shop and the office were always willing to put other things aside and finish up my work. Many thanks to Gary Stafford, Bruce, Kent, Greg, Jerry Lucas, Timmy, Clark, Wanda Foushee and Mrs. Willams.

I would also like to thank the National Science Foundation for their support of this work.

I know I am not the most pleasant person to be around when I am engrossed in some project, so I want to thank Lt. Neil Thomas for sticking with me through all of these years. When things got rough, you were always there with a shoulder for me to lean on.

I am eternally grateful to my assistant, Hank Grabowski. My thanks could never be enough to compensate for all of the hard work he put in to this research over this last summer. 
To the Dance 


\section{Contents}

$\begin{array}{ll}\text { Abstract } & \text { ii }\end{array}$

Acknowledgements iv

Nomenclature $\quad$ xvi

1 Introduction 1

1.1 Literature Review . . . . . . . . . . . . . . . . . . . . 5 5

2 Transonic Tunnel Design 10

2.1 Test Section and Blade Design . . . . . . . . . . . . . . . . 12

2.1.1 Cascade Flow Characteristics . . . . . . . . . . . 13

2.1.2 Blade Static Pressure Measurements . . . . . . . . . . 17

2.2 Optical Window Construction . . . . . . . . . . . . . . 21

2.3 Shock Wave Generation . . . . . . . . . . . . . . . . . 23

2.4 Heated Flow . . . . . . . . . . . . . . . . . . . . . 24

3 Transition Study 28

3.1 Shadowgraph Study . . . . . . . . . . . . . . . . . 28

3.1 .1 Apparatus . . . . . . . . . . . . . . . . 29

3.1 .2 Interpretation of Shadowgraphs . . . . . . . . . . 31

3.2 Boundary Layer Analysis . . . . . . . . . . . . . . . . . . 31

3.2 .1 Boundary Layer Code . . . . . . . . . . . . . . . . 36

$3.2 .2 \quad$ Boundary Layer Code Input . . . . . . . . . . . . . . . 39 
3.2.3 Boundary Layer Results . . . . . . . . . . . . . . . . . 40

3.3 Stability Analysis . . . . . . . . . . . . . . . . . . . 43

3.3.1 Hydrodynamic Stability Theory Background . . . . . . . . 43

$3.3 .2 \quad$ Stability Code . . . . . . . . . . . . . . . . . 47

3.3.3 Stability Calculation Results . . . . . . . . . . . . . . 51

3.4 Free Stream Turbulence Effects . . . . . . . . . . . . . . . 53

3.4 .1 Grid Design . . . . . . . . . . . . . . . . . 53

3.4.2 Shadowgraph Results with FST . . . . . . . . . . 67

4 Interferometric System Development $\quad 72$

4.1 Introduction . . . . . . . . . . . . . . . . . . . 72

4.1 .1 Historical Background . . . . . . . . . . . . 72

4.1 .2 Fundamentals . . . . . . . . . . . . . . . . . . . 73

4.1 .3 Interpretation . . . . . . . . . . . . . . . . 77

4.2 Previous Steady Flow Interferometric System at Virginia Tech . . . . 77

4.3 Required System Improvements for Unsteady Flows . . . . . . . . . 79

4.4 Additional Measurement Instruments . . . . . . . . . . . . . . . 83

4.5 Component Selection . . . . . . . . . . . . . . . . . 85

4.5.1 Optical Components . . . . . . . . . . . . . . . 85

4.5.2 High Frequency Pressure Transducers . . . . . . . . . . 85

4.5 .3 Passage Hot-Wire . . . . . . . . . . . . . . . . . 87

$4.5 .4 \quad$ CCD Camera . . . . . . . . . . . . . . . . . . 93

$4.5 .5 \quad$ Laser . . . . . . . . . . . . . . . . . . . . . 96 96

4.5.6 Timing Delay Circuit . . . . . . . . . . . . . . . . . 98

4.5.7 Strain Gage Balance and Amplifier . . . . . . . . . . . 98

4.5 .8 Waveform Recorder . . . . . . . . . . . . . . . 100

4.6 Problem Issues . . . . . . . . . . . . . . . . . . . . . . . 101

4.7 Human Factors . . . . . . . . . . . . . . . . . . . . . . . . 102

4.8 Future Improvements . . . . . . . . . . . . . . . . . . . . . . 102

5 Data Collection and Reduction $\quad 104$

5.1 Data Collection . . . . . . . . . . . . . . . . . . . . . . . . 104 
5.1.1 Steady, Unheated and Heated Reference Density Field Collection104

5.1.2 Upstream, Unsteady, Unheated Reference Density Field Collec-

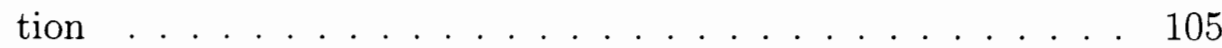

5.1.3 Unsteady Interferograms . . . . . . . . . . . . . . . . . 106

5.1.4 Calibration of the Hotwire . . . . . . . . . . . . . . . 108

5.2 Data Reduction . . . . . . . . . . . . . . . . . . . . . . . . . 110

5.2.1 Upstream, Steady, Heated and Unheated Reference Density Fields110

5.2.2 Upstream, Unsteady, Unheated Reference Density Fields . . . 112

5.2.3 Unsteady Density Data Correction . . . . . . . . . . . . 116

5.3 Digital Image Processing . . . . . . . . . . . . . . . . . . . 117

5.3 .1 Stretching . . . . . . . . . . . . . . . . . 117

5.3 .2 Low-Pass Filtering . . . . . . . . . . . . . . . 133

5.3 .3 Adaptive Binarization . . . . . . . . . . . . . . . 133

5.3 .4 Line Thinning . . . . . . . . . . . . . . . . . . . 134

5.4 Uncertainties . . . . . . . . . . . . . . . . . . . . . . 134

6 Results 139

$\begin{array}{lll}7 & \text { Conclusions } & 167\end{array}$

$\begin{array}{ll}\text { A QR Algorithm } & 175\end{array}$

$\begin{array}{ll}\text { B Avg01.cpp } & 178\end{array}$

$\begin{array}{ll}C \text { ensavg.cpp } & 190\end{array}$

$\begin{array}{ll}\text { D Fdz.m } & 200\end{array}$

$\begin{array}{ll}\text { E FringeFinal.cpp } & 201\end{array}$

$\begin{array}{lll}\text { F } \text { Sitc.cpp } & 216\end{array}$

$\begin{array}{ll}\text { G Flip.cpp } & 220\end{array}$

H ProcFin.cpp $\quad 223$ 
I Raw Interferometric Data $\quad 248$

Vita

249 


\section{List of Figures}

1.1 Unsteady Shock Wave Passing . . . . . . . . . . . . . . 3

2.1 Virginia Tech Transonic Cascade Wind Tunnel . . . . . . . . . . . . . 11

2.2 Virginia Tech Transonic Cascade Test Section . . . . . . . . . . . 14

2.3 Transonic Turbine Blade Profile . . . . . . . . . . . . . . . 15

2.4 Transonic Turbine Cascade . . . . . . . . . . . . . . . 16

2.5 Transonic Turbine Cascade Shadowgraph . . . . . . . . . . . . . 18

2.6 Oil Flow Visualization of Cascade Endwall . . . . . . . . . . . . . . 19

2.7 Oil Flow Visualization of Single Blade Suction Surface . . . . . . 20

2.8 Isentropic Mach Number Distribution on Blade Surface . . . . . . . 22

2.9 Shock-Tube Construction [Doughty,1994] . . . . . . . . . . . . 25

2.10 Shock-Shaper Construction $[$ Doughty,1994] . . . . . . . . . . . 26

2.11 Passing Shock Orientation Within the Test Section . . . . . . . . 26

3.1 Magnified Spark-Shadowgraph Images of Boundary Layer, Low FST (A) 32

3.2 Magnified Spark-Shadowgraph Images of Boundary Layer, Low FST (B) 33

3.3 Magnified Spark-Shadowgraph Images of Boundary Layer, Low FST (C) 34

3.4 Typical Laminar and Turbulent Boundary-Layer Profiles [Holder, 1956] 35

3.5 Steady Surface Isentropic Mach Number Distribution - All Cases . . . 41

3.6 Steady Boundary Layer Thickness - All Cases _ . . . . . . . . . . . 42

3.7 Steady Pressure Gradient Parameter Distribution - All Cases . . . . . 42

3.8 Steady, Adiabatic Wall Boundary Layer Michel Plot . . . . . . . . . . 44

3.9 Steady Momentum Thickness Reynolds Number - All Cases . . . . . 44

3.10 Steady Wall Skin Friction Distribution - All Cases . . . . . . . . . . 55 
3.11 Comparison of Spatial Amplification Rates for a Boundary Layer Disturbance of $25 \mathrm{kHz}$ (Note negative $\alpha$ implies amplification) . . . . . 56

3.12 Comparison of Spatial Amplification Rates for a Boundary Layer Disturbance of $30 \mathrm{kHz}$ (Note negative $\alpha$ implies amplification) . . . . 57

3.13 Comparison of Spatial Amplification Rates for a Boundary Layer Disturbance of $35 \mathrm{kHz}$ (Note negative $\alpha$ implies amplification) . . . . 58

3.14 Comparison of Spatial Amplification Rates for a Boundary Layer Disturbance of $40 \mathrm{kHz}$ (Note negative $\alpha$ implies amplification) . . . . .

3.15 Comparison of Spatial Amplification Rates for a Boundary Layer Disturbance of $45 \mathrm{kHz}$ (Note negative $\alpha$ implies amplification) . . . . . .

3.16 Comparison of Spatial Amplification Rates for a Boundary Layer Disturbance of $50 \mathrm{kHz}$ (Note negative $\alpha$ implies amplification) . . . . .

3.17 Comparison of Spatial Amplification Rates for a Boundary Layer Disturbance of $55 \mathrm{kHz}$ (Note negative $\alpha$ implies amplification) . . . . .

3.18 Comparison of Spatial Amplification Rates for a Boundary Layer Disturbance of $60 \mathrm{kHz}$ (Note negative $\alpha$ implies amplification) . . . . . .

3.19 Comparison of Spatial Amplification Rates for a Boundary Layer Disturbance of $65 \mathrm{kHz}$ (Note negative $\alpha$ implies amplification) . . . . .

3.20 Comparison of Disturbance Frequency Effects on the Spatial Amplification Rates for the Adiabatic Wall Case (Note negative $\alpha$ implies amplification) $\ldots \ldots \ldots \ldots \ldots \ldots \ldots \ldots$

3.21 Combined Influence of Streamwise Pressure Gradient and Free Stream Turbulence Intensity on Boundary Layer Transition . . . . . . . 66

3.22 Magnified Spark-Shadowgraph of Boundary Layer Transition with Grid $\# 2$ at $3.56 \mathrm{~cm} \ldots \ldots \ldots \ldots \ldots \ldots \ldots \ldots$

3.23 Magnified Spark-Shadowgraph of Boundary Layer Transition with Grid

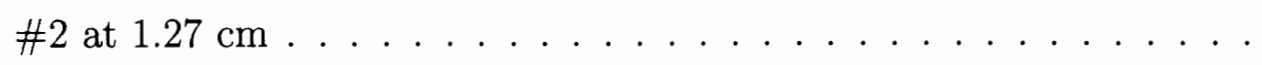

3.24 Magnified Spark-Shadowgraph of Boundary Layer Transition with Grid

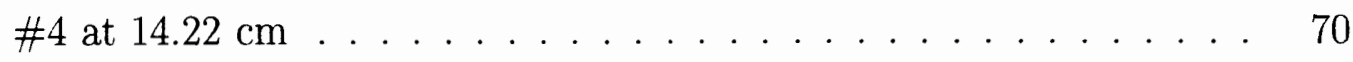

3.25 Magnified Spark-Shadowgraph of Boundary Layer Transition with Grid $\# 4$ at $3.56 \mathrm{~cm} \ldots \ldots \ldots \ldots \ldots \ldots \ldots \ldots$ 
4.1 Basic Single-Plate Interferometric System . . . . . . . . . . . . 76

4.2 Light Path Through Single-Path . . . . . . . . . . . . . . . 78

4.3 Bragg Cell Shutter System . . . . . . . . . . . . . . . . . 80

4.4 Shock Wave Frequency Spectrum . . . . . . . . . . . . . 86

4.5 Representative Pressure Transducer Gain . . . . . . . . . . . . . 88

4.6 Kulite XCQ-062-50 Pressure Transducer . . . . . . . . . . . . . . . . 89

4.7 Pressure Transducer Frequency Response . . . . . . . . . . . . . 90

4.8 Dynamic Response of Pressure Transducer to an Impulse Input . . . 90

4.9 Micro Channel Plate Design $[$ Sturz, 1995] . . . . . . . . . . . 95

4.10 Time Delay Circuit . . . . . . . . . . . . . . . . . . . . 99

4.11 Bridge Circuit . . . . . . . . . . . . . . . . . . . . . . 103

5.1 Rendering of Shock Passage Through Studied Blade Passage . . . . . 107

5.2 Properties Across a Moving Shock Wave . . . . . . . . . . . . . 113

5.3 Hot Wire Corrected Unsteady Density Trace, Hole \#1 . . . . . . . . 118

5.4 Hot Wire Corrected Unsteady Density Trace, Hole \#2 _ . . . . . . . 119

5.5 Hot Wire Corrected Unsteady Density Trace, Hole \#3 . . . . . . . 120

5.6 Hot Wire Corrected Unsteady Density Trace, Hole \#4 . . . . . . . . 121

5.7 Hot Wire Corrected Unsteady Density Trace, Hole \#5 . . . . . . . . 122

5.8 Hot Wire Corrected Unsteady Density Trace, Hole \#6 . . . . . . . 123

5.9 Hot Wire Corrected Unsteady Density Trace, Hole \#7 . . . . . . . . 124

5.10 Hot Wire Corrected Unsteady Density Trace, Hole \#8 . . . . . . . 125

5.11 Hot Wire Corrected Unsteady Density Trace, Hole \#9 . . . . . . . . 126

5.12 Hot Wire Corrected Unsteady Density Trace, Hole \#10 . . . . . . . 127

5.13 Hot Wire Corrected Unsteady Density Trace, Hole \#11 . . . . . . . 128

5.14 Hot Wire Corrected Unsteady Density Trace, Hole \#12 . . . . . . . . 129

5.15 Hot Wire Corrected Unsteady Density Trace, Hole \#13 . . . . . . . 130

5.16 Hot Wire Corrected Unsteady Density Trace, Hole \#14 . . . . . . . . 131

5.17 Hot Wire Corrected Unsteady Density Trace, Hole \#15 . . . . . . . . 132

5.18 Binarized Interferogram of Unheated Flow in Area $1 \ldots \ldots$. . . . 135

5.19 Thinned Interferogram of Unheated Flow in Area $1 \ldots \ldots$ 
6.1 Interferogram of Unheated Flow, Area $1 \ldots \ldots$. . . . . . . . 140

6.2 Interferogram of Unheated Flow, Area 2 . . . . . . . . . . . . . . 141

6.3 Interferogram of Heated Flow, Area 1 . . . . . . . . . . . . . 142

6.4 Interferogram of Heated Flow, Area $2 \ldots \ldots \ldots$

6.5 Unsteady Interferogram in Area 1,178 $\mu s$ Delay . . . . . . . . . . . 144

6.6 Unsteady Interferogram in Area 1, $258 \mu s$ Delay . . . . . . . . . . . 145

6.7 Unsteady Interferogram in Area $1,337 \mu s$ Delay . . . . . . . . . . 146

6.8 Unsteady Interferogram in Area 1,393 $\mu s$ Delay . . . . . . . . . . 147

6.9 Unsteady Interferogram in Area 1, $465 \mu s$ Delay . . . . . . . . . . . . 148

6.10 Unsteady Interferogram in Area 1, $518 \mu s$ Delay . . . . . . . . . . . . 149

6.11 Unsteady Interferogram in Area 2, $178 \mu s$ Delay . . . . . . . . . 150

6.12 Unsteady Interferogram in Area 2, $258 \mu s$ Delay . . . . . . . . . . . 151

6.13 Unsteady Interferogram in Area 2,337 $\mu s$ Delay . . . . . . . . . . 152

6.14 Unsteady Interferogram in Area 2,393 $\mu$ s Delay . . . . . . . . . . . 153

6.15 Unsteady Interferogram in Area 2, $465 \mu s$ Delay . . . . . . . . . . 154

6.16 Unsteady Interferogram in Area 2, $518 \mu s$ Delay . . . . . . . . . . 155

6.17 Relative Positioning of Area 1 and Area 2 to the Studied Blade Passage 156

6.18 Density Field for Adiabatic Flow . . . . . . . . . . . . . . 158

6.19 Density Field for Heated Flow . . . . . . . . . . . . . . . . . . 159

6.20 Density Field for Unsteady Flow, $178 \mu$ s Delay . . . . . . . . . . 160

6.21 Density Field for Unsteady Flow, $258 \mu$ s Delay . . . . . . . . . . . . 162

6.22 Density Field for Unsteady Flow, $337 \mu s$ Delay . . . . . . . . . . . 163

6.23 Density Field for Unsteady Flow, $393 \mu$ s Delay . . . . . . . . . . . . 164

6.24 Density Field for Unsteady Flow, $465 \mu s$ Delay . . . . . . . . . . . . 165

6.25 Density Field for Unsteady Flow, $518 \mu s$ Delay . . . . . . . . . . . 166 


\section{List of Tables}

3.1 Case Specific BL2D Input Flow Conditions . . . . . . . . . . . . 41

3.2 General BL2D Input Conditions . . . . . . . . . . . . . . . . 41

3.3 Turbulence Grid Characteristics . . . . . . . . . . . . . 55

4.1 Optical Component Sources . . . . . . . . . . . . . . . 88

4.2 Pressure Transducer Sources and Critical Properties . . . . . . . . 89

4.3 CCD Camera Sources . . . . . . . . . . . . . . . . . 99

5.1 Time Delay Calibration . . . . . . . . . . . . . . . . . . . . 113 


\section{Nomenclature}

\begin{tabular}{ll}
$c_{f}$ & coefficient of friction \\
$\mathrm{d}$ & diameter \\
$\mathrm{F}$ & velocity \\
$f_{A B_{f}}$ & number of fringes between A and B, with flow \\
$f_{A B_{n}}$ & number of fringes between A and B, no flow \\
$\mathrm{H}$ & temperature \\
$i$ & streamwise direction index \\
$\mathrm{K}$ & constant, see Eq. 3.1 \\
$k$ & normal direction index \\
$\mathrm{L}$ & test section span \\
$L_{x}$ & turbulence length scale \\
$\mathrm{l}$ & reference length \\
$\mathrm{M}$ & Mach number \\
$N_{P r}$ & Prandtl number, laminar \\
$\mathrm{n}$ & index of refraction \\
$\mathrm{P}$ & pressure \\
$\mathrm{p}$ & pressure \\
$\mathrm{Re}$ & Reynolds number \\
$\mathrm{S}$ & surface distance \\
$\mathrm{T}$ & temperature \\
$T_{u}$ & turbulence intensity \\
$\mathrm{t}$ & transverse curvature \\
$\mathrm{U}$ & freestream velocity \\
\hline
\end{tabular}




\section{subscripts}

0

0

normal freestream velocity spanwise freestream velocity instantaneous axial velocity instantaneous normal velocity instantaneous spanwise velocity axial distance downstream distance normal coordinate spanwise coordinate complex wave number complex wave number pressure gradient parameter wedge opening angle eddy viscosity functions ratio of specific heats complex freestream distribution frequency complex phase velocity streamwise velocity complex amplitude function density temperature local blade surface coordinate local transformed normal coordinate streamwise intermittency distribution perturbation of fluctuating mean value 


$\begin{array}{ll}1 & \text { ahead of shock wave } \\ 2 & \text { behind shock wave } \\ \text { c } & \text { corrected } \\ \text { e } & \text { edge, exit } \\ \text { m } & \text { measured } \\ \text { ref } & \text { reference condition } \\ \text { s } & \text { shock }\end{array}$

superscripts

$\begin{array}{ll}\text { n } & \text { current iteration stage } \\ & \text { stationary frame }\end{array}$

acronyms

BC Bridge Compensation

CCD Charge Coupled Device

CFD Computational Fluid Dynamics

CVL Copper Vapor Laser

ESP Electronically Scanned Pressure

FSL Free Shear Layer

FST Free Stream Turbulence

ILPT Isentropic Light Piston Tunnel

LE Leading Edge

MCP MicroChannel Plate

NGV Nozzle Guide Vane

OHR Over Heat Ratio

PSI Pressure Systems Instrument

TE Trailing Edge 


\section{Chapter 1}

\section{Introduction}

The aircraft industry, both commercial and military, is a driving force behind the direction of current turbine engine research. Rapid increases in fuel costs and depleting natural resources have dictated that aircraft gas turbine engines operate at the highest possible efficiency over a larger working range and be produced with lower manufacturing effort and cost. This will require a reduction in size, weight and number of stages from present day turbine engine designs leading to increased aerodynamic stage loading. This demand for greater efficiency and increased aerodynamic stage loading in gas turbine engines, particularly in military aircraft, has recently moved the flow regime of high work turbine stages into the transonic regime. The optimization of the aerodynamic and thermal performance of turbomachinery blading in the highly unsteady environment created by transonic rotor and stator turbine blades is an overriding concern in the turbine industry today [1] [2].

The optimization of the aerodynamic and thermal performance of turbomachinery blading requires a detailed knowledge of the internal flow field with respect to the factors that induce losses and promote heat transfer to the blade surface. These factors include boundary layer growth, boundary layer transition location, trailing edge shock characteristics and mixing losses downstream of the trailing edge. Internal flow fields encountered in current turbomachines are not only viscous and compressible but also highly unsteady. The state of the art in axial turbomachines has advanced to the point where further improvements will have to come from a better understanding of 
unsteady phenomena which occur in turbomachines. It has been widely documented that these flows have a significant influence on the efficiency, reliability, aeroelastic stability, forced response and noise generation in modern axial-flow turbines.

Flow unsteadiness in turbine engines arises largely from the relative motion of the blade rows in the alternately stationary and rotating turbine stages. There are three major sources of flow unsteadiness in the interaction between turbine rotor and stator blade rows. The first cause of flow unsteadiness within a rotor turbine blade stage is a purely subsonic effect termed potential flow interaction [3]. In this form of interaction, the complete inviscid flowfield around each blade is affected by the presence of the neighboring upstream and downstream blade rows.

The second cause of flow unsteadiness in rotating turbine stages is wake passing. The wake passing effect is due to the repeated passage of a downstream blade row through the wakes shed from the trailing edges of the upstream stationary blade row. Of the three major sources of flow unsteadiness, potential flow interaction and wake effects have received considerable prior attention.

Third, for transonic turbines, significant unsteadiness occurs due to the nozzle guide vane (NGV) trailing edge shock structure impinging on the downstream rotor blades, Fig. 1.1 [4]. This source of flow unsteadiness, shock-wave passing, has received much less attention due to the complexity of creating repeatable passing shock events at the required spacing to simulate a real engine environment. However, if progress is to be made in modern turbine blade design, reliable experimental results as well as computational methods will be required in this flow regime.

Four types of experimental facilities are in general use to simulate and investigate the unsteady aerodynamic aspects of axial-flow turbomachines - linear cascades, stationary annular cascades, low speed rotating rigs and high speed rotating rigs. Most experimental studies on unsteady effects in turbines have been conducted in linear cascades. Linear cascades use a finite number of two-dimensional airfoils, representing particular spanwise sections of the turbine blades, to study the subsonic, transonic and supersonic flow phenomena in three-dimensional rotating turbine engines. Linear cascades are widely used, because they provide the most convenient and cost effective way to study a variety of configurations and blade profiles. Also, cascades allow for 


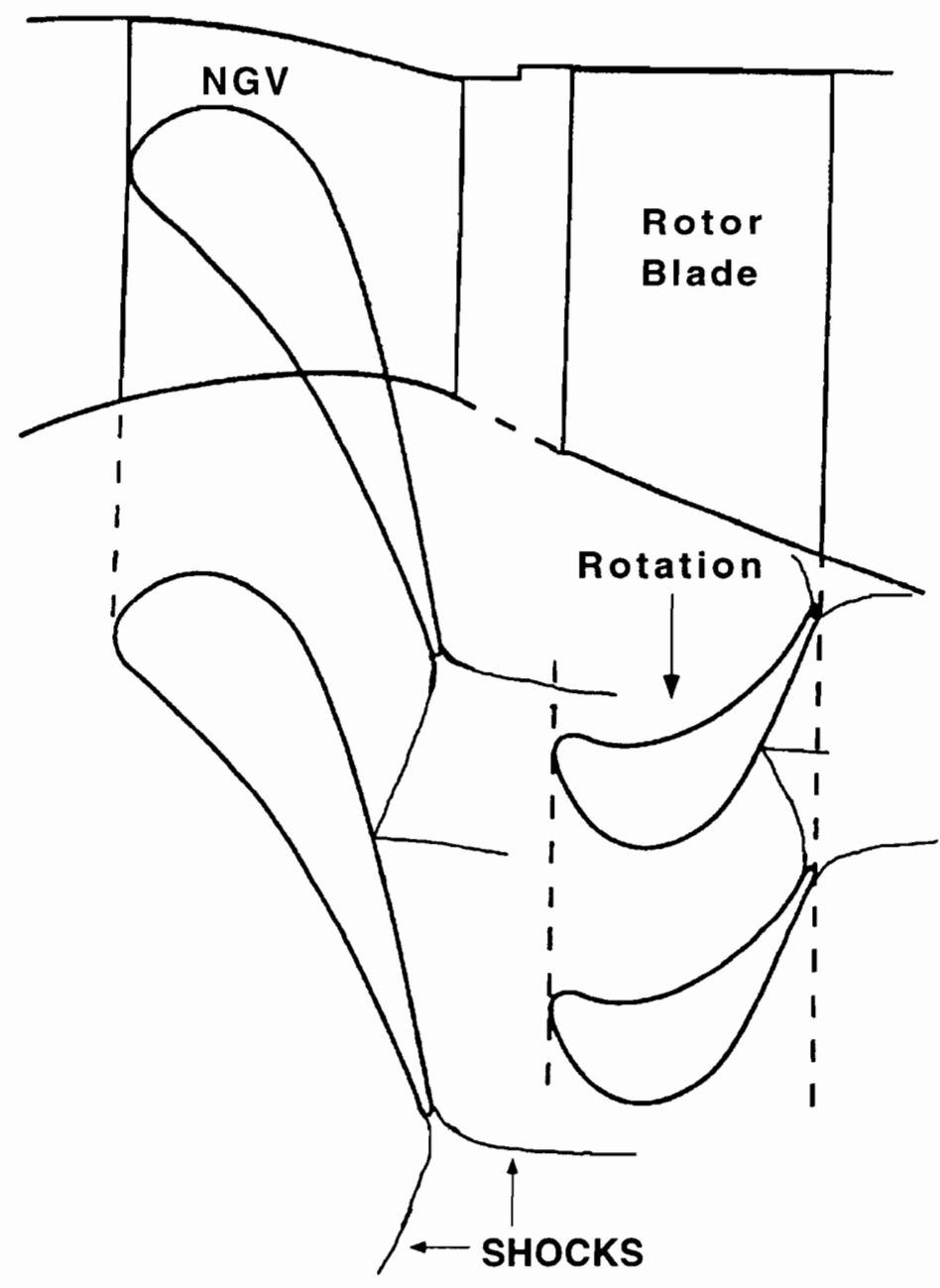

Figure 1.1: Unsteady Shock Wave Passing 
easy flow visualization access and can accommodate large blade dimensions required for detailed measurements. Unfortunately, it is difficult to achieve flow periodicity within a linear cascade [5].

Up until the mid-sixties, blading design relied largely on empirical correlation where component design refinements proceeded mainly from systematic testing and flow calculations carried out, for the most part, with simple analytical methods and linearized theories. However, around 1965, computers came into general engineering use, changing the way experimental research in turbine blading design is both viewed and used. Now, testing is regarded not only as a means of verifying the performance of a given design, but also to calibrate complex computational methods used in the design process. With the introduction of unsteady codes, two challenging problems arise. First of all, validation of these codes must be achieved on a number of realistic configurations to build confidence in the computed results among designers. This requires a large database of reliable unsteady data for useful comparisons with computed results. Secondly, questions arise concerning how designs will be compared within this unsteady environment. Will steady state averages be adequate, or will time resolved comparisons need to be made? If steady state data is adequate, what comparisons will be relevant $[6]$ ?

Recently, the transonic turbine cascade facility at Virginia Tech has been modified to allow the study of unsteady passing shock flow. Here, a shock tube is used to pass a two-dimensional shock along the cascade leading edge in order to simulate the shock impingement from a nozzle guide vane row onto the first high pressure rotor stage in a transonic turbine engine. The specifics of this modification will be discussed in Sec. 2.3. Therefore, it was desirable to alter the previous steady interferometric flow visualization system developed by T. Kiss [7] to allow for the quantitative study of this phenomena. It was the purpose of this investigation to develop an experimental system capable of measuring the time history of the density field in the blade passage of a transonic cascade during an unsteady shock passing event. These measurements will be used in the future to fine-tune unsteady computational fluid dynamic (CFD) calculations to be made of the unsteady flowfield within this cascade. Also, an attempt will be made to understand the large variations in lift and angle of incidence found 
by other researchers.

Blading design methods often use the profile velocity distribution as a criterion to evaluate the aerodynamic quality of a given design, since there is a very close connection between profile losses caused by the state of the blade boundary layer and the blade surface velocity distribution. Also, as turbine entry temperatures move higher in an attempt to increase thrust and lower specific fuel consumption, accurate prediction of the blade heat transfer rate is critical. As the thermal insulating properties of the boundary layer vary greatly depending on its state (laminar, transitional or turbulent) it is necessary to determine and possibly control the boundary layer state if accurate experimental heat transfer investigations are to be made. This led us to conduct a coordinated experimental/computational study of transition in our turbine cascade.

\subsection{Literature Review}

To date, the most extensive research conducted on unsteady wake and shock passing was performed in the Isentropic Light Piston Tunnel (ILPT) of Oxford University in Oxford, England. In this facility, passing wakes and shocks are generated in a stationary cascade of highly-loaded transonic turbine rotor blades by moving a row of wake generating rods past the cascade at the required rotor velocity. Prior experiments had shown that, at the rotor inlet position, the NGV wakes were symmetrical and could be simulated by the wakes of small circular cylinders. It needs to be noted that ideally the wake and shock fronts should be perfectly two-dimensional. This ideal could only be achieved by passing a rectilinear "ladder" of bars past the stationary cascade. However, this type of design is not mechanically feasible. Therefore, the Oxford tunnel approximates ideal two-dimensional shocks and wakes over a single cascade passage by mounting the bars on the rim of a large-diameter rotating disk. This design also differs from the "Squirrel cage" apparatus used by Pfeil et al. [8] and Bayley and Priddy [9]. Flexibility in this design allows the effects of wake size, spacing, and combinations of wake and shock passing as well as free stream turbulence to be assessed. Periodic shocks and wakes were produced by fitting the disk 
with a large number of bars, while only two bars on the disk gave isolated shock/wake events. Each rotating bar, constructed of stranded wire, generates a bow shock wave, a wake and a recompression shock wave. This differs from the flow produced in a NGV, which is absent the bow shock wave. This apparatus was first introduced in a paper by Doorly and Oldfield [10], where schlieren studies were made of the unsteady effects of wake passing both with and without shock waves. These schlieren results were then used to determine both the external flow history and to compare with simple numerical predictions of the expected wake trajectory through the blade passage.

Later that year, using the same facility, Doorly and Oldfield reported on the effects of passing shock waves and wakes on the heat transfer [3]. The ILPT was run at a free stream total temperature of $432 \mathrm{~K}$, corresponding to a gas-to-wall temperature ratio of 1.5 and a Reynolds Number based on the blade chord of $2.02 \times 10^{6}$. The rotating bars were moved at a relative Mach number of 0.94 in the 0.32 Mach number cascade inlet flow. The rotating disk was used in the two bar configuration described above to allow complete relaxation of the boundary layer between the shock/wake events. The authors found that the impingement of an isolated shock wave on a naturally laminar suction-surface boundary layer resulted in the formation of what was apparently a separation bubble that quickly collapsed. At the collapse of this separation bubble, a turbulent boundary layer patch appeared which was swept down the blade suction surface, producing a transient spike in the blade surface heat transfer rate. The wake was found to have a similar effect on the boundary layer, producing a characteristic "square wave" type of transition from laminar to a quasi-steady turbulent state and back to laminar. The authors also found that the direct effect of the shock impingement on the heat transfer rate was almost unnoticeably small, though there was a more noticeable effect for the tripped boundary layer. Lastly, high frequency shock wave impingements were found to have a complicated interaction and seemed to indicate that even relatively weak shock waves could have a noticeable effect on the boundary layer transition process.

These results were further supported by Schultz, et. al. [11] in a 1986 paper presented at the 68th(B) AGARD Specialists' Meeting. Detailed blade surface heat 
transfer rate measurements were made with 22 high-response, thin-film surface resistance thermometers, enabling the wake and shock phenomena to be separated. The ILPT was run at a free stream total temperature of $432 \mathrm{~K}$, with an isentropic exit Mach number of 1.18 and a Reynolds number based on the true chord of $0.919 \mathrm{x}$ $10^{6}$. Various grids were used to produce free-stream turbulence levels (FST) of $0.8 \%$ (no grid case) up to $3 \%$. The authors first discussed the effect of the varying inlet incidence angle on the heat transfer rate and surface pressure distribution. It was found that the passage of the reduced velocity wake through the rotor blading gave rise to a time varying change in incidence angle. This variation in incidence angle particularly affected the suction surface heat transfer rate and pressure distribution. A longer region of laminar heat transfer was found with a decreased incidence angle of $-10^{\circ}$ from the design incidence of $58.06^{\circ}$. As was found on the blade profile used by Doorly and Oldfield [3] that the low FST case remained laminar throughout the entire measuring range of the surface heat transfer gages. However, the high FST case became increasingly dominated by sharp transient events as the FST was raised. It was also noted by the authors that the state of the turbine boundary layer was only affected by the transient shock/wake passing events while they were passing through the passage. The heat transfer levels were essentially unchanged far removed in time from the disturbance.

In 1989, Ashworth, et.al., [12] began detailed investigations into the effects of varying FST on the turbulent intermittency factor over the suction surface of a turbine blade. Operating the ILPT with the same blade profile and at the same conditions as Schultz, et. al., 17 thin film gages spaced around the blade suction surface were employed to track heat transfer levels and follow turbulent spot development. Various levels of FST were obtained, from a no grid low of $0.8 \%$ to a high of $3.0 \%$, using a grid of $2 \mathrm{~mm}$ diameter bars placed $208 \mathrm{~mm}$ upstream of the cascade inlet plane. Some of the results discussed by the authors were for the low FST, steady case where the boundary layer remained laminar back to the upstream blade trailing edge shock impingement. However, for the $3 \%$ FST case, the heat transfer rate began to increase, beginning at the crown of the blade $(\mathrm{x} / \mathrm{s}=0.15 \%)$, due to turbulent spot development with an increasing intermittency factor as one travels back along the blade suction 
surface toward the trailing edge. The unsteady case results were also found to be similar to those discussed by Doorly, et. al. Using these results, a prediction code for the turbulent intermittency factor was developed from the method described by Patankar and Spalding [13], incorporating a model for mixing FST into the boundary layer. Allowances were also made for curvature effects and changing free-stream conditions [14].

In 1990, a paper by Johnson and Oldfield, et. al. [15] proposed a different explanation than the separation bubble proposed by Doorly, et. al. [3] for the transient spikes in surface heat transfer rate during the passing shock/wake event. When schlieren images and surface heat transfer measurements clearly detected the presence of a convecting "bubble" moving along the pressure surface, as opposed to the suction surface as seen in previous experimental results, the authors proposed a different explanation. They suggested that the feature was a "vortical bubble". As the shock wave moves past the leading edge of the blade, the incident angle between it and the blade surface increases rapidly. When this occurs, the shock wave mutates from being an oblique wave with a reflection, to a normal wave without a reflection. As the reflected wave continues to propagate away from the surface, a bifurcation is formed. When this bifurcation then moves away from the surface, a Mach reflection with a vortex sheet behind it is created, which then rolls up to form a transient vortex at the blade leading edge (LE). The authors explain that the tests conducted by Doorly and Oldfield [3] were on a different blade profile. The phenomena, then thought to be a separation bubble propagating along the suction surface of the blade, could then be clearly seen as a vortical bubble, formed on the suction surface side of the leading edge. This was encouraging, since careful control of the LE geometry could force the vortical bubble onto the blade surface (suction or pressure) where the thermal loading was less critical.

All of the experimental work described above employed bars moving at high velocities along the leading edge of a linear cascade to produce combined passing shocks and wakes. Using this combination, it is difficult to separate the effects due solely to the passing shocks, because of the non-linear interaction between the wake/shock events. Work recently conducted at Virginia Tech has concentrated exclusively on 
the unsteady effects produced by shock waves passing through transonic turbine rotor cascades to permit the study of the complex processes separately.

$\mathrm{J}$. Collie was the first to attempt developing a method to produce passing shock waves without wakes [16]. Utilizing the explosion from a blank shotgun shell, the blastwave produced is shaped and directed through the cascade test section, parallel to the leading edge of the blade row. This method of shock generation was somewhat limited, since only single shocks could be produced. However, using a specially designed shock shaper, highly repeatable single shocks could be produced, allowing data from numerous single shock events to be coupled. The author used triggered spark shadowgraphs to successfully track the propagation of the shock wave through the studied blade passage. The formation of the vortical bubble described by Johnson and Oldfield was also documented in the shadowgraphs. Other measurements described by the authors centered around significant variations in the blade performance as the shockwave passed through the studied passage. Using surface mounted Kulite XCQ-065 pressure transducers, a 120\% peak-to-peak variation in estimated blade lift was found. Also, wake total pressure traverses found that the cascade loss coefficients fluctuated as much as $40 \%$ near the blade passage center due to shock passing effects.

Recently, the shotgun method employed in the Virginia Tech transonic wind tunnel has been reworked. R. Doughty [17] abandoned the shotgun in favor of a shocktube. With this method, up to three variably spaced shocks can be directed into the cascade test section. Using this design, Doughty repeated the work conducted by Collie for three shockwaves. This system for simulating the unsteady effects of shock waves is what is currently being used in the Virginia Tech Transonic Cascade Wind Tunnel. 


\section{Chapter 2}

\section{Transonic Tunnel Design}

All of the experiments described in this dissertation were conducted in the Virginia Polytechnic Institute and State University Transonic Cascade Wind Tunnel (see Fig. 2.1). This facility is a blow-down type cascade tunnel, providing up to 40 seconds of usable run time. A four-stage Ingersoll-Rand type $\mathrm{H}$ reciprocating compressor, in-line with a heat exchanger, provides the air-supply for the tunnel. From the compressor, the air is diverted through an activated-alumina drying unit which removes water vapor and other contaminants from the air. The air is then stored in two reservoir tanks; here the air pressure is monitored to obtain a pressure of 130 to 140 psi. Upon activation of the tunnel control system, the air flows from the two reservoir tanks through a safety valve and a control valve network. This control valve works on a simple feedback system where a control algorithm attempts to maintain a constant total inlet pressure of 32 psia within the test section. A C program on an IBM $\mathrm{PC}$ provides the actuation of the valve based on feedback received from a pressure transducer.

Both the safety and control valves are pneumatically operated. The control valve is supplied by air from both the shop system and a compressed air-bottle, set at 21 psi. The safety valve has an automatic system that shuts the valve if a gage pressure of $207 \mathrm{kPa}$ (30 psig) is attained. This ensures that no structural damage is incurred by the test section. The flow coming from the valve passes through a $90^{\circ}$ bend, followed by a flow straightener (heat exchanger) consisting of an array of approximately 700 


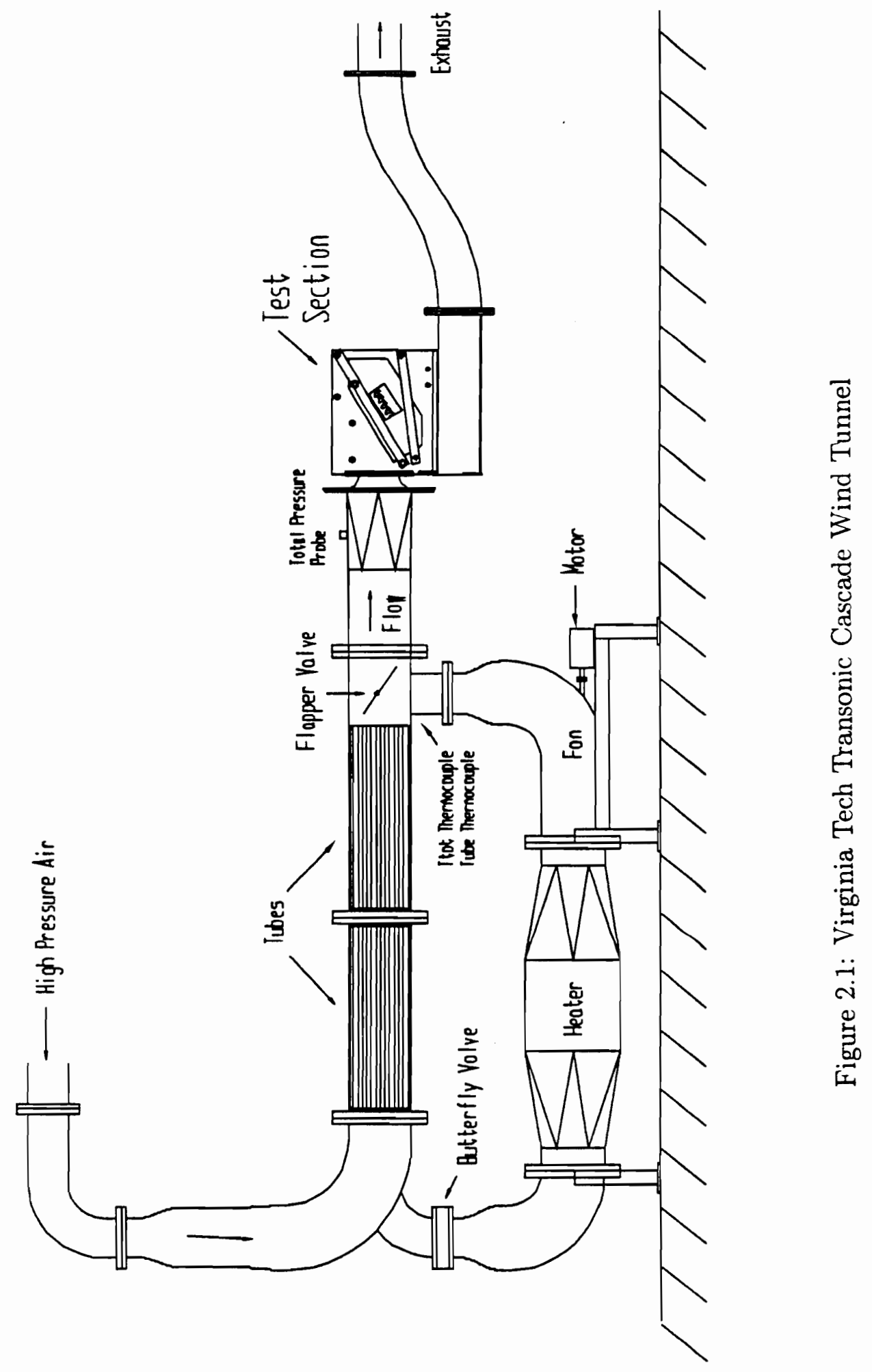


copper tubes before entering the cascade. Upon exiting the flow straighteners, the air passes through a circular to rectangular transition piece and enters the test section. After each tunnel run, it takes 5 to 10 minutes to recharge the tanks to the required pressure.

The flow enters the test section at a Mach number of 0.36 . As the flow passes through the cascade, the flow is choked in the throat formed by the blades and obtains supersonic speeds. After being turned by the blades, the flow is equalized through classic trailing edge "fishtail" shocks.

The exit Mach numbers in turbine cascades are typically described by an isentropic Mach number. This number describes the Mach number at a given location if the flow were isentropic. Thus, if the instantaneous upstream total pressure is given as $\mathrm{p}_{u t}$, than the instantaneous isentropic exit Mach number, $\mathrm{M}_{e_{i}}$, is

$$
M_{e_{i}}=\sqrt{\frac{2}{\gamma-1}\left(\left(\frac{p_{t u_{i}}}{p_{e_{i}}}\right)^{\frac{\gamma-1}{\gamma}}-1\right)}
$$

where $\mathrm{p}_{e_{i}}$, is the instantaneous, spatially averaged exit static pressure. The instantaneous isentropic exit Mach number is changing throughout the run because of imperfect control of the upstream total pressure by the feedback managed pneumatic control valve. Therefore, time averaging of the instantaneous isentropic exit Mach number is desired. Using the procedure outlined by Kiss [7], the time averaged isentropic Mach number can be written as

$$
M_{e}=\frac{1}{328} \sum_{i=i_{1}}^{i_{1}+327} M_{e_{i}}+\frac{1}{328} \sum_{i=i_{2}}^{i_{2}+328} M_{e_{i}} .
$$

The design time averaged isentropic exit Mach number for this cascade was 1.26. All cascade tests described in this dissertation were run at this exit Mach number.

\subsection{Test Section and Blade Design}

The cascade consists of eleven turbine blades, two end blocks and either two Plexiglass or aluminum endwalls, used for general aerodynamic measurements or interferometry, 
respectively, Fig. 2.2. Specifics of the construction of the aluminum endwalls will be discussed at length in Section 2.2. The blades are secured to the endwalls by a pin and countersunk allen screw combination, and the endblocks are secured to the endwalls with countersunk allen screws. The cascade is secured in the test section by grooved slots located in the aluminum doors. The doors are secured to the test section by two steel bars locked in place with two $3.175 \mathrm{~cm}\left(1 \frac{1}{4}^{\prime \prime}\right)$ nuts and two $1.905 \mathrm{~cm}\left(\frac{3}{4}^{\prime \prime}\right)$ nuts. Both the doors and the cascade are equipped with O-ring seals to reduce air leakage from the cascade. The Plexiglass endwalls are transparent to allow optical measurements such as shadowgraphs or oil flow visualization.

The cross section and dimensions of the blade studies are shown in Figure 2.3. The blade was designed by General Electric. The blade has a $3.81 \mathrm{~cm}$ (1.5 in) axial chord a $4.5 \mathrm{~cm}$ (1.77 in) aerodynamic chord and a $5.08 \mathrm{~cm}$ (2.0 in) aerodynamic chord with an approximate turning angle of $127^{\circ}$. The span is $5.08 \mathrm{~cm}(2.0 \mathrm{in})$ and the blades are spaced a distance of $3.81 \mathrm{~cm}$ (1.50 in) apart. Labeling the 11 blades from the top of the cascade to the bottom (right to left), the blades of interest in this study are blades \#6 and \#7, Fig. 2.4 .

\subsubsection{Cascade Flow Characteristics}

While using a stationary cascade has many advantages, mechanical and otherwise, over rotating blades, obtaining flow periodicity in a linear turbine cascade is difficult. This is because the trailing edge shock waves of the cascade row are reflected back into the cascade exit flow by the free shear layer. The free shear layer (FSL) is created by the discharge of the supersonic jet into the ambient air behind the cascade blade row. The shock wave impingement and subsequent reflection can produce strong and spurious flow disturbances [18]. Depending upon blade shape, flow angle and Mach number, the reflected shocks and expansions will vary in strength and effect. Many researchers have found that the most effective method of achieving acceptable flow periodicity within a transonic turbine cascade is to have a high $(>6-7)$ number of blades [18] [19]. For that reason, this cascade design uses a row of 11 blades. Oil flow visualization and spark-shadowgraph studies have been conducted to ensure adequate 


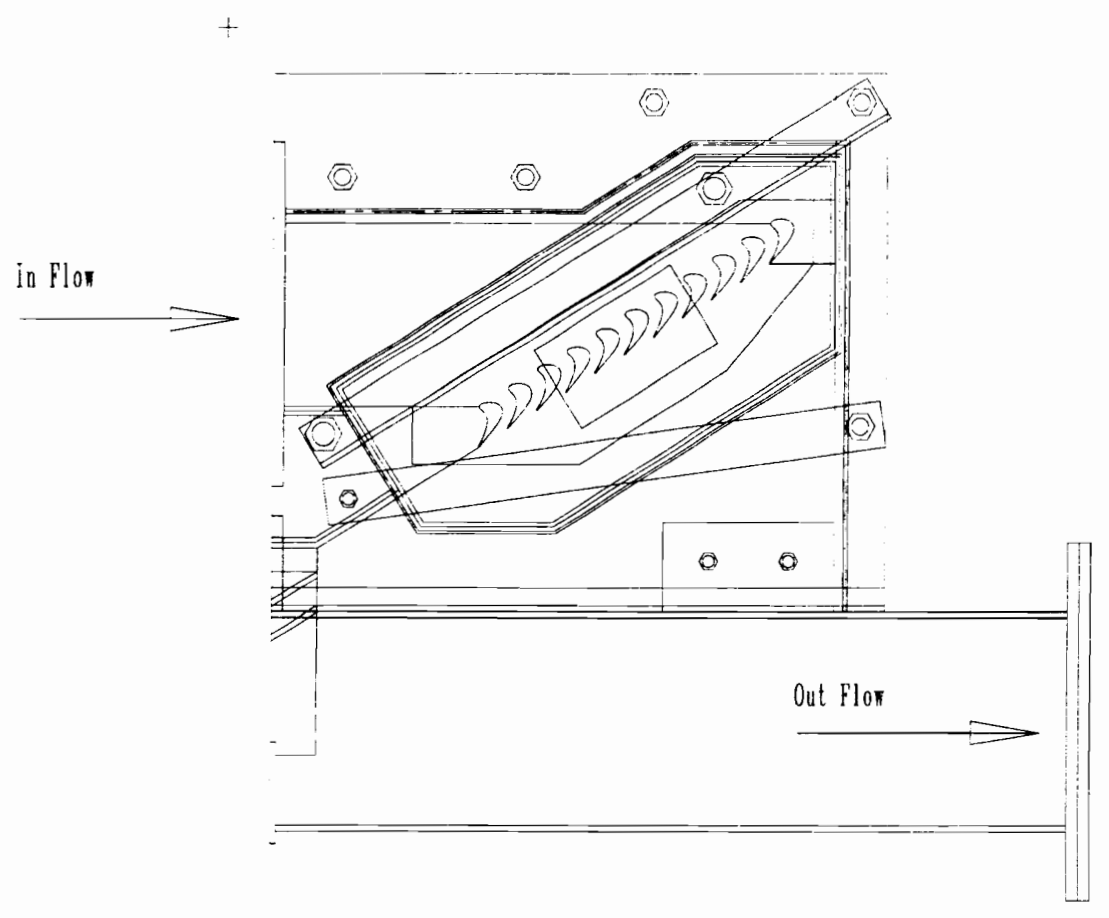

Figure 2.2: Virginia Tech Transonic Cascade Test Section 


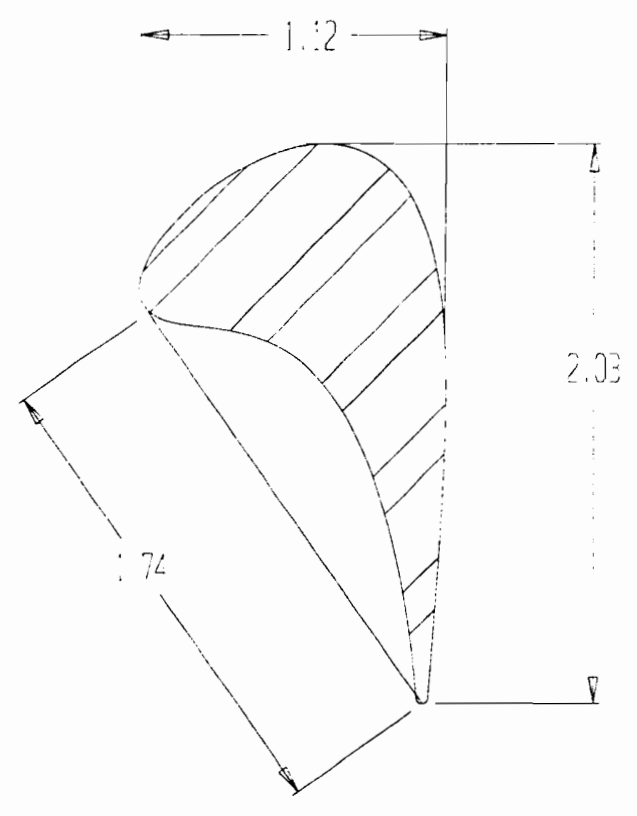

Figure 2.3: Transonic Turbine Blade Profile 


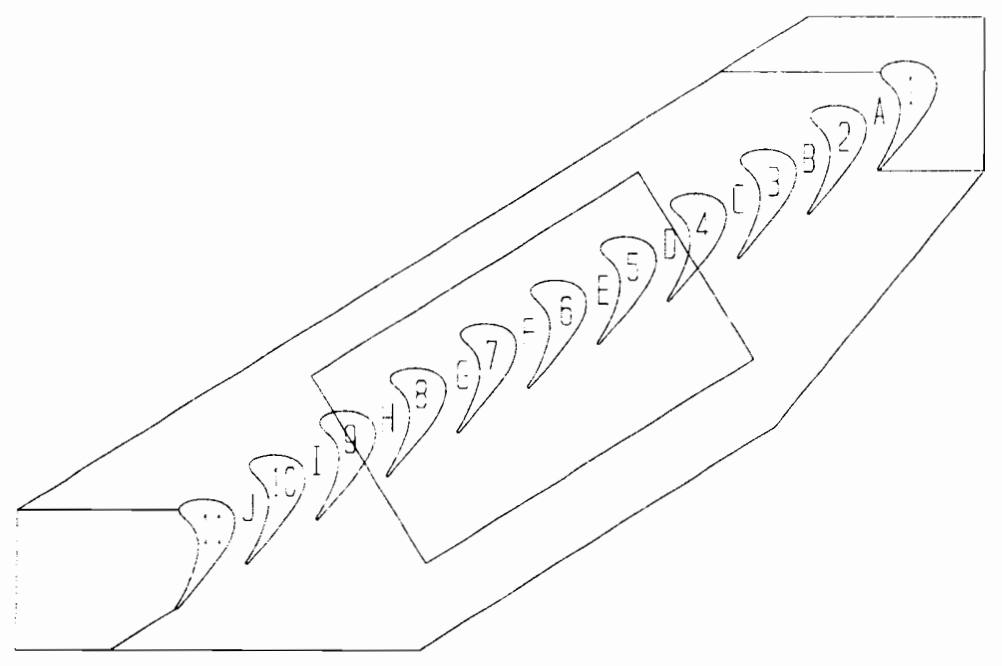

Figure 2.4: Transonic Turbine Cascade 
flow periodicity. Figure 2.5 is a spark-shadowgraph of the overall flowfield through the central blade passages and Fig. 2.6 is an oil flow visualization of the cascade endwall. As is apparent from these photographs, the flow in this cascade exhibits good periodicity.

Another concern in linear turbine cascade design is the two-dimensionality of the flow. It has been shown that in a turbine cascade the approaching side-wall boundary layer will roll up into a "horseshoe" vortex around the blade leading edge. One branch of the vortex moves along the suction surface of the blade while the other branch moves across the passage toward the suction surface of the lower blade in the cascade row [18]. This is a major factor affecting the two-dimensionality of the cascade flow. It is important to show that the cascade flow is two-dimensional at the test section centerline, where all experimental measurements are to be taken. Both an upstream endwall boundary layer survey and blade surface oil flow visualization was performed to investigate the dimensionality of the flow in this cascade. The boundary layer survey found that the boundary layer thickness was less than $0.635 \mathrm{~cm}\left(\frac{1}{4}\right.$ in $)$ thick on each endwall, leaving over $3.81 \mathrm{~cm}\left(1 \frac{1}{2} \mathrm{in}\right)$ of core flow through the $5.08 \mathrm{~cm}$ ( 2 in) span cascade. The fact that the centerline flow is generally two-dimensional is also supported by the blade surface oil flow pictures, Fig. 2.7.

Since it is not possible to take many of the required flow measurements in the tunnel simultaneously, the flowfield repeatability is an important concern. Because the flow in the tunnel is choked, the average isentropic exit Mach number is a good monitor of the state of the tunnel flowfield. It was found that the isentropic exit Mach number, as controlled by setting the upstream total pressure, was repeatable to within $\pm 0.01[20]$.

\subsubsection{Blade Static Pressure Measurements}

A survey of the blade surface static pressure distribution was performed to ensure the fabricated blade static pressure distribution matched the distribution calculated by General Electric. To facilitate this, surface pressure taps were fabricated in the blade 


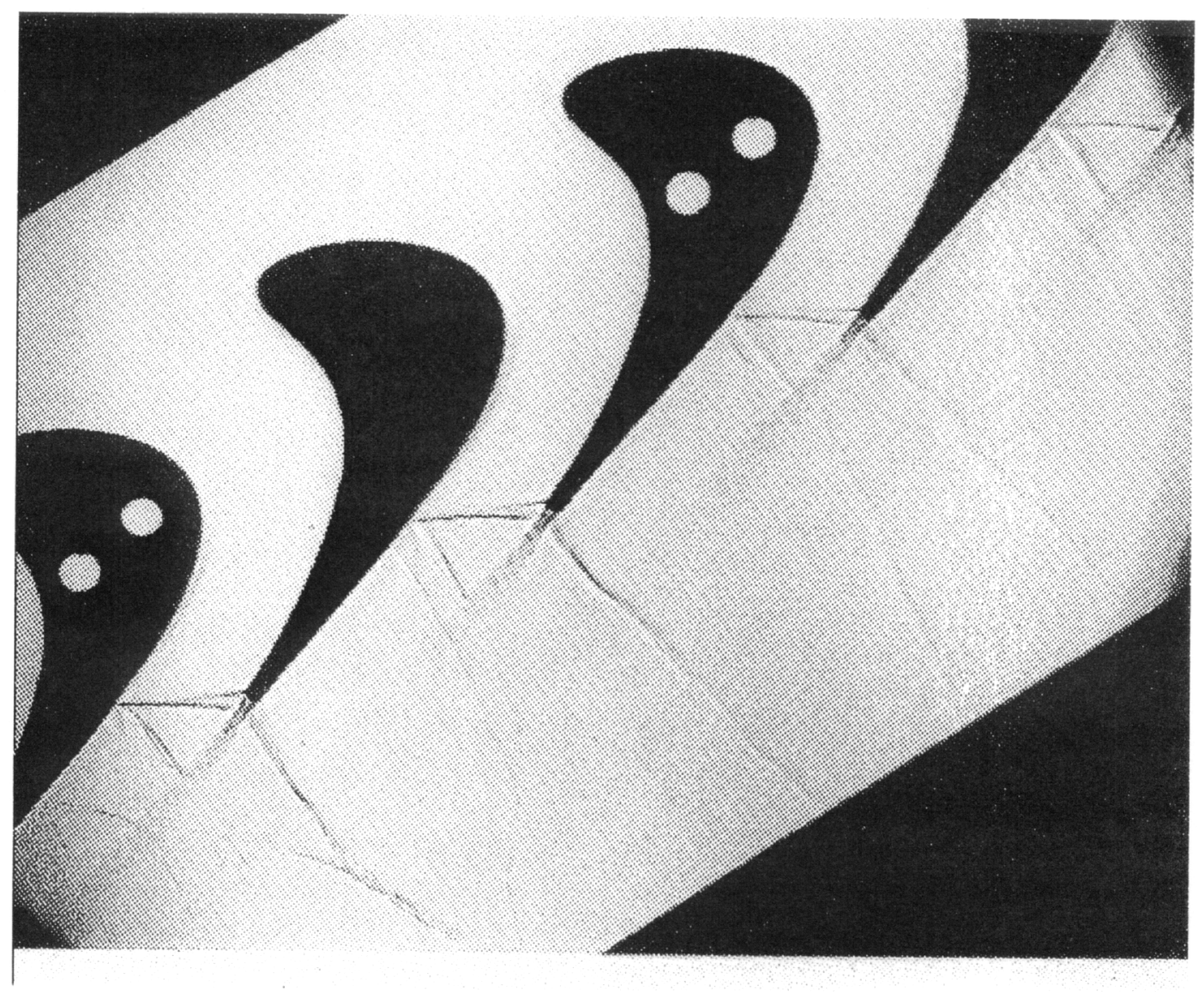

Figure 2.5: Transonic Turbine Cascade Shadowgraph 


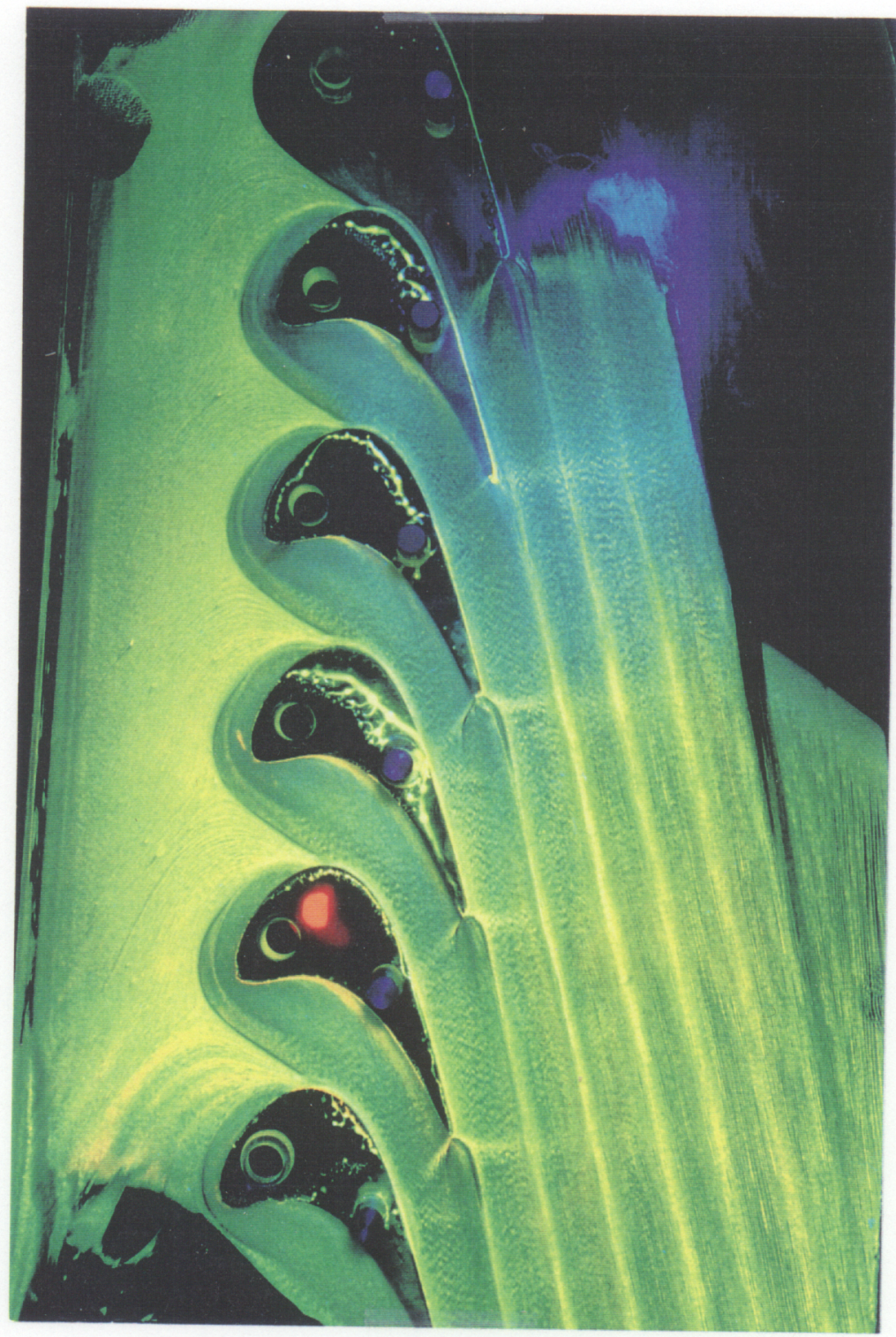

Figure 2.6: Oil Flow Visualizatiion of Cascade Endwall 


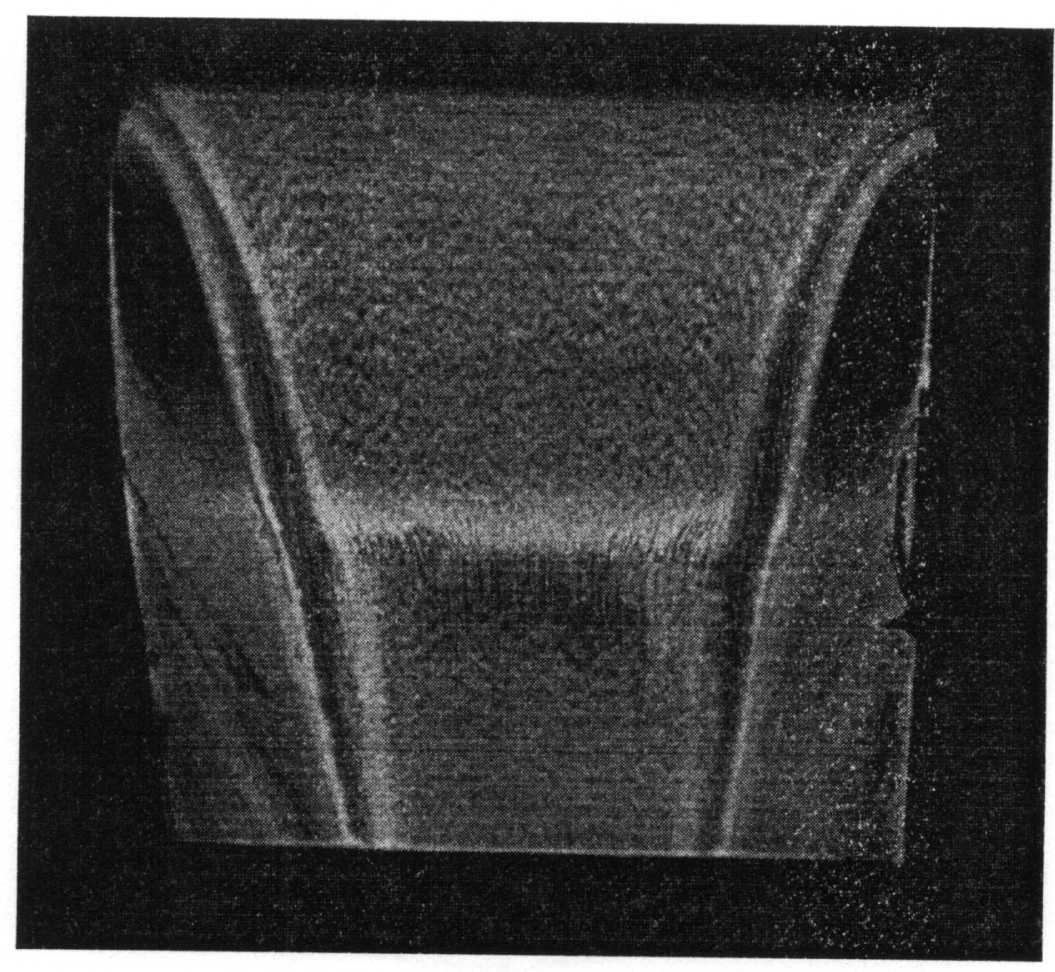

Figure 2.7: Oil Flow Visualization of Single Blade Suction Surface 
surface by the Aerospace and Ocean Engineering Shop at Virginia Tech. The positioning of the tap locations depended mainly on construction considerations including blade size and pinhole, cooling hole and screw hole locations within the blade.

The static pressures were measured using a Pressure Systems Incorporated (PSI) Model 780B Electronically Scanned Pressure (ESP) sensors and a microcomputer based data acquisition system. A system calibration is carried out before each run against a Diaquartz 215 DS transducer, producing an accuracy of $0.0035 \%$ full scale psi. The measurement system can achieve high data rates for multi-pressure measurement applications, since it has a single transducer per port. The ESP sensor that is used has 32 pressure inputs and a range of \pm 20 psig. The PSI system can measure pressures up to a rate of 20,000 measurements per second.

For measuring the blade surface static pressure distribution, it was important to get an average value over the portion of the run where all experimental data would generally be collected. Therefore, a total collection time of 10 seconds, triggered manually after the tunnel control reached a steady value, was chosen. During the 10 second window, 5 sets of data (evenly spaced over the 10 second window) each with a duration of 1 second were taken. Twenty data frames were averaged per measurement set. This means that a total of 100 data points were collected at each tap location over the 10 second interval. The raw gage pressure data was converted to an isentropic Mach number using Eq. 2.1. Three separate runs were made at the design exit Mach number. These runs were then averaged to produce the final computed isentropic Mach number.

Figure 2.8 shows the good comparison of the experimental isentropic Mach numbers and the values computed by General Electric.

\subsection{Optical Window Construction}

In order to provide the needed optical access to the tunnel, it was necessary to construct irregularly shaped windows to fit only in the studied blade passage. A previous attempt at constructing solid glass windows failed due to the large stresses caused by the blade support pins. Therefore, it was decided that glass window inserts which 


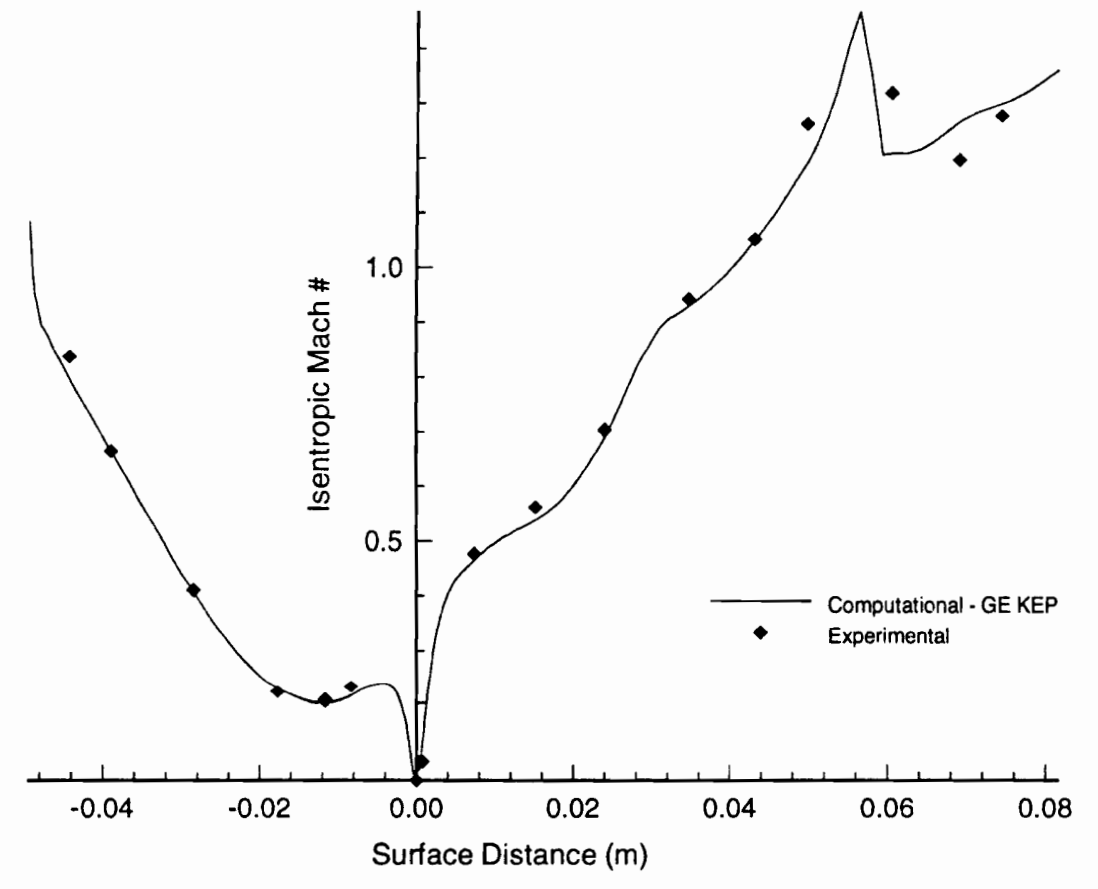

Figure 2.8: Isentropic Mach Number Distribution on Blade Surface 
could be placed within a single blade passage would be constructed. Because of the small physical dimensions of the blade passage, it was necessary to slightly modify the blade pin arrangement. Also, even with the modified pin arrangement, it was still be necessary for the glass to be cut in an intricate shape. Using $\frac{\lambda}{4}$ flatness glass, an optical company which specialized in glass cutting, manufactured the glass window inserts. Also, it was decided that in order to strengthen the modified pin holes and to avoid some reoccurring problems with the Plexiglas, the endwalls were to be constructed of aluminum. Therefore, the CNC machine in the Virginia Tech Mechanical Engineering Department was used to machine the window seats and the aluminum endwalls.

One major advantage of the glass inserts over the Plexiglas is that now it is possible to take schlieren photographs, as opposed to the simple spark-shadowgraph.

\subsection{Shock Wave Generation}

The shock wave generation method developed for use in the transonic turbine cascade is a variation on the shock tube system of Merritt and Aronson [21]. Utilizing a pressure-burst diaphragm shock tube, up to three shock waves are shaped and directed into the top of the transonic test section parallel to the leading edge line of the blades. The shock tube is constructed of extra heavy duty (Schedule 120) steel pipe of $76 \mathrm{~mm}$ nominal diameter with standard 600 pound flanges with lead gaskets (See Figs. 2.9, 2.10 and 2.11). The driver section is 5 meters in length, while the driven section is 10 meters in length. The cap is a standard 600 pound flange with three $12.7 \mathrm{~mm}\left(\frac{1}{2}\right.$ in $)$ NPT holes, arranged in an equilateral pattern. Three pressure rated conduits extend from the end cap of the shock tube up to the top of the test section to create the three separate shocks. The length of each tube can be varied to delay the entrance of the second and third shock into the test section. A nominal spacing of $200 \mu \mathrm{s}$ is used to simulate actual conditions in a turbine engine. Mylar sheets, cut into circles, are used as the diaphragm material. The diaphragm was burst by increasing the driver pressure (here supplied by helium to increase the shock pressure ratio) until the diaphragm failed [17]. For the diaphragm area used here, a pressure of 24 psig per 
mil thickness is required to induce diaphragm failure. A thickness of 24 mils was used for all of the shock tests discussed in this dissertation, requiring a driver pressure of 576 psig for diaphragm failure.

To produce three traveling shocks, three separate ducts, leading from the shock tube to the test section, were required. In order to minimize wave distortion and produce clean shocks, free of excessive or strong reflections, flexible pressure rated conduits were chosen to transmit the shocks. It has been shown [22] that bends with large radii of curvature produce the best stability in a transmitted shock and the flexible duct could easily be manipulated to produce turns with large radii of curvature. Lastly, a shock shaper has been designed to reduce reflected shocks and create a two-dimensional shock face at the studied blade passage. Therefore, it is necessary for the shaper to convert the circular planar shock (in the duct) into a rectangular planar shock front, while minimizing shock attenuation, reflections and other distortions. The final shaper designed by Doughty was tapered with a divergence angle of 24 degrees. Shadowgraph studies show that the shock is, in fact, fairly twodimensional at the blade passage being studied [23]. Also, reflected shocks, created as each shock wave enters the test section, are shown to dissipate before reaching the blade passage of interest. Figure 2.11 illustrates the positioning of the shock shaper relative to the cascade and shows a representative shock positioned within the test section. Shadowgraph and static pressure studies have also shown the shock locations and strengths to be repeatable to within $\pm 10 \mathrm{~ns}$ and $\pm 1.0 \mathrm{psi}$, respectively [17].

\subsection{Heated Flow}

The cascade tunnel also has the capability to produce heated flow conditions to simulate the heat transfer conditions in an actual engine environment. A heating loop, consisting of two bundles of heat exchanger tubes, an electric heater and an axial flow fan, allow flow temperatures of over $80^{\circ} \mathrm{C}$ to be achieved within the cascade test section as shown in Fig. 2.1.

In between runs a flapper valve, located directly upstream of the test section nozzle, is closed and a second valve, located upstream of the flow straightener, is 

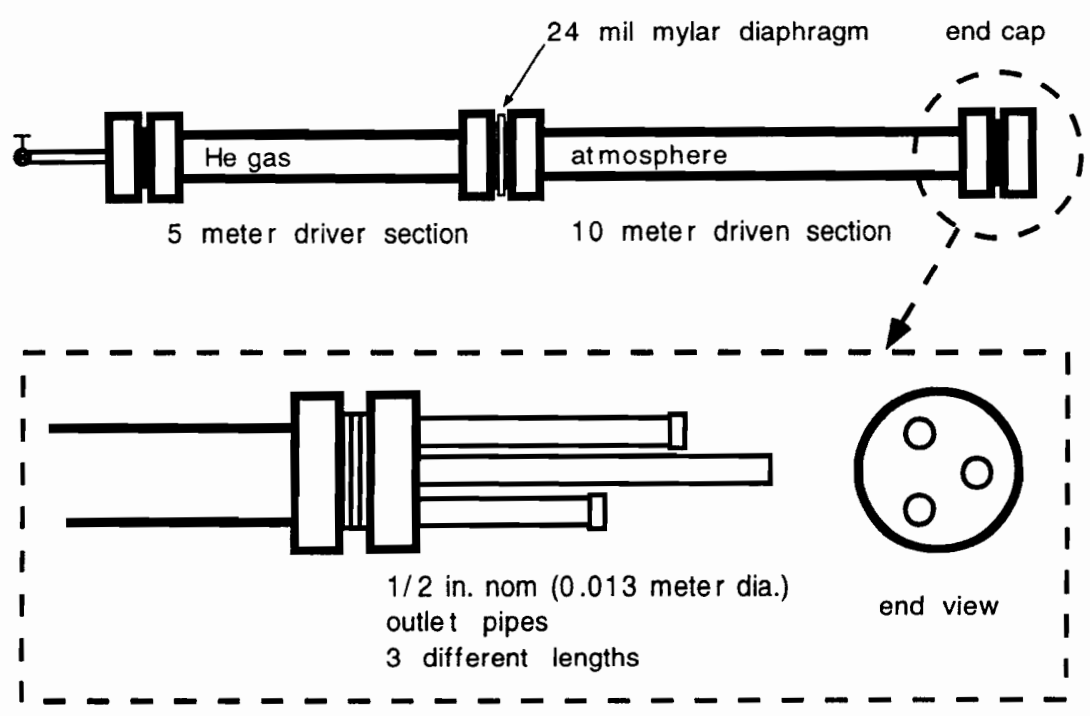

Figure 2.9: Shock-Tube Construction [Doughty,1994] 
Sudden Expansion Shaper

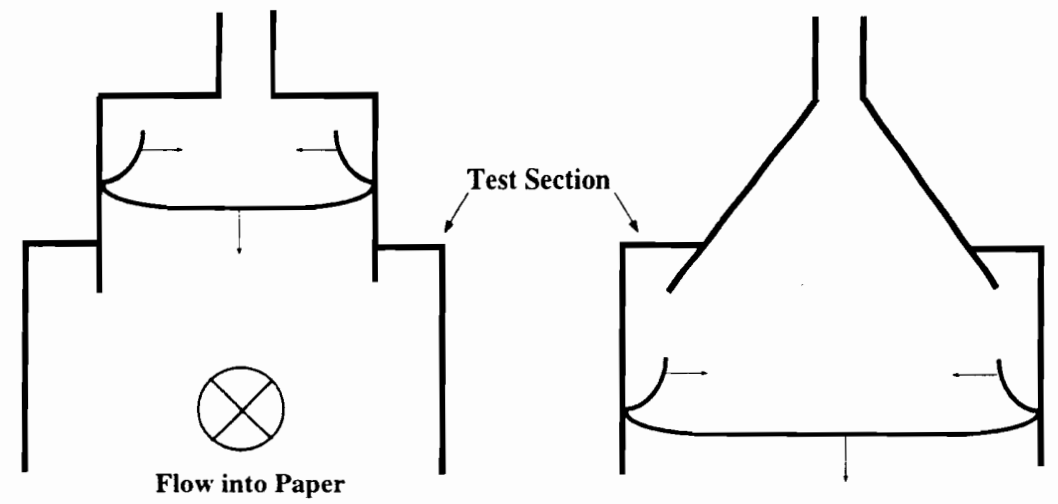

Figure 2.10: Shock-Shaper Construction [Doughty,1994]

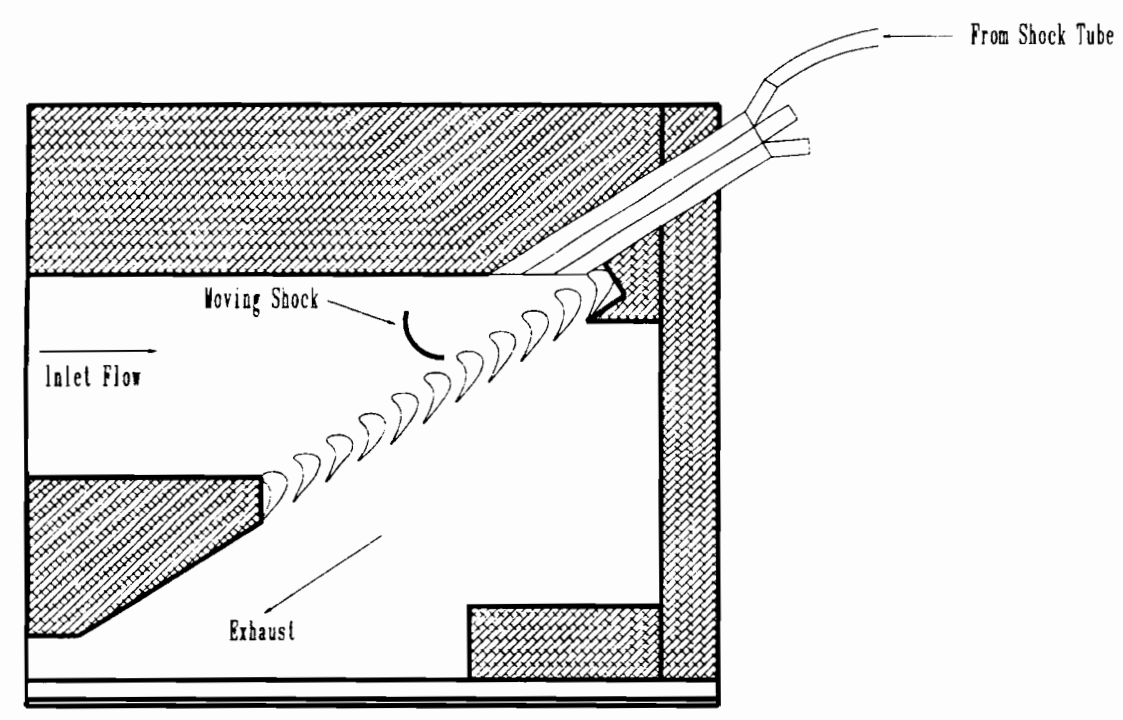

Figure 2.11: Passing Shock Orientation Within the Test Section 
opened. This isolates the flow within the heating loop. A $36 \mathrm{~kW}$ electric heater is turned on while a Dayton three-phase axial flow fan circulates air through the heating loop at approximately $2 \frac{\mathrm{m}}{\mathrm{s}}$. As the heated air passes over the two bundles of copper tubes, the flow's thermal energy is stored within the pipes. Each of the 136 $\mathrm{kg}$ bundles consists of $350,1.59 \mathrm{~cm}$ diameter and $1 \mathrm{~m}$ long copper tubes, secured by stainless steel screens downstream of each bundle, creating an effective surface area of $70 \mathrm{~m}^{2}$. The temperature of the tubes is monitored by a surface-mounted type- $\mathrm{K}$ thermocouple cemented to the end of a copper tube in the second bundle $44 \mathrm{~cm}$ from the downstream flange. The hot gas temperature is also monitored with a type- $\mathrm{K}$ thermocouple probe. This thermocouple is mounted directly in the center of the passage $0.3 \mathrm{~m}$ downstream of the second tube bundle. A third type- $\mathrm{K}$ thermocouple probe is installed on the electric heater element to monitor the heater temperature.

It takes approximately 30 minutes to initially heat the heat exchanger tubes to a temperature of $110^{\circ} \mathrm{C}\left(230^{\circ} \mathrm{F}\right)$. Subsequent heating between runs requires only 10 to 15 minutes. However, in order to match the blade temperature between subsequent runs, a Vortex tube is used to pass compressed air through the test section for 20 to 25 minutes cooling the blades to the required initial temperature. After both the tubes and the blades reach the desired temperature, the heater is turned off and the fan is allowed to run for an additional 5 minutes to equalize the temperature throughout the heat exchanger. The fan and the Vortex tube are then turned off, the two valves are opened and closed, respectively, and the test is run. Timing is very critical in this setup if repeatable experiments are to be produced. With this arrangement, flow total temperatures of $85^{\circ} \mathrm{C}$ can be achieved. 


\section{Chapter 3}

\section{Transition Study}

A necessary first step in producing any computational results for a transonic turbine cascade flow is the determination of the location of transition from laminar to turbulent flow along both the suction and pressure surfaces of the blade. Knowledge of the state of the boundary layer is also crucial for interpreting the results of experimental flowfield studies. Because of the small (though realistic) physical dimensions of the blade being studied and the low frequency vibration of the test section experienced during a tunnel run, many traditional experimental methods, such as hot film, skin friction, and high frequency surface pressure gages, etc. , for determining the transition location on the blade surface were impractical. Therefore, the use of an optical method for determining the transition location became very attractive. A fairly simple way to locate transition for cascade flow is the direct-shadow flow visualization method. In this chapter, a boundary layer transition study using the focused directshadow method is presented. In addition to the experimental shadowgraph study, the prediction of the cascade flow boundary layer transition was investigated with the application of hydrodynamic stability theory.

\subsection{Shadowgraph Study}

Optical methods are advantageous for use within a transonic cascade flow, since they enable a large field to be surveyed rapidly without introducing instrumentation that 
may induce disturbances into the flowfield. The density changes which occur in the motion of a compressible fluid past a body produce changes in the refractive index which may be observed by simple optical techniques. The refractive index, $\mathrm{n}$, of air is related to its density, $\rho$, by the Gladstone-Dole equation

$$
n-1=K \frac{\rho}{\rho_{0}}
$$

where $\rho_{0}$ is the density at normal temperature and pressure. The factor $\mathrm{K}$ is dimensionless and, for air, varies between 0.000290 and 0.000298 for the visible light spectrum. There are three basic types of optical methods which take advantage of the above fact. These three methods are interferometry, the Töpler-schlieren, and the direct-shadow methods. While they all depend on the density, they each rely on different derivatives of the density. In interferometry, the refractive index, and thus the density, in the working section is obtained by measuring the change in the velocity of the light (related to the refractive index). The schlieren method measures the first derivative of the density. Should the refractive index gradient normal to the light rays vary, the deflections of adjacent rays will diverge, giving increased illumination on the screen. The system which takes advantage of this fact is a simplified version of the schlieren system called the direct-shadow or shadowgraph method. This system, with simplifying assumptions, creates an image that is a function of the second derivative of the density [24].

\subsubsection{Apparatus}

The small boundary layer thickness produced by the high flow acceleration along the suction surface and relatively low, though realistic, Reynolds number of the blade presented a large problem in the production of usable boundary layer shadowgraph images. It was determined that in order to properly discern the state of the boundary layer, a magnified image would be required. Thus, the usual shadowgraph technique, the direct-shadow method, could not be applied, since this method is not capable of producing magnified images. However, a shadowgraph technique, termed the focuseddirect-shadow method can be used to produce magnified shadowgraph images. 
The layout of a focused-direct-shadow system is very similar to a conventional schlieren system, except for the absence of a knife edge in the focal plane of the focusing mirror. Also, the object plane is moved from the center of the test section to a position slightly outside the test section, opposite the collimating mirror, as in a conventional direct-shadow system. Due to the finite depth of focus, the illumination of the final image is the integrated result of the intensity distributions in all the planes perpendicular to the parallel light beam, just as in the direct-shadow method.

The shadowgraph pictures were taken on the fast, type 57 Polaroid film. This film was used because of its high 3000 ISO rating and high spatial resolution provided by the grainless emulsion. A Model 437B Nanopulser from Xenon was used as the light source. A Nanopulser is a type of spark light source. To try and freeze the eddies within any turbulent boundary layer region, an exposure time less than that obtainable with a mechanical shutter is required. These types of exposure times are easily obtained by discharging a capacitor across a spark gap. In the case of the Nanopulser, two electrodes, separated by an air gap are connected across a capacitor which is charged from a source of high voltage. When the voltage reaches a high enough value, the spark gap breaks down, releasing an arc discharge. The exposure time of the Model 437B is approximately $20 \mathrm{~ns}$.

A $2.034 \mathrm{~m}$ (80 in) focal length parabolic mirror was used to collimate the light before passing through the test section, and a $1.14 \mathrm{~m}$ (45 in) focal length parabolic mirror was used to focus the light onto the photographic plate. Because of the low light intensity provided by the $20 \mathrm{~ns}$ pulses from the Nanopulser, a pinhole could not be used. Therefore, great care was taken to stabilize the position of the spark source, including using a new horizontal electrode in the Nanopulser head. Also, the Nanopulser was turned so that the cross section of the light source appeared as small as possible perpendicular to a majority of the boundary layer density gradients. To obtain a sharp image, the dimensions of the light source need to be as small as possible.

A series of shadowgraphs were taken along both the suction and pressure surfaces of the blade at a magnification factor of approximately 2.5 and a nominal exit isentropic Mach number of 1.23. 


\subsubsection{Interpretation of Shadowgraphs}

The direct-shadow method has frequently been used to determine the state of boundary layers and pinpoint the position where transition from laminar to turbulent flow takes place. Direct-shadow images are characterized by an abrupt change in the thickness of the boundary layer image at the point of transition. This characteristic can be seen in the following figure, Fig. 3.4, showing typical density profiles and illumination patterns for both laminar and turbulent boundary layers. Also, as full turbulence is reached in the boundary layer, eddies can be observed within the boundary layer in the shadowgraph images. By carefully studying the series of shadowgraph images, it was determined that there was no transition along the entire pressure surface of the blade.

Also, it was apparent that the images show no transition to turbulence along the suction surface of the blade until the shock impingement. The shock impingement, as expected, then trips the laminar flow abruptly to a turbulent state. It can also be seen, from the magnified image of the trailing edge, that the flow does not separate from the blade surface until well around the slightly blunted trailing edge. Also, the pressure surface separation on the trailing edge is laminar.

Since laminar flow along the entire suction surface of a turbine blade is fairly unusual (the only example know to the author being blades studied at Oxford in [12]), a stability analysis of the suction surface of the blades used here was undertaken to support the shadowgraph results. It was also undertaken to explore the applicability of hydrodynamic stability theory to transonic turbine cascade flows in both low and high free stream turbulence levels.

\subsection{Boundary Layer Analysis}

In order to perform the hydrodynamic stability analysis, boundary layer velocity and temperature profiles, along with various wall and freestream parameters were required. These profiles needed to be smooth, since any irregularities in the profiles could cause the stability code to prematurely predict transition. The code VGBLP 


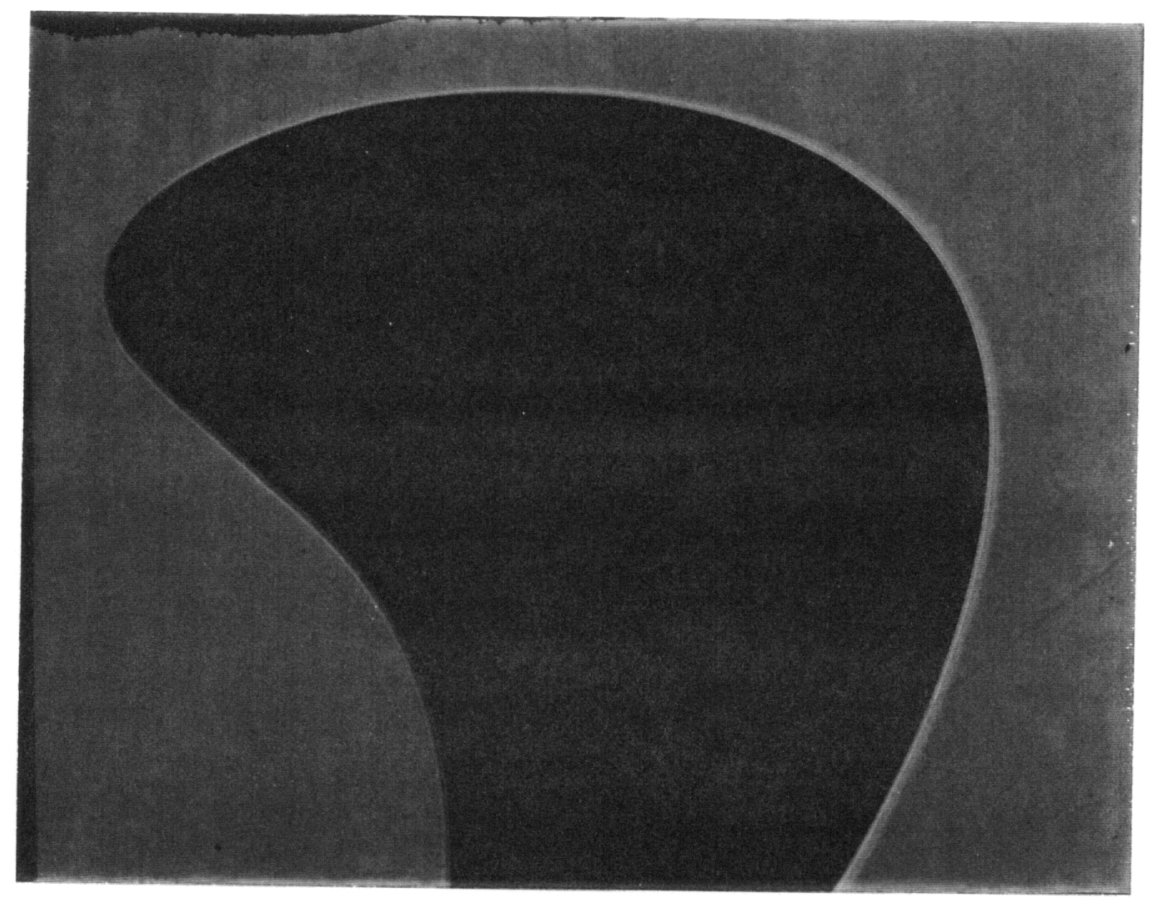

Figure 3.1: Magnified Spark-Shadowgraph Images of Boundary Layer, Low FST (A) 


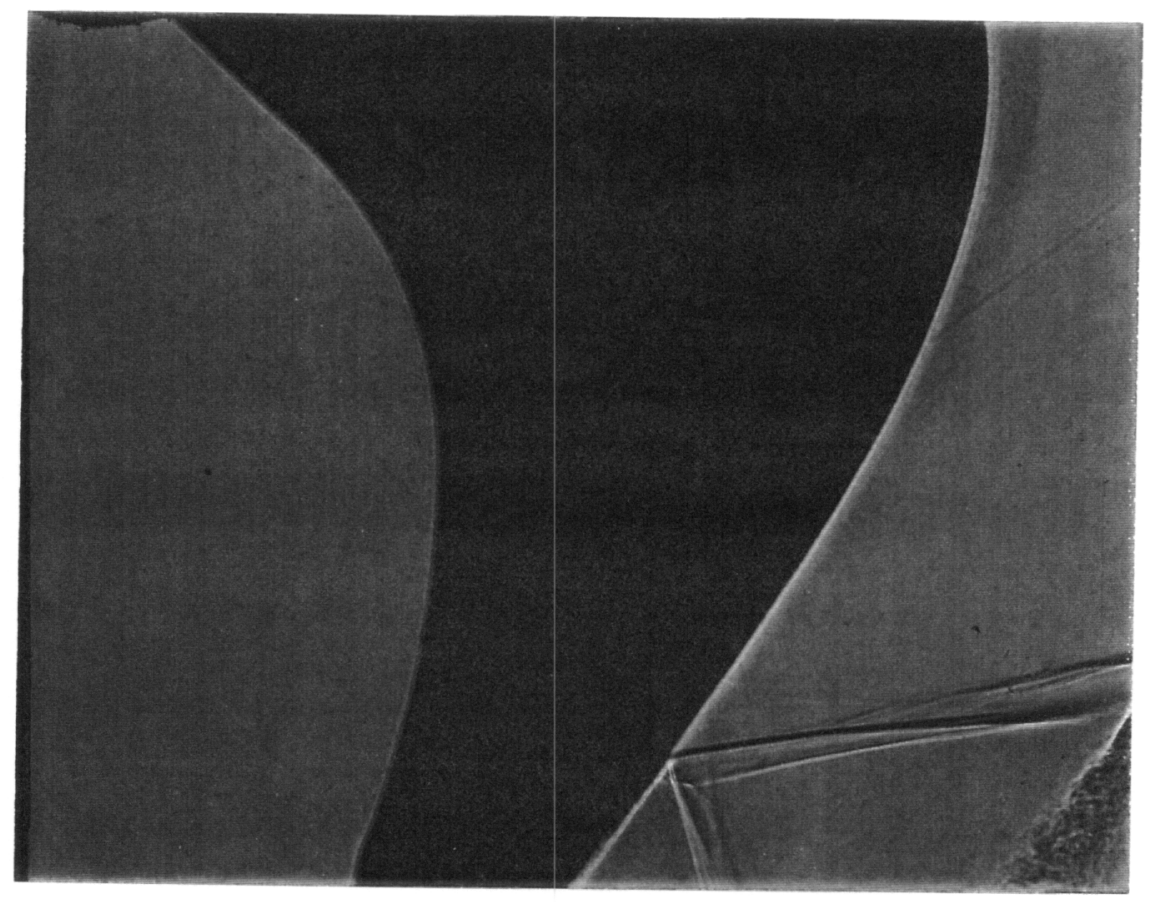

Figure 3.2: Magnified Spark-Shadowgraph Images of Boundary Layer, Low FST (B) 


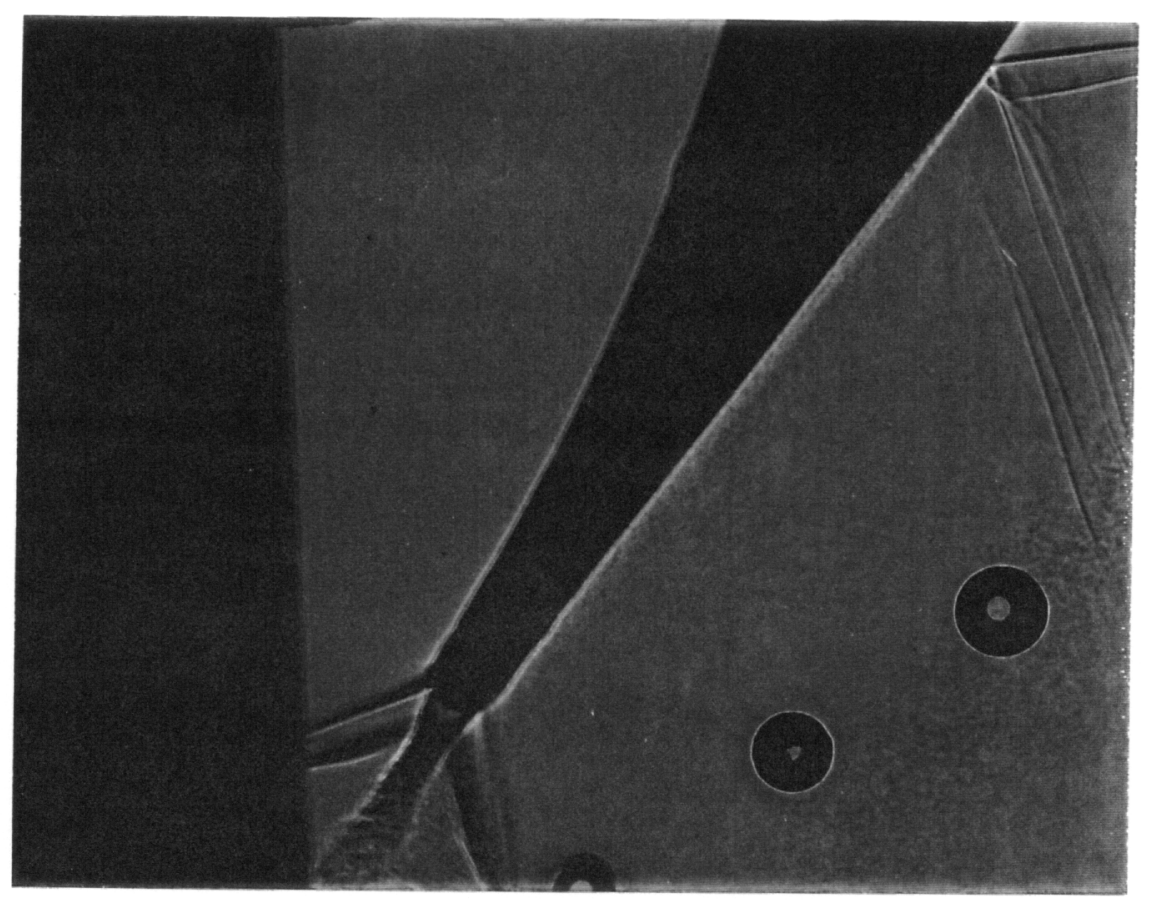

Figure 3.3: Magnified Spark-Shadowgraph Images of Boundary Layer, Low FST (C) 


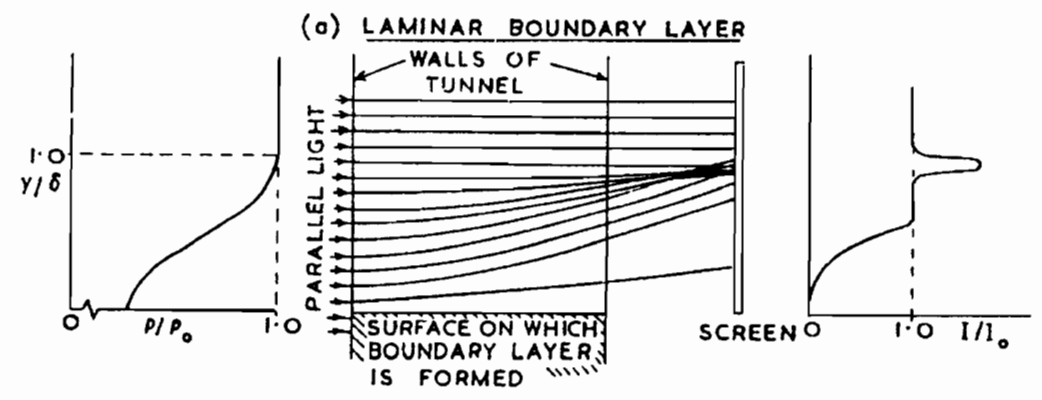

(b) TURBULENT BOUNOARY LAYER

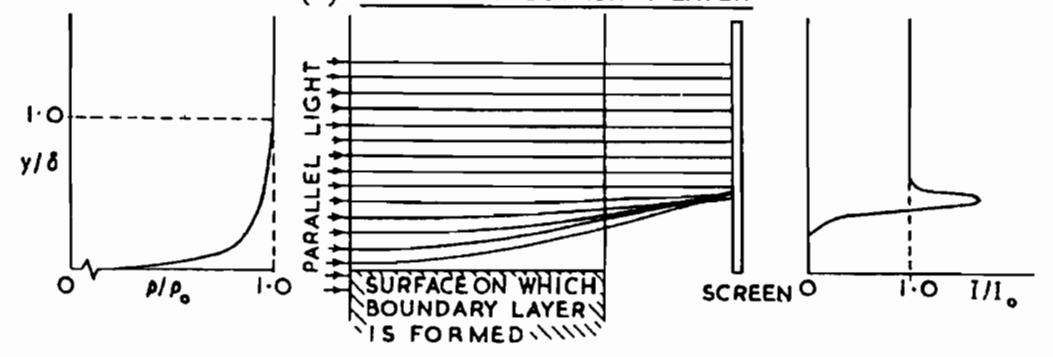

Figure 3.4: Typical Laminar and Turbulent Boundary-Layer Profiles [Holder, 1956] 
[25] was recommended because its fourth-order accuracy would produce the required smoothness in the profile curves. VGBLP initially proved difficult to work with due to machine incompatibilities. At that time, however, an improved VGBLP code, named BL2D was just being completed [26]. BL2D was written in standard For$\operatorname{tran} 77$, and it is, therefore, portable to any machine. BL2D was used to generate the required boundary layer profiles and wall parameters. The basic scheme and governing equations for the BL2D program are described below.

\subsubsection{Boundary Layer Code}

BL2D, developed at NASA Langley [26], is a fourth-order accurate Pade solution scheme for two-dimensional or axisymmetric boundary layers in speed regimes that range from low subsonic to hypersonic. Implementation of the fourth-order accurate Pade differencing scheme is similar to the procedure outlined in NASA CR-4531.

The set of equations that govern the boundary layer flow in this code can be written as:

$$
\begin{aligned}
\omega^{\prime} & =-F-2 \xi F_{\xi} \\
\omega F^{\prime}-\left(l_{1} F^{\prime}\right)^{\prime} & =-2 \xi F F_{\xi}-\beta\left(F^{2}-H\right) \\
\omega H^{\prime}-\left(l_{2} H^{\prime}\right)^{\prime} & =-2 \xi F H_{\xi}+\alpha l_{1}\left(F^{\prime}\right)^{2}
\end{aligned}
$$

where $\omega$ is being substituted for $U, \zeta$ is the transformed normal coordinate, the prime symbol ' is for the normal derivative $\left(\frac{\partial}{\partial \zeta}\right)$ and the subscript $\xi$ indicates a partial derivative in the $\xi$ direction.

Also the following notation is used,

$$
\begin{gathered}
l_{1}=t^{2 j} l \bar{\epsilon} \\
l_{2}=t^{2 j} l \tilde{\epsilon} \\
L=F^{\prime}
\end{gathered}
$$




$$
I=H^{\prime}
$$

Note that in the above equations $\mathrm{j}=0$ or 1 for two-dimensional planar or axisymmetric flows, respectively; $l=\frac{\rho \mu}{\rho_{e} \mu_{e}} ; \Gamma$ is the streamwise intermittency distribution; $N_{P r}$ is the laminar value of the Prandtl number; $\bar{\epsilon}$ is the eddy viscosity function $\left(1+\frac{\epsilon}{\mu} \Gamma\right)$; $\tilde{\epsilon}$ is the eddy viscosity function $\frac{1}{N_{P r}}\left(1+\frac{\epsilon}{\mu} \frac{N_{P r}}{N_{P r, t}} \Gamma\right) ; \mathrm{t}$ is the transverse curvature term $\frac{r}{r_{\omega}} ; \beta=\frac{2 \xi}{u_{e}}\left(u_{e}\right)_{\xi} ;$ and $\alpha=(\gamma-1) M_{e}^{2}$.

In vector form, denoting

$$
Q=\left(\begin{array}{c}
F \\
F \omega-l_{1} L \\
H \\
H \omega-l_{2} I
\end{array}\right)
$$

the governing equations can be written in the following form.

$$
Q^{\prime}=\left(\begin{array}{c}
L \\
-F^{2}(1+\beta)-4 \xi F F_{\xi}+\beta H \\
I \\
-2 \xi(F H)_{\xi}-F H+\alpha l_{1} L^{2}
\end{array}\right)
$$

The second derivative, $\mathrm{Q}^{\prime \prime}$, required in the differencing scheme is

$$
Q^{\prime \prime}=\left(\begin{array}{c}
\frac{1}{l_{1}}\left[F\left(2 \xi F_{\xi}+\beta F\right)+L\left(\omega-l_{1}^{\prime}\right)-\beta H\right] \\
-2 F L(1+\beta)-4 \xi\left(F L_{\xi}+L F_{\xi}\right)+\beta I \\
\frac{1}{l_{2}}\left[2 \xi F H_{\xi}-\alpha l_{1} L^{2}+I\left(\omega-l_{2}^{\prime}\right)\right] \\
-2 \xi(F I+H L)_{\xi}-(F I+H L)+2 \alpha L\left[F\left(2 \xi F_{\xi}+\beta F\right)+L \omega-\beta H\right]
\end{array}\right)
$$

The discretization of the vector $\mathrm{Q}$ is now described. Again following the procedure given in NASA CR-4531, the fourth-order Pade differencing formula (applied in the normal direction) is a two-point compact scheme defined in terms of the variable $Q$ and its two higher derivatives. Assuming that $i$ remains constant and that $k$ is the normal direction index, then the discretization at the midpoint of $k$ and $(k-1)$ is written as 


$$
\begin{gathered}
Q_{k}-Q_{k-1}-\frac{\triangle \zeta}{2}\left(Q_{k}^{\prime}+Q_{k-1}^{\prime \prime}\right)+\frac{\triangle \zeta^{2}}{12}\left(Q_{k}^{\prime \prime}-Q_{k-1}^{\prime \prime}\right)+O\left(\triangle \zeta^{5}\right)=0 \\
\triangle \zeta=\zeta_{k}-\zeta_{k-1}
\end{gathered}
$$

The $\xi$ differencing, in keeping with the parabolic nature of the equations is usually obtained using a second-order accurate three-point upwind-differenced formula.

$$
\begin{gathered}
\left(f_{\xi}\right)_{i}=a_{1} f_{i}+a_{2} f_{i-1}+a_{3} f_{i-2} \\
a_{1}=\frac{\left(\triangle \xi_{i}^{2}-\triangle \xi_{i-1}^{2}\right)}{\triangle} ; a_{2}=\frac{-\triangle \xi_{i}^{2}}{\triangle} ; a_{3}=\frac{\triangle \xi_{i-1}^{2}}{\triangle} \\
\triangle=\triangle \xi_{i} \triangle \xi_{i-1}\left(\triangle \xi_{i}+\triangle \xi_{i-1}\right) ; \triangle \xi_{i}=\xi_{i}-\xi_{i-1} ; \triangle \xi_{i-1}=\xi_{i-1}-\xi_{i-2}
\end{gathered}
$$

The initial (starting) velocity and temperature profiles required for the parabolic marching solution are produced either from similarity assumptions or two-dimensional stagnation point assumptions (depending on tip conditions) as is traditionally done in boundary layer flows. Using the equations derived by Falkner and Skan (1930) for producing similar boundary layer solutions in inviscid flow over a wedge with an opening angle of $\beta \pi=\frac{2 m \pi}{m+1}$ where $U_{e}(x)=U_{1} x^{m}$, the planar stagnation point solution $(\beta=m=1)$ provides the initial conditions for nonsimilar boundary layer calculations over blunt-nosed bodies of general shapes [27]. Also, since the three-point upwind differencing scheme would require two points upstream of the initial profile, a first order formula is used over the first few marching stations.

The solution vector is given by

$$
S=\left(\begin{array}{c}
F \\
L \\
H \\
I
\end{array}\right)
$$


Since the equations are nonlinear in S, Newton linearization is used to create a simple linear matrix inversion problem. If the superscript $n$ is used to denote the current iteration stage, then,

$$
\delta S=S^{n}-S^{n-1}
$$

The inversion of the resulting block-tridiagonal system by standard routines produces the linearized solution for $\delta S$. The previous solution is updated and the process is repeated until convergence is obtained. The BL2D code was used to produce all laminar boundary layer profiles for the hydrodynamic stability calculations.

\subsubsection{Boundary Layer Code Input}

The boundary layer analysis was performed for three separate cases using the General Electric supplied blade profile; 1) a wall heated case, 2) an adiabatic wall case and 3) a wall-cooled case. The adiabatic wall case approximates the baseline case for all of our experimental work. Also, because of the new tunnel flow heating capabilities described in the Introduction, we can run a wall-cooled experiment to more closely match typical engine conditions. The cooled wall calculation was run to match typical conditions during a "heated flow" experiment.

The wall-heated case was run as a check to the stability code. Where a wall-cooled case would tend to have a stabilizing effect on the boundary layer, the wall-heated case (which we do not run in the tunnel) would tend to have a destabilizing effect on the boundary layer. Therefore, by numerically simulating this condition, we can check to see if this destabilizing effect is realized in the stability calculations. How the run conditions for varied for each case are shown in Table 3.1. Run conditions consistent to all three cases are shown in Table 3.2.

The static pressure profile for the studied blade was provided by General Electric in terms of isentropic Mach numbers. It should be noted that, in order to provide the correct input for the stability calculations, all three cases were run at fully laminar conditions. Also, since BL2D cannot calculate through a shock impingement, the supplied pressure profile is cut off before the sharp pressure rise following the shock 
impingement from the neighboring upstream blade.

\subsubsection{Boundary Layer Results}

Figure 3.5 shows the surface isentropic Mach number distribution for all the computed cases superimposed on the General Electric supplied profile. This is shown primarily to ensure that the blade static pressure profile was properly supplied to the boundary layer code. One important observation to make is that the blade profile always supplies either a favorable or a zero pressure gradient to the boundary layer flow. This is an important factor in the stability of a boundary layer flow, as will be discussed at length later.

Figure 3.6 shows the calculated boundary layer thickness profile in $\mathrm{mm}$ versus surface distance in $\mathrm{m}$. As was noted from the spark shadowgraphs, the boundary layer is extremely thin. In all three cases, the boundary layer never reaches a thickness of $0.25 \mathrm{~mm}$. This small boundary layer thickness can most likely be attributed to the large flow acceleration provided by the blade suction surface.

Figure 3.7 provides the pressure gradient parameter distribution versus the distance along the blade's suction surface. The pressure gradient parameter is a measure of the flow acceleration along the blade surface and is given by the equation

$$
\beta=2 \xi \frac{\partial u_{e}}{\partial \xi}
$$

where $\beta$ is the pressure gradient parameter and $\xi$ is the local coordinate along the blade surface. If $\beta>0$ the pressure gradient is favorable, where as, if $\beta<0$ the pressure gradient is adverse.

Another feature of note in Fig. 3.6 is the fact that while the adiabatic wall and heated wall boundary layer thickness are similar, the cooled wall case has a significantly thicker boundary layer. This is somewhat counter-intuitive since cooled wall boundary layers are typically thinner than heated wall boundary layers for the same free stream conditions. However, since we vary the conditions in the tunnel by altering the free stream temperature and holding the wall conditions constant, the fact that the cooled wall case has a thicker boundary layer is consistent with the 
Table 3.1: Case Specific BL2D Input Flow Conditions

\begin{tabular}{|c|c||c|c|c|}
\hline BL2D INPUT & DESCRIPTION & ADIABATIC & COOLED & HEATED \\
\hline \hline AMACH & Inlet Mach \# & 0.36 & 0.36 & 0.36 \\
\hline PTS & Inlet Total Pres. (psf) & 4666.15 & 4666.15 & 4666.15 \\
\hline TTS & Inlet Total Temp. (R) & 504.0 & 815.4 & 504.0 \\
\hline TWSE & Wall Temp. (R) & adiabatic & 671.4 & 675.0 \\
\hline
\end{tabular}

Table 3.2: General BL2D Input Conditions

\begin{tabular}{|c|c||c|}
\hline BL2D INPUT & DESCRIPTION & VALUE \\
\hline \hline NZI & Number of Normal Grid Points & 181 \\
\hline AK & Grid Stretching Parameter & 1.05 \\
\hline IACC & Order of Accuracy & 4 \\
\hline DFPTOL & Convergence criterion for $\triangle f^{\prime}$ & $0.7 \times 10^{-8}$ \\
\hline DHPTOL & Convergence criterion for $\triangle h^{\prime}$ & $0.7 \times 10^{-8}$ \\
\hline RSTAR & Gas Constant & $286.96 \frac{\mathrm{m}^{2}}{\sec ^{2} K}$ \\
\hline
\end{tabular}

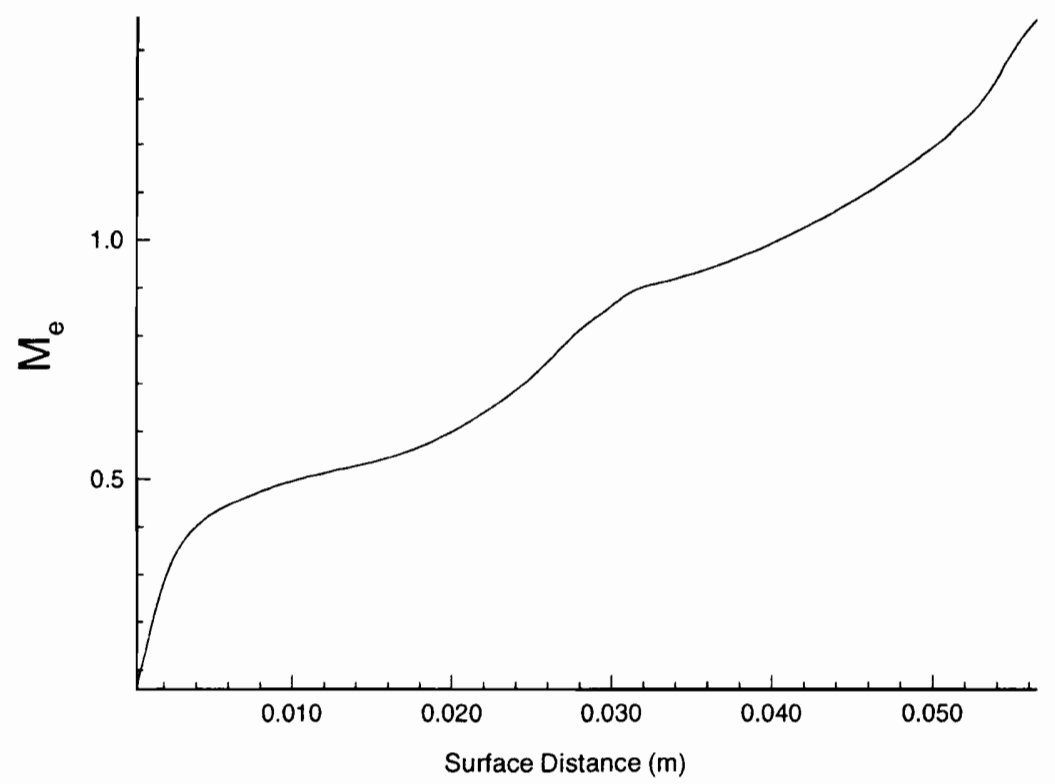

Figure 3.5: Steady Surface Isentropic Mach Number Distribution - All Cases 


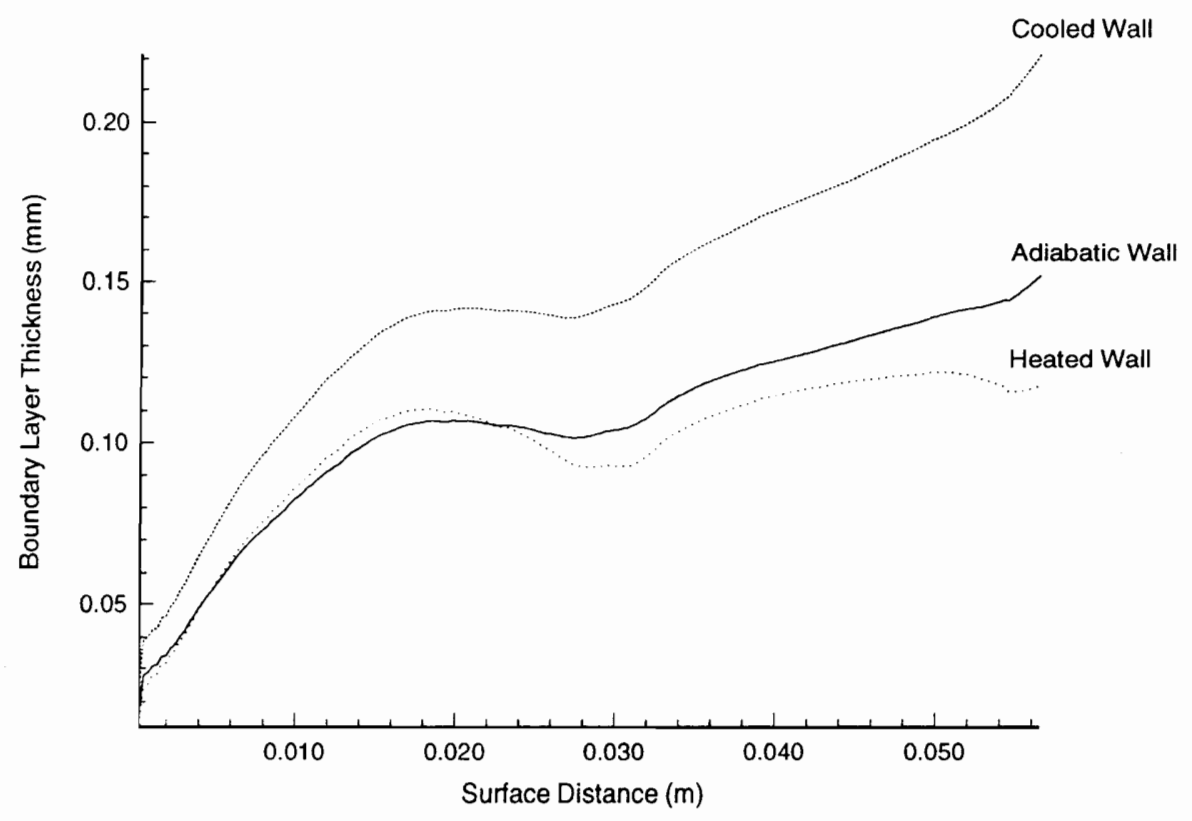

Figure 3.6: Steady Boundary Layer Thickness - All Cases

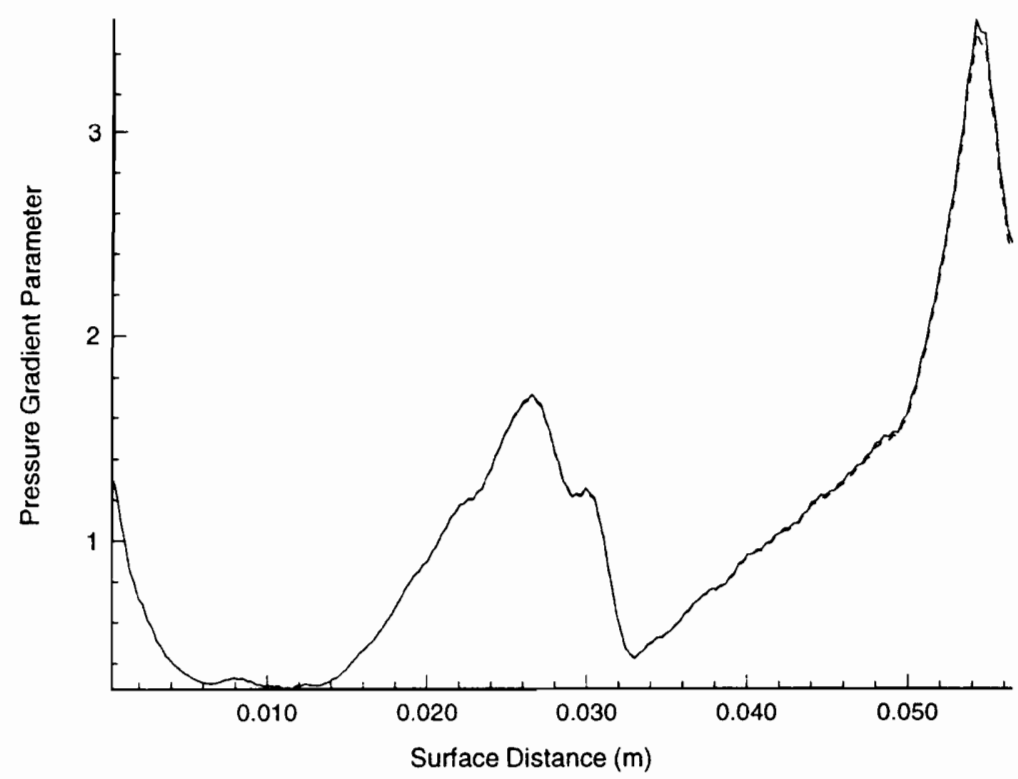

Figure 3.7: Steady Pressure Gradient Parameter Distribution - All Cases 
density variation produced by altering the upstream temperature.

Figure 3.8 depicts a very crude but simple method for predicting the location of transition developed by R. Michel in 1952 [27]. By correlating the observed transition location for low-speed flows with their momentum thickness Reynolds number, he developed the following equation for locating transition.

$$
\operatorname{Re}_{\theta_{\text {trans }}} \approx 2.9 R e_{x_{\text {trans }}^{0.4}}^{0 .}
$$

here, taking $\mathrm{s}$ for $\mathrm{x}$, it is easily seen that according to this method no transition is predicted for the adiabatic wall case on the suction surface of this blade profile.

Plots for the momentum thickness Reynolds number (for all three cases), Fig. 3.9, and the wall friction coefficient, Fig. 3.10, are also presented.

\subsection{Stability Analysis}

The mean flow profiles provided by the BL2D code were analyzed using quasi-parallel, compressible linear stability theory. At the low Mach numbers encountered here, no supersonic region exists near the wall relative to the phase velocity, $\mathrm{C}_{s}$, hence, no second-mode instability exists. The lowest Mach number at which the unstable second-mode region has been located at finite Reynolds numbers is $M=3$. Therefore, only disturbance frequencies which tend to produce first-mode instabilities were tested. Frequencies were tested from 10 to $100 \mathrm{kHz}$ in increments of $10 \mathrm{kHz}$, with the increments being reduced to $5 \mathrm{kHz}$ if the global criterion for instability was met.

\subsubsection{Hydrodynamic Stability Theory Background}

Mechanisms for laminar to turbulent transition include linear instability, bypass transition, Görtler instability and cross-flow instability. Since small disturbances are always present in the background of any flow, the main question in determining the stability of a flow is, "Do these disturbances grow or are they damped out?" Since the analysis of the full, unsteady, three-dimensional case is difficult, some simplifying 


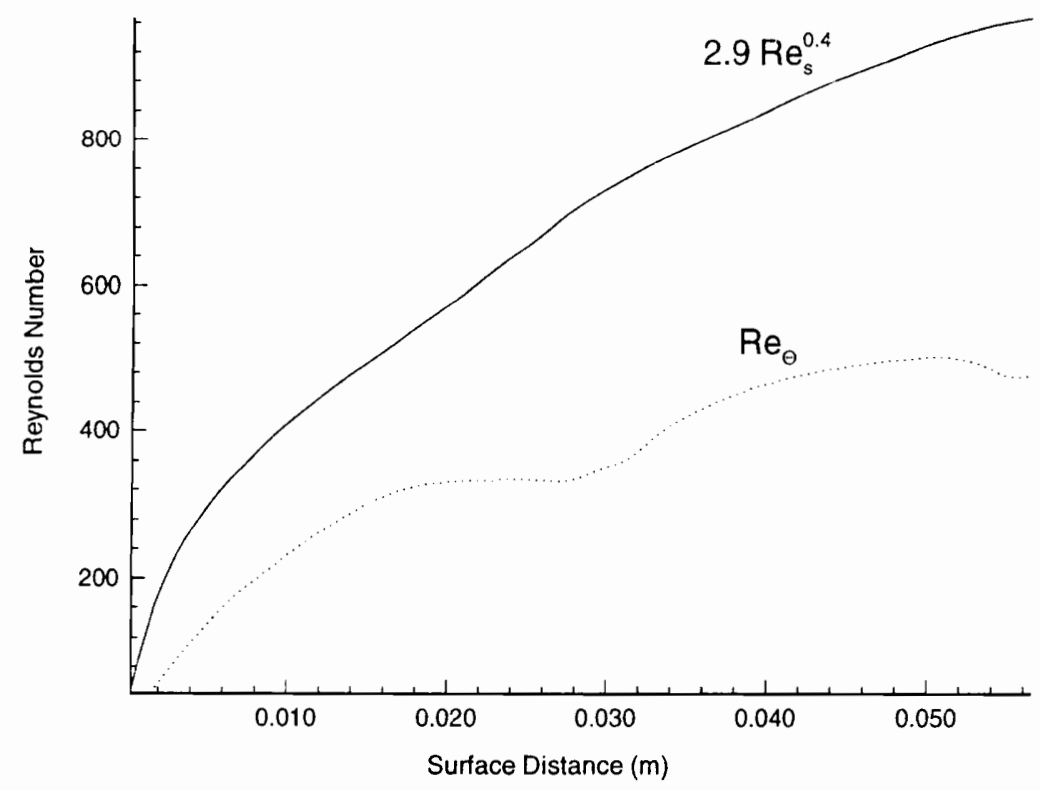

Figure 3.8: Steady, Adiabatic Wall Boundary Layer Michel Plot

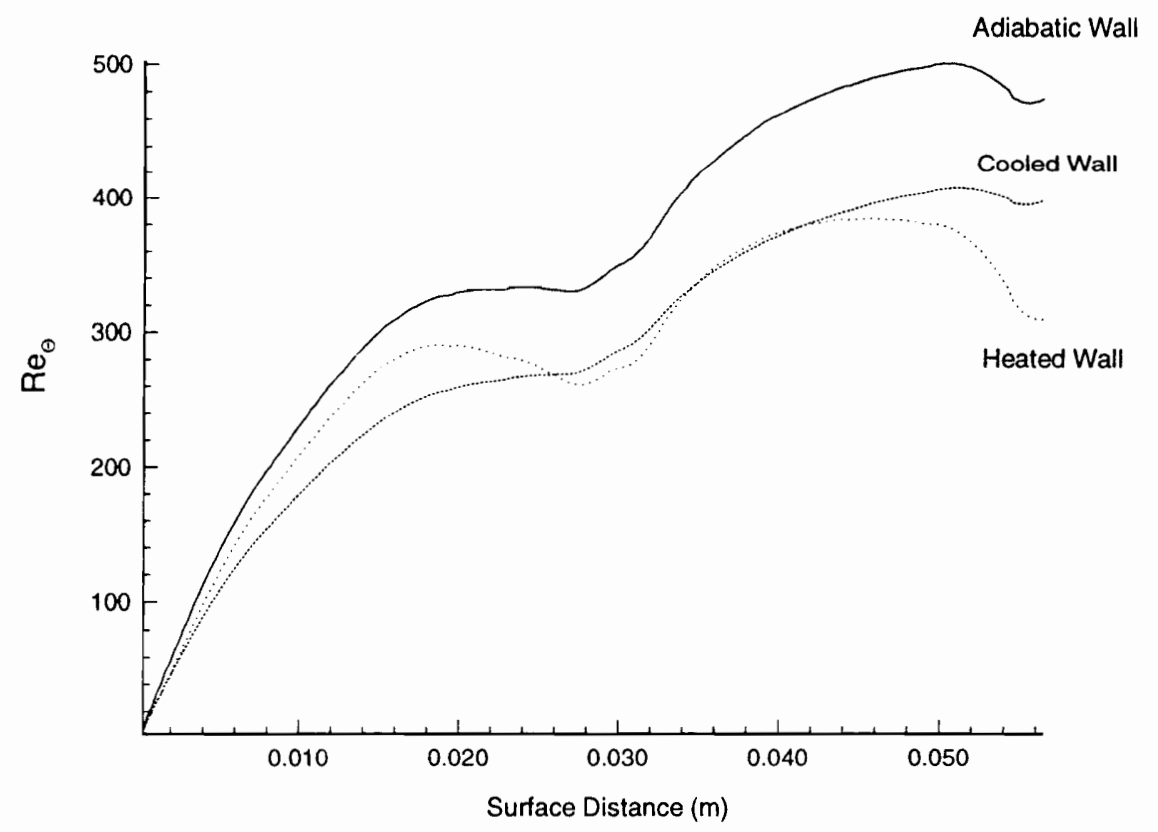

Figure 3.9: Steady Momentum Thickness Reynolds Number - All Cases 
assumptions are generally made in this theory. First, since two-dimensional disturbances are always less stable than three-dimensional disturbances in subsonic flow, only the 2-D case is examined. The other main assumptions in this theory are that the disturbances are small, the mean flow is only a function of the transverse coordinate and the disturbances can be described by the stream function in the form

$$
\Psi(y)=\phi(y) e^{i(\alpha x-\omega t)}
$$

where $\phi$ is the complex amplitude function of the disturbance flow variable $\tilde{v}, \alpha$ is the complex wave number of the disturbance and $\omega$ is the complex phase velocity. With Eq. 3.18 and the additional assumptions (made here for the moment for the purposes of clarity of presentation) of laminar, incompressible and constant property flow, the famous Orr-Sommerfeld equation can be produced from the Navier-Stokes equations.

$$
\left[(\alpha U-\omega)\left(\phi^{\prime \prime}-\alpha^{2} \phi\right)-\alpha U^{\prime \prime} \phi\right]=-\frac{i}{R}\left(\phi^{(i v)}-2 \alpha^{2} \phi^{\prime \prime}+\alpha^{4} \phi\right)
$$

It is possible to simplify Eq. 3.19 by neglecting the viscous terms, which are preceded by the exponentially small term of $\frac{1}{R}$. The resulting equation is know as Rayleigh's equation.

$$
(U-c)\left(\phi^{\prime \prime}-\alpha^{2} \phi\right)-U^{\prime \prime} \phi=0
$$

Note that the stability equation has been reduced from fourth to second order. Using this equation, Lord Rayleigh derived two basic theorems dealing with the stability of laminar velocity profiles. The first theorem dealt with velocity inflection points. It states:

THEOREM 1: The point-of-inflection criterion asserts that velocity profiles which possess a point of inflection are unstable.

This theorem is very fundamental to boundary layer theory, in general, and hydrodynamic stability theory, in particular. In boundary layer theory, it provides a rough classification for all laminar flow profiles. Secondly, it provides an explanation for the destabilizing effect of adverse pressure gradients of boundary layer flows. According to boundary layer theory, velocity profiles are free from points of inflection when the 
pressure decreases in the streamwise direction, whereas velocity profiles in the presence of an adverse pressure gradient always possess them. This fact is very important in the case of the turbine blade being studied, because the blade has a strong negative pressure gradient along the entire suction surface (up until the trailing edge shock inpengment). This would suggest that the boundary layer would have a tendency to remain laminar along the entire suction surface (again up until the trailing edge shock impingement.)

The second theory developed by Rayleigh states:

THEOREM 2: The velocity of propagation of neutral disturbances $\left(c_{i}=0\right)$ in a boundary layer is smaller than the maximum velocity of the mean flow.

It can be easily shown that this creates a mathematical singularity indicating that the viscosity effect on the equations of motion must not be neglected in the neighborhood of the critical layer.

The two theorems both show the importance of knowing to sufficient accuracy, not only the mean flow, but also the second derivative of the mean flow. Thus the choice of boundary layer code to produce the mean flow profiles is critical. The BL2D code provides fourth order accuracy for the mean profiles.

There are two distinct approaches to linear stability theory, temporal and spatial. In steady mean flow, the amplitude of a disturbance at a fixed point in the flow is independent of time and changes only with transverse distance. In this case, spatial theory gives this amplitude change in a more direct manner than temporal theory.

The compressible analog of the Orr-Sommerfeld equation is a coupled set of five ordinary differential equations. These include three second-order momentum equations, one second-order energy equation and one first-order continuity equation. These may be further reduced to a set of eight first-order ordinary differential equations following the approach first used by Lees and Lin [28].

Below, the approach given by Malik for the development of the compressible linear stability equations is outlined. 


\subsubsection{Stability Code}

The stability analysis was performed with the $\mathrm{e}^{\text {Malik }}$ code. This is a transitionprediction code for 2-D and axisymmetric flows developed in 1992 by Dr. Malik of NASA Langley Research Center [29]. The code uses quasi-parallel spatial stability theory and can treat subsonic to hypersonic flows including equilibrium real gas effects. First, second, third, etc..., mode disturbances as well as Görtler vortex instabilities can be investigated.

Starting from the Navier-Stokes equations governing the flow of a viscous compressible ideal gas, the governing equations for the steady mean flow may be derived by invoking the well known boundary layer assumptions of Prandtl. The stability equations are then derived by assuming small disturbances superimposed on the mean flow and substituting in the Navier-Stokes equations.

The coordinate system used by Malik in the derivation of the compressible linear stability equations code is a simple Cartesian coordinate system where $x$ is the streamwise coordinate, $z$ is the spanwise coordinate and $y$ is the coordinate normal to the solid boundary. He assumes that all lengths are scaled by a reference length $l$, velocities by $u_{e}$, the density by $\rho_{e}$, pressure by $\rho_{e} u_{e}^{2}$, time by $\frac{l}{u_{e}}$ and all other variables by their corresponding boundary layer edge values. The instantaneous values of the velocities, $u, v, w$; pressure, $p$; temperature, $\tau$; and the density, $\rho$ may be represented by a combination of their mean and fluctuating values, i.e.,

$$
\begin{array}{llll}
u=\bar{U}+\tilde{u}, & v=\bar{V}+\tilde{v}, & w=\bar{W}+\tilde{w} \\
p=\bar{P}+\tilde{p}, & \tau=\bar{T}+\tilde{T}, & \rho=\bar{\rho}+\tilde{\rho} \\
\mu=\bar{\mu}+\tilde{\mu}, & \Lambda=\bar{\lambda}+\tilde{\lambda}, & \kappa=\bar{\kappa}+\tilde{\kappa}
\end{array}
$$

The linearized perturbation equations are produced by substituting these decomposed values into the nondimensional form of the Navier-Stokes equations governing the flow of a viscous compressible ideal gas. Now, by invoking the quasi-parallel flow assumption (dropping the bars),

$$
U=U(y), \quad V=0, \quad W=W(y), \quad T=T(y), \quad \rho=\rho(y)
$$


using the equation of state $\tilde{\rho}=\gamma M^{2} \frac{\tilde{p}}{T}-\frac{\tilde{T}}{T^{2}}$ to eliminate the density $\tilde{\rho}$ and writing

$$
\tilde{\mu}=\frac{d \mu}{d T} \tilde{T}, \quad \tilde{\lambda}=\frac{d \lambda}{d T} \tilde{T}, \quad \tilde{k}=\frac{d k}{d T} \tilde{T}
$$

the following five stability equations can be derived.

$$
\begin{aligned}
& \frac{\frac{\partial \tilde{u}}{\partial t}+U \frac{\partial \tilde{u}}{\partial x}+\tilde{v} \frac{d U}{d y}+W \frac{\partial \tilde{u}}{\partial z}}{T}=-\frac{\partial \tilde{p}}{\partial x}+\frac{\mu}{R}\left[l_{2} \frac{\partial^{2} \tilde{u}}{\partial x^{2}}+l_{1}\left(\frac{\partial^{2} \tilde{v}}{\partial x \partial y}+\frac{\partial^{2} \tilde{w}}{\partial x \partial z}\right)\right. \\
& +\frac{\partial^{2} \tilde{u}}{\partial y^{2}}+\frac{\partial^{2} \tilde{u}}{\partial z^{2}}+\frac{1}{\mu} \frac{d \mu}{d T} \frac{d T}{d y}\left(\frac{\partial \tilde{u}}{\partial y}+\frac{\partial \tilde{v}}{\partial x}\right) \\
& +\frac{1}{\mu} \frac{d \mu}{d T}\left(\frac{d^{2} U}{d y^{2}} \tilde{T}+\frac{d U}{d y} \frac{\partial \tilde{T}}{\partial y}\right) \\
& \left.+\frac{1}{\mu} \frac{d^{2} \mu}{d T^{2}} \frac{d T}{d y} \frac{d U}{d y} \tilde{T}\right] \\
& \frac{\frac{\partial \tilde{v}}{\partial t}+U \frac{\partial \tilde{v}}{\partial x}+W \frac{\delta \tilde{v}}{\delta z}}{T}=-\frac{\partial \tilde{p}}{\partial y}+\frac{\mu}{R}\left[\frac{\partial^{2} \tilde{v}}{\partial x^{2}}+l_{1}\left(\frac{\partial^{2} \tilde{u}}{\partial x \partial y}+\frac{\partial^{2} \tilde{w}}{\partial y \partial z}\right)\right. \\
& +l_{2} \frac{\partial^{2} \tilde{v}}{\partial y^{2}}+\frac{\partial^{2} \tilde{v}}{\partial z^{2}}+\frac{1}{\mu} \frac{d \mu}{d T}\left(\frac{\partial \tilde{T}}{\partial x} \frac{d U}{d y}+\frac{\partial \tilde{T}}{d z} \frac{d W}{d y}\right) \\
& \left.+\frac{1}{\mu} \frac{d \mu}{d T} \frac{d T}{d y}\left\{l_{0}\left(\frac{\partial \tilde{u}}{\partial x}+\frac{\partial \tilde{w}}{\partial z}\right)+l_{2} \frac{\partial \tilde{v}}{\partial y}\right\}\right]
\end{aligned}
$$

$$
\begin{aligned}
& \frac{\partial \tilde{w}}{\partial t}+U \frac{\partial \bar{w}}{\partial x}+\tilde{v} \frac{d W}{d y}+W \frac{\partial \tilde{w}}{\partial z}=-\frac{\partial \tilde{p}}{\partial z}+\frac{\mu}{R}\left[\frac{\partial^{2} \tilde{w}}{\partial x^{2}}+l_{1}\left(\frac{\partial^{2} \tilde{u}}{\partial x \partial z}+\frac{\partial^{2} \tilde{v}}{\partial y \partial z}\right)\right. \\
&+\frac{\partial^{2} \tilde{w}}{\partial y^{2}}+l_{2} \frac{\partial^{2} \tilde{w}}{\partial z^{2}}+\frac{1}{\mu} \frac{d \mu}{d T} \frac{d T}{d y}\left(\frac{\partial \tilde{v}}{\partial z}+\frac{\partial \tilde{w}}{\partial y}\right) \\
&+\frac{1}{\mu} \frac{d \mu}{d T}\left(\frac{d^{2} W}{d y^{2}} \tilde{T}+\frac{d W}{d y} \frac{\partial \tilde{T}}{\partial y}\right) \\
&\left.+\frac{1}{\mu} \frac{d^{2} \mu}{d T^{2}} \frac{d T}{d y} \frac{d W}{d y} \tilde{T}\right] \\
& \frac{\gamma M^{2}}{T} \frac{\partial \tilde{p}}{\partial t}-\frac{1}{T^{2}} \frac{\partial \tilde{T}}{\partial t}+ \frac{1}{T} \frac{\partial \tilde{u}}{\partial x}+U\left(\frac{\gamma M^{2}}{T} \frac{\partial \tilde{p}}{\partial x}-\frac{1}{T^{2}} \frac{\partial \tilde{T}}{\partial x}\right) \\
&+\frac{1}{T} \frac{\partial \tilde{v}}{\partial y}-\frac{1}{T^{2}} \frac{d T}{d y} \tilde{v}+\frac{1}{T} \frac{\partial \tilde{w}}{\partial z}+W\left(\frac{\gamma M^{2}}{T} \frac{\partial \tilde{p}}{\partial z}-\frac{1}{T^{2}} \frac{\partial \tilde{T}}{\partial z}\right)=0
\end{aligned}
$$




$$
\begin{aligned}
\frac{\partial \tilde{T}}{\partial t}+U \frac{\partial \tilde{T}}{\partial x}+\tilde{v} \frac{d T}{d y}+W \frac{\partial \tilde{T}}{\partial z}= & (\gamma-1) M^{2}\left[\frac{\partial \tilde{p}}{\partial t}+U \frac{\partial \tilde{p}}{\partial x}+W \frac{\partial \tilde{p}}{\partial z}\right] \\
& +\frac{\mu}{R N_{P r}}\left[\frac{\partial^{2} \tilde{T}}{\partial x^{2}}+\frac{\partial^{2} \tilde{T}}{\partial y^{2}}+\frac{\partial^{2} \tilde{T}}{\partial z^{2}}\right. \\
& +\frac{2}{k} \frac{d K}{d T} \frac{d T}{d y} \frac{\partial \tilde{T}}{\partial y} \\
& \left.+\left(\frac{1}{k} \frac{d K}{d T} \frac{d^{2} T}{d y^{2}}+\frac{1}{k} \frac{d^{2} k}{d T^{2}}\left(\frac{d T}{d y}\right)^{2}\right) \tilde{T}\right] \\
& +(\gamma-1) M^{2} \frac{\mu}{R}\left[2 \frac{d U}{d y}\left(\frac{\partial \tilde{u}}{\partial y}+\frac{\partial \tilde{v}}{\partial x}\right)\right. \\
& +2 \frac{d W}{d y}\left(\frac{\partial \tilde{v}}{\partial z}+\frac{\partial \tilde{w}}{\partial y}\right) \\
& \left.+\frac{1}{\mu} \frac{d \mu}{d T}\left(\left(\frac{d U}{d y}\right)^{2}+\left(\frac{d W}{d y}\right)^{2}\right)\right]
\end{aligned}
$$

where $N_{P r}$ is the variable Prandtl number defined by $N_{P r}=\frac{\mu c_{p}}{k}$.

As in the Orr-Sommerfeld derivation, the velocity, pressure and temperature fluctuations are represented by harmonic waves, given by;

$$
\begin{aligned}
(\tilde{u}, \tilde{v}, \tilde{w}) & =[\hat{u}(y), \hat{v}(y), \hat{w}] e^{i(\alpha x+\beta z-\omega t)} \\
\tilde{p} & =\hat{p}(y) e^{i(\alpha x+\beta z-\omega t)} \\
\tilde{T} & =\hat{T}(y) e^{i(\alpha x+\beta z-\omega t)}
\end{aligned}
$$

where $\alpha, \beta$ are the wavenumbers and $\omega$ is the frequency of the free stream disturbance which, in general, are all complex.

Using these substitutions, it can be shown that the above equations governing the behavior of linear disturbances in a boundary layer flow can be reduced to a system of five ordinary differential equations of the following form:

$$
\left(A D^{2}+B D+C\right) \Phi=0
$$

where $\mathrm{D}=\frac{d}{d y}$ and where $\Phi$ is a five-element vector defined by 


$$
\{\hat{u}, \hat{v}, \hat{p}, \hat{T}, \hat{w}\}^{T}
$$

noting that for two-dimensional flow the mean transverse velocity (W) is set to zero and the order of the system can be reduced by dropping the $\hat{w}$ momentum equation.

The boundary conditions are given as

$$
\begin{gathered}
y=0 ; \quad \phi_{1}=\phi_{2}=\phi_{4}=\phi_{5}=0 \\
y \rightarrow \infty ; \quad \phi_{1}, \phi_{2}, \phi_{4}, \phi_{5} \rightarrow 0
\end{gathered}
$$

Thus, the central problem of determining the hydrodynamic stability of compressible boundary layers is essentially an eigenvalue problem described by the complex dispersion relation

$$
\alpha=\alpha(\beta, \omega)
$$

Since this code solves for spatial, not temporal, stability, $\beta$ and $\omega$ are assumed to be real, while $\alpha$ is complex. As is generally found in the study of system stability, the imaginary part of $\alpha$ represents the growth rate of boundary layer disturbances. In this case, $\alpha<0$ implies disturbance growth and instability within the boundary layer.

Malik has investigated both finite-difference and Chebyshev spectral methods to provide solutions for the above equations. The method used in this code for solving the eigenvalue problem, briefly outlined here, is discussed in greater detail in a 1990 paper by Malik [29].

Two different methods are used in this code to provide the eigenvalues and eigenfunctions. First, the system of equations is discretized using a staggered finite difference scheme. Then, using the QR algorithm, the spatial eigenvalues $\alpha$ are calculated. The eigenvalue solution is now refined using the following procedure. By using the continuity equation, the normal momentum equation is reduced in order from second to first. This transforms the five coupled second order linear stability equations into eight first-order equations given below. 


$$
\frac{d \Psi_{i}}{d y}-\sum_{j=1}^{8} a_{i j} \Phi_{j}=0 ; i=1,2, \ldots, 8
$$

where $\Phi_{1}=\hat{u}, \Phi_{2}=\frac{d \hat{u}}{d y}, \Phi_{3}=\hat{v}, \Phi_{4}=\hat{p}, \Phi_{5}=\hat{T}, \Phi_{6}=\frac{d \hat{T}}{d y}, \Phi_{7}=\hat{w}, \Phi_{8}=\frac{d \hat{w}}{d y}$, with the boundary conditions given below

$$
\begin{gathered}
\Phi_{1}=\Phi_{3}=\Phi_{5}=\Phi_{7}=0 ; y=0, \\
\Phi_{1}, \Phi_{3}, \Phi_{5}, \Phi_{7} \longrightarrow 0 ; y \longrightarrow \infty .
\end{gathered}
$$

A fourth-order accurate compact difference scheme for arbitrarily stretched grids [30] is used to solve for $\Phi_{i}$. Finally, employing a third-order accurate Newton's method, the final eigenvalues and eigenvectors are obtained. [30]

\subsubsection{Stability Calculation Results}

Results of the stability calculations are shown here for spatial amplification rates corresponding to boundary-layer disturbances ranging from $25 \mathrm{kHz}$ to $65 \mathrm{kHz}$ in increments of $5 \mathrm{kHz}$. Rates are only shown in this area since spatial amplification rates are not computed by the code $e^{\text {Malik }}$ for any disturbances outside of this frequency range. In the code, there are both global and local calculations performed. First the global calculations are performed. After the eigenvalue has met a global criterion set in the code, the stability computations switch to employing local values. If then the imaginary portion of the spatial amplification factor becomes negative, the disturbances begin to grow and an instability in the boundary-layer is produced. As these disturbances pass downstream along the blade surface, the cumulative amplification of this disturbance is quantified by computing an $\mathrm{N}$ number. Therefore, by studying the magnitude of the imaginary portion of $\alpha$, the spatial amplification rate, the "amount" of stability or instability in a system can be evaluated.

Figure 3.11 shows a comparison of spatial amplification rates for a boundary-layer disturbance with a frequency of $25 \mathrm{kHz}$. As was expected, the adiabatic wall case fell in the center of the heated wall and cooled wall cases in terms of stability, with 
the heated wall case being the least stable and the cooled wall case exhibiting the greatest stability (except right at the leading edge, where the trends are reversed). Another trend of note is the generally decreasing stability as you move downstream in the boundary-layer flow. Also of note is the fact that the $\alpha$ began being computed near the crown of the blade surface. This is consistent with the fact that the pressure gradient parameter starts to drop for a second time at this point, Fig. 3.7.

The spatial amplification rates for frequency disturbances between $30 \mathrm{kHz}-45$ $\mathrm{kHz}$ have the same general characteristics as the plot described above, Fig. 3.12 3.15. However, as $\beta$ grows, $\alpha$ is computed, for the most part, further back along the blade surface.

When a disturbance frequency of $50 \mathrm{kHz}$ is reached (Fig. 3.16), the more stable, cooled wall case only has two small areas where the global criterion is passed. The adiabatic case and the heated wall case results are still similar to the $\alpha$ results from the disturbances in the $30-45 \mathrm{kHz}$ range.

By a frequency of $55 \mathrm{kHz}$, the cooled wall case has failed to produce any $\alpha$ and the adiabatic wall case has now been split into two parts, Fig. 3.17. Also, after the minimum $\alpha$ was reached, the amplification rate grows much faster than for the lower frequency disturbances.

By the time a disturbance frequency of $60 \mathrm{kHz}$ is reached (Fig. 3.18), the adiabatic wall $\alpha$ has almost disappeared, with only a small area far back on the blade passing the global constraint. Also, the $\alpha$ value for the heated wall case begins climbing sharply immediately following the minimum value.

Local $\alpha$ 's are only being calculated for the heated wall case by the time a disturbance frequency of $65 \mathrm{kHz}$ is reached, Fig. 3.19. This again supports the theory stating that wall heating destabilizes wall boundary-layer flows. At $70 \mathrm{kHz}$ all of the cases fail the global test.

Figure 3.20 shows the effects of increasing the disturbance frequency on the stability of the baseline adiabatic wall case. Here, it can be easily seen that as the disturbance frequency increases, the region of least stability in the boundary is shifted downstream along the blade suction surface. Also, the frequencies centered about 40 $\mathrm{kHz}$ affect the most blade surface. 
To summarize, little instability and no transition was predicted on the entire blade suction surface up to the TE shock impingement.

\subsection{Free Stream Turbulence Effects}

Two important factors which affect the onset and length of boundary layer transition on gas turbine airfoils are the pressure gradient and the free stream turbulence characteristics. Free stream turbulence generally has the effect of hastening transition. For favorable pressure gradients, however, the flow acceleration acts to stabilize the boundary layer and tends to counteract the effect of free-stream turbulence. The interplay of a favorable pressure gradient and FST can influence both the onset and the length of transition. This complex interaction can be seen in Fig. 3.21 [31].

According to the figure, any FST level below 1\% should not produce transition on the turbine blade, since the Reynolds number based on momentum thickness does not go much higher than 400 anywhere on the blade suction surface. However, for FST levels over $2 \%$, transition should be achieved over some portion of the blade surface.

Using a grid designed by Johnson [32] to produce FST levels of $5 \%$, shadowgraphs were again taken to determine the state of the boundary layer under the influence of high levels of FST. However, it was found that the boundary layer remained laminar despite the high levels of free-stream turbulence. Upon closer evaluation of the turbulence characteristics, the length scales were found to be orders of magnitude larger than the boundary layer. It was postulated that since this FST was viewed more like a type of large scale flow unsteadiness than true free-stream turbulence due to its large length scale.

\subsubsection{Grid Design}

Since in a real engine environment the boundary layer on a substantial portion of the blade suction surface will be turbulent, it was necessary that a mechanism to "trip" the laminar boundary on our turbine blade be found. Using the stability calculations as a guide, D. Holmberg designed two wire mesh grids. The results of stability analysis 
given above were used to determine the frequency content and length scales of FST that would most likely excite the boundary layer and induce transition on the blade suction surface. It was decided that, given the inlet Mach number of 0.36 , FST with length scales on the order of $1 \mathrm{~mm}$ (0.0394 in) would produce FST with the proper frequency content to excite the boundary layer at the target frequency of $45 \mathrm{kHz}$.

The first of these grids (Grid 2) was constructed of 6 mesh wire mesh. The 6 mesh designation means that there are 6 spaces per inch, resulting in 11 wires across the $5.08 \mathrm{~cm}(2 \mathrm{in}) \mathrm{span}$ of the test section. The individual wires in the mesh posses a circular cross-section with a $0.889 \mathrm{~mm}(0.035 \mathrm{in})$ diameter. The turbulence intensity development is given by Eq. 3.40 and the length scale development is given by Eq. 3.41 assuming isotropic turbulence.

$$
\begin{aligned}
& T_{u}=1.07\left(\frac{x}{d}\right)^{-0.88} \\
& L_{x}=0.2 d\left(\frac{x}{d}\right)^{0.48}
\end{aligned}
$$

The second grid, Grid 4, was constructed with $\frac{3}{4}$ in mesh. This means there is a wire every $1.905 \mathrm{~cm}(0.75 \mathrm{in})$, resulting in 2 wires across the span. The individual wires in the mesh posses a circular cross-section with a $3.048 \mathrm{~mm}$ (0.12 in) diameter. The turbulence intensity development is given by Eq. 3.42 and the length scale development is given by Eq. 3.43 assuming isotropic turbulence.

$$
\begin{aligned}
T_{u} & =0.75\left(\frac{x}{d}\right)^{-0.73} \\
L_{x} & =0.29 d\left(\frac{x}{d}\right)^{0.3}
\end{aligned}
$$

In order to see the effect of varying the turbulence intensity of the FST, the two grids were each placed in two locations, for a total of four different values of turbulence intensity. The four locations and the resulting length and turbulence intensities are given in Table 3.3. Note that for Grid 2, the values in parenthesis are estimated values for non-isotropic turbulence. 


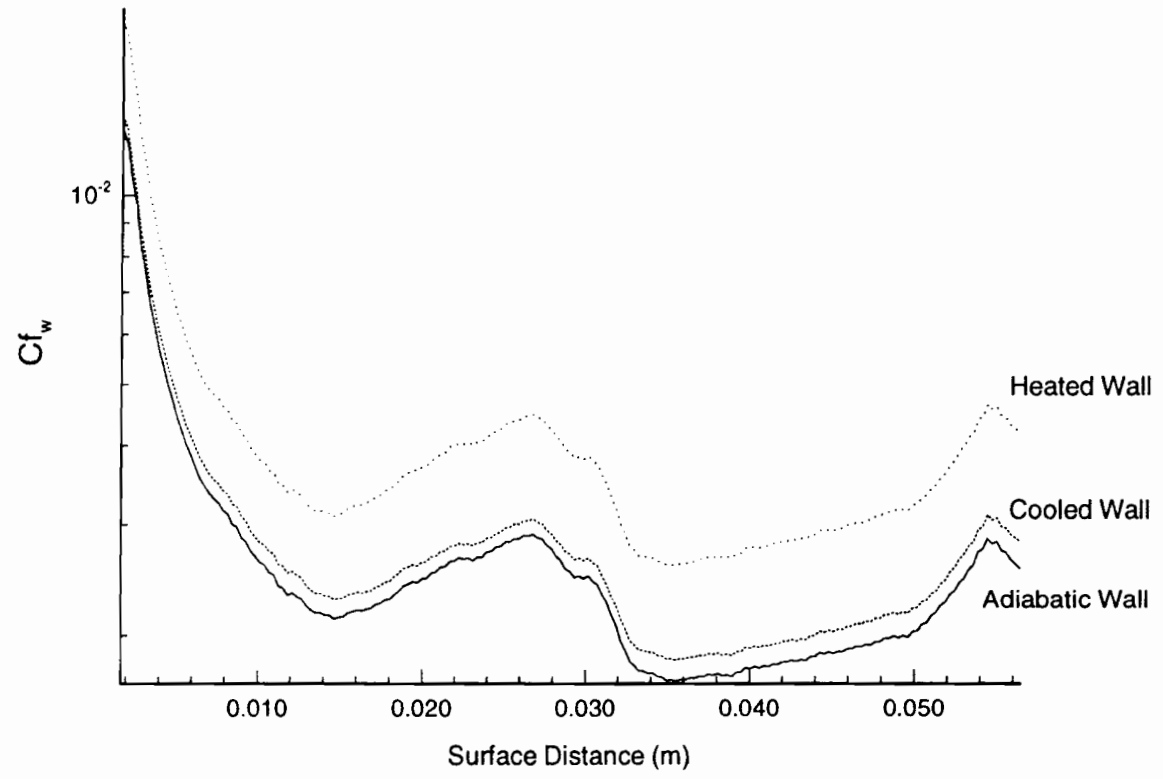

Figure 3.10: Steady Wall Skin Friction Distribution - All Cases

Table 3.3: Turbulence Grid Characteristics

\begin{tabular}{|c|c||c|c|}
\hline Grid \# & Dist. Upstream $(\mathrm{cm})$ & $T_{u}(\%)$ & $L_{x}(\mathrm{~mm})$ \\
\hline \hline 2 & 3.56 & 4.16 & 1.04 \\
\hline 2 & 1.27 & $10.3(9.0)$ & $0.637(0.8)$ \\
\hline 4 & 14.22 & 4.54 & 2.80 \\
\hline 4 & 3.56 & 12.5 & 1.85 \\
\hline
\end{tabular}




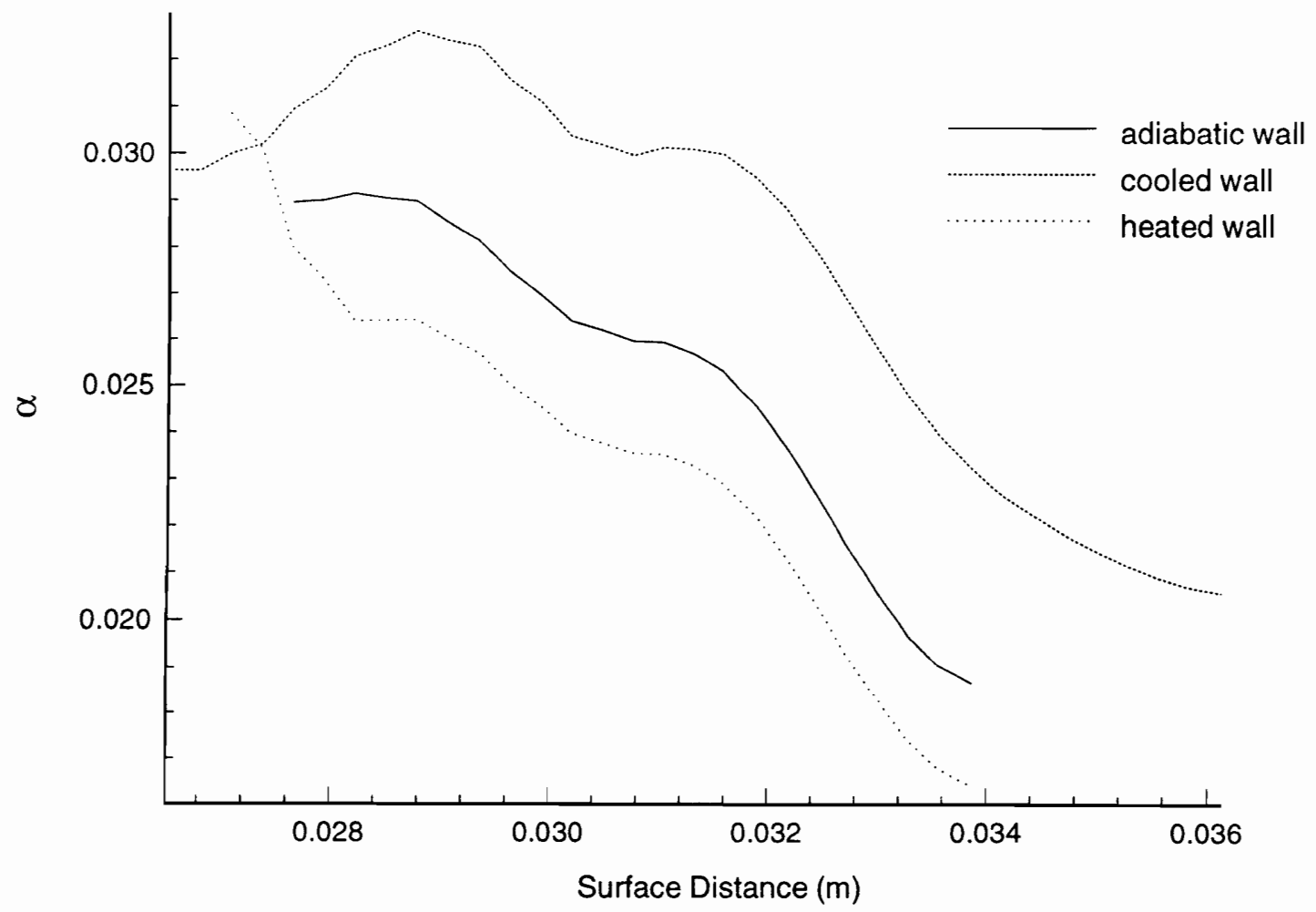

Figure 3.11: Comparison of Spatial Amplification Rates for a Boundary Layer Disturbance of $25 \mathrm{kHz}$ (Note negative $\alpha$ implies amplification) 


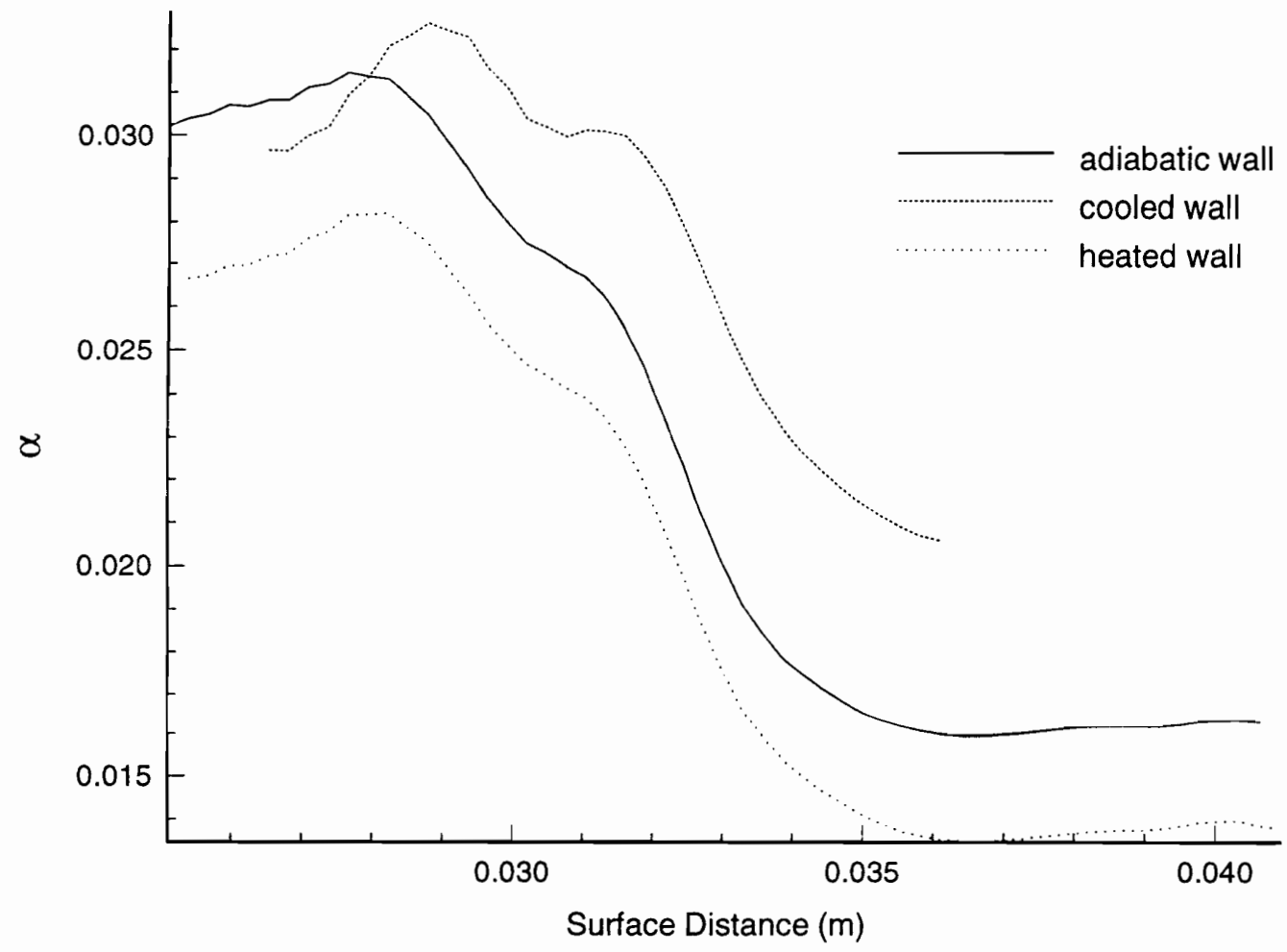

Figure 3.12: Comparison of Spatial Amplification Rates for a Boundary Layer Disturbance of $30 \mathrm{kHz}$ (Note negative $\alpha$ implies amplification) 


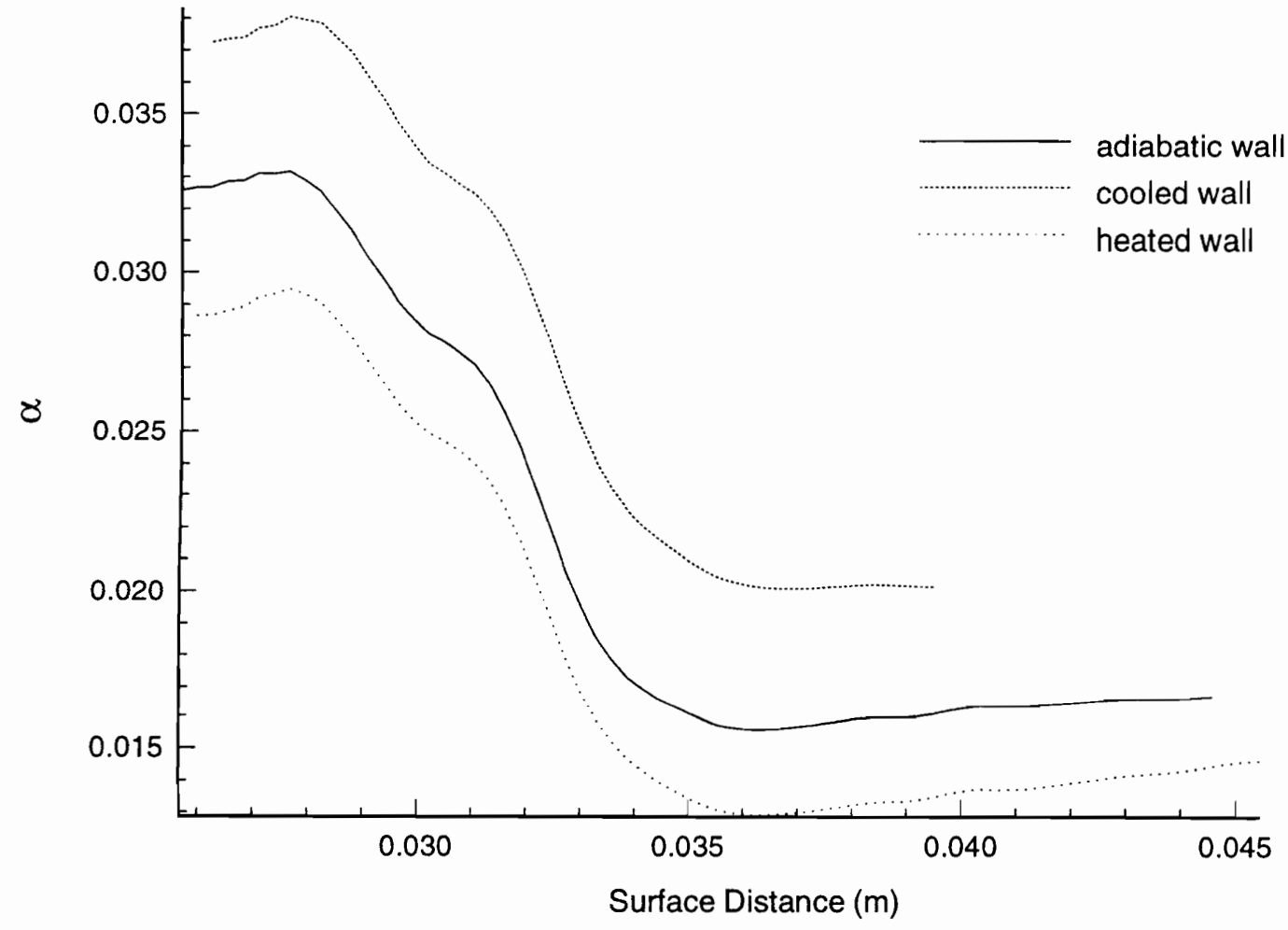

Figure 3.13: Comparison of Spatial Amplification Rates for a Boundary Layer Disturbance of $35 \mathrm{kHz}$ (Note negative $\alpha$ implies amplification) 


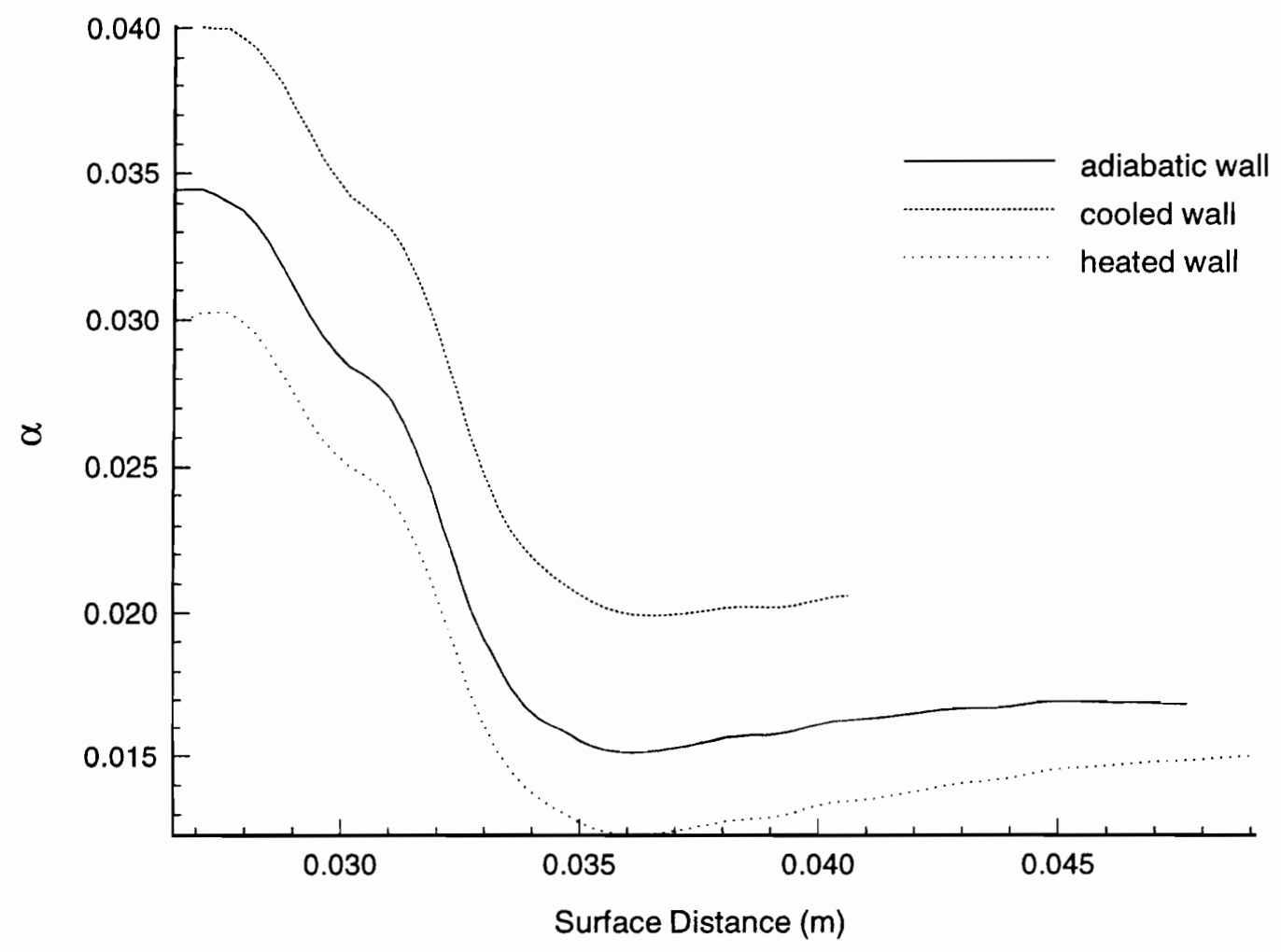

Figure 3.14: Comparison of Spatial Amplification Rates for a Boundary Layer Disturbance of $40 \mathrm{kHz}$ (Note negative $\alpha$ implies amplification) 


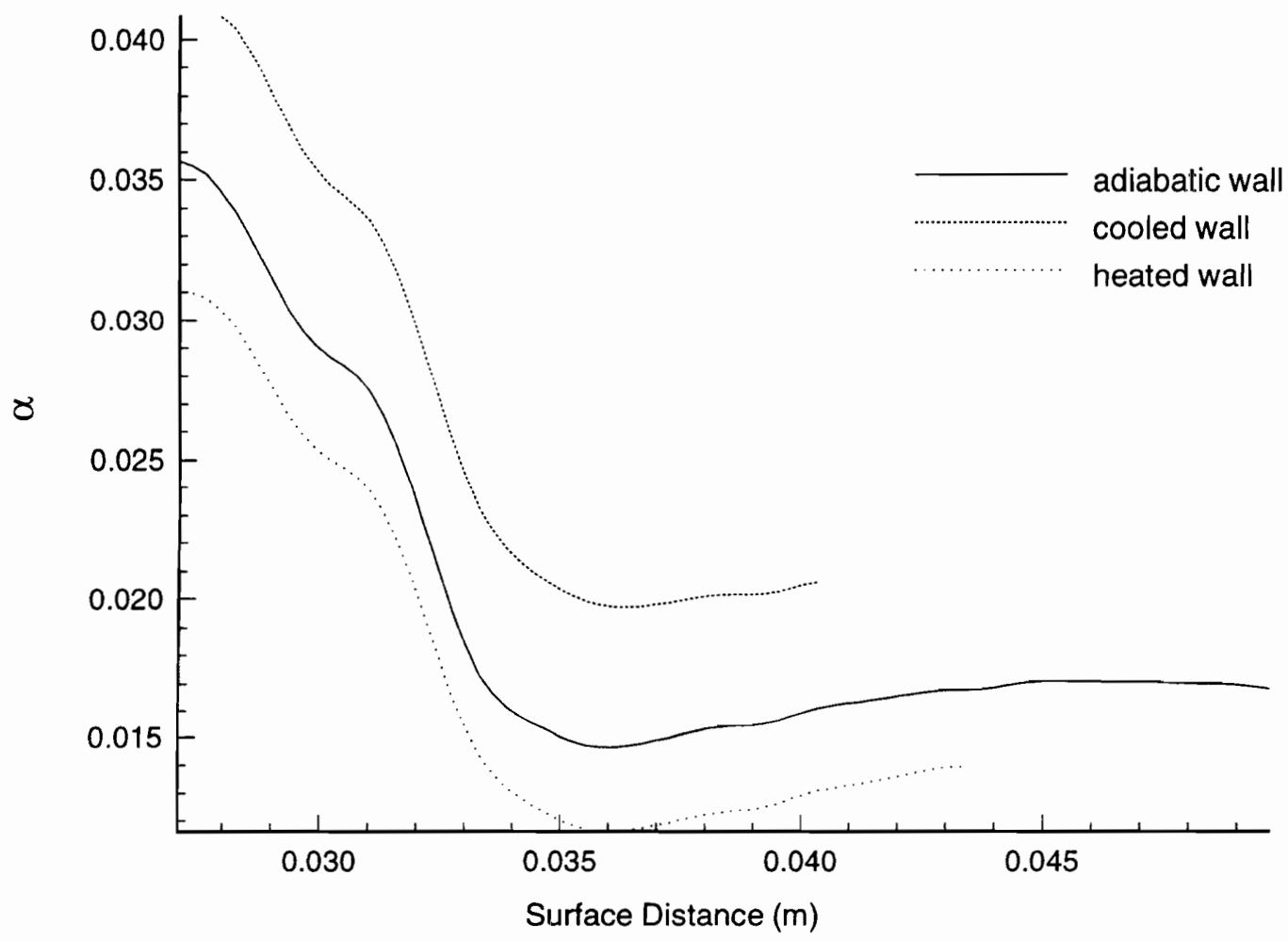

Figure 3.15: Comparison of Spatial Amplification Rates for a Boundary Layer Disturbance of $45 \mathrm{kHz}$ (Note negative $\alpha$ implies amplification) 


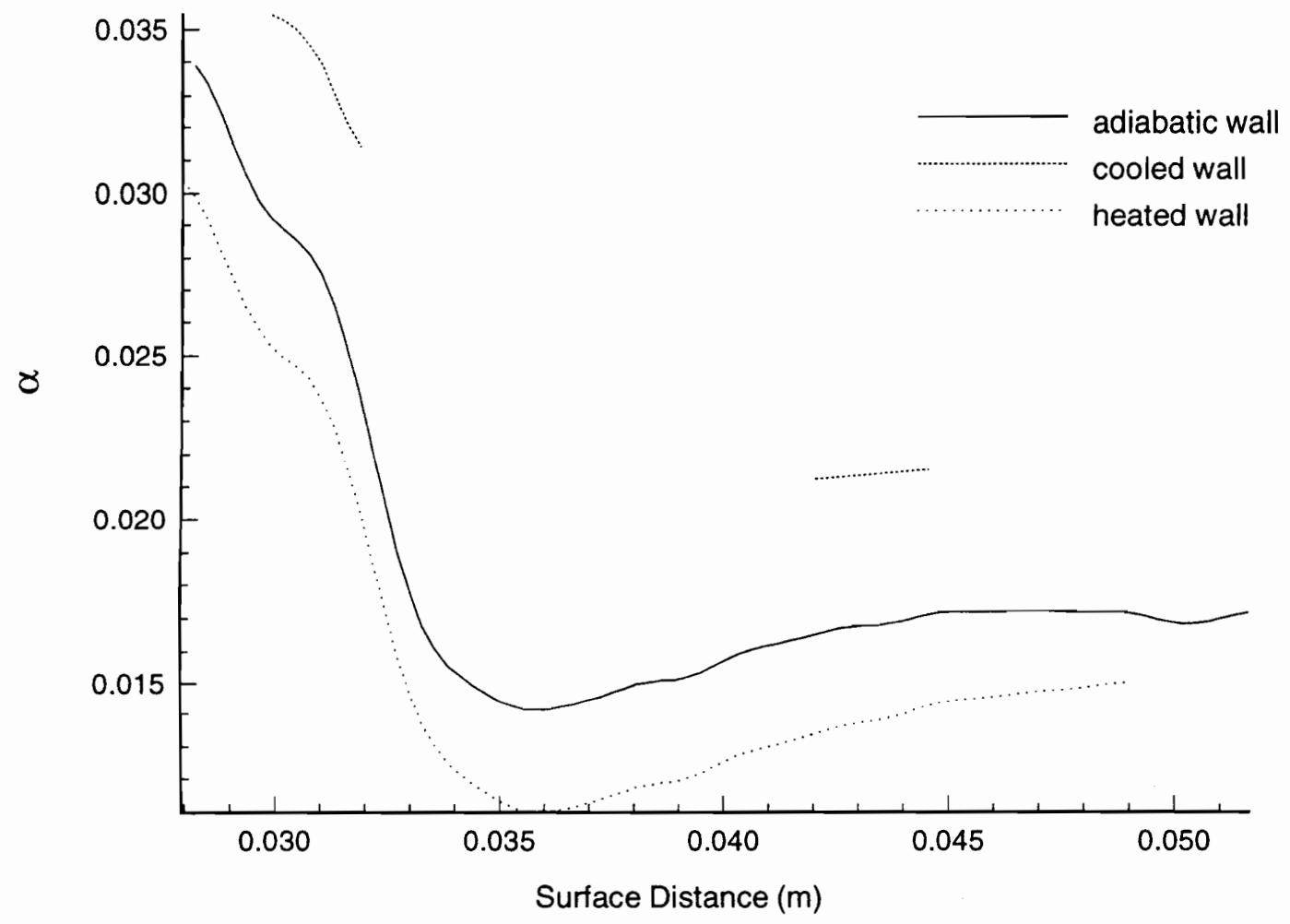

Figure 3.16: Comparison of Spatial Amplification Rates for a Boundary Layer Disturbance of $50 \mathrm{kHz}$ (Note negative $\alpha$ implies amplification) 


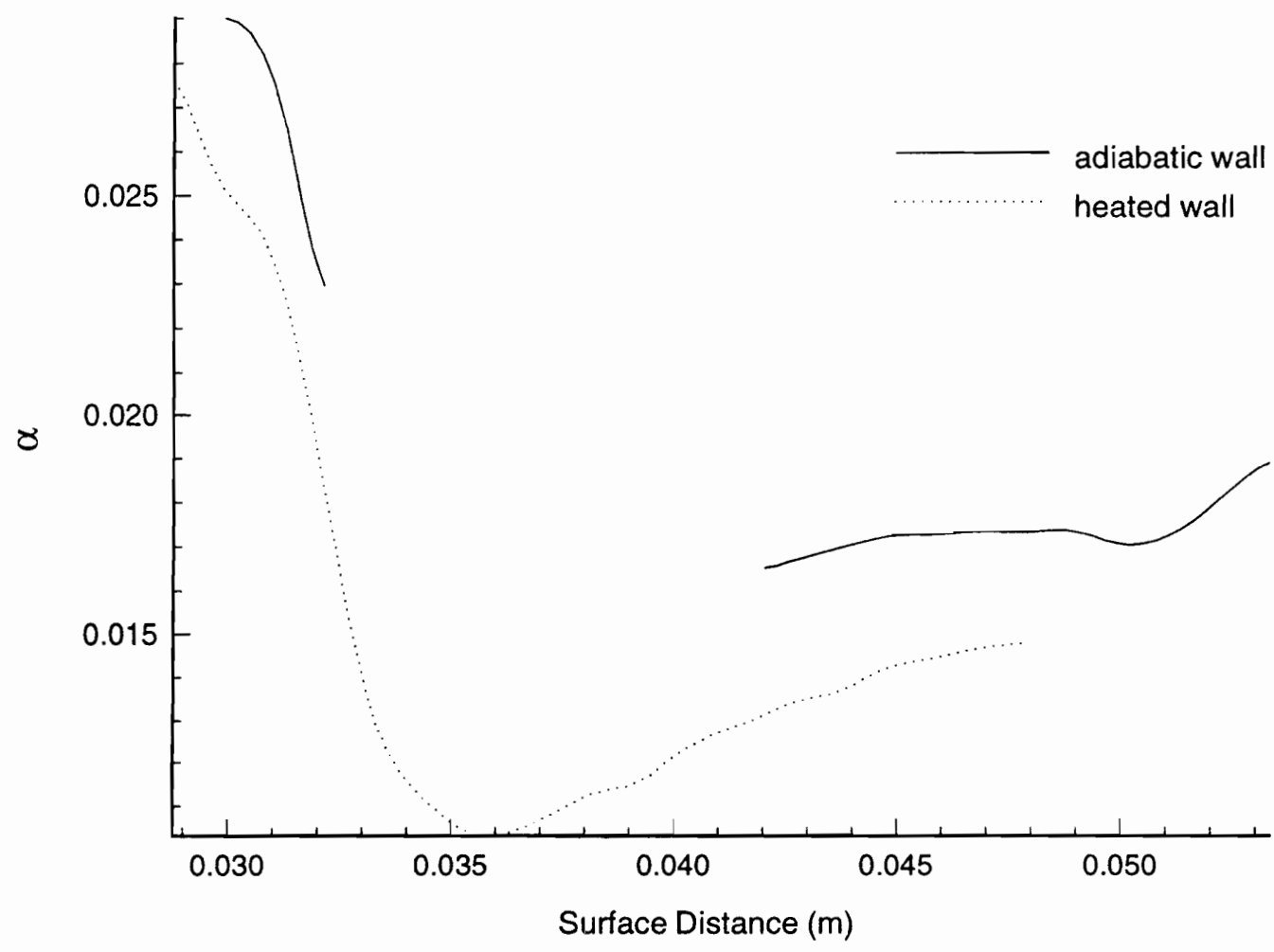

Figure 3.17: Comparison of Spatial Amplification Rates for a Boundary Layer Disturbance of $55 \mathrm{kHz}$ (Note negative $\alpha$ implies amplification) 


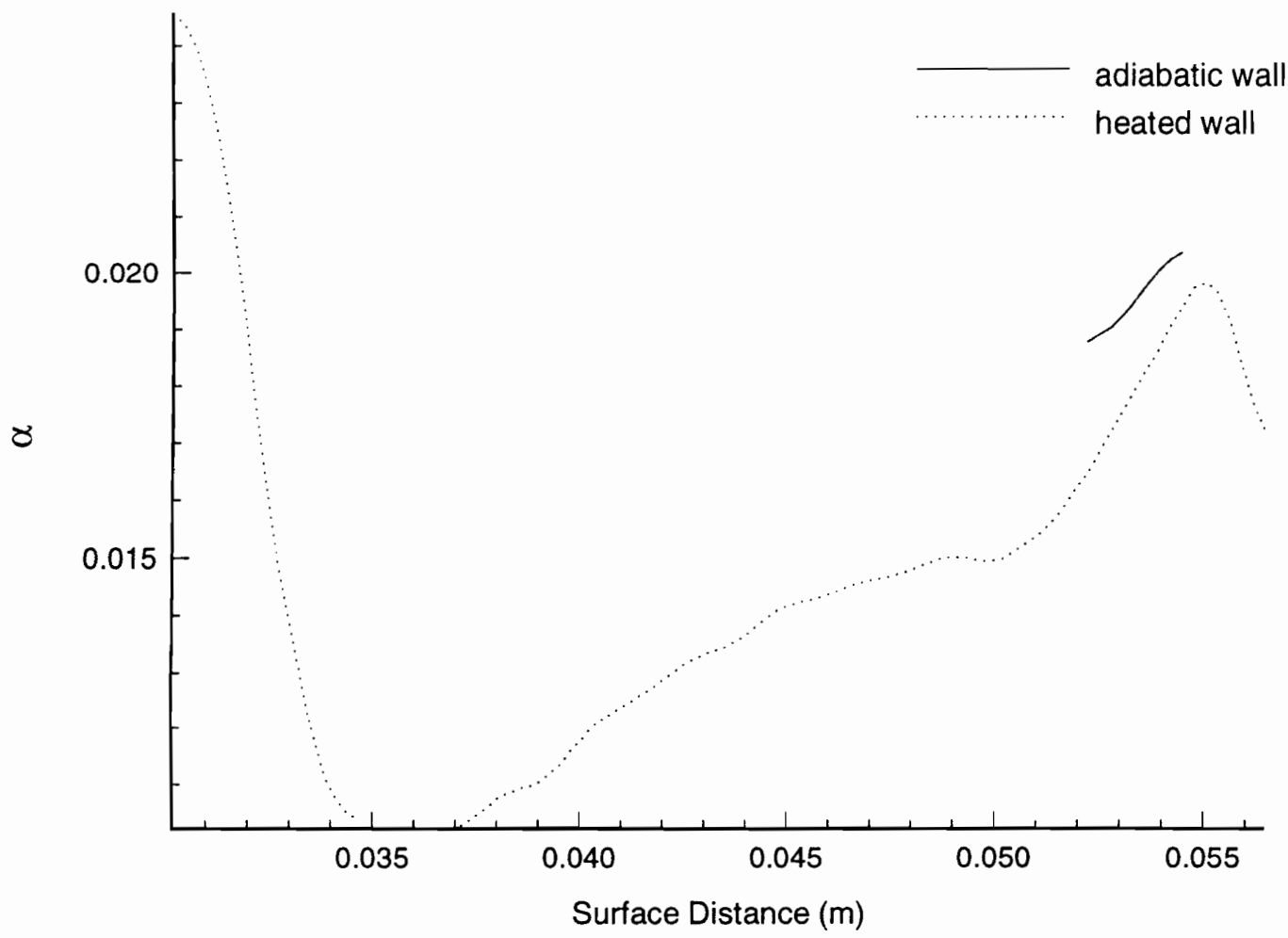

Figure 3.18: Comparison of Spatial Amplification Rates for a Boundary Layer Disturbance of $60 \mathrm{kHz}$ (Note negative $\alpha$ implies amplification) 


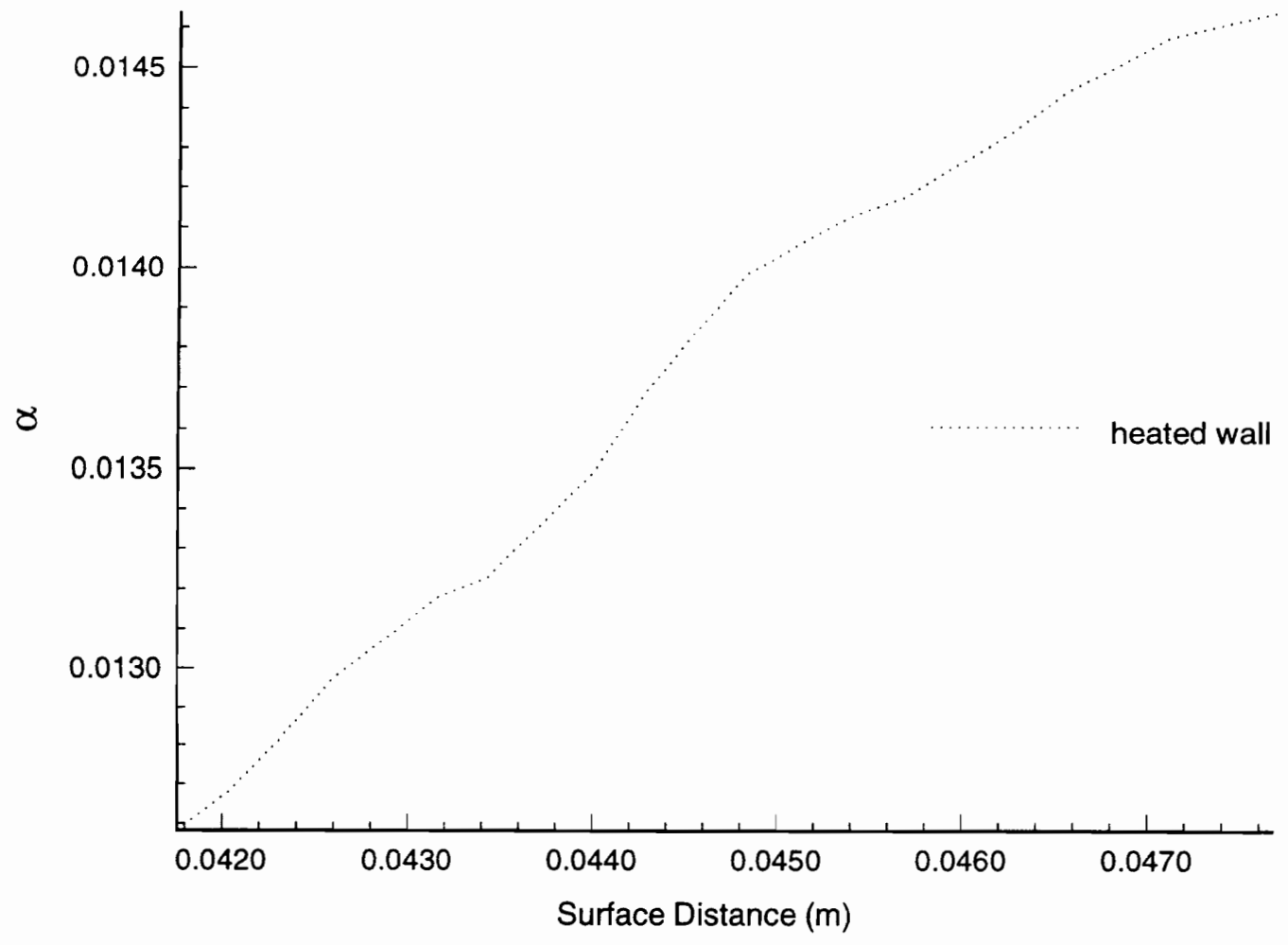

Figure 3.19: Comparison of Spatial Amplification Rates for a Boundary Layer Disturbance of $65 \mathrm{kHz}$ (Note negative $\alpha$ implies amplification) 


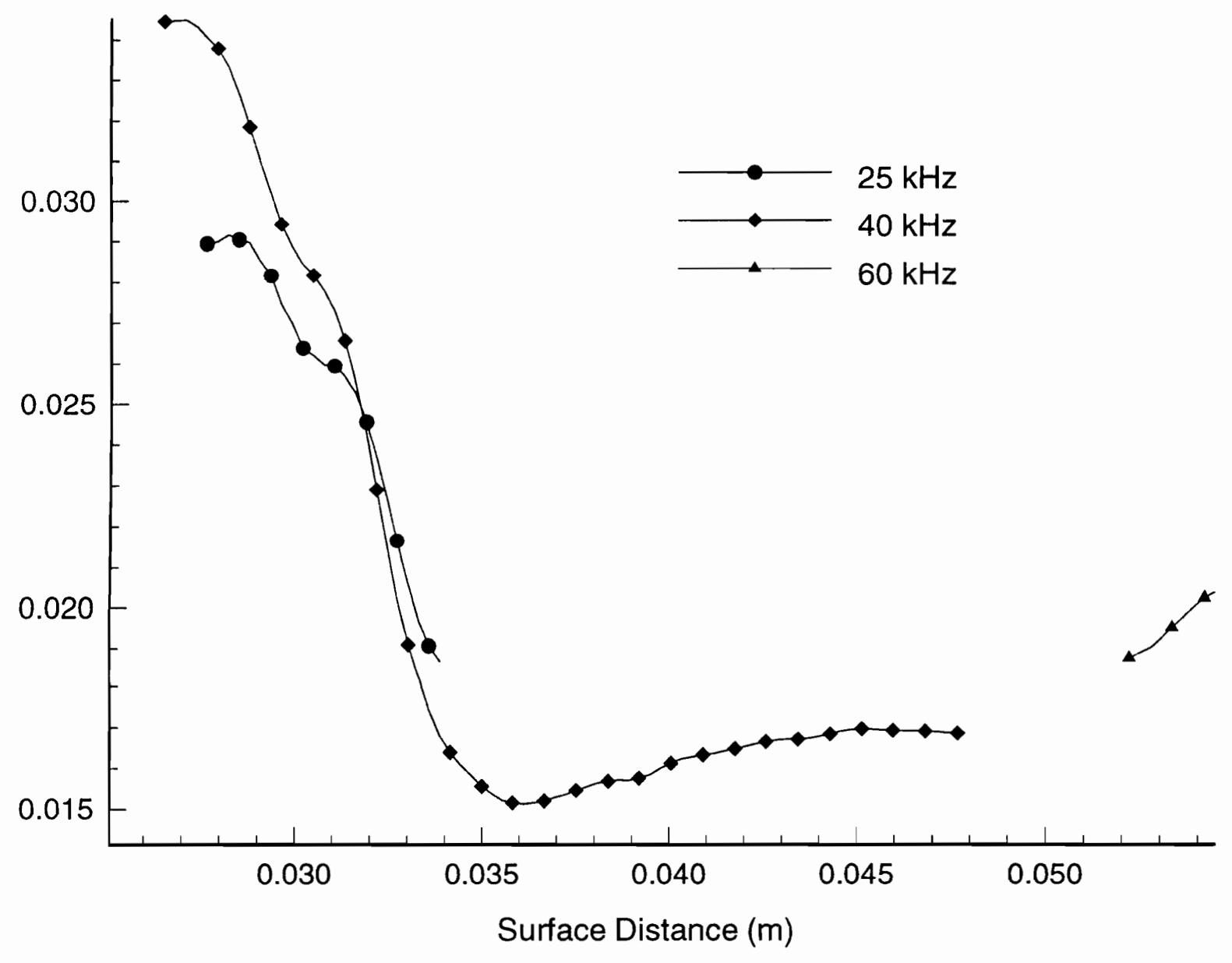

Figure 3.20: Comparison of Disturbance Frequency Effects on the Spatial Amplification Rates for the Adiabatic Wall Case (Note negative $\alpha$ implies amplification) 

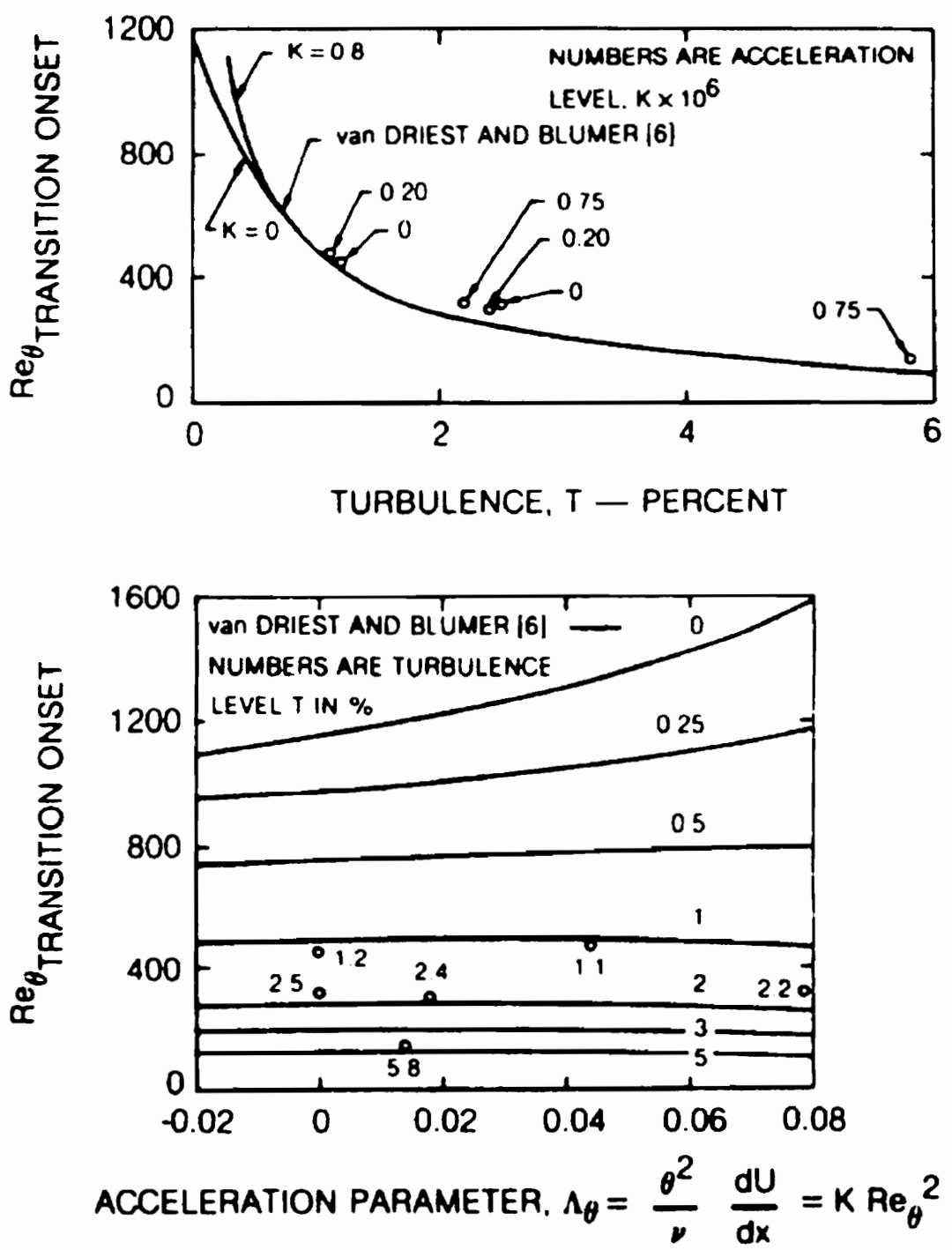

Figure 3.21: Combined Influence of Streamwise Pressure Gradient and Free Stream Turbulence Intensity on Boundary Layer Transition 
Another spark-shadowgraph study was undertaken to determine the FST influence on the state of the boundary layer.

\subsubsection{Shadowgraph Results with FST}

Figures $3.22-3.25$ show the effects of the varying free-stream turbulence levels and characteristics on the state of the boundary layer. In all of the shadowgraphs, instability waves and significant changes in the boundary layer profile over the laminar, low FST case are easily seen, indicating the presence of transition in all of the images. It is also noticeable that the effect of the FST turbulence varies depending on the grid type and location. Since it is difficult to pinpoint the exact onset of transition, these variations are difficult to quantify precisely. However, it is important to note that the stability analysis predicted the characteristics of the turbulence that the boundary layer would respond to, and only the grids designed specifically to produce FST with that characteristic frequency content produced transition on the blades. 


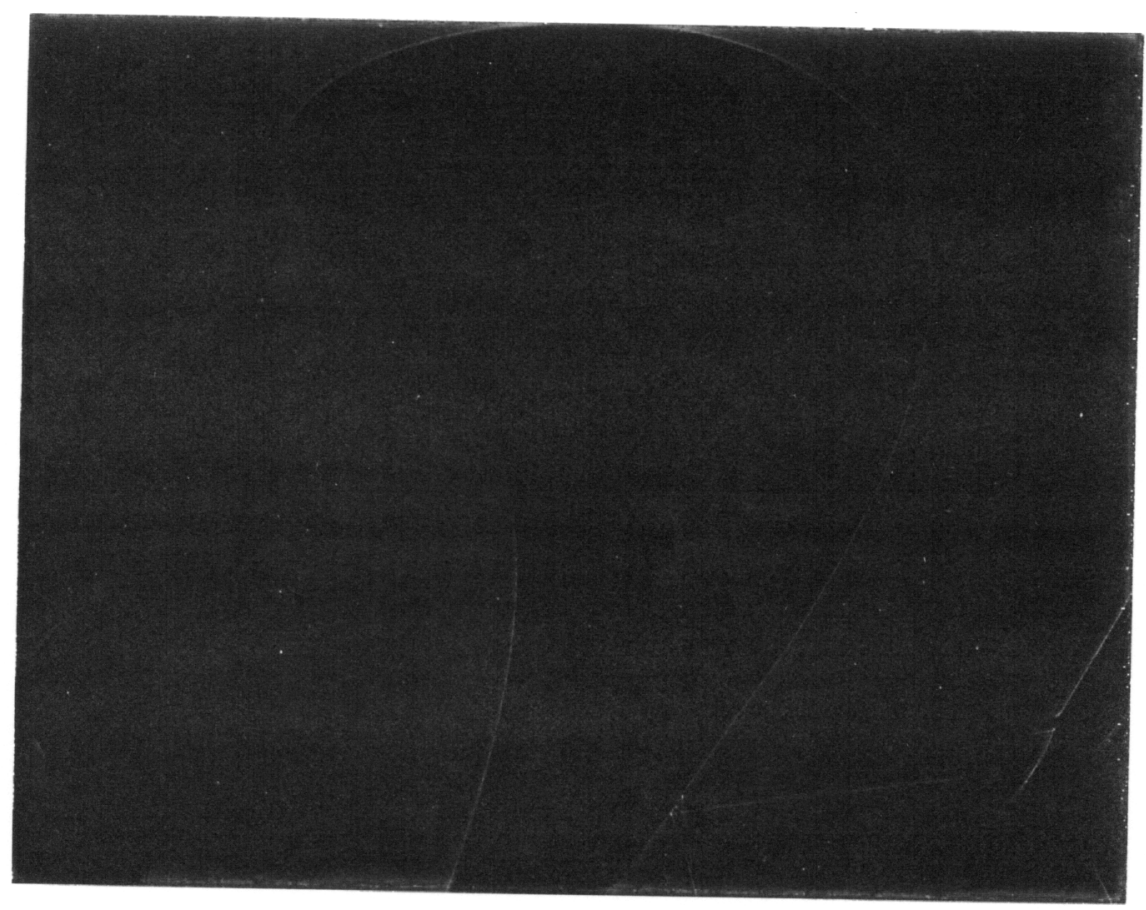

Figure 3.22: Magnified Spark-Shadowgraph of Boundary Layer Transition with Grid \#2 at $3.56 \mathrm{~cm}$ 


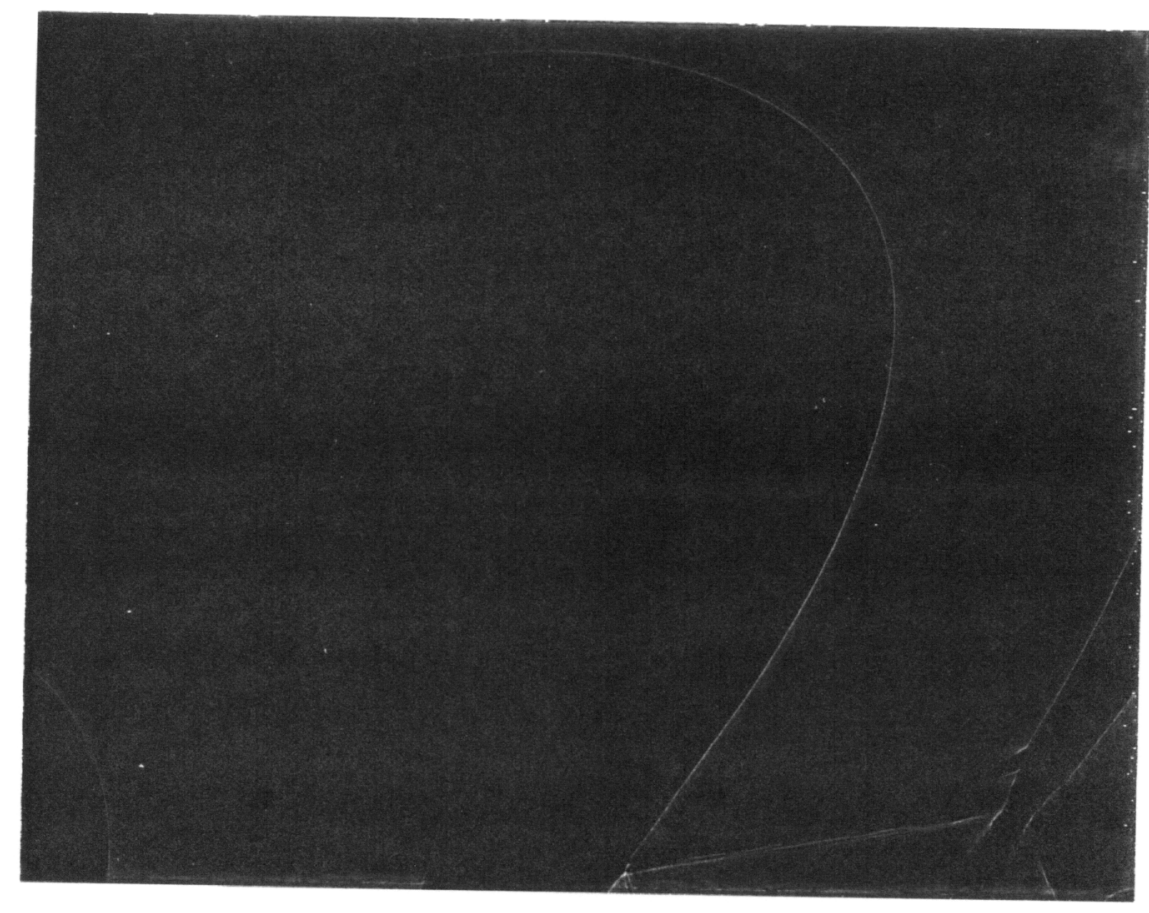

Figure 3.23: Magnified Spark-Shadowgraph of Boundary Layer Transition with Grid $\# 2$ at $1.27 \mathrm{~cm}$ 


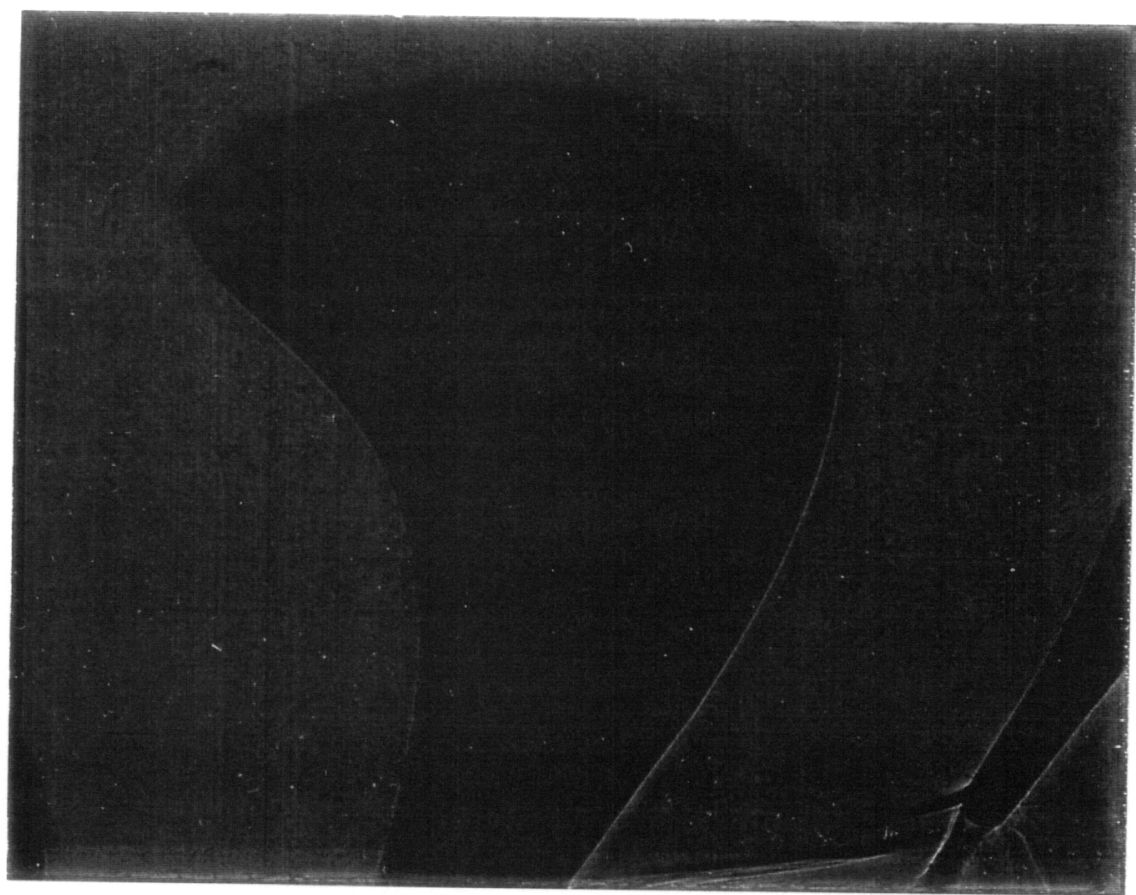

Higme 3.2.1: Magnified Spark-Shadowgraph of Bonmelary Laver Transition with (ivid \#1 at 1.2.2 (1) 


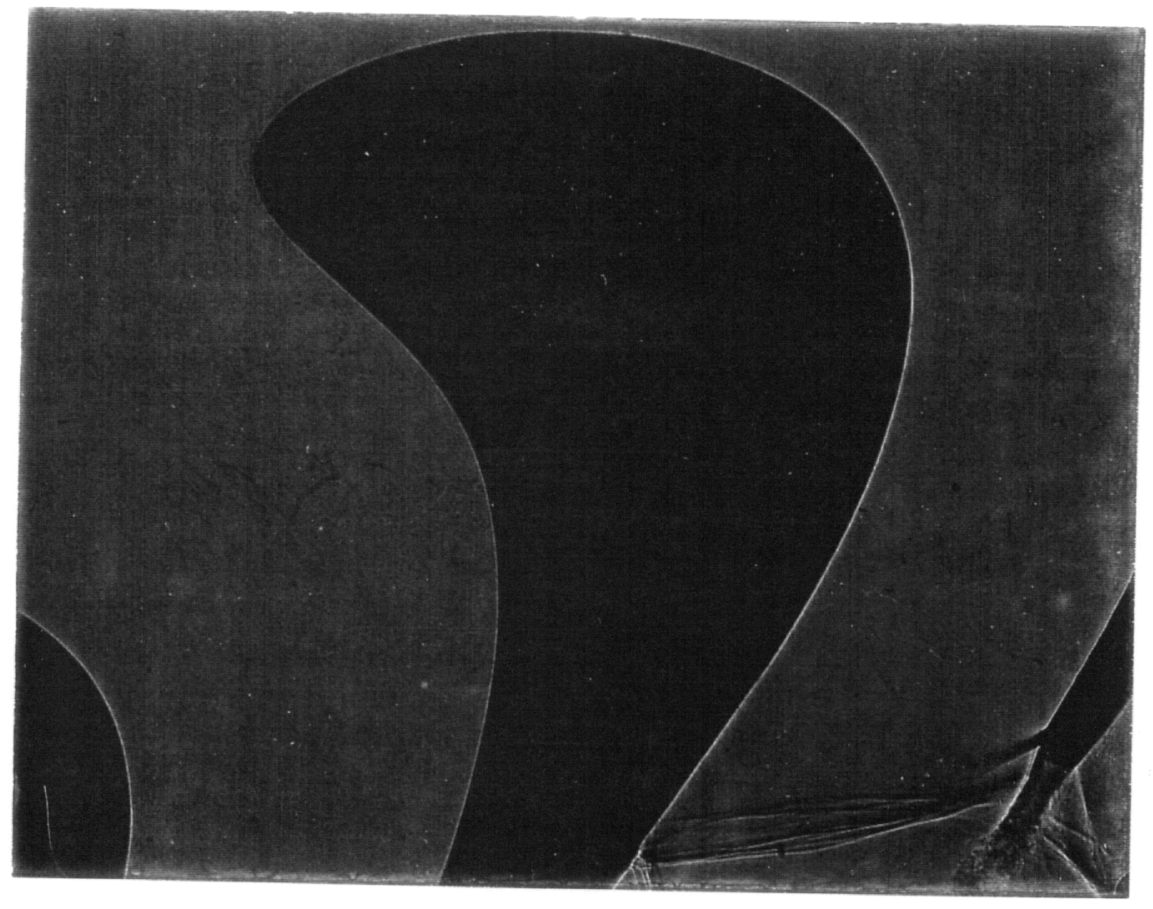

Figure 3.25: Magnified Spark-Shadowgraph of L mundary Layer Transition witl Crid \#4 a1 $3.560 \mathrm{~cm}$ 


\section{Chapter 4}

\section{Interferometric System Development}

\subsection{Introduction}

\subsubsection{Historical Background}

The basics of the reflection and refraction of light have been known since well before the 17th century. The interference of light, though not referred to as such until Young propounded his theories in the 19th century, had been observed independently by both Hooke and Boyle in 1665. Also in 1665, the phenomena of diffraction was observed by Grimaldi. The wave theory of light as first proposed by Hooke and then later expanded by Huygens explained all of these aspects of light rather neatly. Unfortunately, Huygens' theories had difficulty explaining the polarization of light. This shortcoming of Huygens' model allowed Newton's particle theory of light to hold sway over the scientific community for over a century.

In 1801, Young, in his classic double-slit experiment, showed that light from two sources can combine to form regions of brightness and darkness he called fringes. This phenomena, which Young termed constructive and destructive interference, respectively, could only be explained with the wave theory of light. Using this theory, a bright fringe would form where two waves combined in phase and a dark fringe would 
form where the two light waves found themselves $180^{\circ}$ out of phase. Young suggested that the only possible explanation was that light was a transverse wave.

The most dramatic advance in the understanding of light occurred in 1864 when Maxwell combined all of the experimental data from the work on electricity and magnetism conducted by Faraday, Oersted and Henry into a single set of equations. Using the velocity of light in a vacuum, Maxwell then showed that light was, in fact, an example of electromagnetic radiation made up a small region of the electromagnetic spectrum which stretched from long radio waves to cosmic rays. Unfortunately, despite the elegance of Maxwell's theory in uniting several different branches of physics, there were still problems with unexplained electromagnetic phenomena. While electromagnetic theory accounted for reflection, refraction, polarization, etc., it failed completely to explain emission and absorption.

With the discovery of the photoelectric effect in 1899 by Thomson, the theories on the nature of light were about to come full circle. In the photoelectric effect, light was proven to produce energy in discrete increments. Einstein concluded from these observations that electromagnetic radiation could only be described in the form of discrete particles, named photons. These apparent contradictions in the behavior of light have been termed the wave-particle duality. The wave theory accounts for effects like interference and diffraction, and the particle theory accounts for effects like blackbody radiation and the photoelectric effect. The measurement system developed here takes advantage of both the wave and the particle nature of light.

The modern theory of quantum electrodynamics (QED) has finally resolved these two apparently conflicting aspects of light and fully incorporated wave-particle duality into its theory. Here, all charged particles and photons are treated as quantized fields. QED is the theory of the interaction of charged leptons and photons, in which all observable effects can be expressed in terms of measured charge and mass [33].

\subsubsection{Fundamentals}

An optical interferometer is an instrument designed to exploit the interference of light and the fringe patterns that result from optical path differences. In order to achieve 
interference between two coherent beams of light, an interferometer divides an initial beam of light into two or more parts that travel different optical paths and then reunite to form an interference pattern. There are two basic types of interferometers: wavefront division interferometers and amplitude division interferometers. A wavefront division interferometer samples portions of the same wavefront of a coherent beam of light, as in Young's double slit experiment. An amplitude division interferometer instead uses some type of beam splitter that divides the beam into two parts. Single-plate interferometry is of the former type.

Interferometry is useful in the study of gas-flow problems for the same reasons that the shadowgraph and the schlieren apparatus are useful. Specifically, it permits observation of the flow without requiring the insertion of pressure probes or other measuring instruments into the flow that could cause disturbances in the flowfield. Also, it provides a photographic record that can be studied at leisure and permits rapid flow fluctuations to be "frozen". As opposed to the shadowgraph and schlieren methods, the interferometer provides quantitative, as well as qualitative, evaluation of the density field.

The most commonly used type of interferometer is the Mach-Zehnder type, developed in the 1890's by Mach and Zehnder for use in studying the phenomena of airflow and ballistics. Most of these interferometers are used for the study of supersonic flows. (See Refs. [24] and [34] for a general discussion of these instruments) Since the light source used in a Mach-Zehnder type interferometer is not coherent, it is necessary to adjust the path length difference between the reference and working beams to be less than one-tenth of a wavelength of the light, or $5 \times 10^{-6} \mathrm{~cm}$. Thus, this type of interferometer is very sensitive to temperature changes and mechanical vibrations. Large vibration damping equipment and a temperature controlled environment is, therefore, necessary for the use of this type of interferometric system.

However, with the development of the laser, a source of highly coherent light became available, and non-common path interferometric testing became possible. Single-plate interferometry takes advantage of this advancement in optics. Figure 4.1 shows the basic optical components of a single-plate interferometric system. A laser is required, instead of a conventional light source, due to the relatively large $2.54 \mathrm{~cm}$ 
(1 in) path length difference caused by reflections off the front and rear surfaces of the wedge plate. This large path length difference requires a light source with a long coherence length, and such large coherence lengths can only be produced by a laser light source.

Next, the light beam passes through a spatial filter assembly. This assembly not only conditions the beam, but also expands it onto the collimating parabolic mirror, producing the required beam diameter. The parallel beam then passes through the test section and onto the wedge plate. The wedge plate splits the light beam into two overlapping beams with a slight angle between them. It is the action of the wedge plate that causes fringe formation. The wedge plate is also the cause of the most distinct feature of single-plate interferograms, the presence of a "double image". Due to the light reflection off both the front and rear surfaces of the wedge plate, all objects in the test section create two separate images at the image plane. The distance between the two images is referred to as the image separation distance. The second parabolic mirror is used to focus the parallel light beams. This image can be focused onto a photographic plate, or directly into a CCD camera.

Some of the advantages of a single-plate interferometer over the more frequently employed Mach-Zehnder interferometer can now be easily seen. First of all, since the parallel beam is split only after it passes through the test section, no compensation chamber is needed. Also, since the wedge angle is fixed, there is no need for heavy vibration free mounting of the wedge plate. Furthermore, only a single wedge plate is needed, compared to the two beam splitters and two flat mirrors required for the Mach-Zehnder interferometer. Finally, and most importantly, the setup of a single-plate system is much easier than for the conventional Mach-Zehnder system. Disadvantages include lower fringe quality and more difficult data interpretation. For a single-plate interferogram a domain in the flowfield with a know density distribution will be required, however this flowfield can be chosen in a region where data acquisition is relatively easy. 


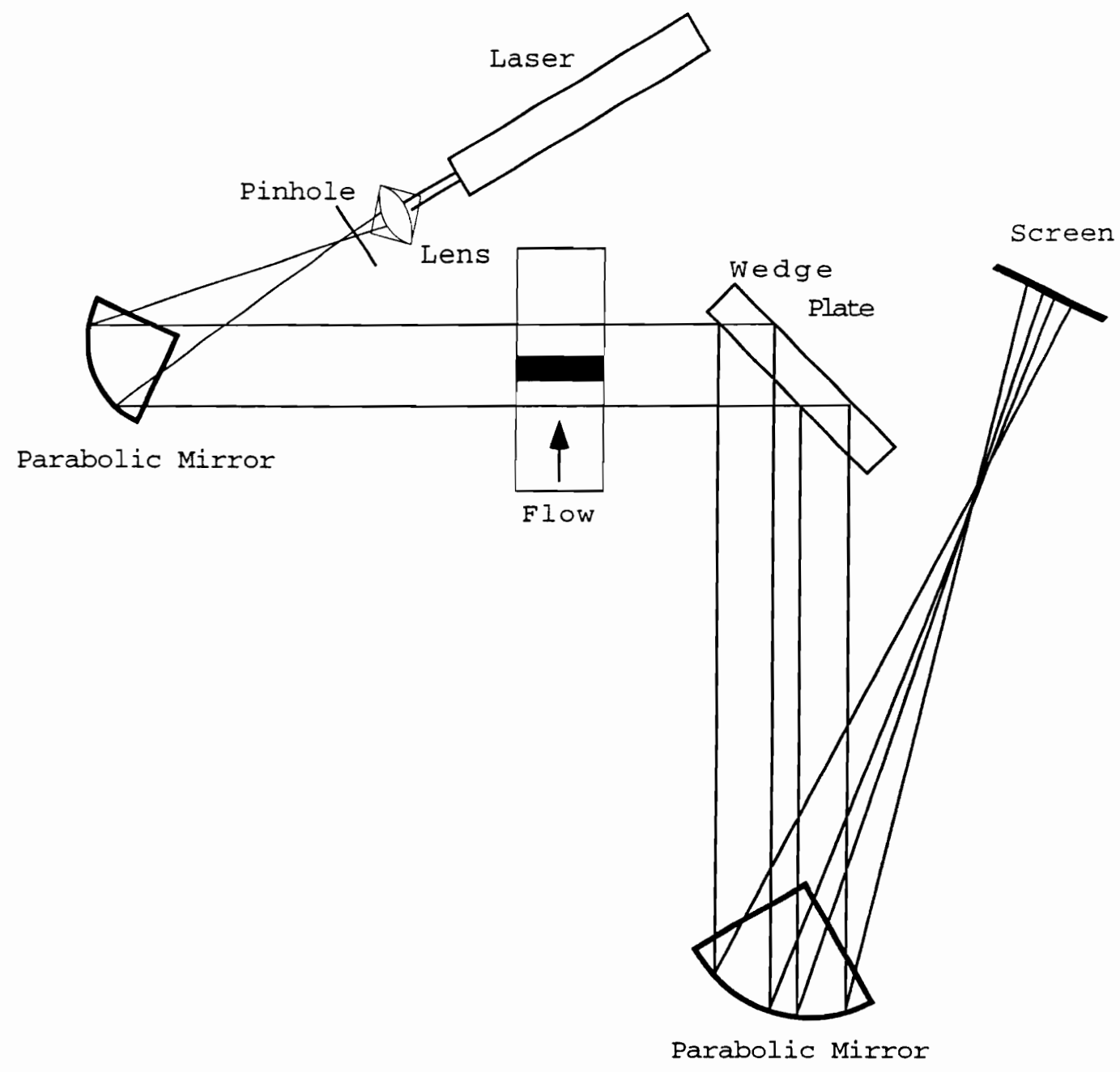

Figure 4.1: Basic Single-Plate Interferometric System 


\subsubsection{Interpretation}

Details of interpreting single-plate interferograms can be found in the dissertation of Kiss [7]. Here, only the basic equations and a simplified explanation of the fringe formation is presented.

Figure 4.2 depicts the image formation for a certain orientation of the wedge plate. If one picks two points, $A$ and $B$, in the interferogram, the $A_{1}, A_{2}, B_{1}$ and $B_{2}$ points can be traced back to the test section. Then, the following relationship can be written between the densities at these points:

$$
\left(\rho_{A_{1}}-\rho_{B_{1}}\right)-\left(\rho_{A_{2}}-\rho_{B_{2}}\right)=-\frac{\lambda_{0}}{K}\left(f_{A B_{f}}-f_{A B_{n}}\right)
$$

where $\lambda_{0}$ is the wavelength of the laser light, and $\mathrm{f}_{A B_{f}}$ and $\mathrm{f}_{A B_{n}}$ are the number of fringes between points $A$ and $B$ in the interferogram with flow and without, respectively. The $\mathrm{K}$ constant is given by

$$
K=\frac{L k}{n_{0} \rho_{r e f}}
$$

where $\mathrm{L}$ is the span of the test section, $\mathrm{n}_{0}$ is the index of refraction for ambient air, $\rho_{\text {ref }}$ is a reference density $\left(1.252 \mathrm{~kg} / \mathrm{m}^{3}\right)$ and $k=3 \times 10^{-4}$.

Looking at the Eq. 4.1, it is clear that if the absolute value of the density at a given point is to be found, the density has to be known at three other locations. It can further be shown that when the density is known in a band having the width of the image separation distance, $d$, then the density can be found anywhere else in the studied flowfield.

\subsection{Previous Steady Flow Interferometric System at Virginia Tech}

The steady interferometric system at Virginia Tech prior to this work deviated from the simple design in Fig. 4.1 due to the addition of a Bragg cell, a photo diode, and a complex time delay / triggering system. A $15 \mathrm{~mW}$ helium-neon (HeNe) laser was 


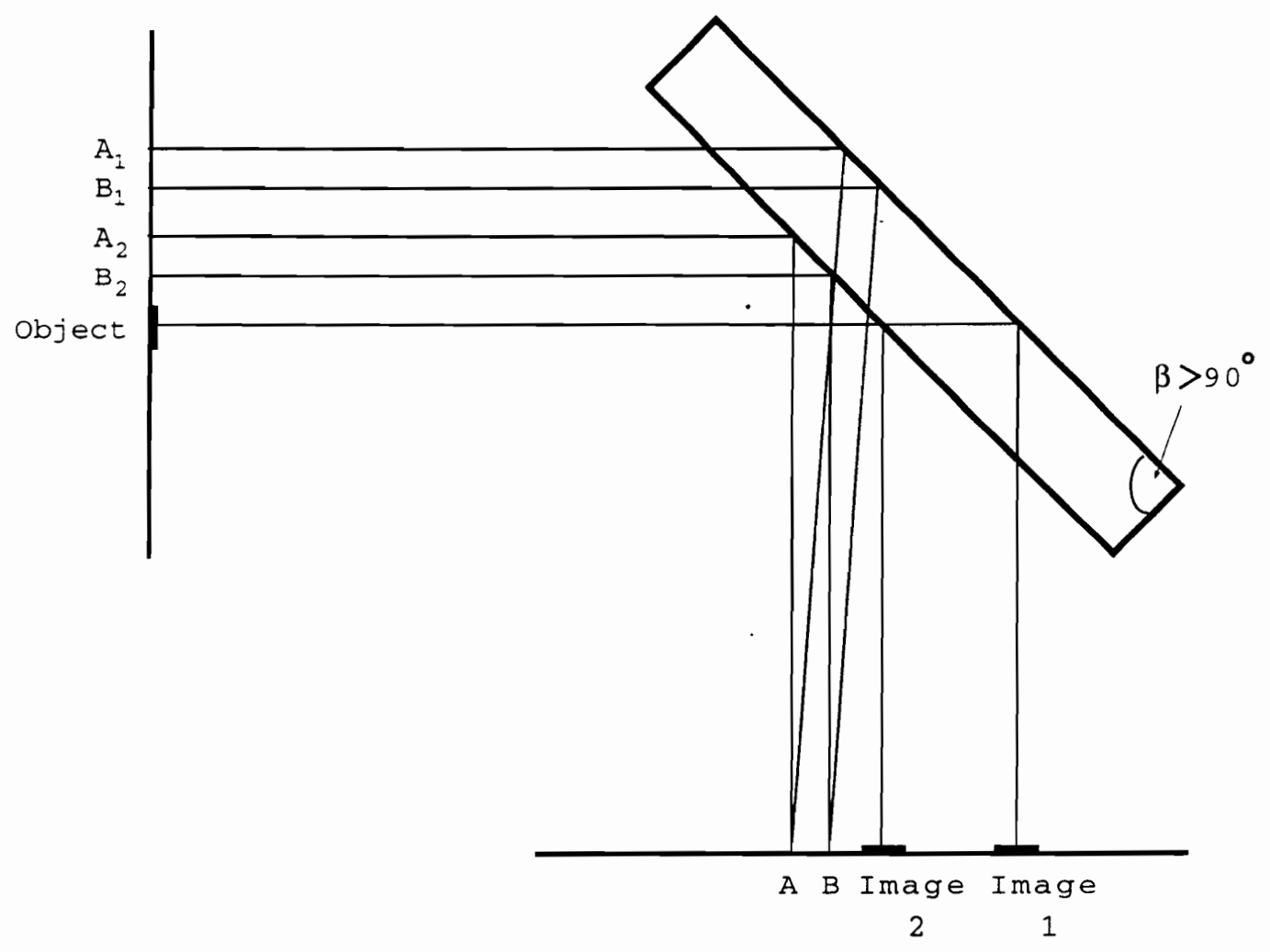

Figure 4.2: Light Path Through Single-Path 
used for the light source. A $32 \mathrm{~mm}$ focal length lens and $100 \mu \mathrm{m}$ pinhole were used in the spatial filter assembly, producing a $10.16 \mathrm{~cm}(4 \mathrm{in})$ diameter beam of collimated light. The wedge plate was a $15.24 \mathrm{~cm}$ (6 in) diameter, $0.5842 \mathrm{~cm}(0.23 \mathrm{in})$ thick plate. This plate nominally produces a $0.5334 \mathrm{~cm}(0.21 \mathrm{in})$ separation distance in the interferograms. The fringe spacing for no-flow conditions of $0.2286 \mathrm{~cm}(0.09 \mathrm{in})$ gives 15 seconds of arc wedge angle. Also, Polaroid (type 57) film, placed at the image plane, was the method of capturing the fringe pattern. An array of 9 static pressure taps were used to obtain the required known flowfield.

Because of the presence of higher frequency fluctuations in the "steady" flowfield, short $(100 \mu \mathrm{s})$ exposure times are required. Due to the prohibitive expense of pulse lasers, a readily available continuous HeNe laser was used as the light source. Thus, a very fast shuttering mechanism was needed. Mechanical shutters are only capable of operating in the millisecond range. Therefore, another method to shutter the light beam was devised. The final system is shown in Fig. 4.3 uses a Bragg-cell, driven by a very short square signal, to deflect the beam through the pinhole for the required 100 $\mu \mathrm{s}$. Some problems were encountered with this application, since the main undeflected beam bleeds light through the pinhole onto the exposed film, contaminating the image. Therefore, a mechanical shutter, placed directly after the laser, was used to block the unwanted, scattered light. The opening of the mechanical shutter was synchronized with the opening of the Bragg-cell through the use of a photodiode.

\subsection{Required System Improvements for Unsteady Flows}

In this section, some of the improvements that were required to produce viable unsteady interferograms are described. In the unsteady flowfield of interest here, the passing shock generated by the shock tube travels through the test section at a nominal velocity of $340 \frac{\mathrm{m}}{\mathrm{s}}$. This flow feature is the driving force behind many of the required changes.

First of all, much shorter shutter times, of the order of $0.33 \mu \mathrm{s}$, are needed to 


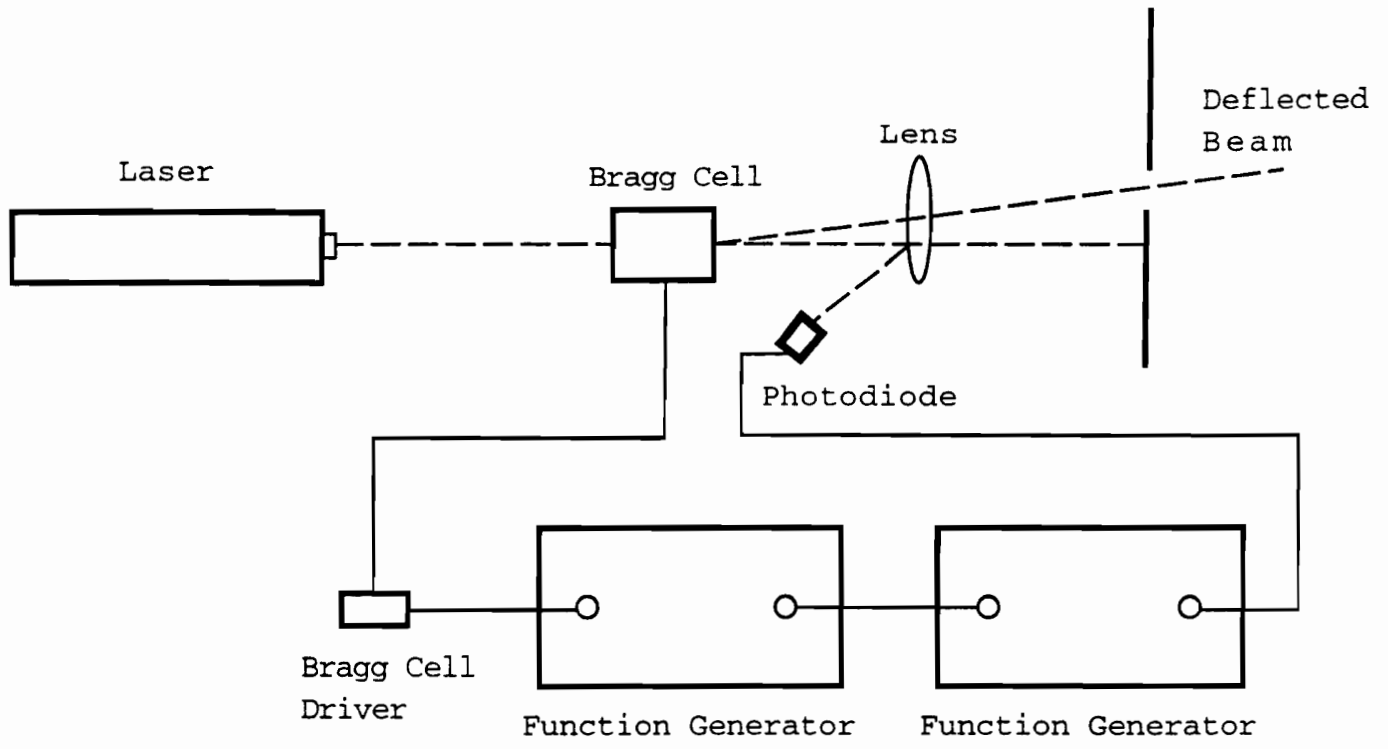

Figure 4.3: Bragg Cell Shutter System 
capture the image of the passing shock without excessive blur. The required shutter time was calculated by allowing the shock only $0.1 \mathrm{~mm}$ of motion during the image exposure time. However, creating such short shutter times is extremely difficult. The shorter shutter time required an increase in light intensity of at least two orders of magnitude at the photographic plane. With this increase in power, a Bragg-cell cannot withstand the high voltage required to create the desired shutter time. Also, even if it was possible to shutter the light with a Bragg-cell, the light bleed, with the addition of the mechanical shutter, would still contaminate the image. Another method of shuttering the light and a higher powered laser were required.

Another problem is synchronizing the shuttering of the light source with the passing of the shock. Previously, because the flowfield was steady, the shuttering sequence was triggered by hand. This method is not acceptable here, since the data collection and the interferograms must be taken during different runs. All influence from the shock impingement has left the studied blade row in a matter of microseconds.

Finally, the last major problem of collecting a known density field in these unsteady conditions needed to be addressed as well as the difficulty of referencing all the data together. Again it should be noted, that although a small portion of the density field is required to be known, it can be chosen in an area of the flow field that is easily accessible and where the measurement devices are least likely to disrupt the flow.

The first possibility that was investigated was simply switching to a pulsed laser system. Because of cost constraints, the purchase of a high powered pulse laser system was not acceptable. However, the Aerospace and Ocean Engineering Department of Virginia Tech had an extremely high powered, pulsed copper-vapor laser system. Unfortunately, because of the extremely high temperatures and ion velocities reached in the lasing cavity of a Cu-vapor laser, the light is fairly non-monocromatic. Nonmonocromatic light exhibits poor temporal coherence - a measure of the degree of phase correlation that exists in the radiation field of a light source at different locations and different times. In order to obtain the required coherence length of the laser light for this application, very careful filtering of the light would have been required. The addition of an etalon (reducing the light intensity to less than $5 \%$ of the original level) 
or pumping of a secondary laser with the copper vapor laser would have been necessary [35]. This fact made the system very difficult to apply in this tunnel environment, especially since ease of setup is one of the major general system advantages.

Next, the possibility of keeping the Bragg-cell shuttering method, and using a higher powered continuous laser to compensate for the light intensity reduction was investigated. It was decided to design the system with a 2-5 $\mathrm{W}$ argon gas laser in mind. Argon gas lasers of this power are fairly common in academia. Therefore, it was likely that one could be found "in house". Because of the high voltages needed to shutter the Bragg-cell at the required speed, using a cascade of two Bragg-cells was investigated. However, due to the large light power losses through the cell, the cascade was determined to be inappropriate.

Since the illumination of the photographic plate seemed to be the major stumbling block to the applicability of the system, ways to increase the light availability were investigated. Simply increasing the laser output power was not an option, since setting the laser to a higher output current destabilized the coherence length and the addition of an etalon would have dramatically reduced the power output. Kiss had similar laser power problems and had suggested the idea of coating the wedge plate to reduce the approximately $90 \%$ power loss through the plate. This solution was investigated, however, due to polarity effects, this was determined to be extremely difficult.

While researching various possibilities for increasing light intensity, a reference to intensified cameras - specifically, intensified CCD (Charge-Coupled Device) cameras was uncovered. CCD intensifier cameras typically have nanosecond shuttering times and high gain capabilities. The addition of the CCD camera offered many advantages and simplifications. The CCD camera allows the removal of the mechanical and Bragg-cell shutters and simplifies the triggering system. Also, with the presence of the intensifier, the use of even faster shutter times and the return to the use of the lower powered $\mathrm{HeNe}$ laser might be possible.

Next, the synchronization problem needed to be addressed. In order to produce a sequence of interferograms showing the passing of the shock, a triggering and synchronizing mechanism was sought. While performing preliminary testing on the shock 
system, a simple setup using a high frequency pressure transducer was employed to initiate the data collection. It was decided to use a modified version of this setup to initiate and synchronize all of the data collection. A high frequency static pressure transducer is placed slightly upstream of the blade passage to be studied. As the shock passes, it creates a sharp pressure spike. This sharp pressure spike, with the addition of a time delay circuit, triggers the shuttering of the CCD camera.

To produce the required known density field over a simple region of the flow, a variety of measurements need to be performed. Due to the unusual physical shape of the flowfield to be studied, it was determined that both upstream and wake measurements needed to be made. Because of limited optical accessibility, some of the upstream measurements need to be made very close to the blade surface and in the initial portion of the blade passage. Therefore, it was necessary to take into account any possible disturbance to the flowfield created by the probe. Because of this, total pressure measurements could not be made to quantify the strength of the passing shock. Therefore, a different approach had to be considered. Also, the survivability of the measuring instrument had to be considered, since after the shock has passed, it has been noted in the bench-top experiments that large Mylar fragments are thrown with high velocities out of the shock tube. It is highly likely that these fragments could damage any measuring instruments upstream of the blades. Also, again due to the high velocity of the moving shock, the expected frequency response of the measuring instrument must be included. Because of these factors, it was decided to use a hot wire specially constructed for use in this wind tunnel to calculate the temperature change of the flow as the shock wave passes upstream of the blades. These signals can also be synchronized off the spike on the upstream pressure transducer.

\subsection{Additional Measurement Instruments}

Aside from the required optical components which have been previously reviewed, there are quite a few system components dedicated to the control and direct evaluation of the tunnel flowfield. Some of these instruments are used not only in evaluating the required reference density field but also in the control of the tunnel operation. 
The tunnel flow rate is controlled with the output from a simple Pitot probe and a total temperature probe. This information is fed into a tunnel control system. The control system uses the total pressure to set the exit conditions from the cascade. This is possible because of the choked condition that exists within the cascade. In this case, the total upstream pressure is set to produce an isentropic exit Mach number of 1.23 .

An array of 41 static pressure taps were required for the evaluation of the reference density field. Fifteen of these taps were arrayed upstream of the blade row and the other 26 taps were arrayed in the blade wake region. As the shock passes upstream of the blades, both the expected total pressure and total temperature changes are computed. However, to increase the accuracy of this calculation, hot wire data is used as a correction to this calculation since a hot wire has a better time response and spatial resolution than the pressure transducers used. Therefore, a hot wire with small physical dimensions and high-frequency response was required. In order to keep track of the total pressure deficit in the wake region, a Pitot probe, equipped with a high frequency pressure transducer was used to compute the total pressure at each static pressure tap location in the wake region. One high-frequency pressure transducer was used to create a time mark to synchronize all of the data between separate runs. This transducer also acts as the triggering mechanism for the CCD camera. The static pressure transducers, total pressure transducer and trigger pressure transducer all require power supplies, bridge balances and amplifiers (to increase the sensitivity). One upstream total temperature probe is required to evaluate the total temperature of the studied turbine blade. It was decided to use the temperature probe already in place. The tunnel flow rate is controlled with the output from a simple homemade upstream total pressure probe. This system is adequate for tunnel control in this application. The control system has been tailored to produce an isentropic exit mach number of 1.23 from the linear turbine cascade. A waveform recorder is needed to capture the pressure and thermal data from the transient shock events. 


\subsection{Component Selection}

\subsubsection{Optical Components}

Only a few new optical components were required. Oriel, Edmund Scientific and Newport were all contacted. See Table 4.1 for the pricing information on the following items - new lens and pinholes for the spatial filtering of argon ion and HeNe laser beams. All of the companies had the required items in stock, with a one week delivery time. If the items are not in stock when ordered, all of the companies state a restock time between 3 to 4 weeks. The Oriel components were selected. This company has a reputation of producing high quality optical components at mid-range prices.

\subsubsection{High Frequency Pressure Transducers}

Due to the high frequency content of the pressure signal from a passing shock wave, a high natural frequency was the primary driving requirement in pressure transducer selection. In order to obtain accurate reference densities, not only did the time of the shock event have to be properly recorded, but the pressure rise through the shock needed to be faithfully followed. The signal 'ring' also needed to be minimized. Another important factor in pressure transducer selection was size. The static pressure tap array ( 15 taps) only covers a $0.635 \mathrm{~cm}(0.25 \mathrm{in})$ by $5.08 \mathrm{~cm}(2.00 \mathrm{in})$ area. Therefore, each sensing head needed to be no larger than $0.159 \mathrm{~cm}\left(\frac{1}{16}\right.$ of an inch).

In order to determine the specific natural frequency required, the sensing head diameter, shock speed $\left(340 \frac{\mathrm{m}}{\mathrm{s}}\right)$, and pressure rise time $\left(10^{-6} \mathrm{~s}\right)$ were used to compute the approximate frequency content of the shock. The computations showed the shock would have a frequency content up to $130 \mathrm{kHz}$. Figure 4.4 shows the transducer response spectrum from a shock tube input computed by Endevco. This shows the main frequency content of a passing shock wave to be centered at $120 \mathrm{kHz}$. Therefore, it is desirable to choose pressure transducers that will faithfully represent a signal up to $120 \mathrm{kHz}$.

Elastic force pressure transducers and piezoelectric transducers are generally represented as spring-mass-damper systems. The transfer function for such a system is 


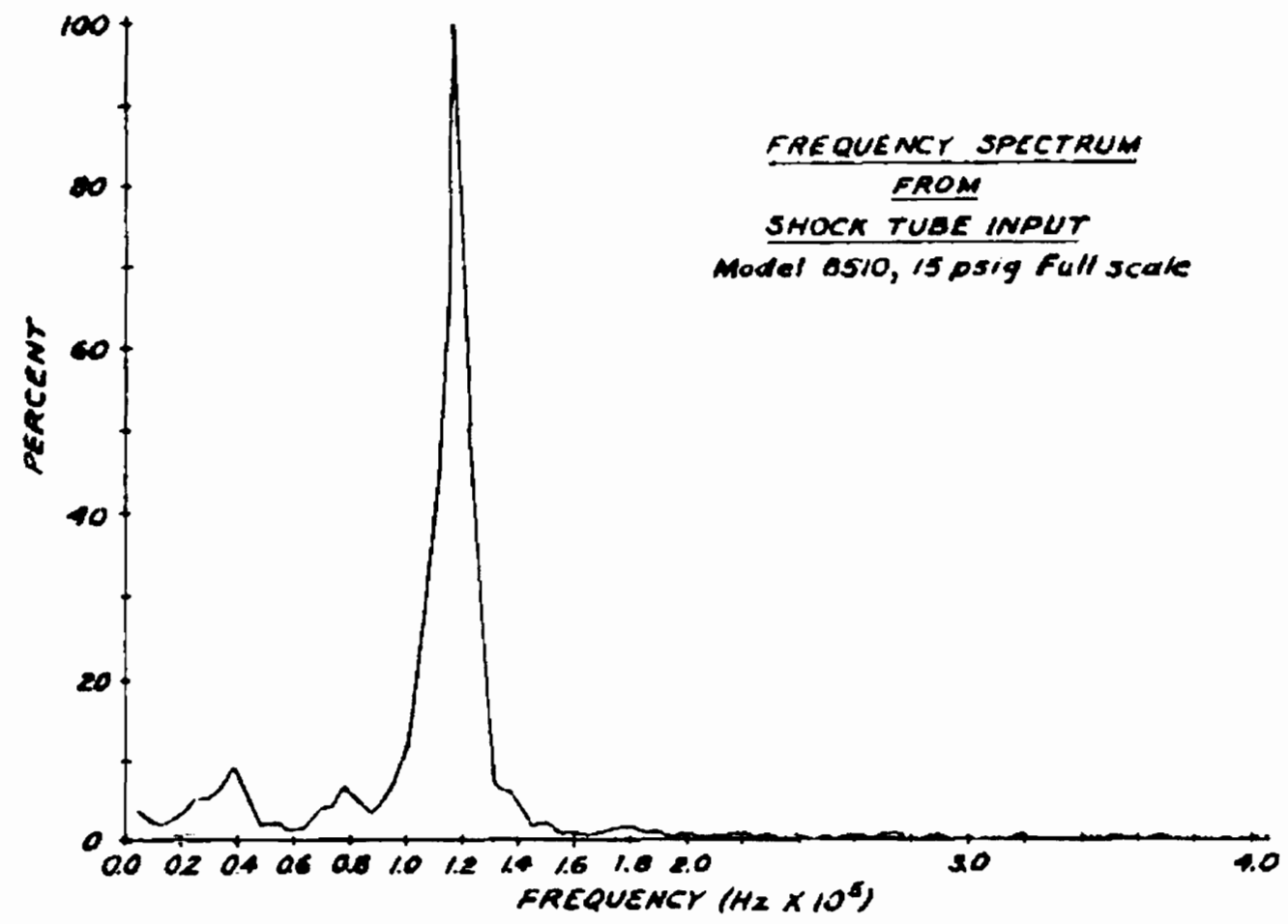

Figure 4.4: Shock Wave Frequency Spectrum 
given below:

$$
\left\|\frac{e_{0}}{K e_{i}}\right\|=\frac{1}{\frac{s^{2}}{\omega_{n}^{2}}+\frac{2 \zeta s}{\omega_{n}}+1}
$$

where $\left\|\frac{e_{0}}{K e_{i}}\right\|$ is the signal amplification of the system, $w_{n}$ is the transducer natural frequency and $\zeta$ is the damping in the transducer. Figure 4.5 shows the typical frequency response of a high-frequency pressure transducer. It is apparent that a pressure transducer with a natural frequency of at least $600 \mathrm{KHz}$ is required to keep the unwanted signal amplification low $(<0.5 \mathrm{~dB})$.

Three companies were contacted about high-frequency pressure transducers. Table 4.2 summarizes the critical properties of the recommended pressure transducers. The Kulite XCQ-062-100 Ultraminature IS pressure transducer (Fig. 4.6) was chosen as the most suitable. Though the Kulite transducer is the least sensitive of the three, it is the only transducer found with a natural frequency approaching that required. This transducer is a solid state pressure device incorporating a diffused four-arm Wheatstone bridge on the surface of a silicon diaphragm. The XCQ-062-100 transducer has a pressure range from 0-100 psig. The highest expected gage pressure in this unsteady flowfield is computed to be 40 psig. Figure 4.7 shows the expected frequency response of the system (assuming a damping of 0.1 ).

The dynamic response of this system to an impulse input is shown in Fig. 4.8. The impulse response was computed to determine the duration of the pressure 'ring'. Since the system is extremely under-damped ( $\zeta=0.1$ was used as an estimate), the transducer signal will oscillate about the mean actual value for many periods after the initial excitation. To reduce the effect of these oscillations, shadowgraph studies were used to time the interferograms such that no shocks are directly over the reference density field when measurements are taken.

\subsubsection{Passage Hot-Wire}

A small, custom hot-wire probe from Auspex corporation was used for making measurements near and within the blade passages. This probe has been made as small as possible to minimize blockage in the tunnel, with a $0.35 \mathrm{~mm}$ frontal height on the 
Table 4.1: Optical Component Sources

\begin{tabular}{|l|l|l|l|}
\hline Component & Company & Part Number & Price $(\$$ each) \\
\hline \hline 4 in square mirror & Edmund Scientific & G40,042 & 20.05 \\
\hline & Oriel & 45721 & 107.00 \\
\hline & Newport & $60 \mathrm{D} 10 \mathrm{AL} .2$ & 623.00 \\
\hline Mirror Holder & Edmund Scientific & G36,482 & 116.00 \\
\hline & Oriel & 12660 & 79.00 \\
\hline & Newport & $625-\mathrm{RC} 6$ & 347.00 \\
\hline Spatial Filter & Edmund Scientific & G39,976 & 395.00 \\
\hline & Oriel & 15221 & 898.00 \\
\hline & Newport & M-900 & 716.00 \\
\hline X10 Objective & Edmund Scientific & G45,045 & 80.00 \\
\hline & Oriel & 15500 & 114.00 \\
\hline & Newport & included above & \\
\hline $25 \mu \mathrm{m}$ Pinhole & Edmund Scientific & G39,886 & 58.00 \\
\hline & Oriel & 13580 & 89.00 \\
\hline & Newport & included above & \\
\hline & & & \\
\hline
\end{tabular}

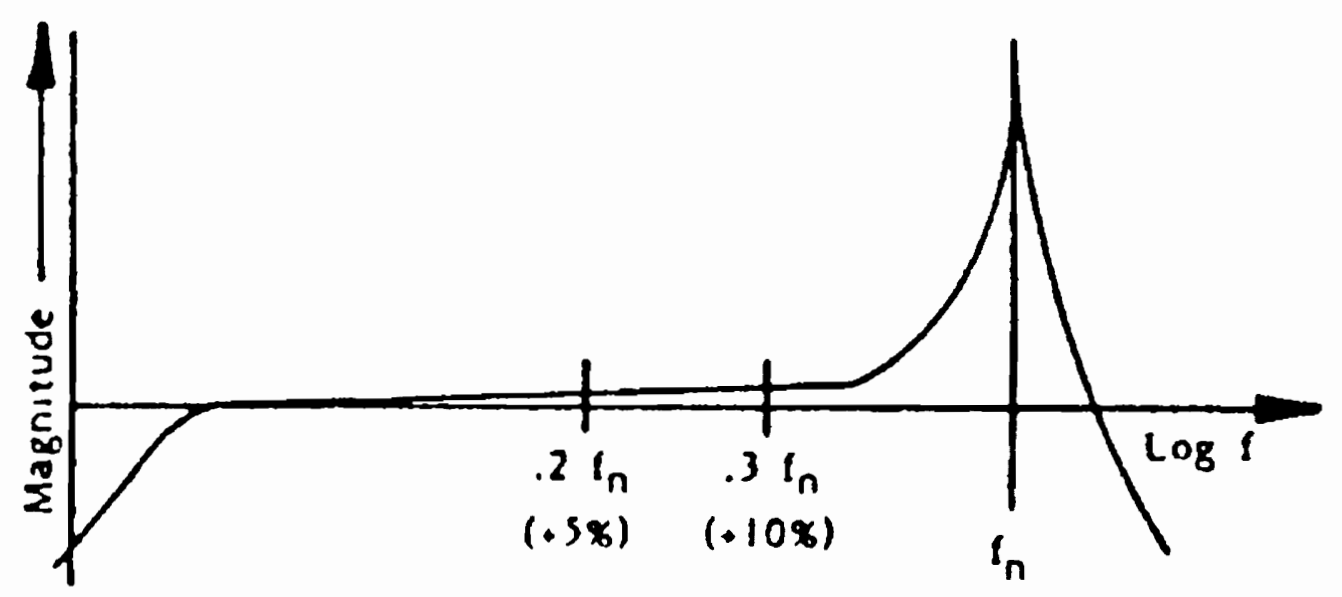

Figure 4.5: Representative Pressure Transducer Gain 
Table 4.2: Pressure Transducer Sources and Critical Properties

\begin{tabular}{|l||c|c|c|}
\hline Company & Kulite & Endevco & Dytran \\
\hline \hline Part Number & XCQ-062-50 & $8415-50$ & $2100 \mathrm{~V} 1$ \\
\hline Natural Frequency & $1000 \mathrm{kHz}$ & $320 \mathrm{kHz}$ & $300 \mathrm{kHz}$ \\
\hline Sensitivity $(\mathrm{mV} / \mathrm{psi})$ & 2.0 & 6.3 & 8.0 \\
\hline Resolution & infinite & - & - \\
\hline Linearity, Hysteresis & $0.5 \mathrm{mV}(\max )$ & $1.575 \mathrm{mV}$ & $8 \mathrm{mV}(\max )$ \\
\hline Thermal Sens. Shift & $0.05 \mathrm{mV} /{ }^{\circ} \mathrm{F}$ & $0.0315 \mathrm{mV} /{ }^{\circ} \mathrm{F}$ & $0.24 \mathrm{mV} /{ }^{\circ} \mathrm{F}$ \\
\hline Repeatability & $0.1 \mathrm{mV}(\max )$ & $0.315 \mathrm{mV}$ & - \\
\hline
\end{tabular}

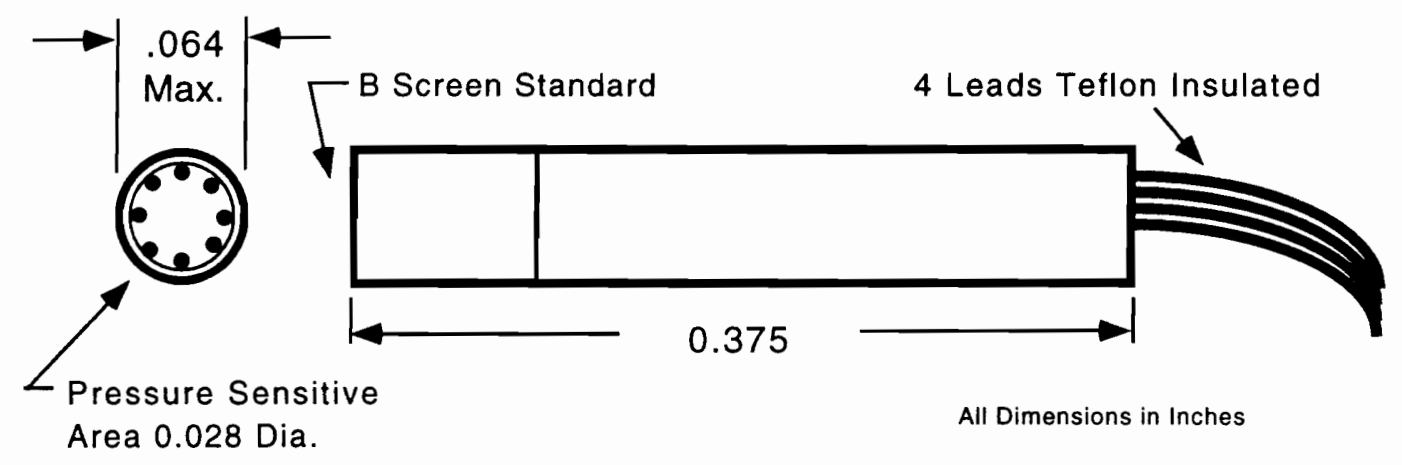

Figure 4.6: Kulite XCQ-062-50 Pressure Transducer 

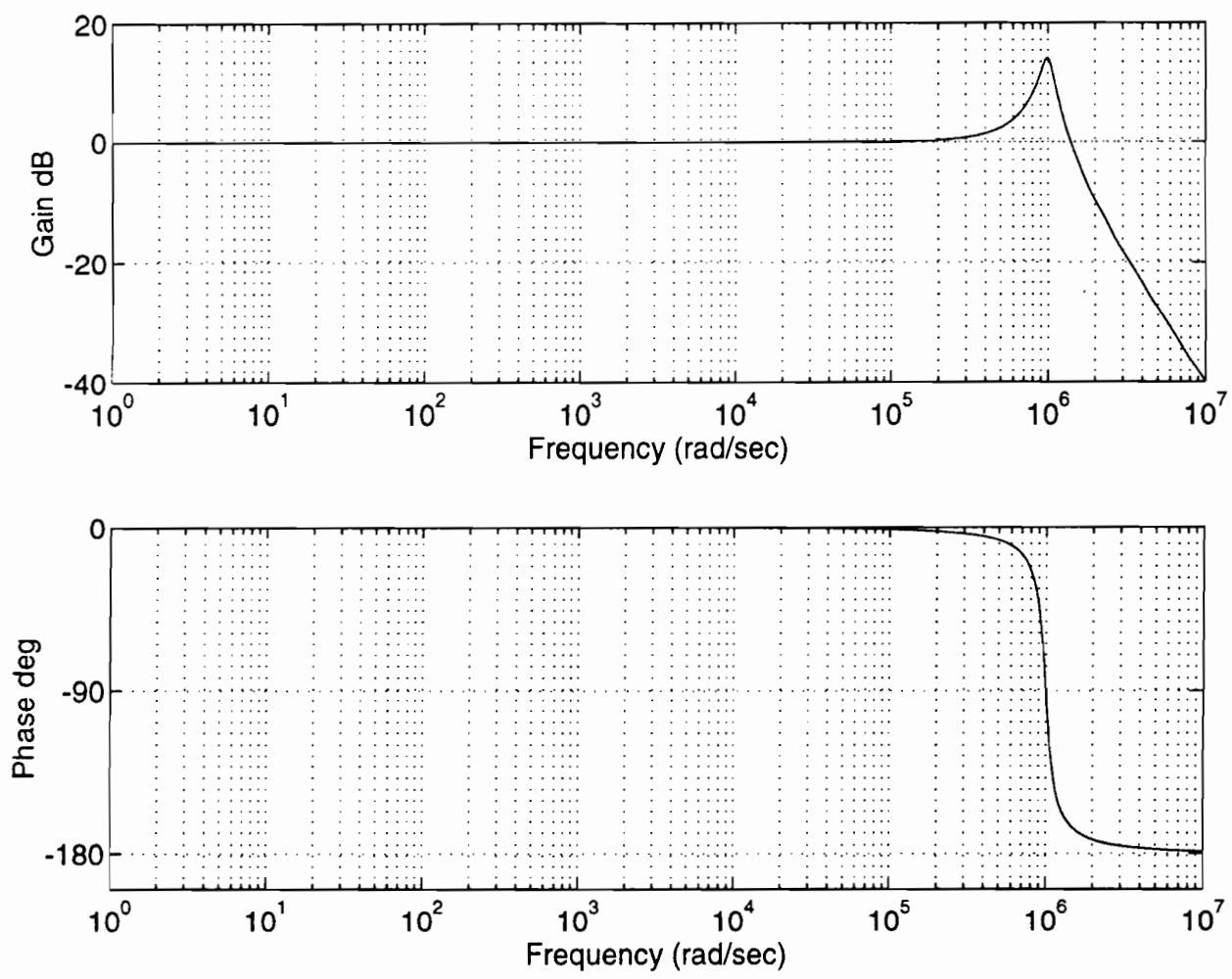

Figure 4.7: Pressure Transducer Frequency Response

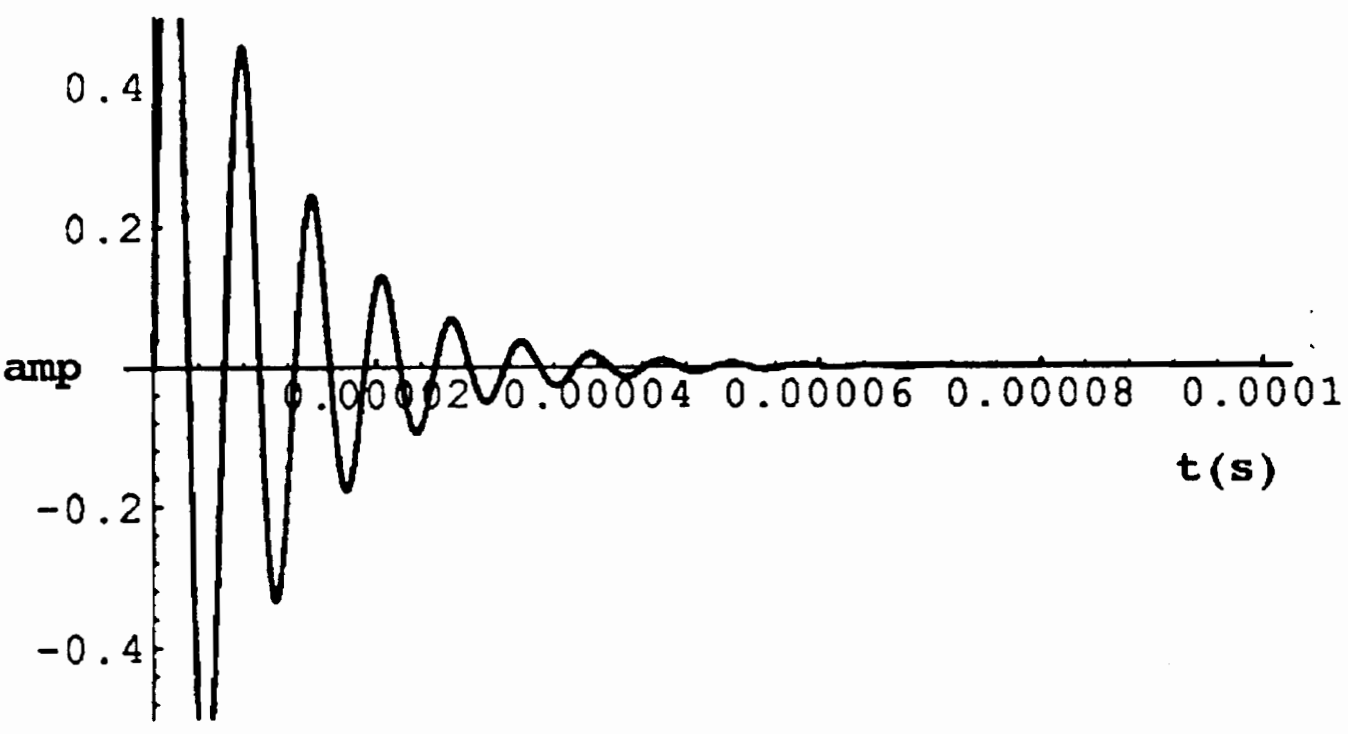

Figure 4.8: Dynamic Response of Pressure Transducer to an Impulse Input 
support extending from the endwall to the wire prongs. This is a custom probe with $3.175 \mathrm{~mm}\left(\frac{1}{8}\right.$ in $)$ stainless steel prongs on the small body described above. The $5 \mu \mathrm{m}$ annealed Tungsten wire parallel to the probe body is welded by hand in the AOE hot-wire lab.

Before any tests are performed, the frequency response and the wire stability must be investigated. These response tests were performed by David Holmberg of the Mechanical Engineering Department of Virginia Tech. The hot wire was driven by the TSI, Inc. IFA-100. During all of these tests, except for the frequency response estimation, the test signal from the IFA-100 was set to $20 \mathrm{KHz}$ and the wire was set to an over-heat ratio (OHR) of 1.8. The bridge voltage from the anemometer was sent to an oscilloscope to view the triggered time series waveform. This signal was also sent to a HP 3562A Dynamic Signal Analyzer, where the frequency spectrum could be viewed from 0 to $100 \mathrm{kHz}$. A square-wave test signal (with a range of 1 to $43 \mathrm{kHz}$ ) from the IFA-100 was applied to the wire to allow viewing the frequency response and stability of the wire. The wire was tested in no-flow conditions, as well as in both the laminar and turbulent regions of the jet of a TSI HW calibrator and in the tunnel upstream of the blades and in the passage.

The IFA-100 anemometer allows setting the OHR via an Operating Resistance knob on the front panel. Additionally, there are two other knobs used to tune the frequency response of the wire, namely the Bridge Compensation (BC) knob and the Cable knob. TSI provides recommended BC settings for their standard probes and gives procedures for setting the Cable bridge compensation for custom probe designs. In general, modifications to the recommended settings should be made while the wire is in the actual test conditions. The cable setting is largely responsible for the stability of the wire. This stability refers to the narrow range, analogous to a plateau, around which the wire is unstable. If the wire goes unstable, frequency oscillations occur, evidenced by a lower output mean voltage, wild fluctuations of the oscilloscope trace and broad-band noise in the frequency spectrum. The following tests were performed to examine the proper control settings for the hot wire.

First, the hot wire was tested initially in no-flow conditions. It was found that when the $\mathrm{BC}$ was set to 90 and the Cable adjusted, a textbook case pulse was found 
with a width of $20 \mu \mathrm{s}$. When the frequency of the test signal was increased, the waves of the test signal began to merge on the oscilloscope. Also, the amplitude of the wire response to the test signal started to decay at a frequency of $35 \mathrm{kHz}$. This is a good estimate of the wire frequency response at no-flow conditions. It was noted that increasing the Cable setting caused more noise in the frequency response.

Next, the hot wire was tested in the TSI calibrator. In the jet flow, it was necessary to turn both the Cable setting and the $\mathrm{BC}$ down to get a desired test signal response with flow. The $\mathrm{BC}$ seemed to produce the best signal when set at 60 . At this setting, there were no noise peaks in the frequency spectrum. As BC was increased, however, the amount of higher frequency noise grew until the levels on the high end of the frequency spectrum were as high as those on the low end.

Finally, the hot wire was tested in three different tunnel locations. First, the hot wire was tested in the tunnel inlet flow. With BC set at 60 and the Cable adjusted below the point leading to frequency oscillations (OSC), a frequency spectrum was obtained similar to that found with the TSI calibrator jet. It was also found that at the optimal settings for the BC and Cable, the test signal had a peak and a double dip, as opposed to the single dip found in the textbook case. The differences between the flow and no-flow settings on the IFA-100 were found to be large. The test signal with flow had the before-mentioned double dip and a peak width of $4 \mu \mathrm{s}$, while at no-flow conditions the same signal had a peak width of more than $20 \mu$ s. The peak width of $4 \mu \mathrm{s}$ for the flow case implies a frequency response better than $150 \mathrm{kHz}$.

Next the hot wire was tested in the passage and near to the suction surface of the blade. Here, it was found that running with $\mathrm{BC}$ equal to 60 produced some high frequency noise, however if $\mathrm{BC}$ is reduced to 50 the noise disappears. At no-flow at these settings, the Cable is slightly more than $\frac{1}{2}$ a turn back from OSC. Finally, the hot-wire was tested in the blade passage and near the pressure surface of the blades. Here, it was found that the optimum conditions are produced by a BC setting of 60 and the Cable still $\frac{1}{2}$ turn back from OSC. Therefore, it was determined that the optimal settings for this hot-wire are with a $\mathrm{BC}$ of 60 (possibly 50 near the blade suction surface) and the Cable knob slightly more than $\frac{1}{2}$ turn back from complete OSC. This should produce a frequency response greater than the needed $140 \mathrm{kHz}$. 


\subsubsection{CCD Camera}

Charge coupled devices (CCD) were introduced in 1970 by Boyle and Smith of Bell Laboratories in an attempt to find a silicon based electronic analog for magnetic bubble memory. It was rapidly realized, however, that the CCD's were capable of performing a large number of electronic functions. CCD use quickly expanded into many fields including imaging, signal processing, logic functions and analog and digital memory. CCD's have by far made their greatest impact and contribution in the area of visible imaging. In addition to the advantages of solid-state construction, low power consumption and automatic analog-to-digital (A/D) conversion, CCD cameras offer a number of advantages over conventional photographic film. Among these advantages are extremely high quantum efficiency, broad spectral bandwidth, large dynamic range and high photometric accuracy [36].

Three companies were contacted for pricing and availability on high-speed CCD image intensified cameras. Two companies (Cordin and JC Labs) stated that a CCD camera suitable for this application would have to be custom made. For both custom designs, a construction time of 60-90 days would be required. Stanford Computer carries a CCD camera suitable for this application in stock. This company quoted a delivery time of 2 to 4 weeks depending on system availability. Table 4.3 shows the quoted pricing, all above $\$ 15,000$. Because of the high costs and long delivery times required, an alternate source was sought.

Dr. U. Vandsburger, of the Virginia Tech Mechanical Engineering Department, was found to be in possession of a Stanford Computer 4 Quick 05/5-25 camera. This camera is slightly (20\%) less sensitive than the camera recommended by Stanford computer for our application. This is due to its $5 \mathrm{~ns}$ minimum shutter time capability - as opposed to the $100 \mathrm{~ns}$ minimum shutter time for the 100-25 model. However, the Quick 05/5-25 should be sensitive enough, because of its high gain capabilities, for this application.

Important parameters of the Stanford CCD camera are:

1) wide range of exposure times from $5 \mathrm{~ns}$ through $\mathrm{DC}$

2) wide spectral range $(130 \mathrm{~nm}-920 \mathrm{~nm})$

3) $1,000,000,000: 1$ dynamic range 
4) Peltier cooling

5) frame grabber and software

6) $\mathrm{MCP}$ Intensifier - $10^{7}$ photonic gain possible $5 \mu \mathrm{Lx} @-6 \mathrm{~dB}$ and $6 \mathrm{~mm} \times 4.5 \mathrm{~mm}$ image area

7) $604 \times 576$ pixels (super VHS)

8) signal to noise $46 \mathrm{~dB} \min @ 0.5 \mathrm{mLx}$

9) frame grabber jitter, $25 \mathrm{~ns}$

10) trigger delay, $40 \mathrm{~ns}$

\section{Image Intensifier}

A circular cathode is set directly in front of the proximity-focused micro-channel plate $(\mathrm{MCP})$, as in Fig. 4.9. Here, the light image entering the camera system is transformed into an electron image and focused onto the intensifier. Microchannel plate image intensifiers are made up of three main components: a photocathode, an anode and the micro-channel plate. The MCP device is based around photomultiplier tube technology where a single photon event impacting on the tube emits a single electron into the tube internal wall. These walls are in turn coated with an electro-emissive surface. The single electron quickly produces a cascade of electrons, thus generating a large electron signature from a single photon event. The intensifier micro-channel plate is made up of numerous independently working photomultipliers in the form of very narrow hollow glass cylinders, arranged to form a plate. This intensified image of secondary electrons is delivered with extremely high spatial resolution onto a phosphor screen. The phosphor screen, in turn, emits a stream of photons proportional to the electron signature. Thus, an intensified stream of photons is provided as input to the CCD array [37].

\section{Photocathode and Shutter}

The MCP shutter is activated by the application of a negative voltage of $200 \mathrm{~V}$ to the photocathode. The duration of this voltage pulse determines the opening time of the CCD shutter. 


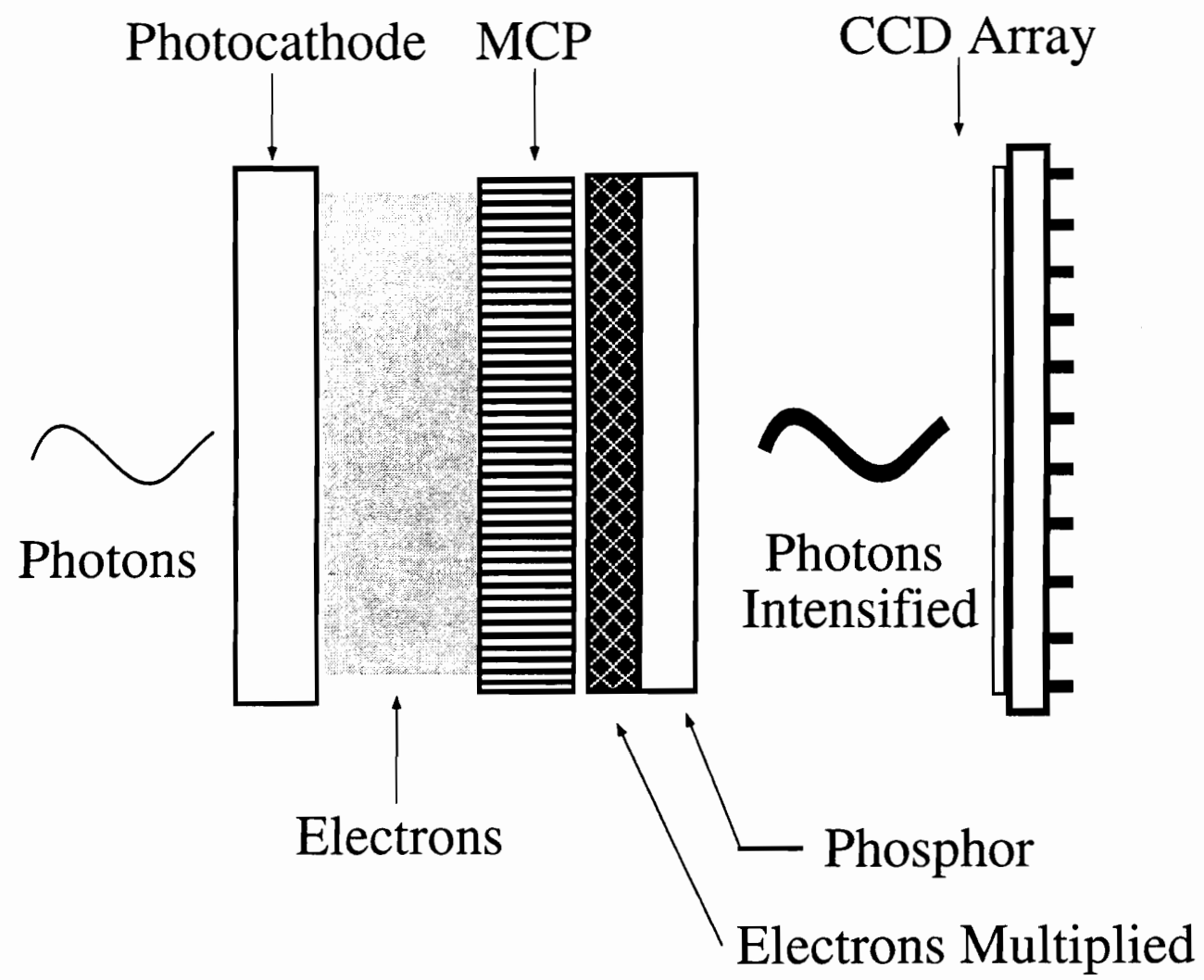

Figure 4.9: Micro Channel Plate Design [Sturz, 1995] 


\subsubsection{Laser}

The suitability of several laser sources for this experiment was investigated. These sources included a Helium-Neon, an Argon Ion gas laser and a Copper Vapor laser. Each of these types were evaluated based on spatial and temporal coherency, available power, pulse length, ease of setup, survivability and availability (with the latter three factors being of primary importance).

The possibility of using the Aerospace Department Copper Vapor Laser (CVL) was first investigated. As mentioned before, the temporal coherence length of this type of laser is poor due to the Doppler shift associated with the high temperatures the laser operates at - approximately $1500^{\circ} \mathrm{C}$ [38]. These high temperatures are necessary in metal vapor lasers, since it is necessary to heat the metallic atoms to a temperature enabling vaporization within the discharge tube. The construction of metal vapor lasers differs from that of other atomic lasers in that it is necessary to make provisions for containing the metal, heating it to a vapor and then distributing this vapor within the laser discharge tube. Typically, the solid metal is contained in one end of the tube near the anode and is heated to vaporize the necessary amount of metal into the discharge tube. As the metal vapor enters the discharge tube, the positively charged ions are attracted toward the cathode and drift along the length of the tube bore to form the active laser medium. Since there is no discharge in the cathode region, the temperature is lower. Thus, the metal vapor condenses on the tube. This process of continually transferring the metal from the anode to the cathode is known as cataphoresis pumping. Research performed by Oxford Lasers found that to improve the coherence length of this laser system beyond $5 \mathrm{~cm}$ would require pumping of a prototype injection seeded Ti-Sapphire laser [38]. Despite the high output $(45 \mathrm{~W})$ and pulse capabilities, the intricate setup required to create the necessary coherence length made this laser unsuitable for this application.

Next, a higher powered argon ion laser from the Mechanical Engineering Department of Virginia Tech was tested. An argon ion laser, as opposed to the CVL, is a noble ion gas laser. Noble ion gas lasers are the most powerful visible lasers that operate continuously. Outputs of several watts in a single line can be obtained from some of these lasers. Despite the fact that the rare gases have filled electron shells 
and, consequently, do not normally combine chemically with other elements, they have exhibited the remarkable ability to support stimulated photon emission. By passing a very high current (on the order of 15 to $50 \mathrm{amps}$ ) through the discharge tube, the gas is ionized and an inverted population of the energy levels is achieved. As the electrons drop back down to available lower energy state, photons of a specific wavelength (corresponding to the energy difference between the two transitioned energy levels are emitted). The two strongest lines of the argon ion laser occur at 488.0 $\mathrm{nm}$ and $514.5 \mathrm{~nm}$, in the blue and green portions of the spectrum, respectively.

The tube design of an argon ion laser is complex (much more so than the HeliumNeon laser to be discussed later). Generally speaking, many lines are produced simultaneously with the usual mirror arrangement, although they all have widely different powers and thresholds. Therefore, a prism cut at Brewster angles is generally incorporated as part of the cavity. This window makes the losses very high for all the wavelengths except one and the laser can be easily tuned to the desired wavelength. The available output power is generally limited by the rate at which heat can be dissipated from the system, so the anode and discharge tube are cooled by the circulation of water. This unfortunately creates a very bulky and impractical system in the tunnel environment.

With the addition of the intensified CCD camera to the interferometric system, the use of the HeNe laser was investigated. The HeNe is a type of atomic laser, utilizing the transition between energy levels of non-ionized atoms. The basic construction of a CW (continuous wave) HeNe laser is fairly simple. The essential elements of this basic laser are the discharge tube containing the 10 parts Helium to 1 part Neon gas, the power supply and the reflecting mirrors. HeNe lasers typically have power outputs of less than $50 \mathrm{~mW}$, but they do exhibit coherence lengths on the order of meters. Though the intensity of the light from a HeNe laser is only a small fraction of that output from typical Argon Ion or Copper Vapor lasers, the light intensification provided by the MCP intensifier proved to make the higher powered lasers unnecessary. A bench top test of the system with Spectra-Physics Stabilite Model 120 Helium-Neon laser used as the light source produced viable CCD images with exposure times of less than $100 \mathrm{nsec}$ when the MCP gain was set to approximately 
$750 \mathrm{~V}$ [39]. Since viable interferograms could be produced by the more robust HeNe laser, it was chosen as the lights source for this work.

\subsubsection{Timing Delay Circuit}

A simple time delay circuit to trigger the frame grabber of the CCD camera was designed. Figure 4.10 shows the electrical diagram for the circuit. The delay time in this circuit is determined by the circuit resistance, and it can be adjusted with a 10 turn potentiometer. The signal from a Kulite transducer, placed at tap T1, was used as input to the time delay circuit. The timing circuit could be trigged manually by activating the auto-range switch on the signal conditioning system. This trigger signal and the TTL output from the timing circuit were both recorded on the LeCroy recorder. The necessary time delay of the circuit could be set before each run in this manner, reducing the effects of any temperature drift in the TTL circuit.

\subsubsection{Strain Gage Balance and Amplifier}

Due to the low voltages produced from the Kulite transducers (on the order of 50 $\mathrm{mV}$ ) amplification of the signal is required. A Measurements Group Model 2310 Signal Conditioning System is used to condition and amplify signals from various transducers. This system is sufficient for use with the Kulite pressure transducers. Another attractive aspect is the large number of these amplifiers available in the laboratory.

The signal conditioner not only contains an operational amplifier but also a bridge balance, shunt calibration and bridge excitation capabilities. A simple bridge circuit illustrating sensitivity, balance and calibration features is shown in Fig. 4.11.

Shunt calibrators are used to calibrate the bridge directly by introducing a known resistance change and noting the effect on the bridge output. The bridge balance provides a way to adjust the output voltage to precisely zero when the measured physical quantity is zero.

The following are important parameters of the signal conditioning system: 
Table 4.3: CCD Camera Sources

\begin{tabular}{|l||c|c|c|}
\hline Company & Phone & Part Number & Quoted Price \\
\hline \hline Stanford Computer & $(415) 494-6711$ & 4 Quick 05/100-25 & $\$ 26,400$ \\
\hline Cordin & $(801) 972-5272$ & Custom Item & $\$ 15,000$ \\
\hline JC Labs & $(415) 967-3431$ & Custom Item & $\$ 20,000$ \\
\hline
\end{tabular}

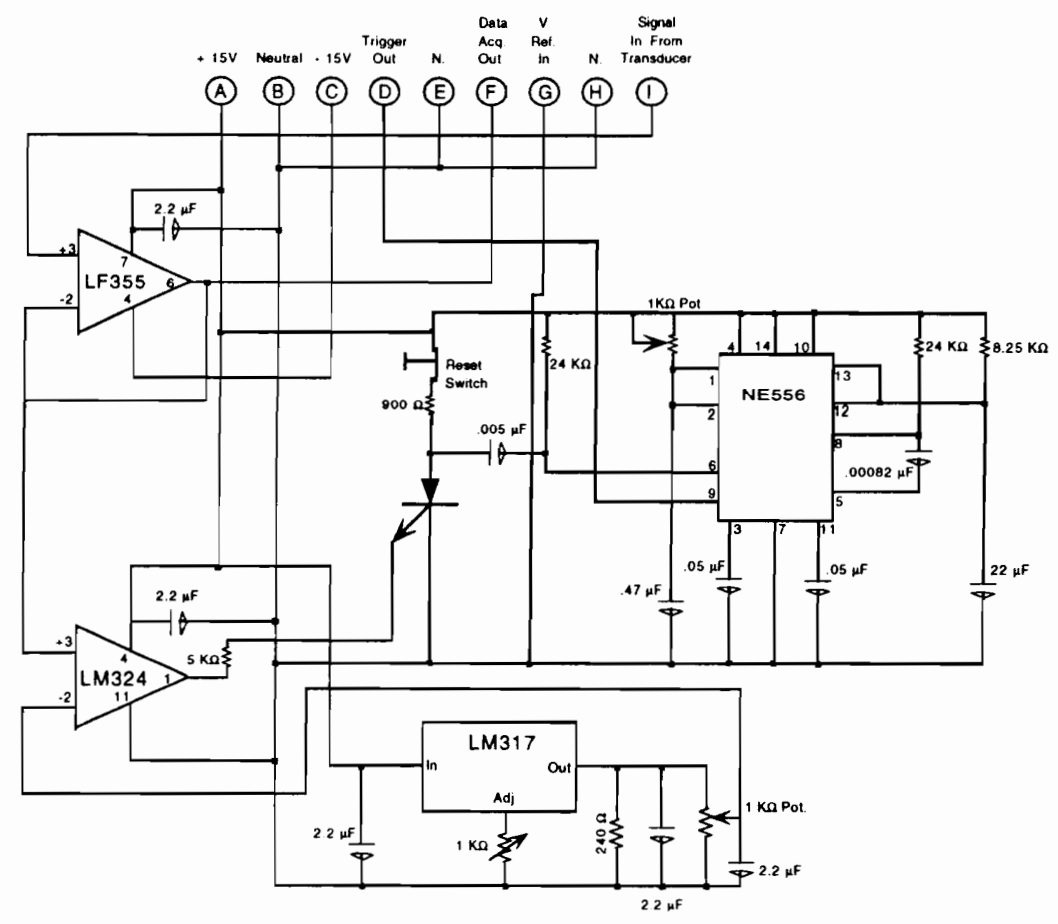

Figure 4.10: Time Delay Circuit 
Excitation - 12 settings:0.5, $0.7,1,1.4,2,2.7,3.5,5,7,10,12$, and $15 \mathrm{Vdc}+/-1 \%$ $\max$.

Bridge Balance - (auto ranging) $+/-5000 \mu \epsilon(1 \%$ bridge unbalance or $2.5 \mathrm{mV} / \mathrm{V})$, resolution $12.5 \mu \epsilon(0.006 \mathrm{mV} / \mathrm{V}) 2$ second, typical balance time Essentially independent of excitation and amplifier gain

Amplifier - Gain: 1 to 11000 continuously variable Frequency Response: dc $-65 \mathrm{KHz}$, $-3 \mathrm{~dB}$ typical Common Mode Rejection: 90dB, typical Stability: $+/-2 \mu \mathrm{V} / \mathrm{C}$ RTI Noise: 0.5 - $50 \mathrm{KHz}: 5 \mu \mathrm{V}$ rms, $\max$

\subsubsection{Waveform Recorder}

A LeCroy Model 6810 Waveform Digitizing/Recording system has been used in prior work in our laboratory to record shock passing data. The system allows sampling rates of 1 million samples per second on 4 channels, simultaneously. This sample rate provides a factor of 8.3 over the largest expected signal frequency. This factor is above the 6.3 factor defined by the Nyquist frequency, but is slightly below the desired factor of 10 . If the sample rate is inadequate, a sampling rate of 2 million samples per second can be achieved by only utilizing two of the four slots available per module.

Some important information on the LeCroy Model 6810 Waveform Recorder follows:

- 12 bit analog-to-digital converter providing vertical resolution of 1 part in 4096 or $0.025 \%$ and $2 \%$ absolute accuracy.

- 8 Million sample memory per module, providing 2 full seconds of recording time per channel at 1 Million sample per second sample rate.

- provides slope, window, and hysteresis triggering.

- range, independently programmable for each channel, $100 \mu \mathrm{V}, 250 \mu \mathrm{V}, 500 \mu \mathrm{V}, 1$ $\mathrm{mV}, 2.5 \mathrm{mV}, 6.25 \mathrm{mV}, 12.5 \mathrm{mV}, 25 \mathrm{mV}$ per LSB.

- temperature stability, better than $50 \mathrm{ppm}$ per C.

- overload recovery, recovers to within $\pm 0.1 \%$ full-scale in $1 \mu$ sec.

- DC gain linearity, $<0.1 \%$. 
- Dynamic accuracy: SNR $63 \mathrm{~dB}$, Harmonic distortion -63 dB.

- Trigger signal must have a minimum duration of $50 \mathrm{nsec}$.

\subsection{Problem Issues}

Some of the problem issues in this system include:

1) The high cost of the optical components requires the use of less expensive, less suitable components. This includes using a continuous laser instead of a pulse laser and using an in-house CCD camera instead of purchasing one specifically designed for this application.

2) By far, the largest contribution to the uncertainty comes from the reference pressure data. This is mainly due to the low sensitivity provided by the Kulite transducers. If a pressure transducer could be found with the same natural frequency, but more sensitivity, the overall uncertainty of the system would be greatly improved.

3) The size and fragility of the laser makes it difficult to use in the wind tunnel environment.

4) Calibration is difficult to obtain for the Kulite transducers; therefore, the manufacture's calibrations must be used.

5) The large temperature fluctuations in the tunnel environment can cause calibration drift in the Kulite pressure transducers as well as some drift in the power and wavelength provided by the laser.

7) Many of the optical components are very fragile. The leads of the Kulite transducers are easily broken and continuity lost.

8) If the manual gain on the CCD camera is adjusted at too low values, this could simulate 'too low input brightness'. The result could be a 'burn in' of the photocathode.

9) The circular surface of the MCP in the CCD camera can be seen and handled after removal of the optical lens assembly. Care must be taken while manipulating the lenses. Damage to the photocathode of the MCP could take up to 90 days to repair. 


\subsection{Human Factors}

By far, the most delicate and dangerous instrument found within the experiment is the Helium Neon laser and the light produced by it.

Hazards associated with lasers include: 1) exposure to laser radiation may result in damage to skin or eyes, 2) gaseous substances contained by the laser are chemical hazards and 3) high-powered lasers operate at extremely high voltages. Secondary hazards include: x-ray radiation from faulty power supplies, high noise levels, Pressurized lamps, cylinder, hoses, etc..., EMI/RFI emissions from faulty equipment.

Both the tunnel and the shock tube produce high noise levels requiring the use of hearing protection equipment. Lastly, the shock tube is a highly pressurized system, requiring safety precautions.

\subsection{Future Improvements}

Some systems were not investigated due to time constraints. Oriel was contacted about coating the wedge plate to decrease the almost $80 \%$ light loss through this component, however this will require more study. The presence of plane polarized light from the laser changes the reflectance properties of dielectric coatings. 


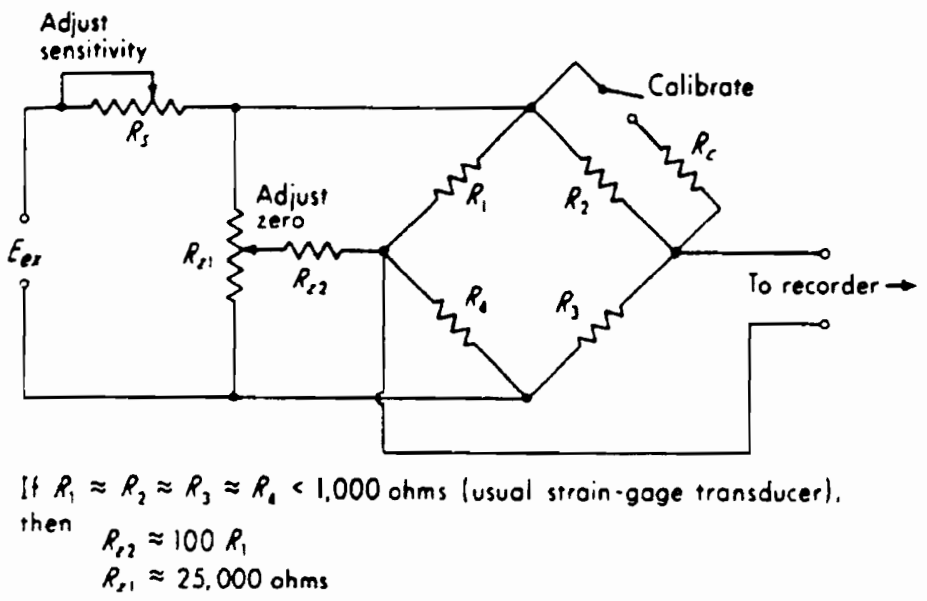

Figure 4.11: Bridge Circuit 


\section{Chapter 5}

\section{Data Collection and Reduction}

\subsection{Data Collection}

\subsubsection{Steady, Unheated and Heated Reference Density Field Collection}

The steady, upstream, unheated an heated reference density field collection only required the evaluation of the static pressure at the 15 specified upstream points. Special cascade Plexiglas endwalls were precision machined with $1.73 \mathrm{~mm}$ (0.068 in) diameter holes to allow fitting of the Kulite pressure transducers flush with the cascade interior face. These measurements, coupled with the upstream total pressure and total temperature measurements collected for all tunnel runs, can be simply reduced to a density value, assuming the flow is isentropic ahead of the blade row. This is a valid assumption, since the total pressure and temperature losses in a transonic cascade are negligible upstream of the trailing edge shocks.

Since it was desirable to standardize the data collection procedure, the steady static pressure measurements were collected using the Kulite pressure transducers, as opposed to standard static pressure taps. The Kulite pressure transducers used for all of the steady data collection - both heated and unheated - were B-Screened type. The addition of a screen to the transducer reduces the maximum frequency response from $140-150 \mathrm{kHz}$ for the unscreened type to near $50 \mathrm{kHz}$ for the screened 
type. However, because the screened pressure transducers are more robust, it was decided the screened pressure transducers would be used where the greater frequency response of the delicate unscreened transducers was not required.

For both cases the pressure transducers were connected to the Model 2310 Signal Conditioners. These signal conditioners allowed for bridge balancing, signal amplification and provided the transducer voltage excitation. All of the Model 2310 Unit balances were set to a nominal amplification factor of 100 . The amplification increased the range of the transducer signal from $0-50 \mathrm{mV}$ to $0-5 \mathrm{~V}$.

From the Model 2310 Unit, the signal was then low-pass filtered at $10 \mathrm{kHz}$ for the unheated case and $50 \mathrm{kHz}$ for the heated case and sampled by the LeCroy digital waveform recorder. Five runs were performed at each of the 15 static pressure tap locations.

\subsubsection{Upstream, Unsteady, Unheated Reference Density Field Collection}

The unsteady, upstream, unheated reference density field collection required the evaluation of both the static pressure and velocity at the 15 specified upstream points. For the static pressure measurements, the Kulite transducer holes machined for use in the steady measurements were used. In the opposite endwall, diameter holes were machined to allow mounting of the passage hot wire probe through the endwalls. The hot wire probe was positioned such that the probe body was situated downstream of the initial incident passing shock and most of the strong reflections. It also was extended into the span so that the wire was located mid-span. These measurements will be combined with the initial total temperature and pressure measurements collected for all tunnel runs to produce a time history of the pointwise density values at the 15 chosen locations.

The Kulite pressure transducers were connected to the Model 2310 Signal Conditioners, as in the steady cases and then run through the filter on the IFA-100. The hot wire was connected directly to the IFA-100, where the BC and Cable were set. Both transducer signals were loss-pass filtered at a frequency of $200 \mathrm{kHz}$. Because 
of the concerns about damage being caused to the Mylar fragments, only three runs were made at each of the locations for each of the two transducers. A trigger Kulite transducer situated slightly upstream of the studied passage was used to trigger the data collection on the LeCroy digital waveform recorder. The unsteady data was recorder at a rate of 2 million samples per second.

\subsubsection{Unsteady Interferograms}

One of the first decisions to be made was the times at which the interferograms would be taken. To more easily facilitate this process, a shadowgraph study of the progression of the shock through the blade passage was undertaken. Using the same timing delay circuit mentioned earlier, a series of shadowgraphs was taken. Figure 5.1 is a rendering of the progression of the shock through the section at various delay times. It should be noted that as the shock travels along the blades, this shock pattern will be repeated with decreasing strength at each blade row.

In the first picture, the shock can be seen to have already entered the blade passage. This was the least amount of delay time the setup would allow, therefore the trigger Kulite transducer has been moved further upstream for interferometric image collection. As the shock continues to move along the blade row, it encounters the suction surface of the lower blade in the studied blade passage, reflects off the surface and begins to move upstream. This is shown in the second drawing of Fig. 5.1. In the next drawing, the reflected shock has moved further into the blade passage toward the pressure surface of the upper blade in the studied passage. In Fig. 5.1, the reflected shock has now reached the pressure surface of blade number 1 and has in turn reflected off that surface, and again reversed direction. These reflections continue for several $\mu$ seconds.

The exposure time for all CCD images was $100 \mathrm{~ns}$ at a gain of $750 \mathrm{~V}$. The time delay circuit was easily calibrated using the LeCroy system while actually running the tunnel. This allowed the calibration to be conducted as close to the actual experimental conditions as possible. This was performed by artificially inducing a voltage spike by auto-balancing the amplifier. This voltage spike was recorded on the LeCroy 

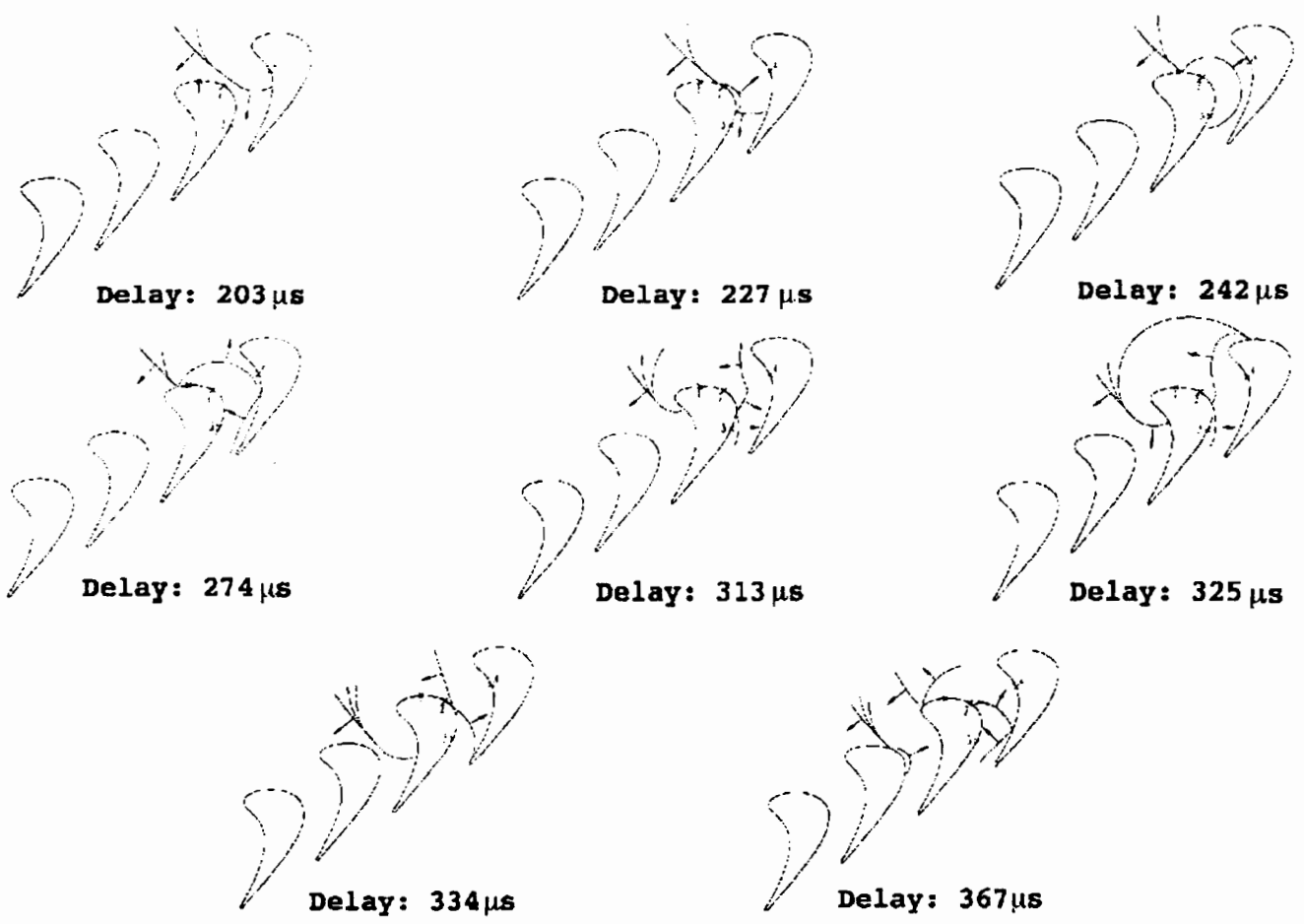

Figure 5.1: Rendering of Shock Passage Through Studied Blade Passage 
system. By channeling the output of the timing delay device back into the LeCroy, the $5 \mathrm{~V}$ delayed jump produced by the time delay circuit will also show up on the LeCroy. The time delay between the two spikes can easily be determined, Table 5.1. The manufacturer's calibration for the total temperature probe is used.

\subsubsection{Calibration of the Hotwire}

Calibration of the hot wire used for performing corrections to these calculated flow parameters was done in situ with a calibration performed for each ensemble average. This allowed a calibration without the uncertainty of a varying tunnel total temperature and pressure, as well as changes in the wire itself, between data sets. It is assumed that the wire calibration followed an existing experimental correlation so that only one point on the curve is sufficient to calibrate the wire.

Given average $P_{01}^{\prime}, T_{01}^{\prime}, P_{1}^{\prime}$ and the average hot wire voltage ahead of the shock, $V^{\prime}$, a calibration constant can easily be calculated. In the following development, the primes will be dropped from the equations, but all quantities are for the stationary reference frame. Using the following equation it is then possible to compute the flow Mach number at that point in the flow assuming $\gamma=1.4$.

$$
M_{1}=\sqrt{\frac{2}{\gamma-1}\left({\frac{P_{01}}{P_{1}}}^{\frac{\gamma-1}{\gamma}}-1\right)}
$$

Using the total pressure and the static pressure it is also possible using isentropic relations to calculate the temperature of the flow.

$$
T_{1}=T_{01}\left(\frac{P_{1}}{P_{01}}\right)^{\frac{\gamma-1}{\gamma}}
$$

then:

$$
U_{1}=M_{1} \sqrt{\gamma R T_{1}} .
$$

Given $T_{1}$ from above, a new ratio of specific heats, $\gamma$, is calculated and all quantities are recalculated. These new quantities are labeled with the subscript $c$. 


$$
C_{p}=1037.8-0.22371 T_{1}+2.8556 \times 10^{-4} T_{1}{ }^{2}+5.2694 \times 10^{-7} T_{1}^{3}-5.3327 \times 10_{-10} T_{1}^{4}
$$

$$
\gamma_{c}=\left[1-\frac{R}{C_{P}}\right]^{-1}
$$

then,

$$
\begin{gathered}
M_{1_{c}}=\sqrt{\left[\left(\frac{P_{01}}{P_{1}}\right)^{\frac{\gamma_{c}-1}{\gamma_{c}}}-1\right] \frac{2}{\gamma_{c}-1}} \\
T_{1_{c}}=T_{01}-\frac{U_{1}^{2}}{2 C_{p}} \\
U_{1_{c}}=M_{1_{c}} \sqrt{\gamma_{c} R T_{1_{c}}} \\
T_{m_{c}}=\frac{\left(T_{1_{c}}+T_{w}\right)}{2}
\end{gathered}
$$

where $T_{m}$ is the measured temperature of the wire.

Now,

$$
\begin{gathered}
\rho_{m_{c}}=\frac{P_{1}}{R T_{m_{c}}} \\
\mu_{m_{c}}=1.716 \times 10^{-5}\left(\frac{T_{m_{c}}}{273.16}\right)^{1.5}\left(\frac{383.716}{T_{m_{c}}+110.556}\right) \\
k_{m}=2.414 x 10^{-2}\left(\frac{T_{m_{c}}}{273.16}\right)^{1.5}\left(\frac{473.16}{T_{m_{c}}+200}\right) \\
R e^{*}=\left(\frac{\rho_{m_{c}} U_{1_{c}}}{\mu_{m_{c}}}\right)^{0.51} \\
\operatorname{Re}_{d}=\left(R e^{*}\right)^{1.961} d_{w}
\end{gathered}
$$

where $d_{w}$ is the hotwire diameter of $5 \mu m$. 


$$
N u^{*}=\frac{V^{2}}{k_{m_{c}}\left(T_{m_{c}}-T_{1_{c}}\right)}\left(\frac{T_{m_{c}}}{T_{1_{c}}}\right)^{-0.17}
$$

The relationship between $R e^{*}, N u^{*}$ and the calibration constant depends on the Reynolds number based on the tungsten wire diameter, $d_{w}$. If $R e_{d} \geq 44$, then

$$
C=\frac{R e^{*}}{N u^{*}}
$$

where $\mathrm{C}$ is the calibration coefficient.

Otherwise,

$$
C=\frac{\left(0.24+0.53 R e_{d}^{0.45}\right)}{N u^{*}}
$$

\subsection{Data Reduction}

\subsubsection{Upstream, Steady, Heated and Unheated Reference Density Fields}

As stated previously, seven static pressure data sets were recorded at each of the fifteen measurement locations. Each data set was averaged and then the averages of all five data sets were then averaged together to create a final average voltage value for all 15 upstream static pressures.

These voltage values were converted to average static pressures using the procedure discussed below.

$$
P_{a b s}=\frac{L C V}{(\operatorname{Sens})(G)}+P_{a t m}
$$

here $\mathrm{P}_{a b s}$ is the absolute pressure, $\mathrm{P}_{a t m}$ is the atmospheric pressure, LCV is the voltage read from the LeCroy (in $\mathrm{mV}$ ), Sens is the pressure transducer sensitivity (in $\frac{m V}{p s i}$ ) and $\mathrm{G}$ is the gain. It is difficult to obtain a calibration for the pressure transducers in such a dynamic system as a transonic wind tunnel. It is probably best to rely on the manufacturer's calibration curve. 
Using Eq. 2.1 these static pressures and the total pressures were used to calculate the Mach number of the flow over each of the taps. In order to normalize the results, the average upstream total pressures and temperatures were combined, Eq. 5.19, to produce an average upstream total density. Equation 5.20, showing the isentropic relations between pressure and temperature, was then used to calculate the average density during the unheated, steady runs at all 15 locations.

$$
\begin{gathered}
\rho_{0}=\frac{P_{0}}{R T_{0}} \\
\frac{P_{0}}{P}={\frac{T_{0}}{T}}^{\frac{\gamma}{\gamma-1}}
\end{gathered}
$$

The computer code avg01.cpp (Appendix B), written in $\mathrm{C}^{++}$was used in reducing the data from the upstream static pressure taps, the upstream total pressure tap and the upstream total temperature tap. Three basic types of averages are available in this program. The first type of average is called the "upstream average". This is a simple average, where the entire time record is added up and then divided by the total number of points. This average is used for all of the total pressure, total temperature, and upstream static pressure measurements in both the heated and unheated cases. The next type of average was not used directly in any data reduction, but was instead a component in the third type of averaging discussed. Here, a simple average was computed over three to five time records discretely at each time point. We refer to this type of average as an "unsteady average". The last type of averaging employed in reducing the steady density data is a combination of the unsteady and the upstream averaging technique. This type of averaging is used on both the heated and unheated steady static pressure wake data and is therefore referred to as a "wake average". The wake data, though completely recorded, will not be in this dissertation. A more sophisticated fringe detection algorithm than what is employed here for the upstream steady and unsteady interferograms is still in the development stage. 


\subsubsection{Upstream, Unsteady, Unheated Reference Density Fields}

An ensemble average, synchronized with the passing of the shock wave over the trigger Kulite transducer was used to smooth noise from the unsteady static pressure and hot wire signals. The program used to create the ensemble averages is called ensavg.cpp and is listed in Appendix C. The data reduction to produce the required time-accurate density fields proceeded as follows.

Using the static pressure traces, the strength of each shock is computed as it passed each tap. Given the shock strength, it was then possible to compute both the total pressure and total temperature and expected flow velocity behind each shock. It has been shown in benchtop tests, using two closely spaced Kulite transducers to calculate the shock velocity and strength, that the Kulite transducer (even the unscreened type, sometimes misses the peak pressure of the shock by up to $15 \%$. Therefore, some independent verification of the total temperature and total pressure changes is needed. The hot wire data was then used to correct the calculated flow parameters.

\section{Calculation of the Total Pressure and Temperature Variations}

In order to provide an initial guess for the total temperature and total pressure variations created by the passing of the initial and reflected shock waves, the Kulite static pressure traces were used in conjunction with the Rankine-Hugoniot equations modified for a moving shock wave. In making calculations with the modified RankineHugoniot equations, it is necessary to apply two separate reference frames, Fig. 5.2. One reference frame is stationary, where the shock wave is moving. All quantities related to this frame will be designated with a prime $\left({ }^{\prime}\right)$. The second frame is one which moves along at the speed of the shock, thus freezing the motion of the shock wave. All quantities in this reference frame will be unprimed.

First, the 15 ensemble averaged pressure traces were visually scanned, and the static pressure jumps, $\frac{P_{2}^{\prime}}{P_{1}^{\prime}}=\frac{P_{2}}{P_{1}}$, and the initial static pressures, $P_{1}^{\prime}=P_{1}$, and the shock passing times, $\mathrm{t}$, associated with these waves were recorded.

Given the initial total temperature, $T_{01}^{\prime}=T_{01}$, and the initial total pressure, 
Table 5.1: Time Delay Calibration

\begin{tabular}{|c||c|c|}
\hline Run & Timing Delay Setting & Actual Delay Time \\
\hline \hline 1 & $160 \mu s$ & $178 \mu s$ \\
\hline 2 & $140 \mu s$ & $258 \mu s$ \\
\hline 3 & $320 \mu s$ & $337 \mu s$ \\
\hline 4 & $377 \mu s$ & $393 \mu s$ \\
\hline 5 & $450 \mu s$ & $465 \mu s$ \\
\hline 6 & $500 \mu s$ & $518 \mu s$ \\
\hline
\end{tabular}

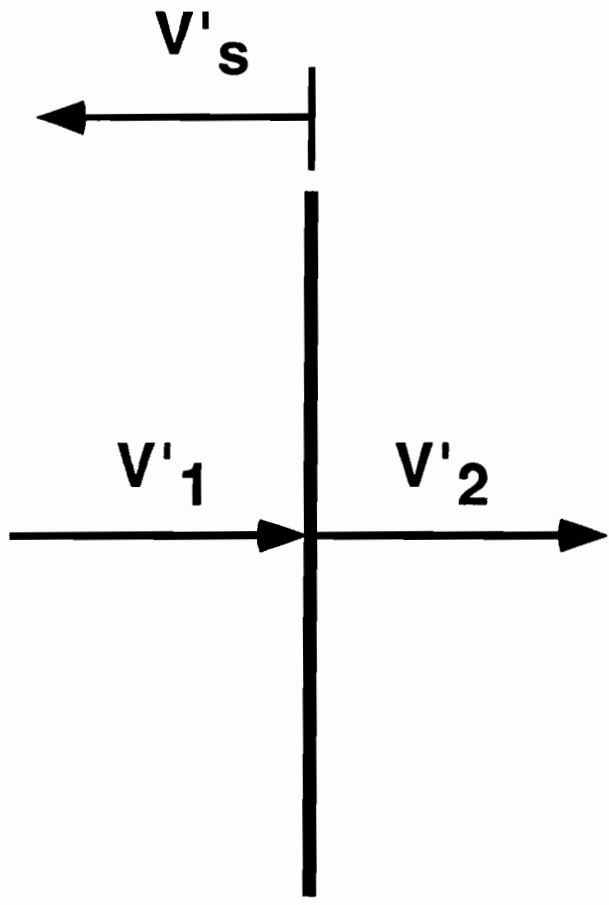

$1^{\prime} \quad 2^{\prime}$

Moving Wave

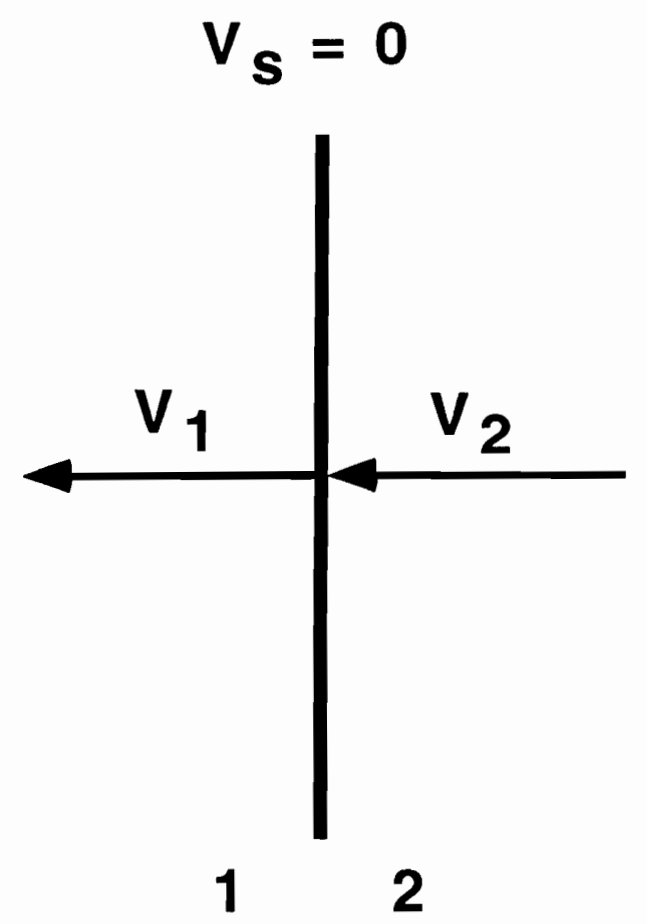

Stationary Wave

Figure 5.2: Properties Across a Moving Shock Wave 
$P_{01}^{\prime}=P_{01}$, the calculation of the approximate total temperature and pressure time history proceeds as follows:

1) Up until the first shock wave hits, the total temperature and pressure are recorded into the time history.

2) When the shock event occurs, the static pressure difference that was supplied from the static pressure traces is used to calculate the shock strength, $M_{s}$, using Eq. 5.21.

$$
M_{s}=M_{1}=\left[\frac{\gamma-1}{2 \gamma}+\frac{\gamma+1}{2 \gamma} \frac{P_{2}^{\prime}}{P_{1}^{\prime}}\right]^{\frac{1}{2}}
$$

The ratio of specific heats, $\gamma$, is assumed a constant 1.4 through all of the calculations since all of the data collection takes place before the shock tube contact surface passes through the test section.

3) Next, using the ratio of the initial static pressure, $P_{1}^{\prime}=P_{1}$, to the initial total pressure, $P_{01}^{\prime}$, the initial Mach number, $M_{1}^{\prime}$, is calculated using Eq. 5.22.

$$
M_{1}^{\prime}=\sqrt{\left[\left(\frac{P_{01}^{\prime}}{P_{1}^{\prime}}\right)^{\frac{\gamma-1}{\gamma}}-1\right] \frac{2}{\gamma-1}}
$$

Also, using this ratio and the isentropic relations, the initial static temperature, $T_{1}^{\prime}=T_{1}$, can be calculated from Eq. 5.23.

$$
T_{1}^{\prime}=T_{01}^{\prime}\left(\frac{P_{1}^{\prime}}{P_{01}^{\prime}}\right)^{\frac{\gamma-1}{\gamma}}
$$

4) The speed of sound ahead of the shock in the stationary reference frame is then given by Eq. 5.24

$$
a_{1}^{\prime}=\sqrt{\gamma R T_{1}^{\prime}}
$$

and the velocity ahead of the shock in the stationary reference frame, $V_{1}^{\prime}$, is then given by Eq. 5.25.

$$
V_{1}^{\prime}=M_{1}^{\prime} a_{1}^{\prime}
$$


5) Now, since $a_{1}^{\prime}=a_{1}$, the shock velocity in the stationary reference frame, $V_{s}^{\prime}$, is given by Eq. 5.26.

$$
M_{s}=\frac{V_{s}^{\prime}-V_{1}^{\prime}}{a_{1}} \text { therefore, } \quad V_{s}^{\prime}=M_{s} a_{1}+V_{1}^{\prime}
$$

6) Using the Rankine-Hugoniot relations, the speed of sound behind the shock wave, $a_{2}^{\prime}=a_{2}$, is given by Eq. 5.27.

$$
a_{2}^{\prime}=a_{2}=\sqrt{a_{1}{ }^{2} \frac{\left[2 \gamma M_{s}{ }^{2}-(\gamma-1)\right]\left[(\gamma-1) M_{s}^{2}+2\right]}{(\gamma-1)^{2} M_{s}^{2}}}
$$

7) The ratio of the two velocities, ahead of and behind the shock, in the moving reference frame is given by Eq. 5.28.

$$
\frac{V_{2}}{V_{1}}=\frac{(\gamma-1) M_{s}^{2}+2}{(\gamma+1) M_{s}^{2}}
$$

8) The velocity behind the shock in the stationary reference frame, $V_{2}^{\prime}$, is given in Eq. 5.29 .

$$
V_{2}^{\prime}=V_{s}^{\prime}-\left[\frac{V_{2}}{V_{1}} \frac{a_{2}}{a_{1}}\left(V_{s}^{\prime}-V_{1}^{\prime}\right)\right]
$$

9) Using the speed of sound, $a_{2}$, the static temperature behind the shock, $T_{2}^{\prime}$, can now be computed using Eq. 5.30.

$$
T_{2}^{\prime}=\frac{a_{2}{ }^{2}}{\gamma R}
$$

10) The Mach number in the stationary reference frame behind the shock, $M_{2}^{\prime}$, is (Eq. $5.31)$.

$$
M_{2}^{\prime}=\frac{V_{2}^{\prime}}{a_{2}}
$$

11) Equations 5.32 and 5.33 can be used to calculate the new total temperature, $T_{02}^{\prime}$, and total pressure, $P_{02}^{\prime}$, behind the shock wave.

$$
T_{02}^{\prime}=T_{2}^{\prime}\left(1+\frac{\gamma-1}{2}{M_{2}^{\prime 2}}^{2}\right)
$$




$$
P_{02}^{\prime}=P_{1}^{\prime}\left(\frac{P_{2}^{\prime}}{P_{1}^{\prime}}\right)\left(\frac{T_{02}^{\prime}}{T_{1}^{\prime}}\right)^{\frac{\gamma}{\gamma-1}}
$$

12) Now, $P_{02}^{\prime}$ is set as the new $P_{01}^{\prime}$ and $T_{02}^{\prime}$ is set as the new $T_{01}^{\prime}$ and the procedure is repeated until the end of the data is reached.

\subsubsection{Unsteady Density Data Correction}

Using a high overheat ratio (1.8) reduces the sensitivity of the hot wire to variations in total temperature. Since the passing shock wave and reflected shocks are weak (peak pressure ratio of 1.2 ), hot wire voltage variations due to the fluctuating total temperature are neglected [40].

Using the pressure trace from the Kulite transducer and the approximate total pressure, $P_{0}$, and total temperature, $T_{0}$, traces calculated using moving shock theory, the approximate flow temperature and velocity traces can be calculated using Eqs. $5.1,5.2$ and 5.7 .

Proceeding as in the hot wire calibration procedure (point by point), a new specific heat at constant pressure, $C_{p}$, is found. As before, this new $C_{p}$ is then used in Eqs. 5.5 to 5.12 and Eq. 5.15 to find $N u^{*}$. Depending upon the $R e_{d}$ obtained during calibration, either Eq. 5.16 or Eq. 5.17 is used to calculate $R e^{*}$ given the calculated calibration constant.

Using Eq. 5.14 the Reynolds number based on the wire diameter is found and subsequently used to calculate a new velocity, $U_{1}$ (Eq. 5.34) and a new approximate static temperature, $T_{1}$ (Eq. 5.2).

$$
R e_{d}=\frac{\rho_{m_{c}} U_{1} d_{w}}{\mu_{m_{c}}}
$$

The new $T_{1}$ is compared against the old $T_{1}$. If the convergence criteria is reached, then the new $T_{1}$ is kept as the temperature and a density is calculated using the following equation:

$$
\rho_{1}=\frac{P_{1}}{R T_{1}} .
$$


Otherwise, the new static temperature becomes the old static temperature and the calculations are performed again. This procedure is repeated, point by point, until convergence is obtained or the correction significantly increases from the previous step (significantly meaning more than $20 \%$ of the total density value) giving an error message. Figures 5.3 through 5.17 show plots of both the uncorrected and corrected data. It should be noted that the signals show excellent repeatability, since the hot wire and pressure data were taken up to two weeks apart.

\subsection{Digital Image Processing}

In an effort to automate the image data reduction process, various digital image processing techniques were investigated. Previously, the steady state fringe detection had been performed manually by digitizing the fringes using a tablet. However, because of the large number of images to be reduced, it was decided the fringe detection needed to be automated.

Five $C^{++}$codes were written to reduce the gray-scale images to density values. The first of these codes, referred to as FringeFinal.cpp (Appendix E), has four main functions: image stretching, low-pass filtering, adaptive binarization and line thinning. The second code, called sitc (Appendix F), is used to shift the images so that each type of image in each zone is centered on a specific reference point. Next, the code flip (Appendix G), mirrors the images about the central reference point. Finally, the code ProcFin.cpp (Appendix H), performs fringe counting and outputs the final reduced density values.

\subsubsection{Stretching}

The $512 \times 480$ pixels in the CCD camera were arranged in a square grid, thus the aspect ratio of each pixel was not the 1:1 aspect ratio required by the data reduction program. Therefore, a stretching algorithm was employed to expand the distorted horizontal axis by a factor of 1.21 . This algorithm consists of two nested loops that traverse the destination image area pixel-by-pixel. For each pixel in the destination 


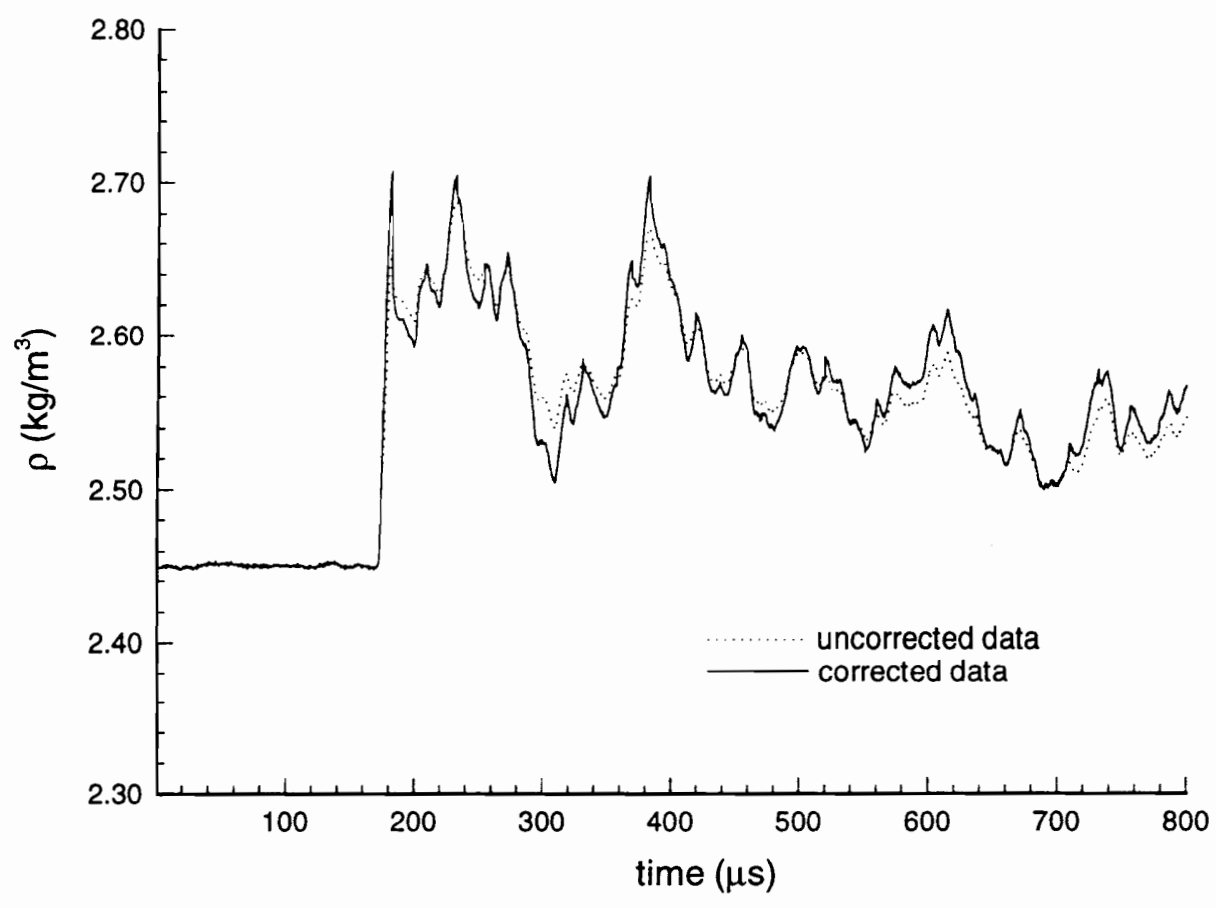

Figure 5.3: Hot Wire Corrected Unsteady Density Trace, Hole \#1 


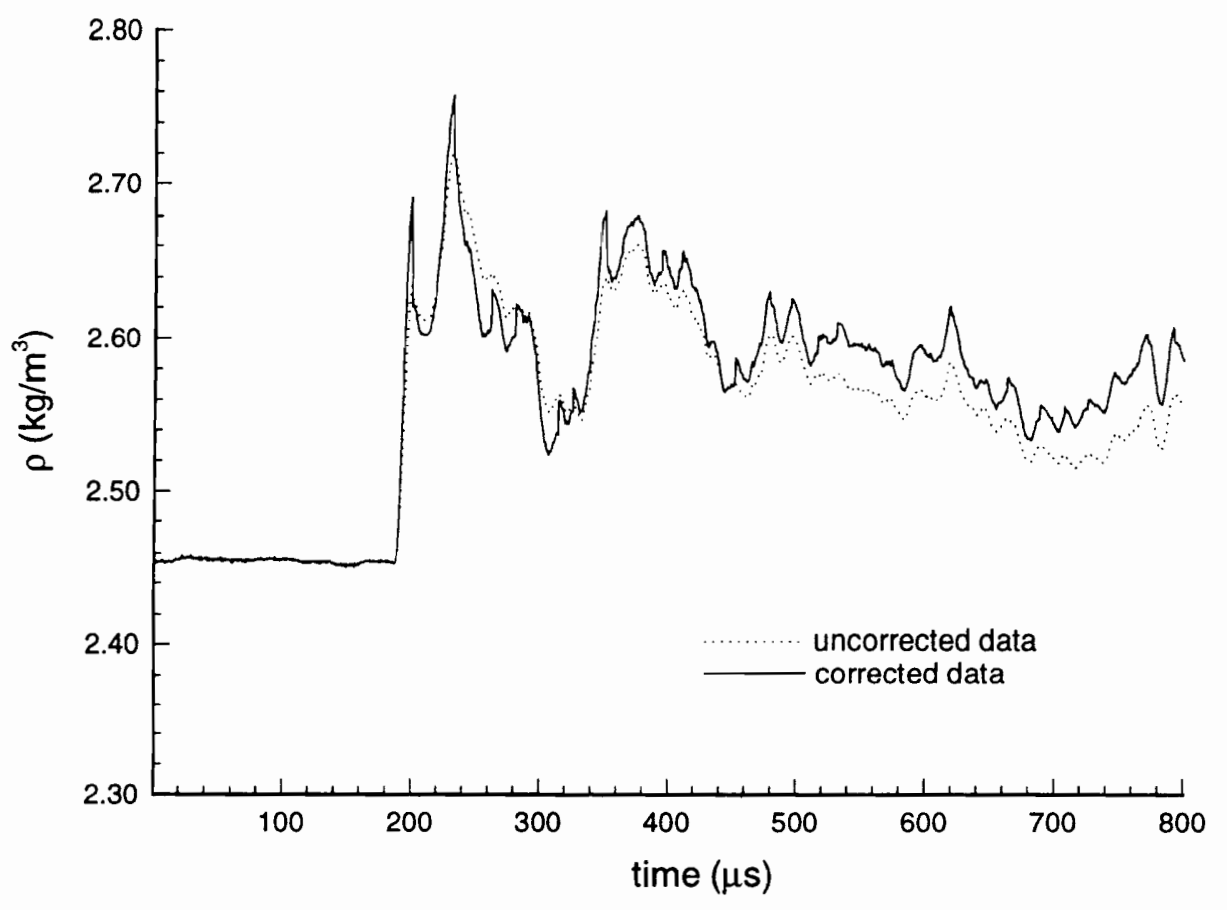

Figure 5.4: Hot Wire Corrected Unsteady Density Trace, Hole \#2 


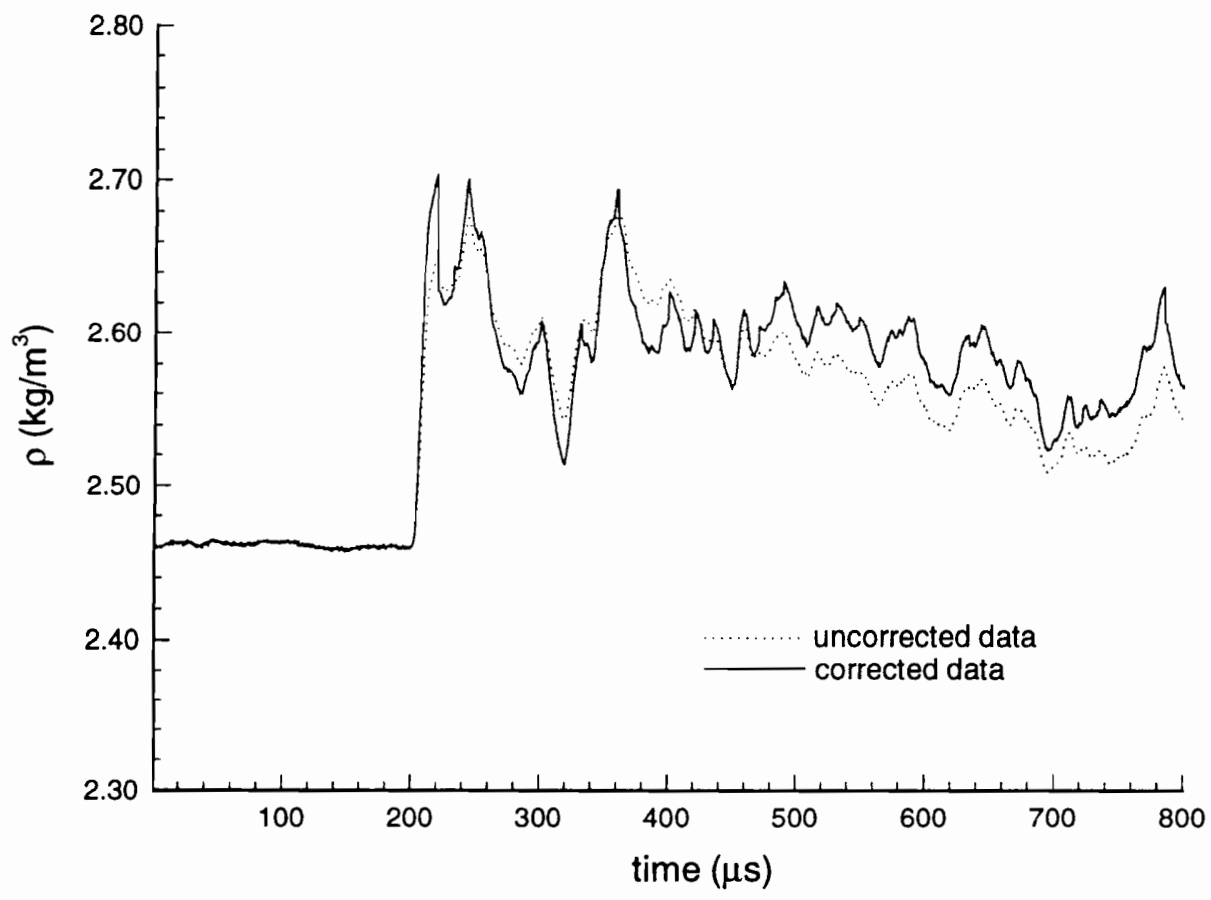

Figure 5.5: Hot Wire Corrected Unsteady Density Trace, Hole \#3 


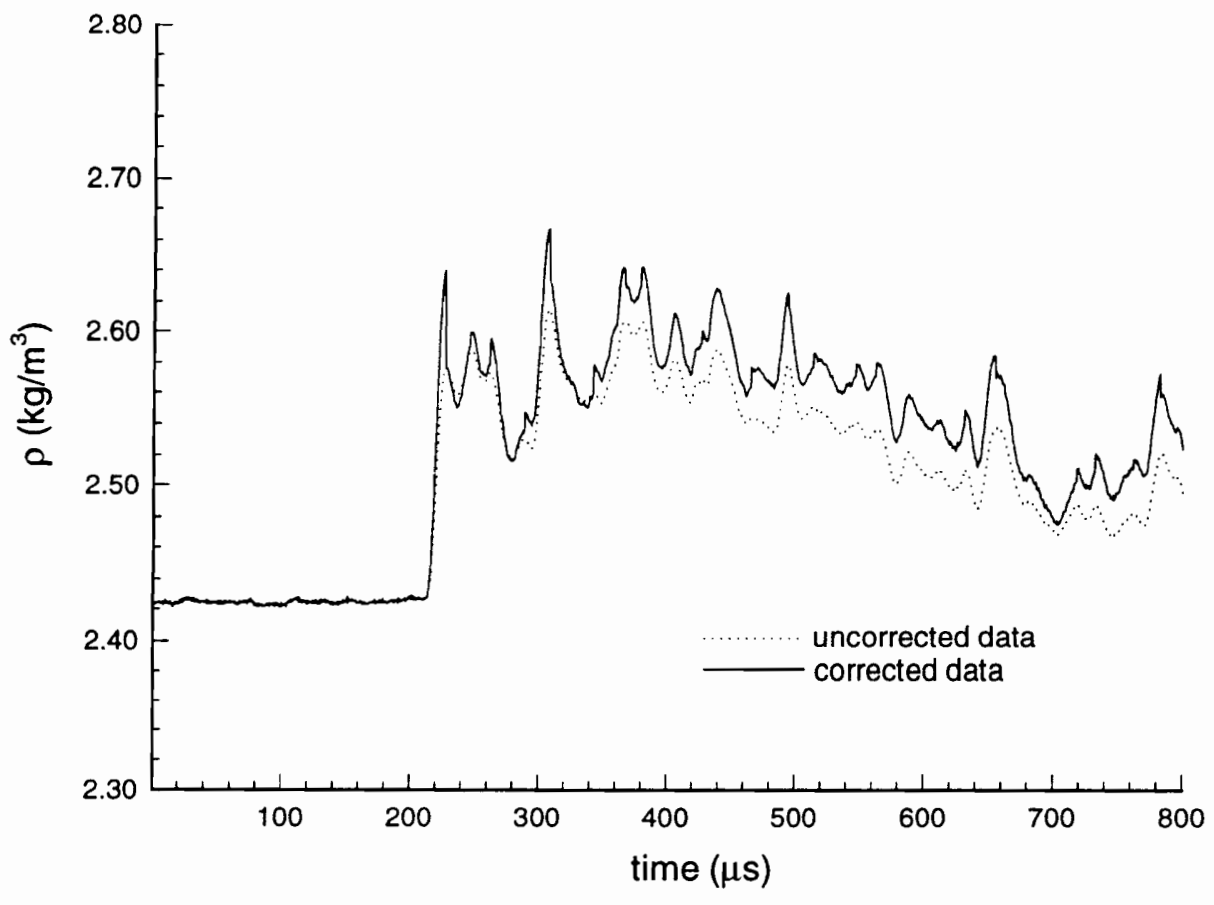

Figure 5.6: Hot Wire Corrected Unsteady Density Trace, Hole \#4 


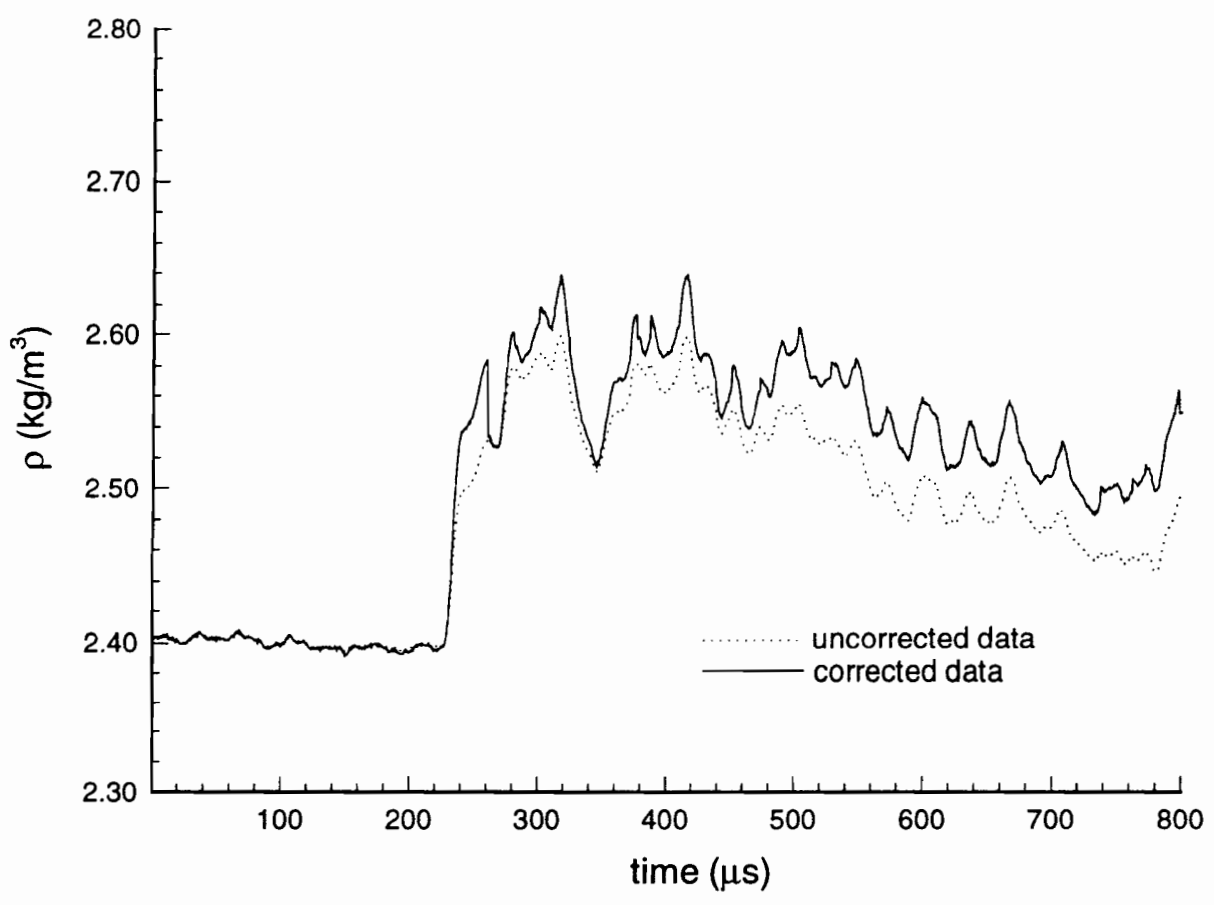

Figure 5.7: Hot Wire Corrected Unsteady Density Trace, Hole \#5 


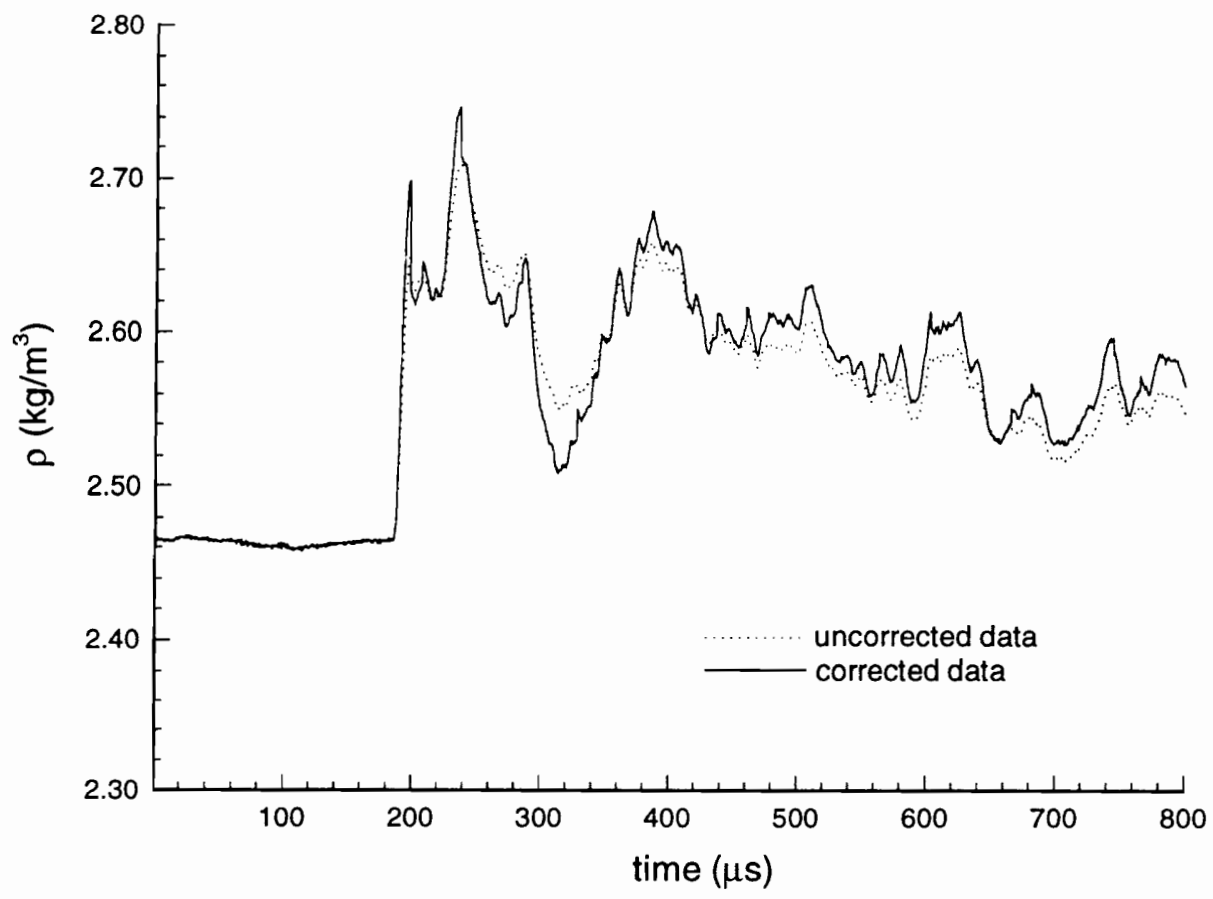

Figure 5.8: Hot Wire Corrected Unsteady Density Trace, Hole \#6 


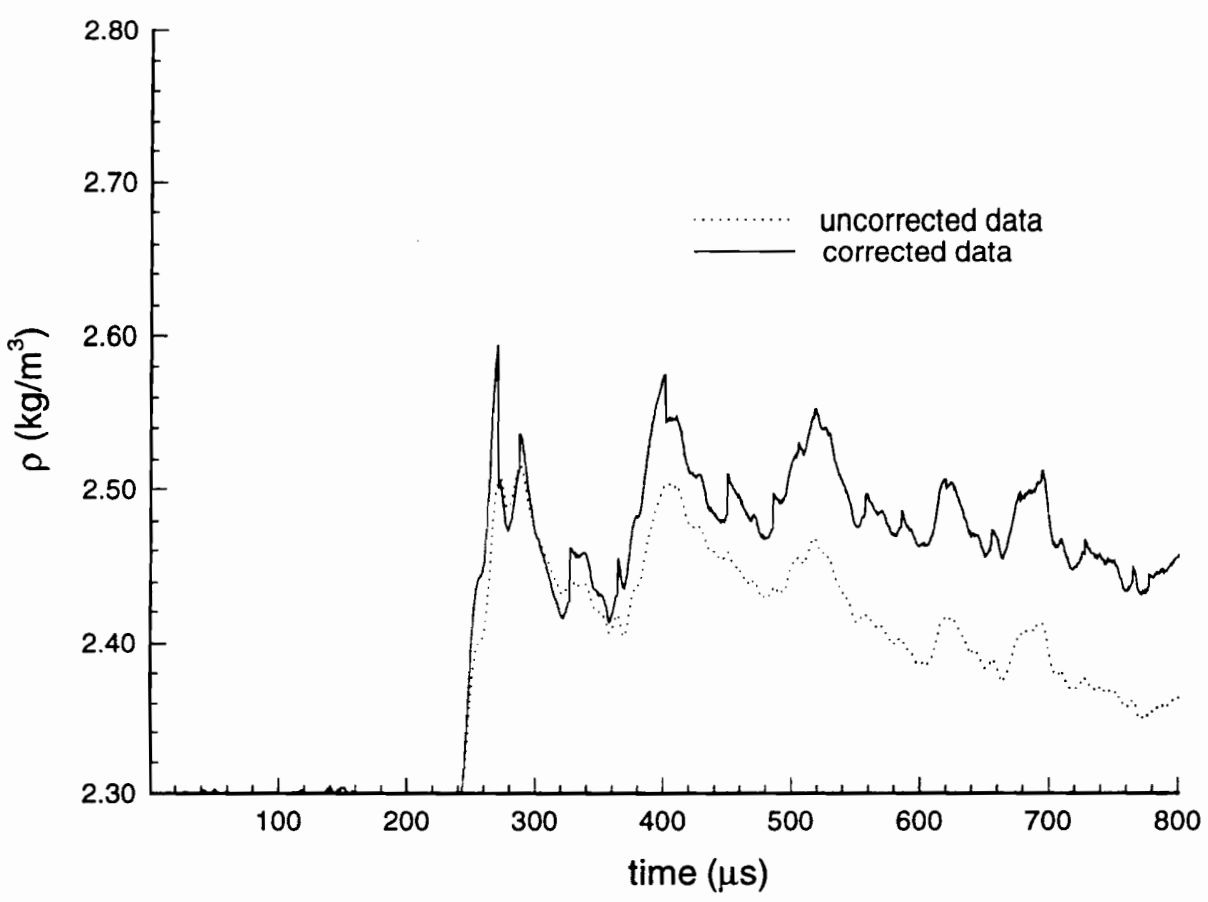

Figure 5.9: Hot Wire Corrected Unsteady Density Trace, Hole \#7 


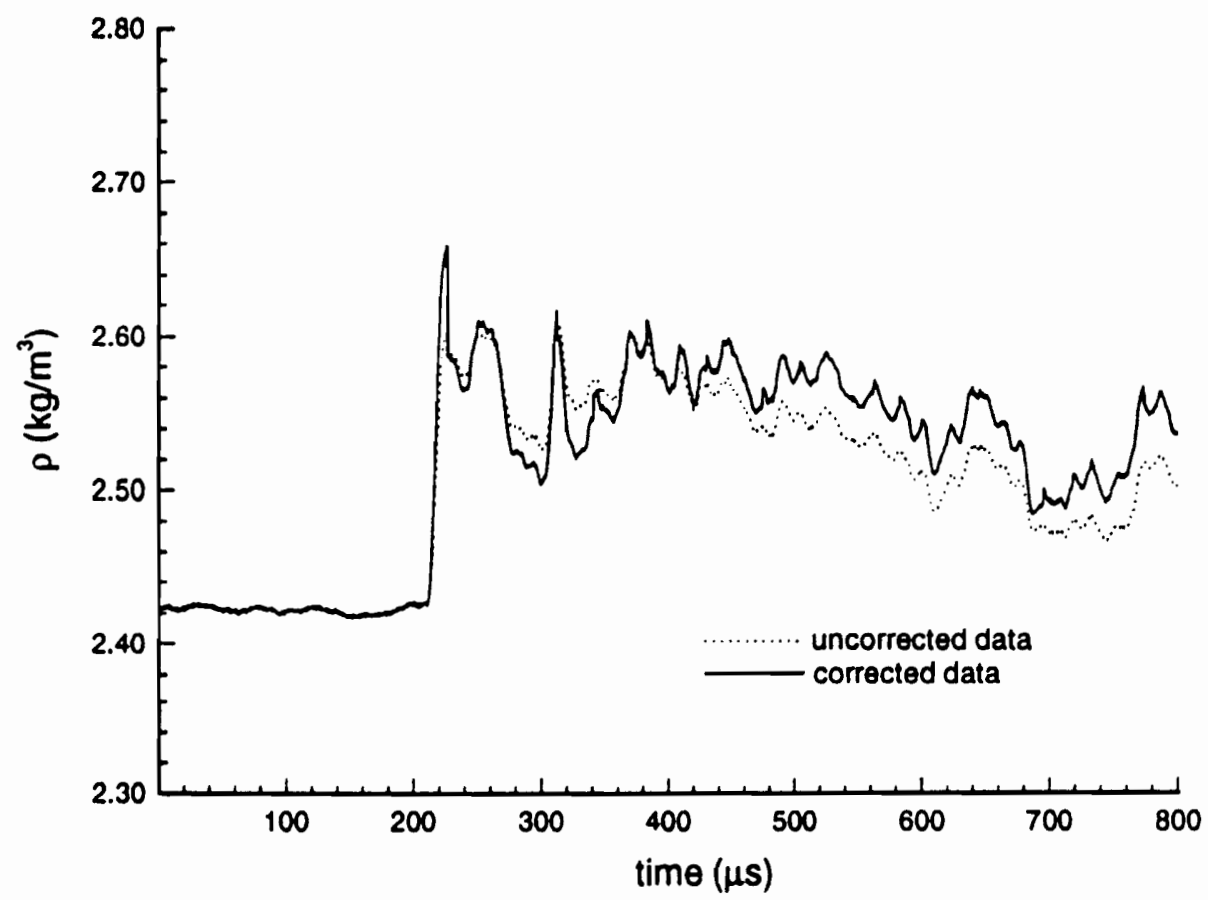

Figure 5.10: Hot Wire Corrected Unsteady Density Trace, Hole \#8 


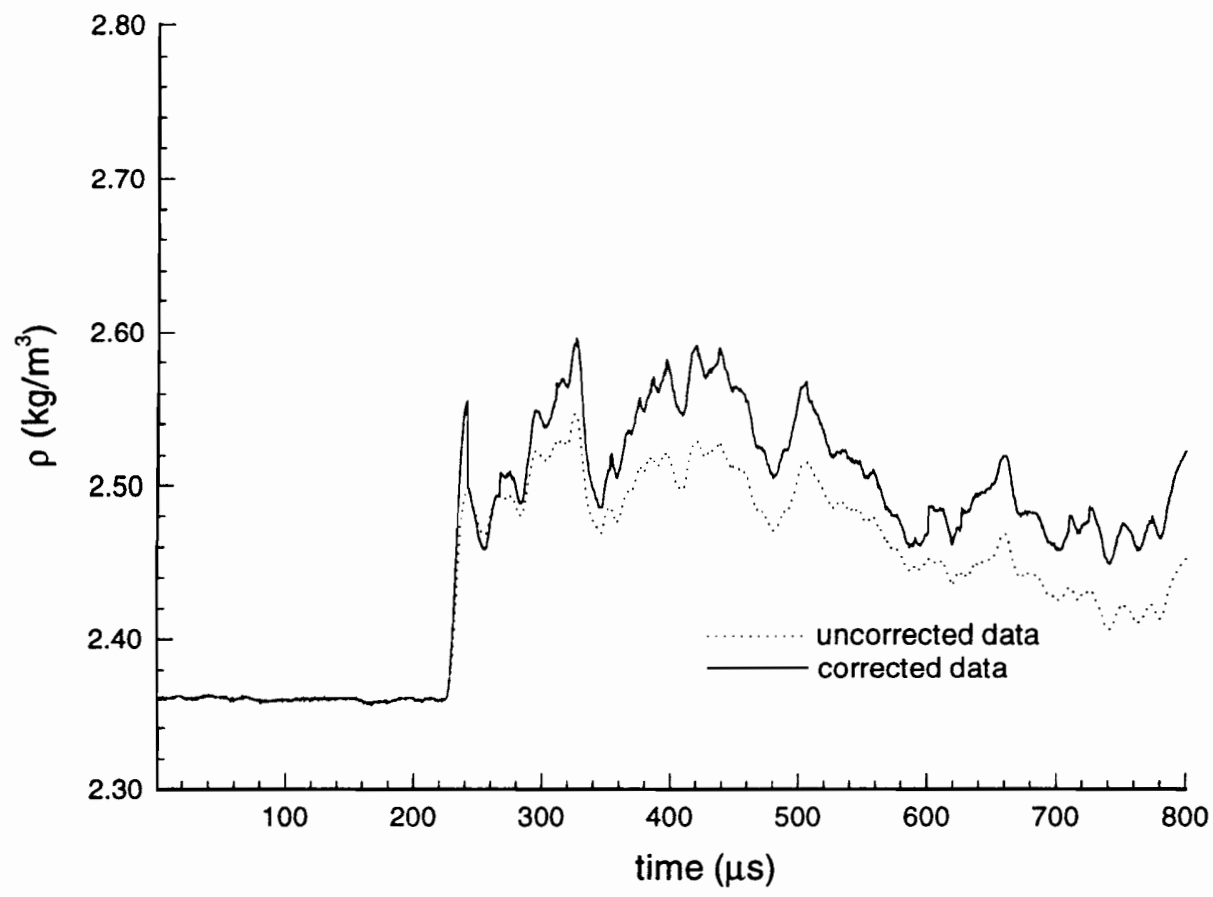

Figure 5.11: Hot Wire Corrected Unsteady Density Trace, Hole \#9 


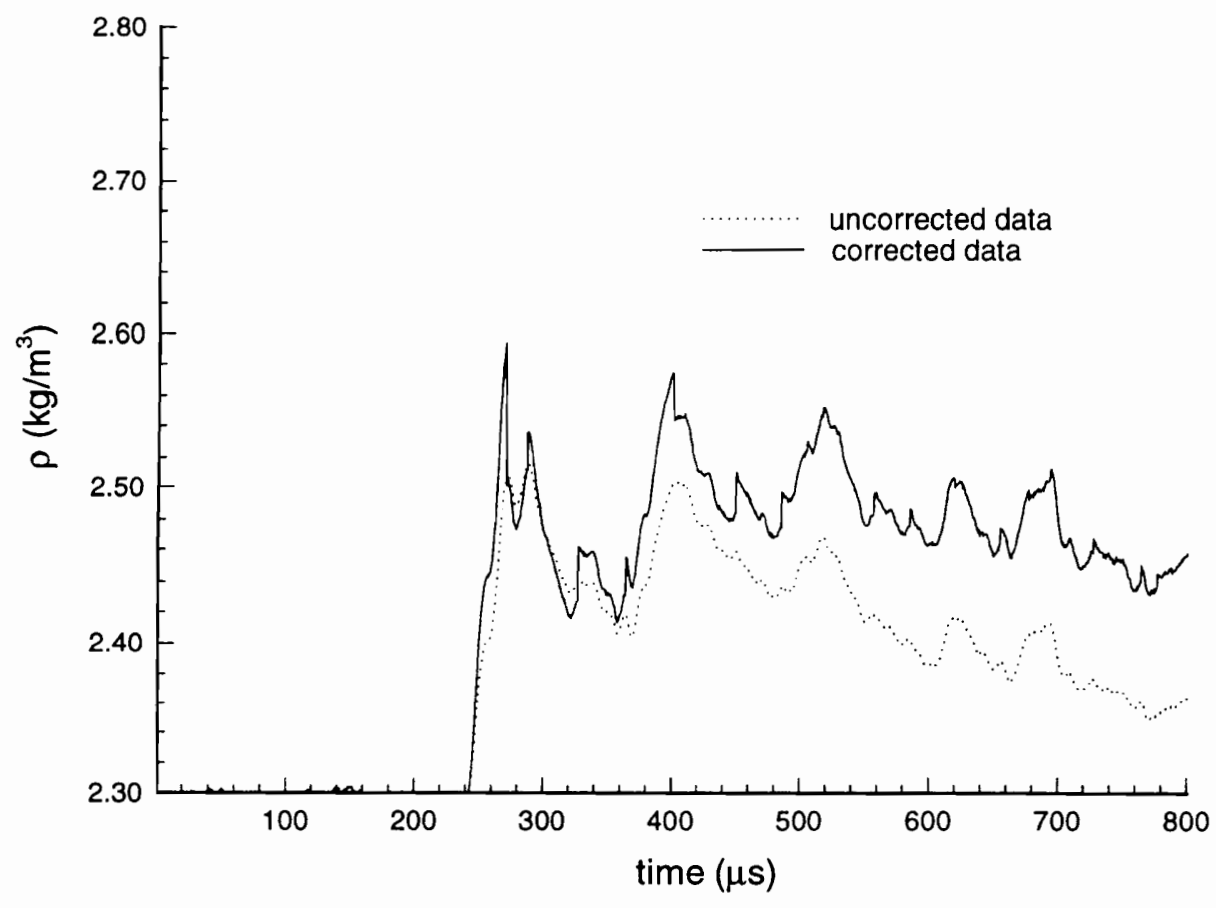

Figure 5.12: Hot Wire Corrected Unsteady Density Trace, Hole \#10 


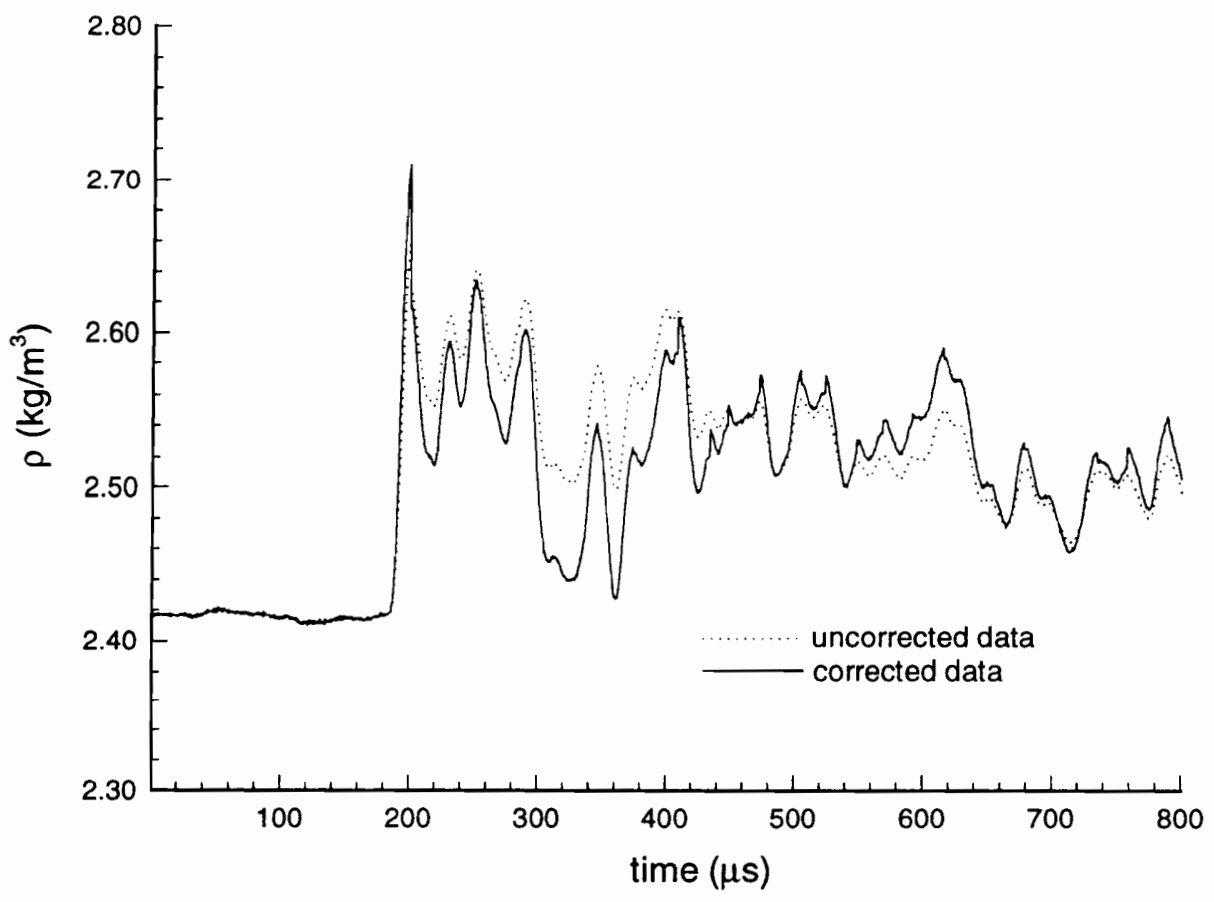

Figure 5.13: Hot Wire Corrected Unsteady Density Trace, Hole \#11 


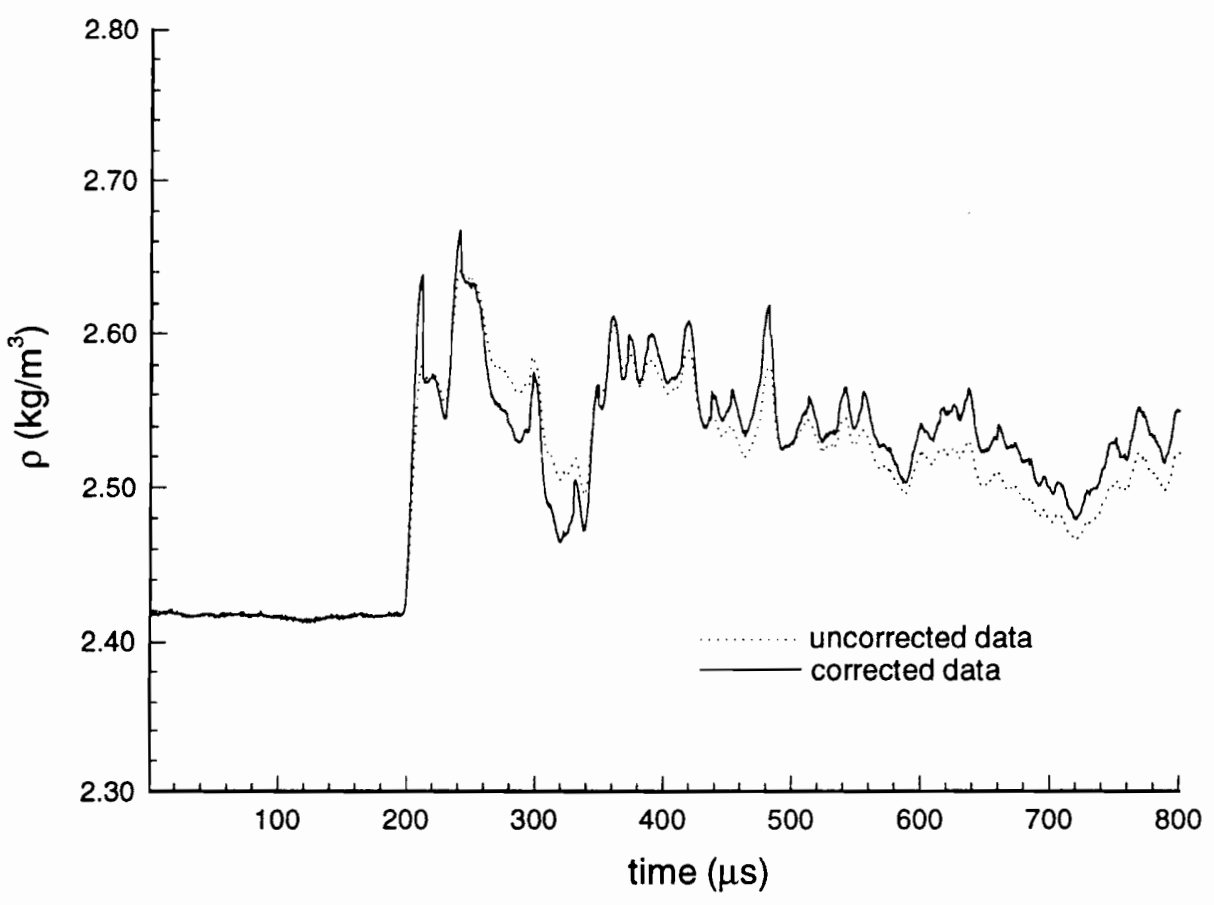

Figure 5.14: Hot Wire Corrected Unsteady Density Trace, Hole \#12 


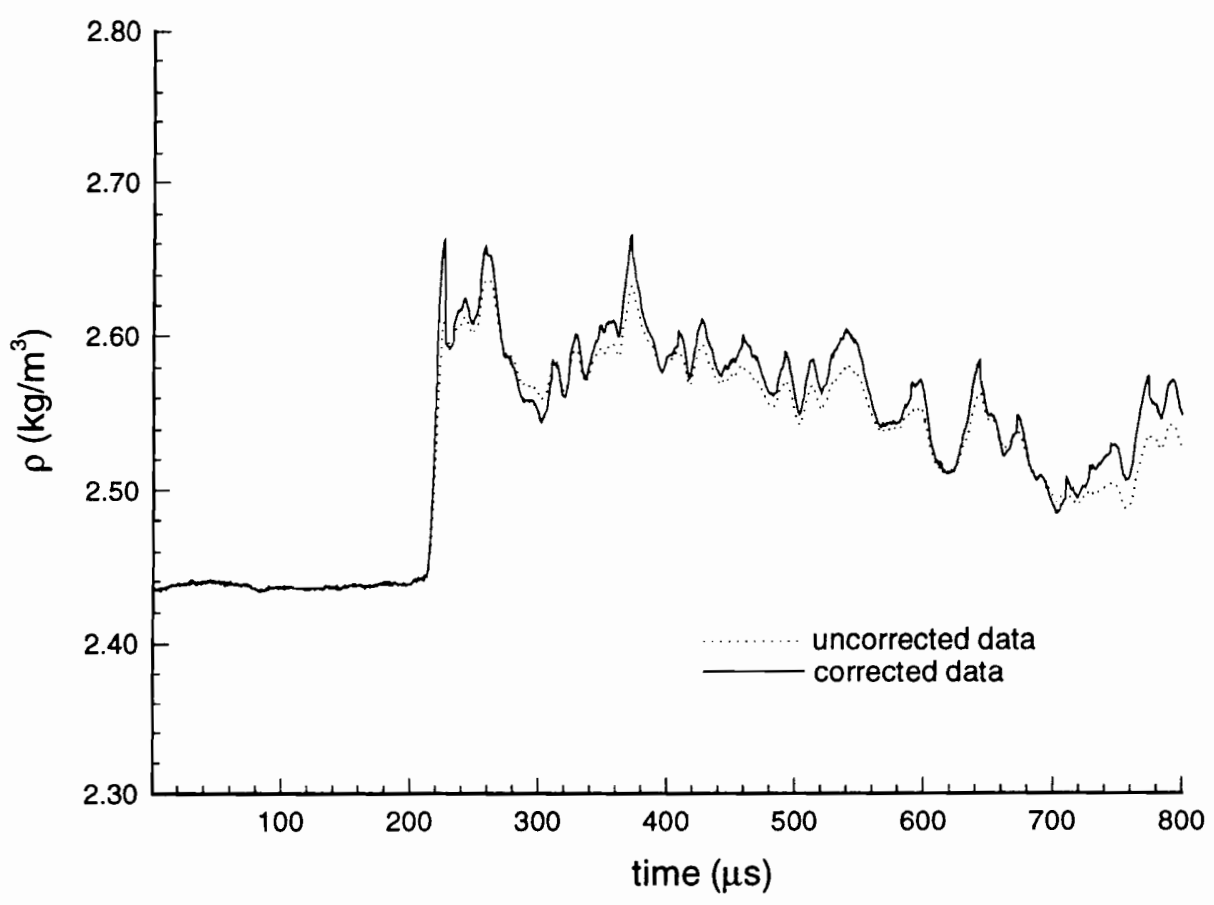

Figure 5.15: Hot Wire Corrected Unsteady Density Trace, Hole \#13 


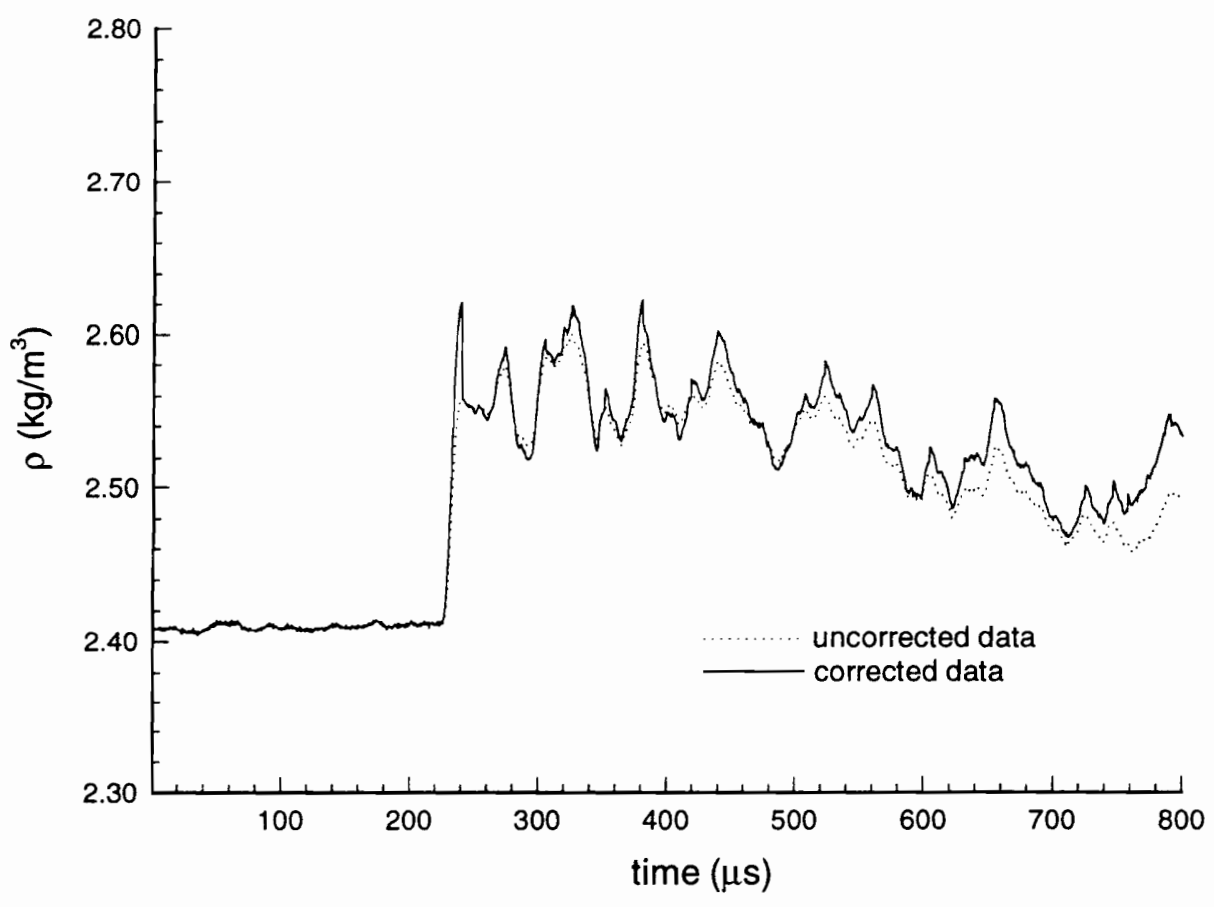

Figure 5.16: Hot Wire Corrected Unsteady Density Trace, Hole \#14 


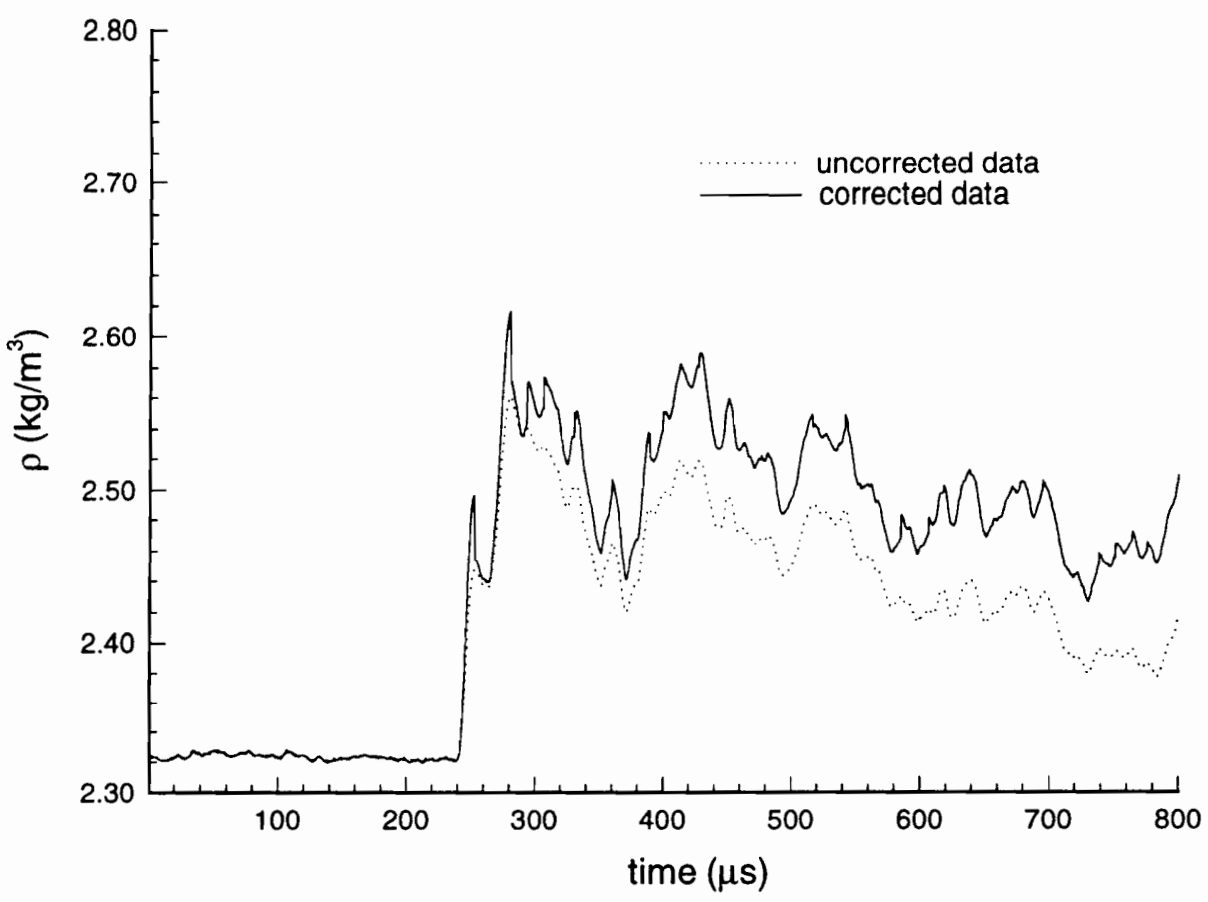

Figure 5.17: Hot Wire Corrected Unsteady Density Trace, Hole \#15 
image, a reverse mapping is used to determine the corresponding pixel in the source image. Often this pixel address is a fractional value. This means that the calculated address would have fractional components of the neighboring pixels. Here, an interpolation technique is used. The four pixels that surround the calculated position of the destination pixel in the source image are fetched and a linear interpolation is used on pixel intensities to determine the destination pixel's gray-scale intensity value.

\subsubsection{Low-Pass Filtering}

In order to reduce the presence of artifacts in the image introduced by the CCD camera and the frame grabber, it was necessary to spatially filter the image. A lowpass filter was used, taking great care in setting the upper frequency limit, to remove these artifacts without destroying any fringe details. Low-pass filters are frequently employed in image processing to reduce visual noise [41].

\subsubsection{Adaptive Binarization}

The image information output from a CCD camera is in a format known as gray scale. Gray scale images utilize between two and eight bits per pixel, which results in 4 to 256 levels of gray available in an image. In this case, the CCD camera returns an eight bit gray scale image, providing 256 levels of gray. The image processing techniques already employed required gray scale images for proper processing. However, in order to detect the fringe edges and to enhance fringes that are lost in uneven background lighting, adaptive binarization (also referred to as adaptive thresholding) must be employed. In fixed thresholding, a single gray level is set as the cutoff level. Any pixel values falling under this level are set to 0 (black). Otherwise the pixel value is set to 1 (white), producing a simple black and white image. Setting a single threshold level for the interferometric images is difficult, since the image is disturbed by uneven lighting. Thus, a threshold level that adapts itself to the local gray scale level is required. Locally adaptive binarization methods compute a threshold level for each pixel in the image on the basis of the information contained in the neighborhood of that pixel. The method employed here uses the idea of a threshold surface. If a pixel 
$(\mathrm{x}, \mathrm{y})$ in the input image has a higher gray level than the threshold surface evaluated at $(\mathrm{x}, \mathrm{y})$, then the pixel is labeled as background, otherwise it is labeled as an object (black). Our threshold surface consists of a 20 by 20 pixel block surrounding the studied pixel $(\mathrm{x}, \mathrm{y})$. The average gray level is computed in the $20 \mathrm{x} 20$ threshold surface and set as the threshold level for the surface [42]. Figure 5.18 shows an example of an adaptively binarized image.

\subsubsection{Line Thinning}

Since the binarization algorithm can leave extremely thick fringes that could complicate the fringe counting, it was necessary to process the binarized images with a line thinning algorithm. The Hilditch algorithm was chosen for its robust nature and ease of implementation [43]. Figure 5.19 shows an example of a thinned image.

\subsection{Uncertainties}

A FORTRAN jitter program was developed in order to determine the required experimental uncertainties for each measured quantity to produce an target error in the density $\leq \pm 5 \%$. It was found, however that a jitter program is more useful in determining the final uncertainty given the experimental uncertainties. Thus, an estimate of the experimental uncertainty of each measured quantity needed to be determined.

First, the sample rates and data storage requirements for the LeCroy waveform recorder needed to be determined. A sample rate of 2 million samples/sec was used, providing a $0.5 \mu$ s temporal resolution. A gain of 100 is provided by the amplifier; a maximum voltage of $5 \mathrm{~V}$ is expected. Using a $10 \mathrm{~V}$ maximum voltage range with a -5 $\mathrm{V}$ offset gives a resolution of $\pm 2.5 \mathrm{mV}$ by the $\mathrm{A} / \mathrm{D}$ board. Also, since each module of the LeCroy has $1 \mathrm{Mb}$ of memory, 0.125 seconds of data can be taken on all four channels at a sample rate of $2 \mathrm{M}$ samples/second.

First, the uncertainty of the pressure data (in $\mathrm{mV}$ ) is computed. These are computed with the amplifier gain factored in. The linearity and hysteresis of the Kulite transducers should produce an uncertainty of $\pm 50.0 \mathrm{mV}$. The thermal sensitivity 


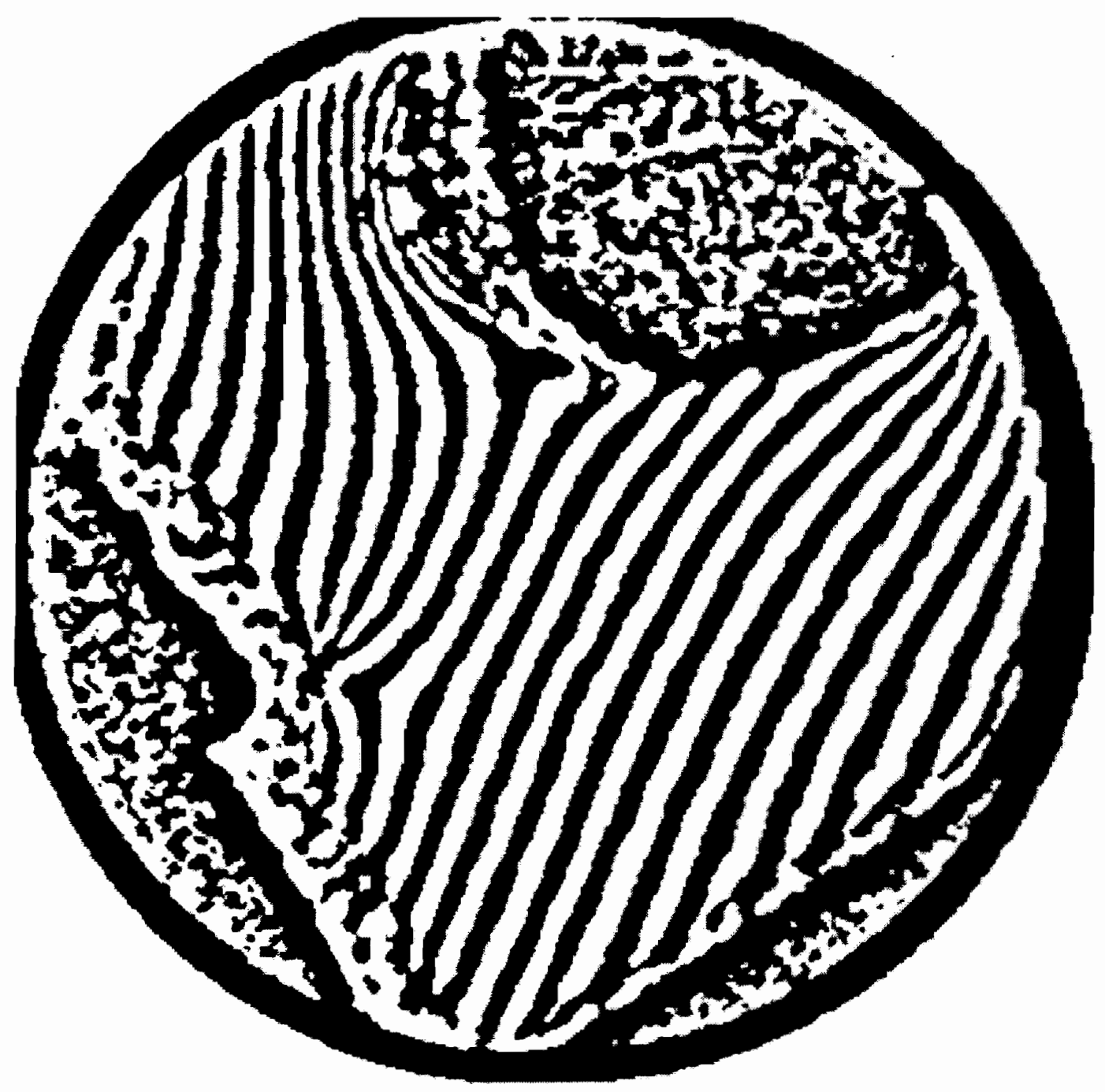

Figure 5.18: Binarized Interferogram of Unheated Flow in Area 1 


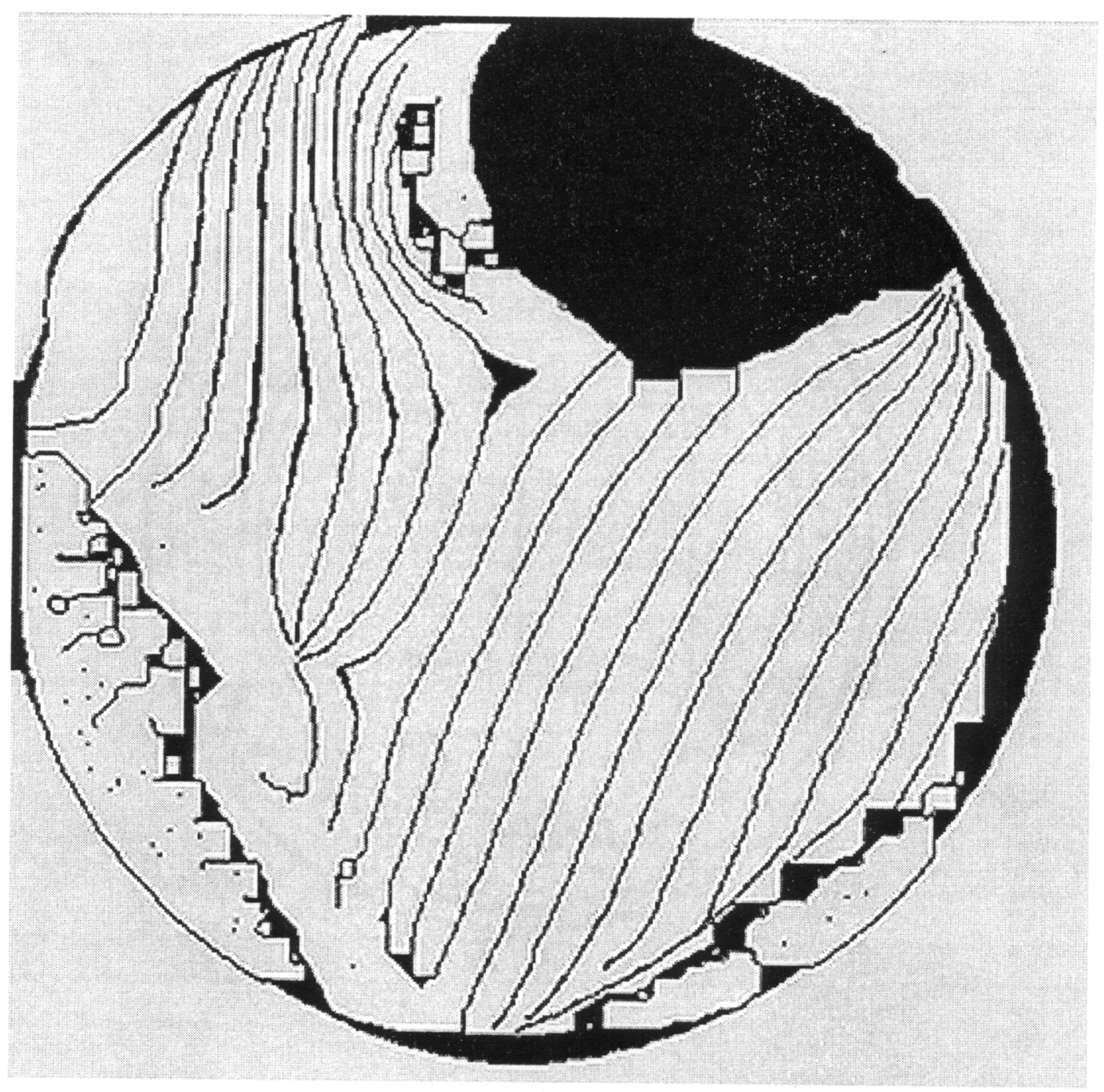

Figure 5.19: Thinned Interferogram of Unheated Flow in Area 1 
shift over $10^{\circ} \mathrm{F}$ should contribute an uncertainty of $\pm 50.0 \mathrm{mV}$, and the repeatability contributes $\pm 10 \mathrm{mV}$ to the uncertainty. Therefore, the overall uncertainty in the raw pressure measurement is $\pm 71.5 \mathrm{mV}$. The pressure tap locations are believed to be known to within $\pm 0.0254 \mathrm{~mm}$. The total temperature is known to $\pm 1.0 \mathrm{~K}$.

Since the HeNe laser was chosen over the Argon laser, the problems associated with temperature induced frequency drift are greatly reduced. This is because, in general, the HeNe bore is a fraction of the length of a typical Argon laser bore, thus the positioning of the lasers mirrors is not as critical. The HeNe laser uses an internal mirror arrangement, less complex than the external Brewster window arrangement employed by most gas lasers. The internal dielectric mirror arrangement of $\mathrm{HeNe}$ laser has the two mirrors cemented directly to the ends of the discharge tube. The alignment of these mirrors, which is critical to the achievement of a high power output, is performed before the cement sets. This method has the advantage that the laser is prealigned and cannot be misaligned without bending or breaking the discharge tube.

The uncertainty in the fringe count is the most difficult parameter to determine. Since a $604 \times 576$ pixel CCD camera is used, the spatial resolution of the camera for a $5.59 \times 5.59 \mathrm{~cm}(2.2 \times 2.2$ in $)$ object should be $\pm 0.093 \mathrm{~mm}$. The trigger jitter also needs to be taken into account. $\mathrm{A} \pm 20 \mu \mathrm{s}$ jitter time will produce $\mathrm{a} \pm 7.2 \times 10^{-6}$ $m$ uncertainty in the fringe location. This produces an overall fringe resolution of \pm $0.093 \mathrm{~mm}$. Since there is nominally a fringe every $2.286 \mathrm{~mm}$ and the fringe count is nominally 2.33 , the number of fringes for any given density computation is believed to be known with \pm 0.1 uncertainty.

Another uncertainty parameter that is difficult to estimate is the uncertainty in the reference density field. Using the given uncertainty for the Kulite pressure transducers of $\pm 71.5 \mathrm{mV}$ and estimating, based on the allowable error in the iterative stopping criteria and the estimated probe interference on the passing shock flowfield, the estimated error of the density at the 15 measurement points is $\pm 0.01 \frac{\mathrm{kg}}{\mathrm{m}^{3}}$ or \pm $0.35 \%$.

Using the uncertainty values above as the jitter program input and values representative of typical data (without a shock directly over the reference domain), an overall uncertainty value was found for the computed densities. By approximating 
the effects of the interpolation on the uncertainties of the reference pressure values, a simplified analytical analysis was performed. The values from both calculations compared well, lending validity to the jitter program formulation. The computed density value and overall uncertainty given by the jitter program was $2.808 \pm 0.093$ $( \pm 3.31 \%) \mathrm{kg} / \mathrm{m}^{3}$. This uncertainty is well within acceptable levels. 


\section{Chapter 6}

\section{Results}

Figures 6.1 through 6.8 shows all of the steady and unsteady raw interferograms. Figures 6.1 and 6.2 show the steady, adiabatic interferograms. It was necessary to use two separate images because the entire flowfield would not fit in a single image. Of note is the presence of the double image mentioned in previous sections. The double image is most noticeable for the blades where one sees a black image and a second gray image displaced from the first. In order to reduce the interferograms, both a no flow (an interferogram taken with out any tunnel flow) and a flow image must be captured. In a no flow interferogram, the density is constant, therefore, the fringes are basically strait lines. When the cascade flowfield is generated and a flow image captured, the density variations shift the fringes. This phenomena is especially noticeable near the crown and the stagnation points of the steady interferograms. Any shock waves present in the flowfield are characterized by the coalescence of a large number of fringe lines, as can be seen on the right hand side of Figure 6.6. Figures 6.3 and 6.4 show the heated flow interferograms. Figures 6.5 through 6.10 give the unsteady interferograms for area 1 at the delay times detailed in Table 5.1. The unsteady interferograms for area 2 are given in Figs. 6.11 through 6.16. Figure 6.17 shows the positions of area 1 and area 2 relative to the studied blade passage.

The raw data is converted to a density field using the procedure outlined in Section 5.2. The reduced density field plots are presented below. First the steady data will be introduced, and then the unsteady density field results will be discussed. 


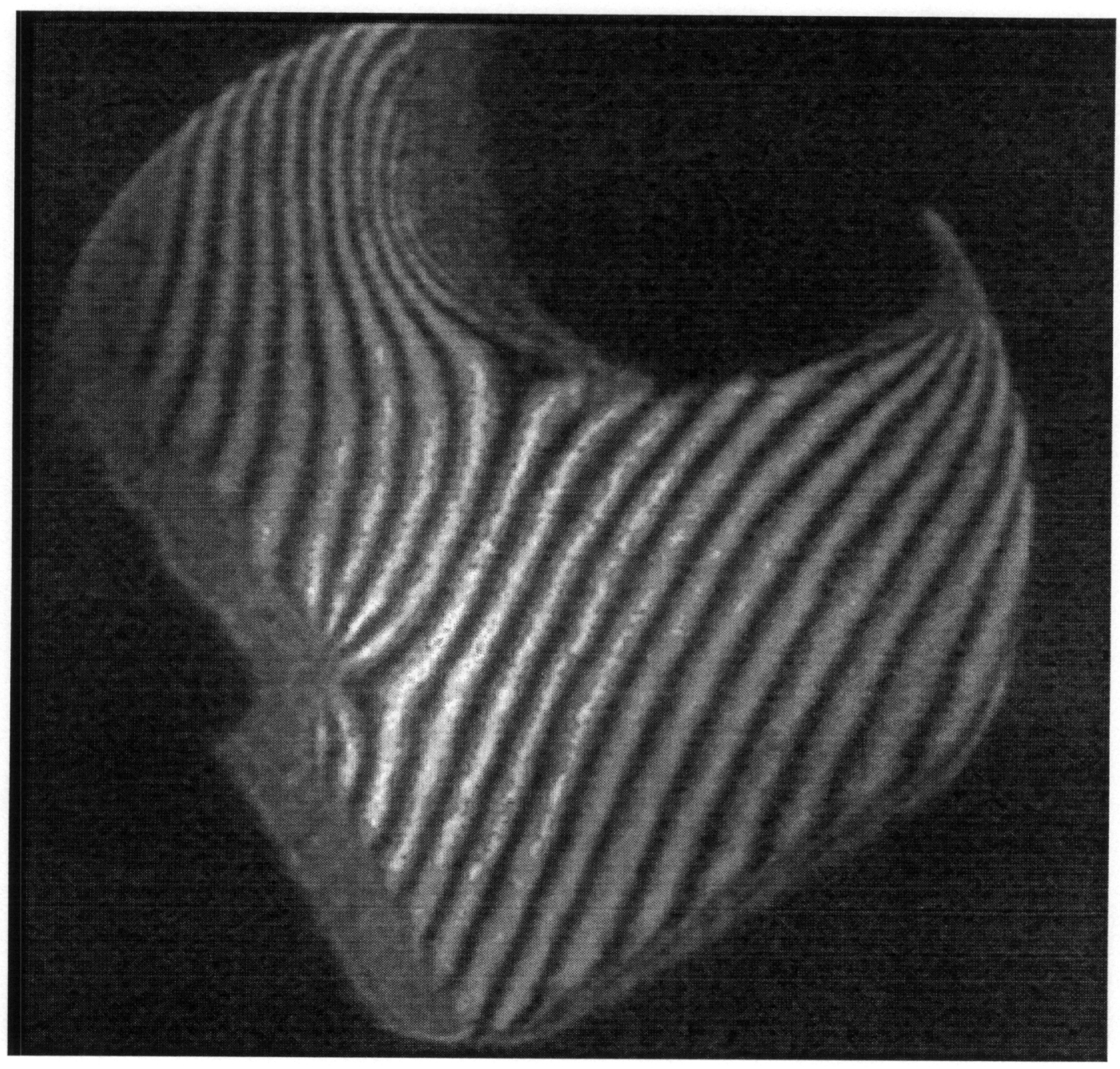

Figure 6.1: Interferogram of Unheated Flow, Area 1 


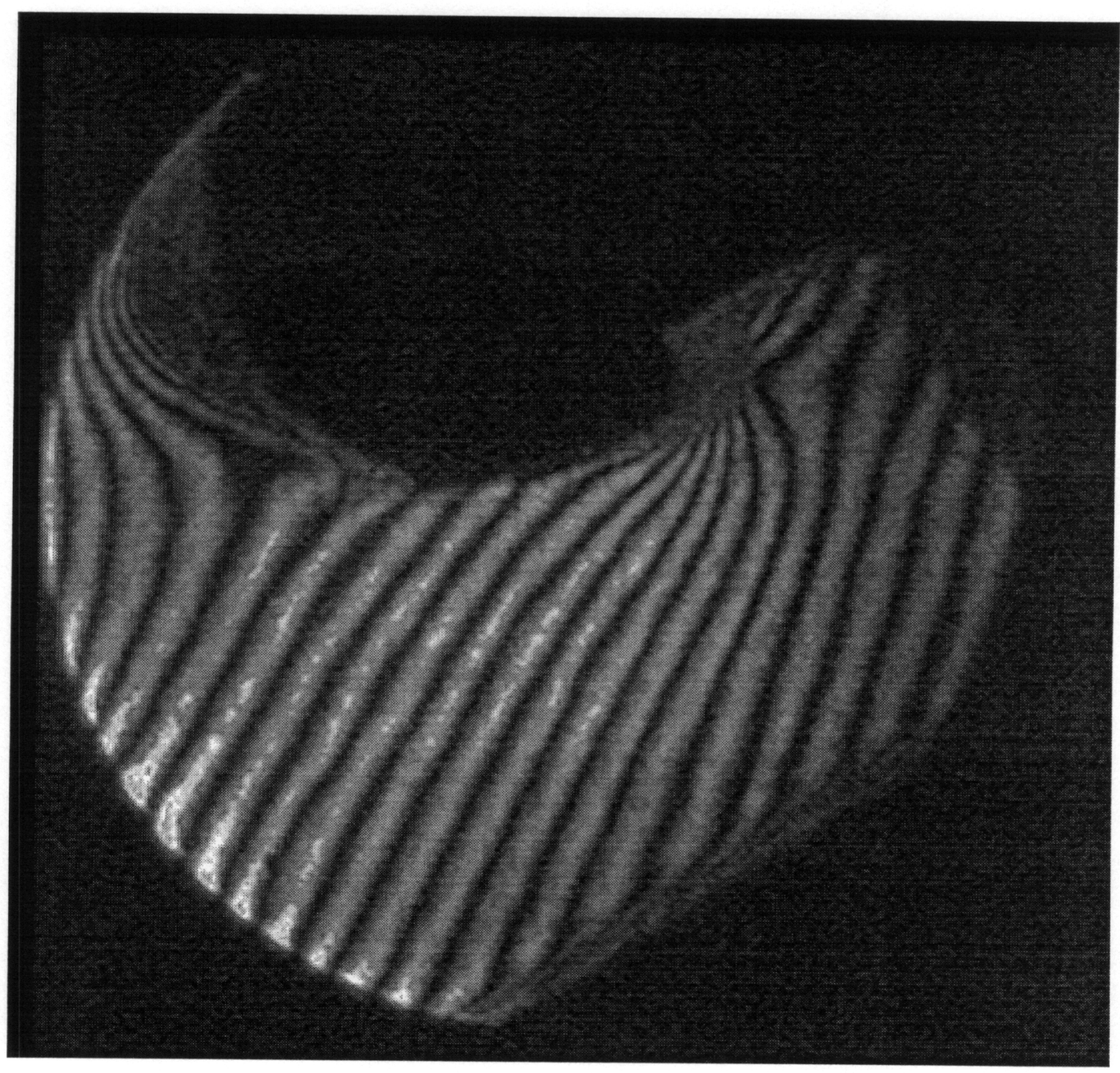

Figure 6.2: Interferogram of Unheated Flow, Area 2 


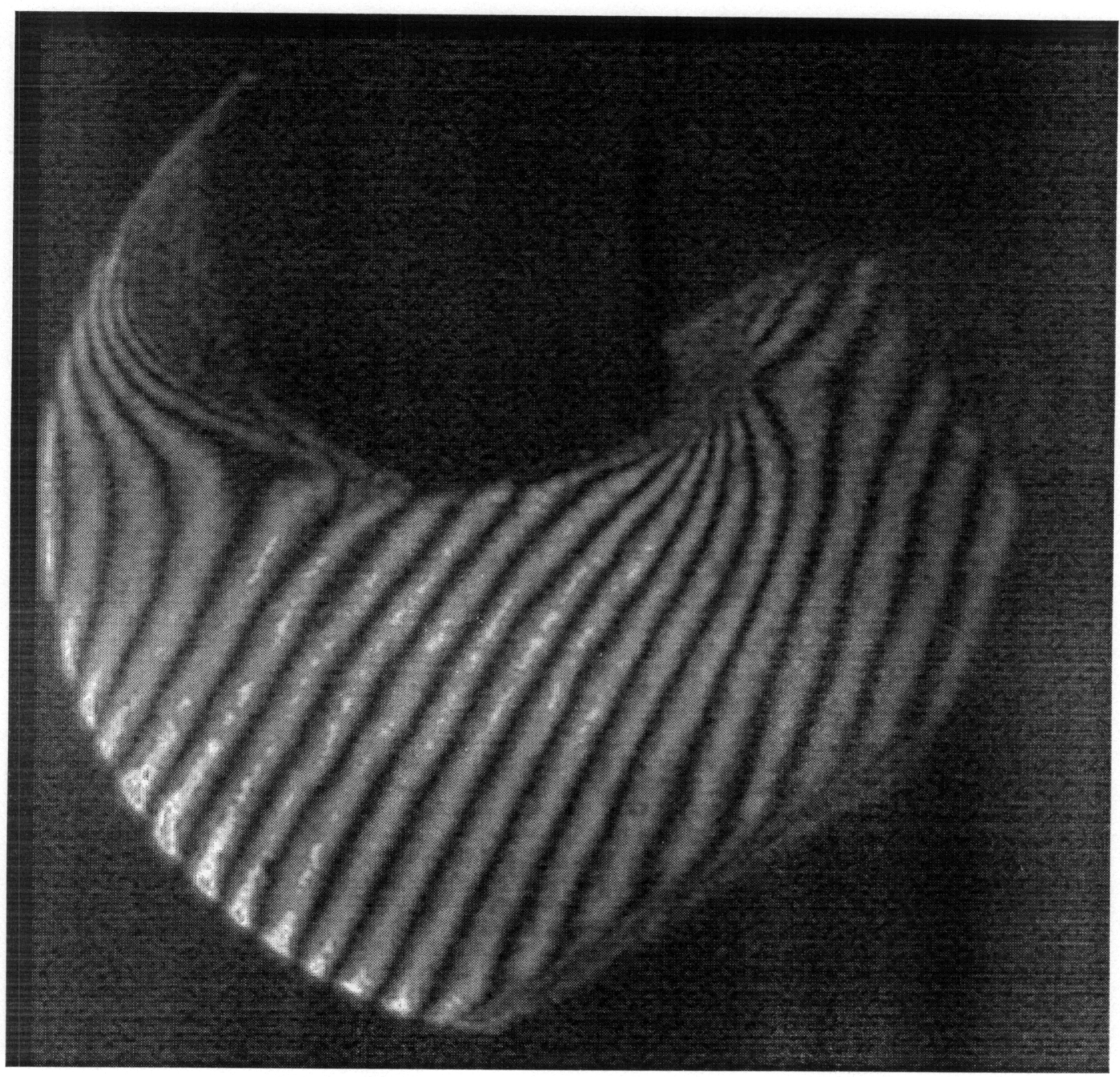

Figure 6.2: Interferogram of Unheated Flow, Area 2 


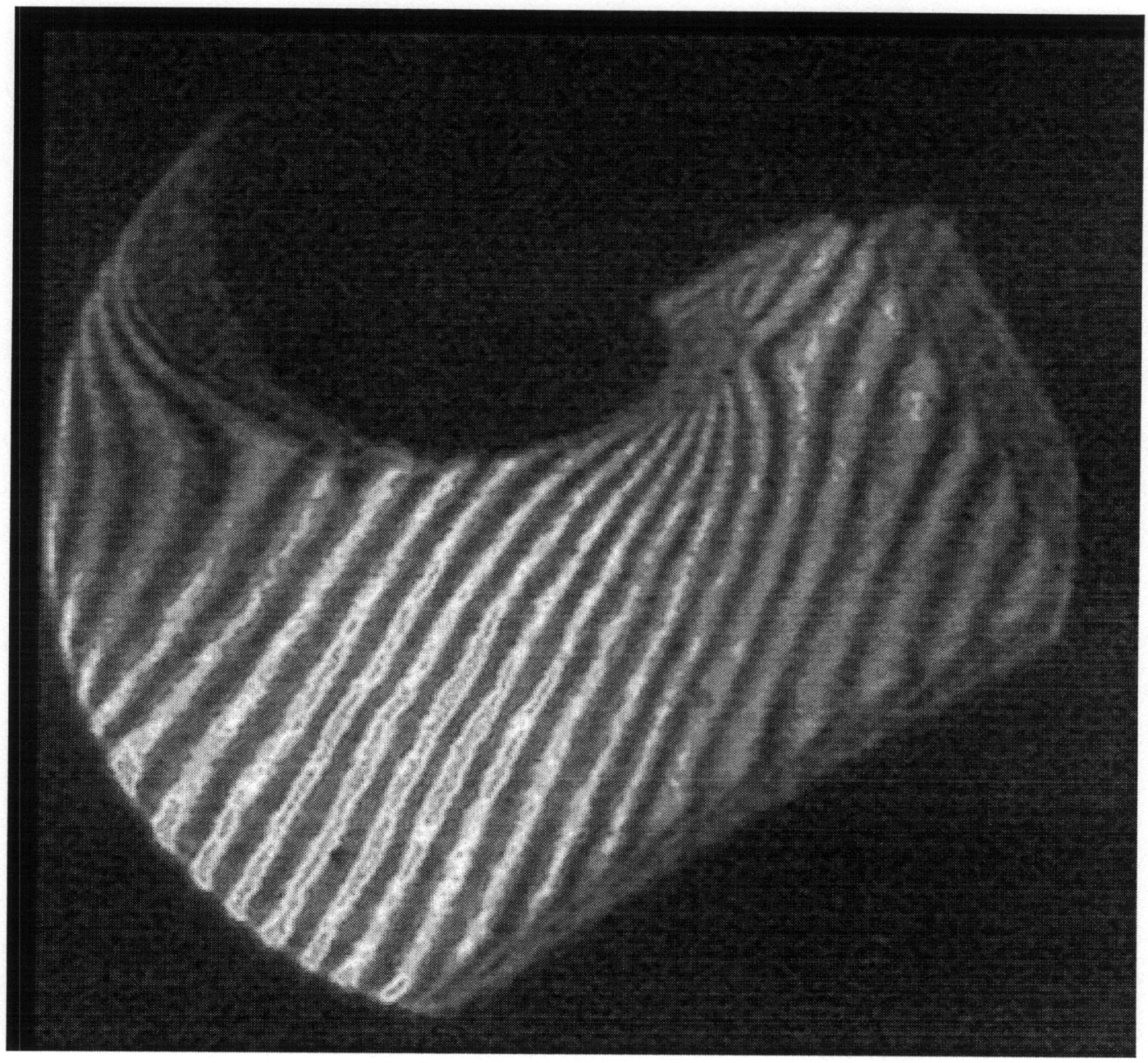

Figure 6.3: Interferogram of Heated Flow, Area 1 


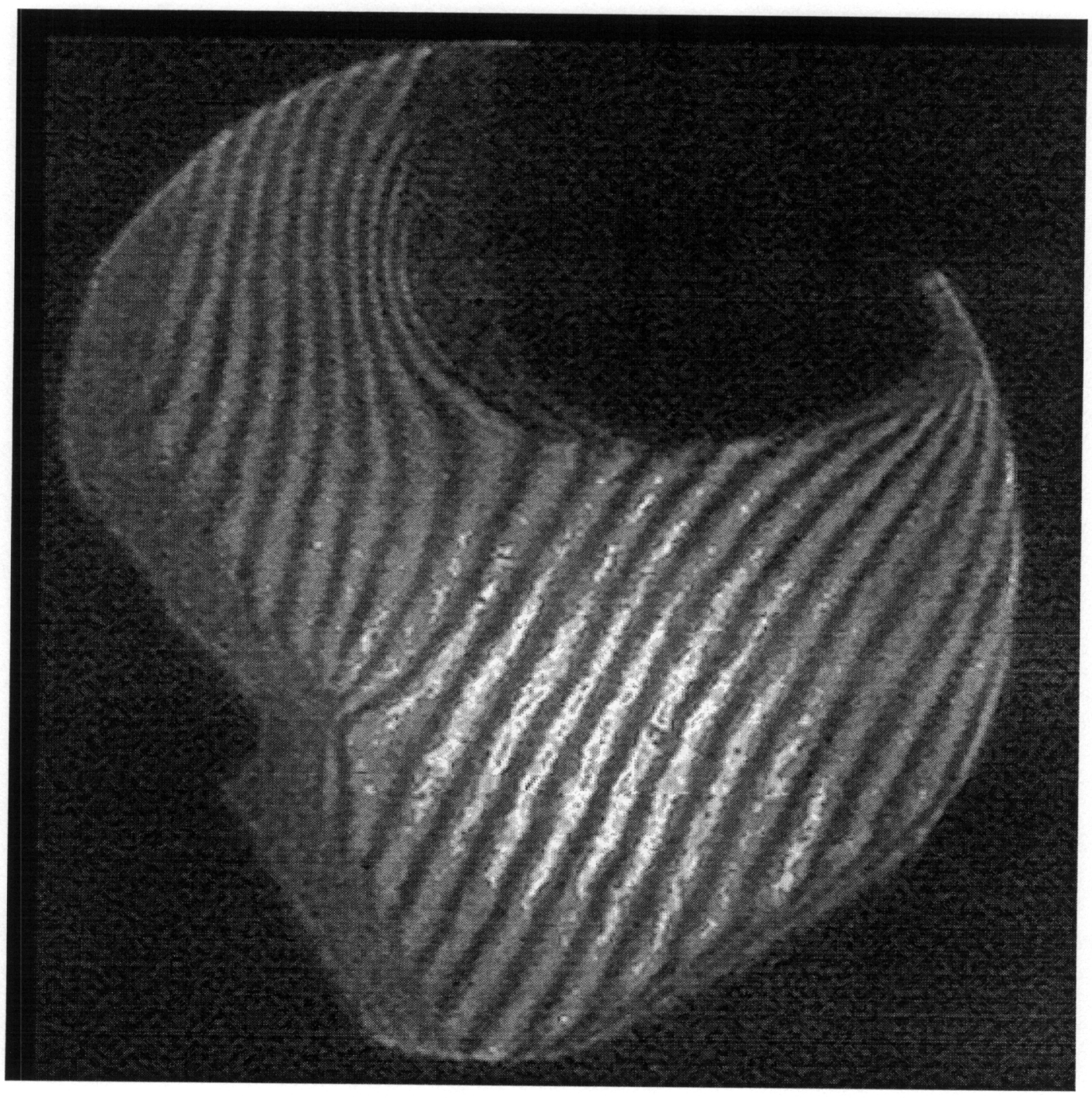

Figure 6.4: Interferogram of Heated Flow, Area 2 


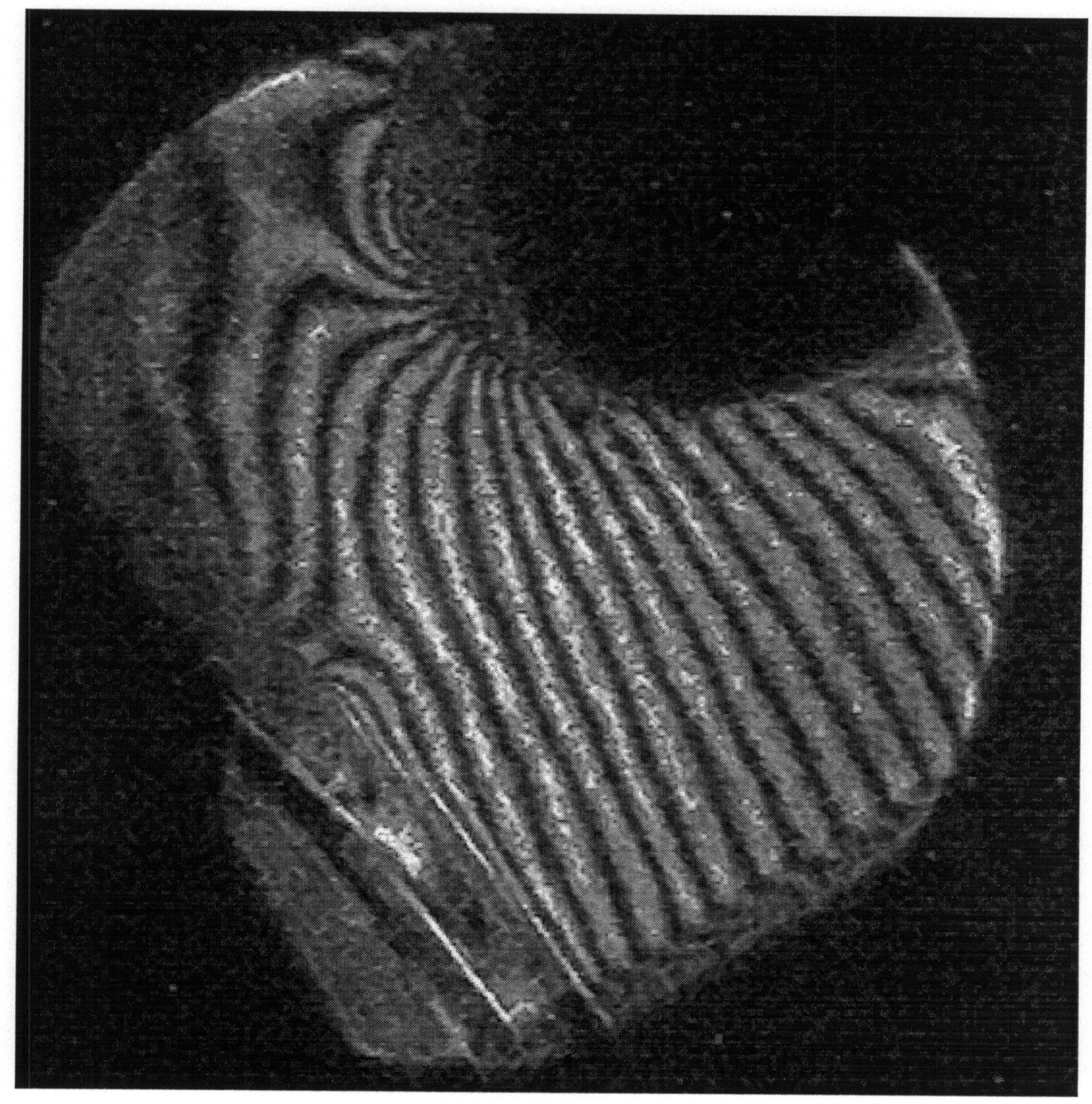

Figure 6.5: Unsteady Interferogram in Area 1, $178 \mu s$ Delay 


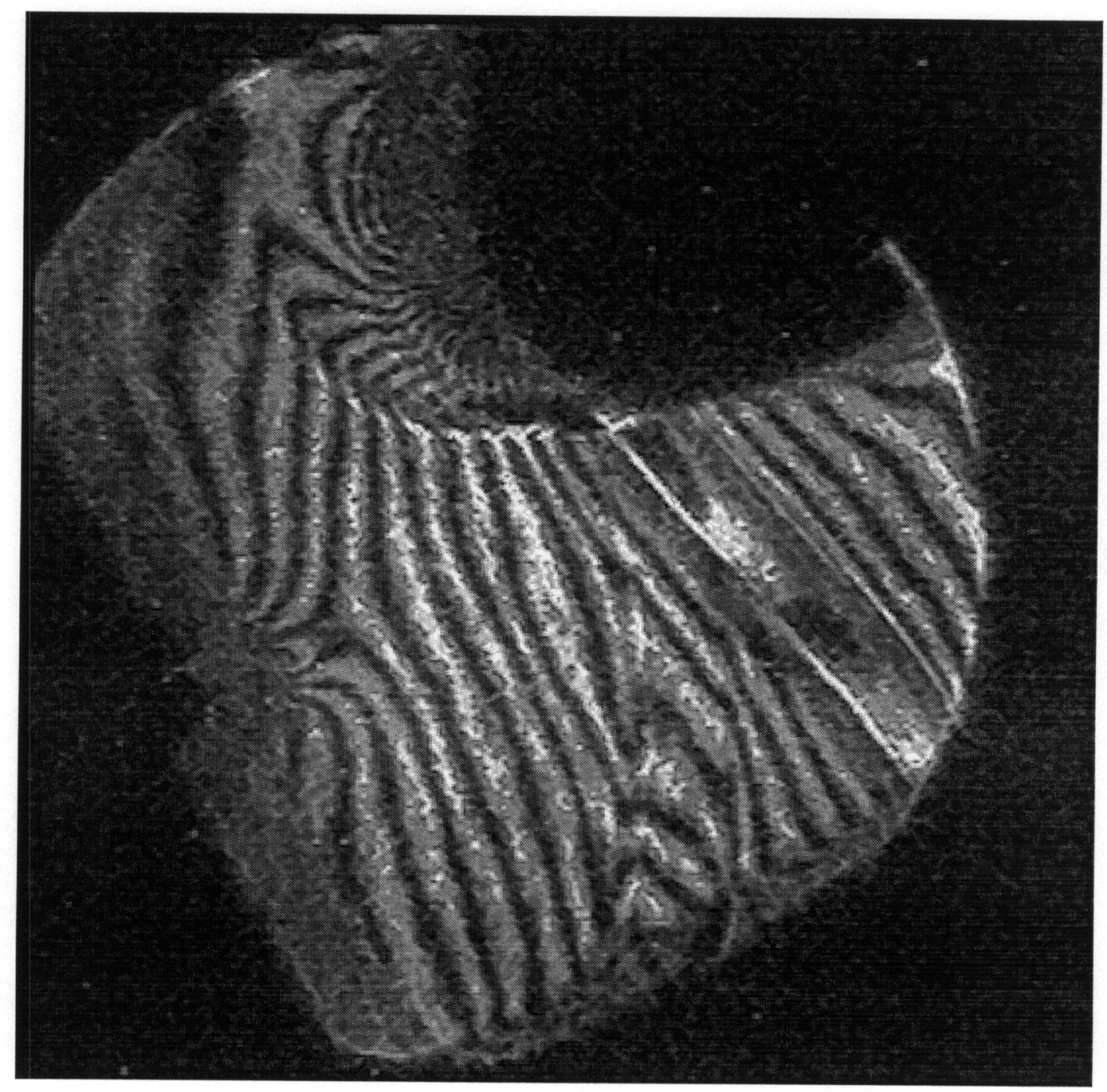

Figure 6.6: Unsteady Interferogram in Area 1, $258 \mu s$ Delay 


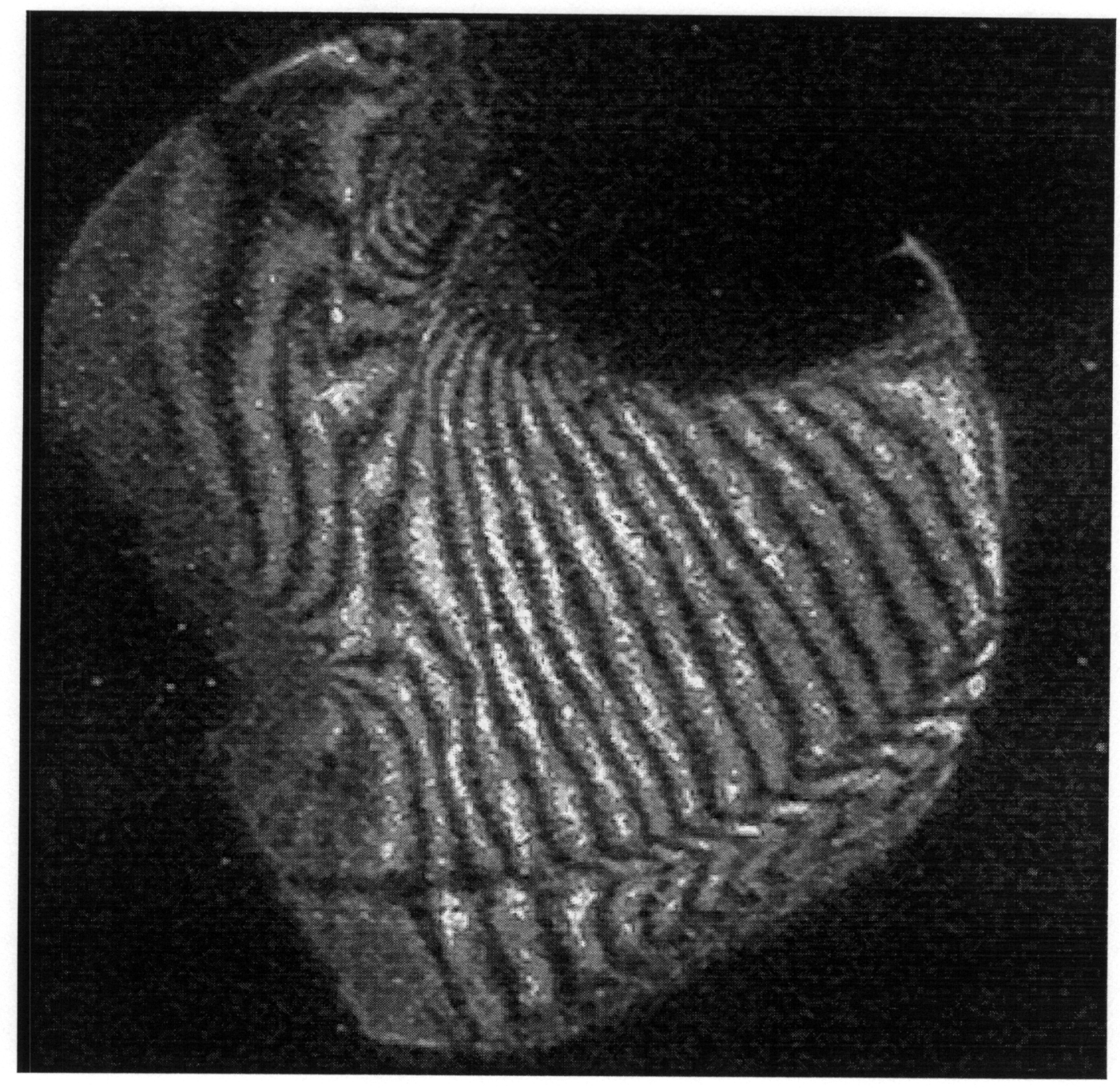

Figure 6.7: Unsteady Interferogram in Area 1, $337 \mu s$ Delay 


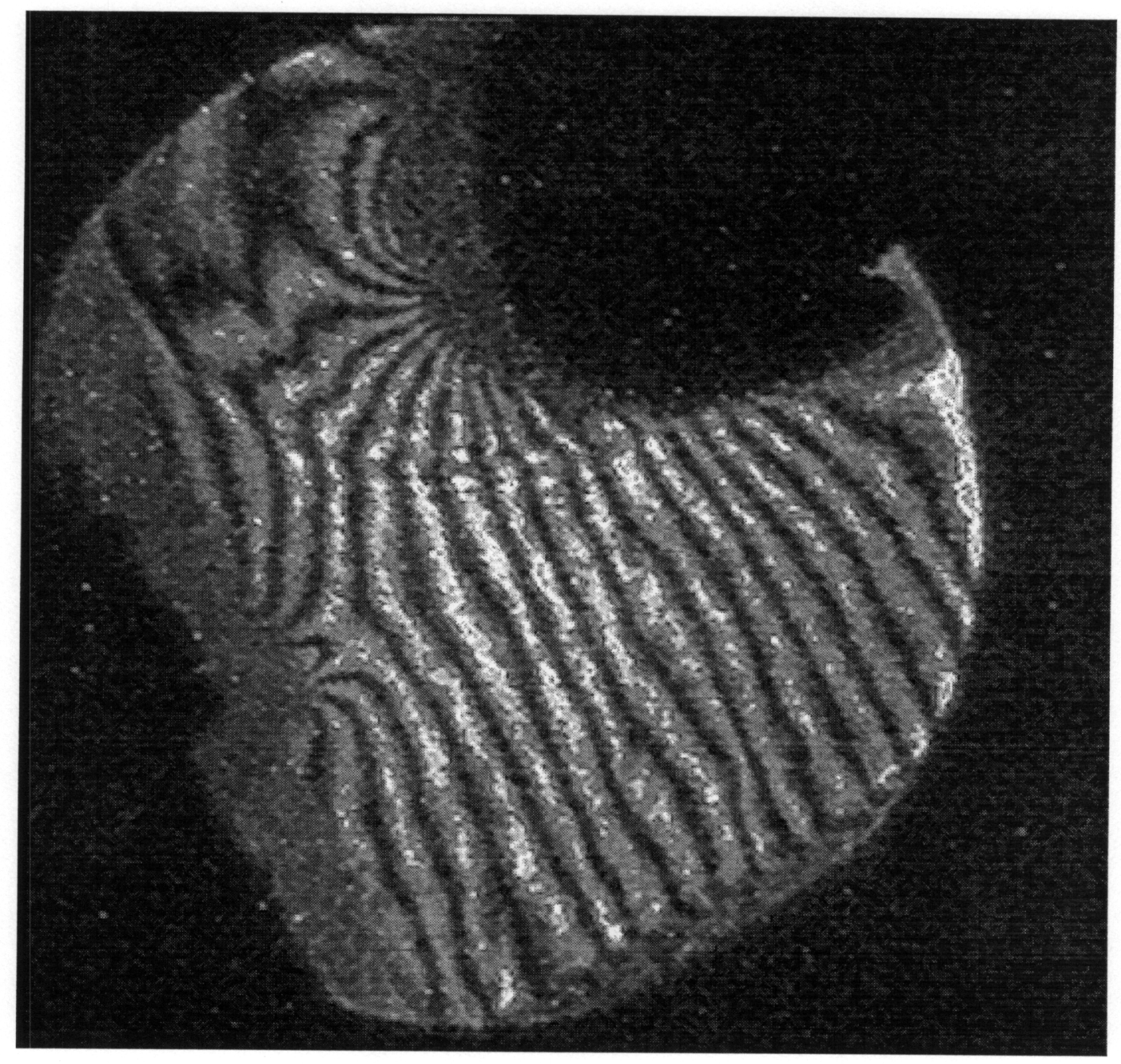

Figure 6.8: Unsteady Interferogram in Area 1, $393 \mu s$ Delay 


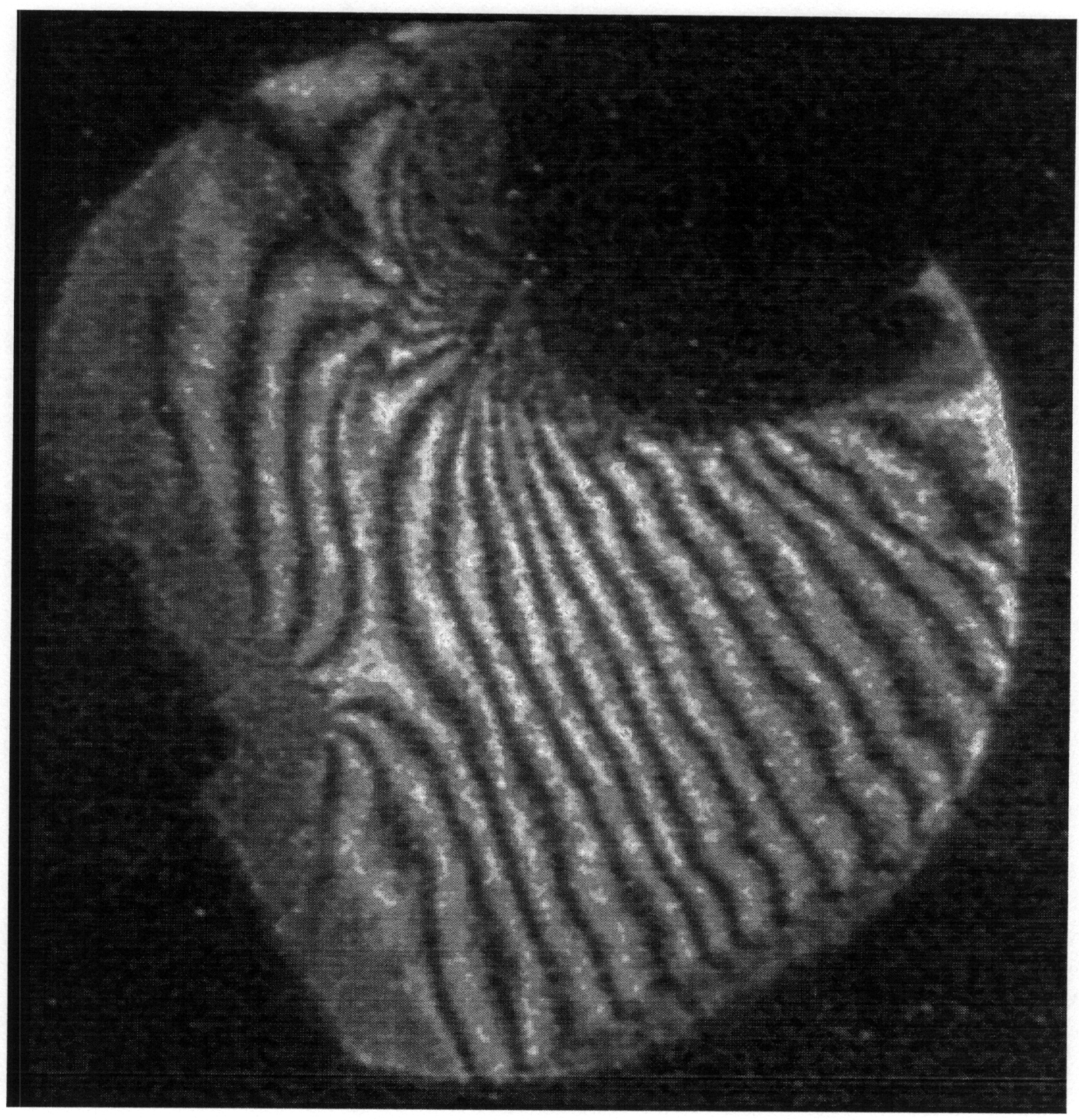

Figure 6.9: Unsteady Interferogram in Area 1, $465 \mu s$ Delay 


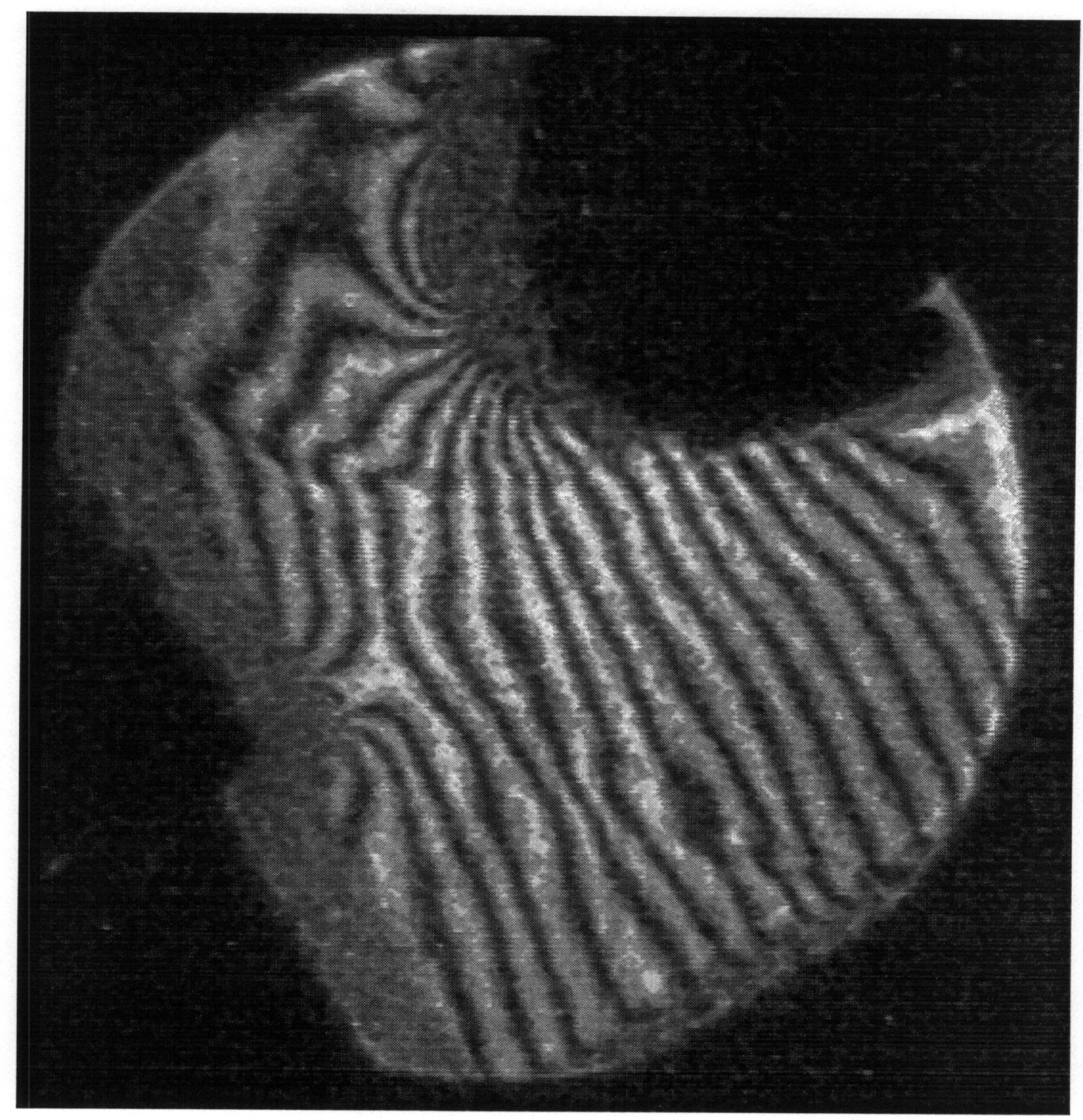

Figure 6.10: Unsteady Interferogram in Area 1, $518 \mu s$ Delay 


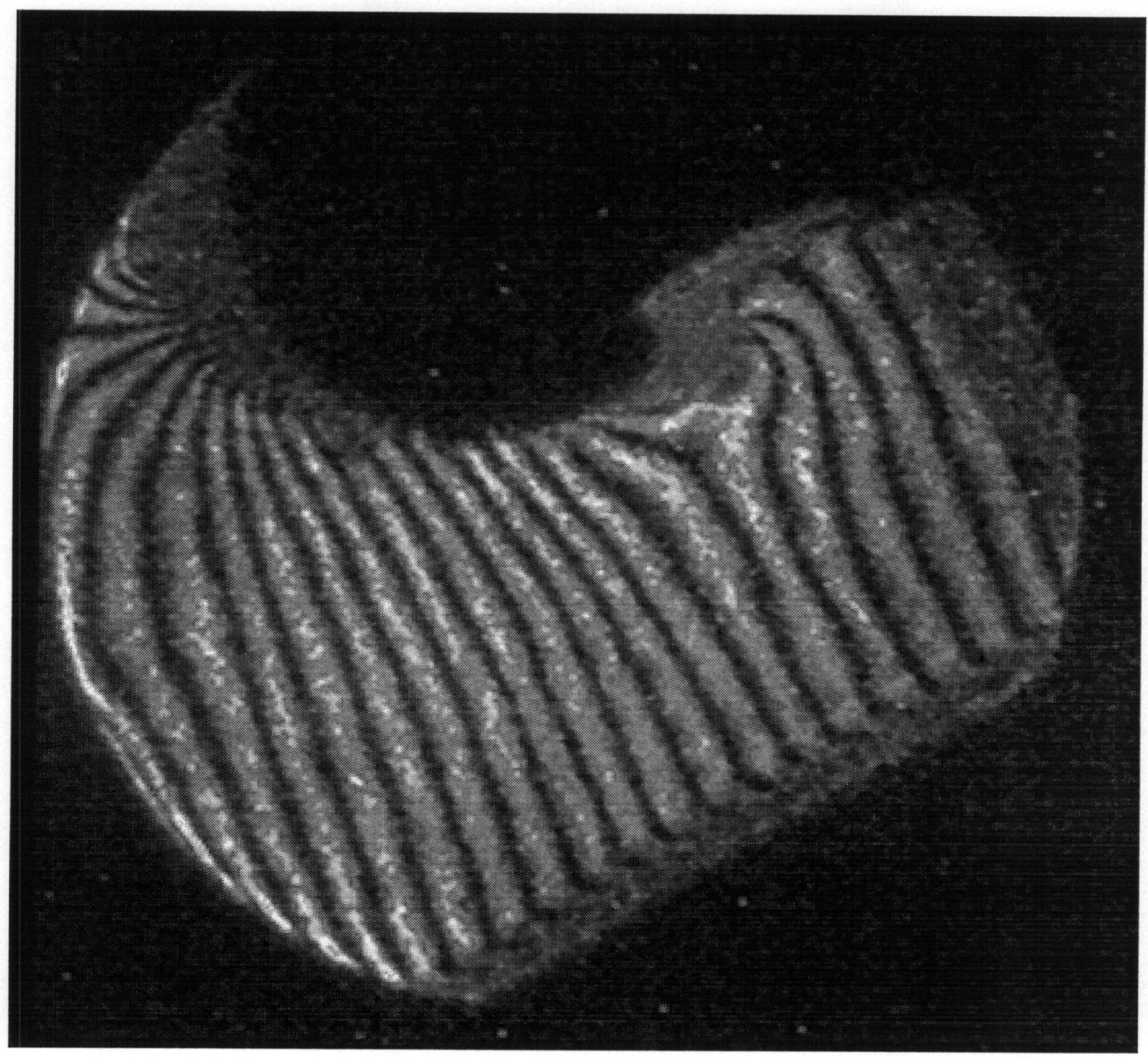

Figure 6.11: Unsteady Interferogram in Area 2, $178 \mu s$ Delay 


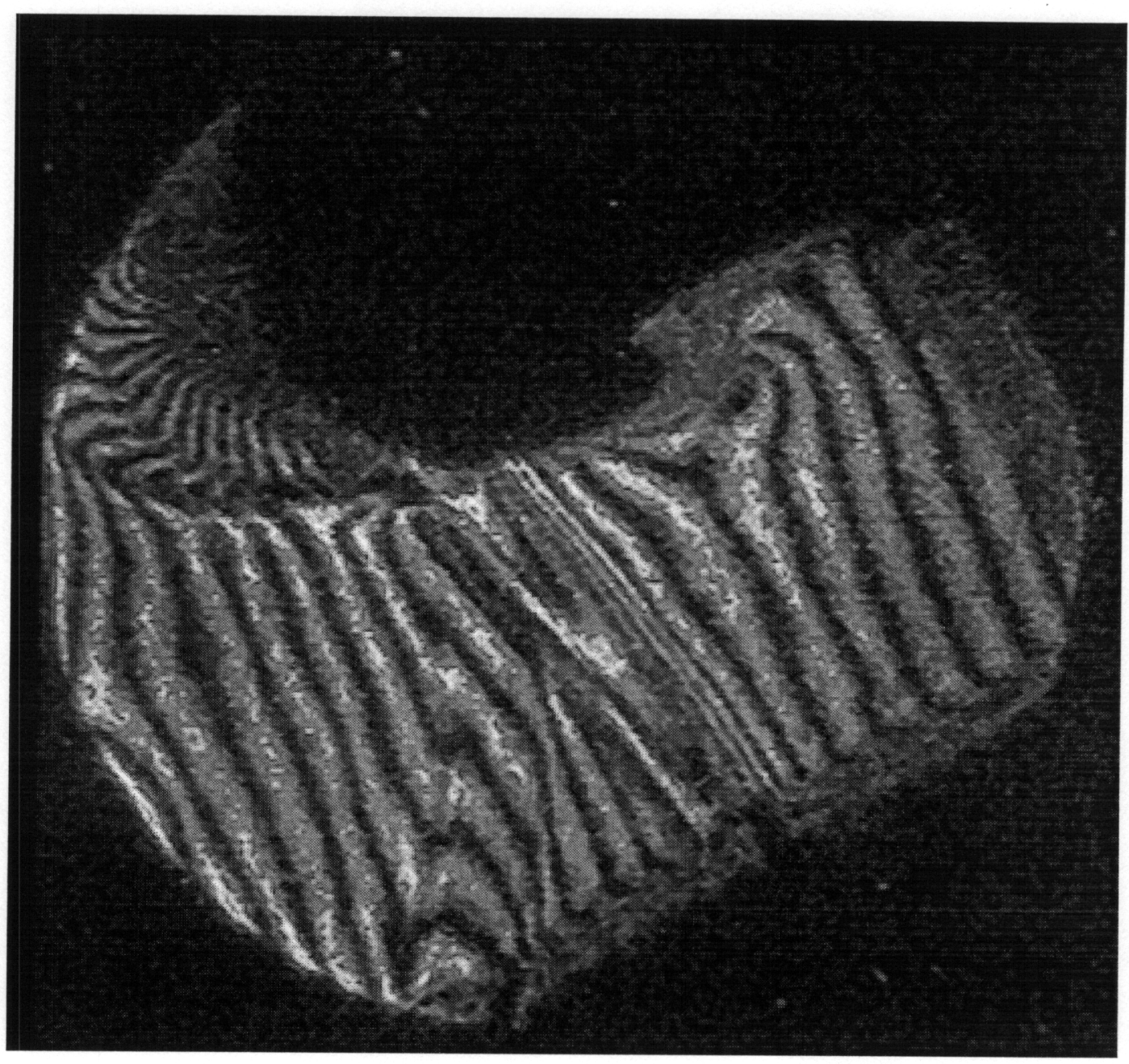

Figure 6.12: Unsteady Interferogram in Area 2, $258 \mu s$ Delay 


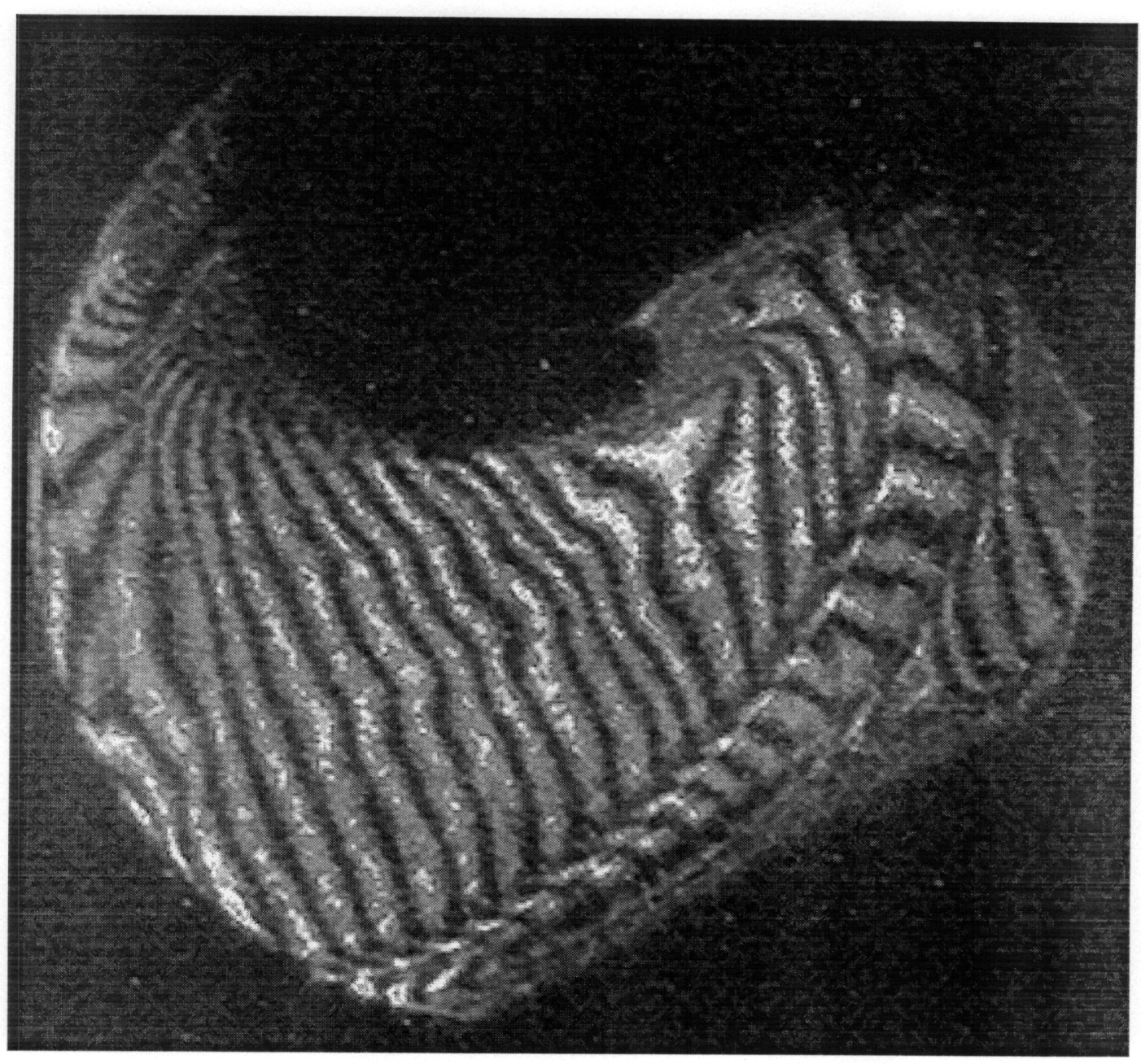

Figure 6.13: Unsteady Interferogram in Area 2, $337 \mu s$ Delay 


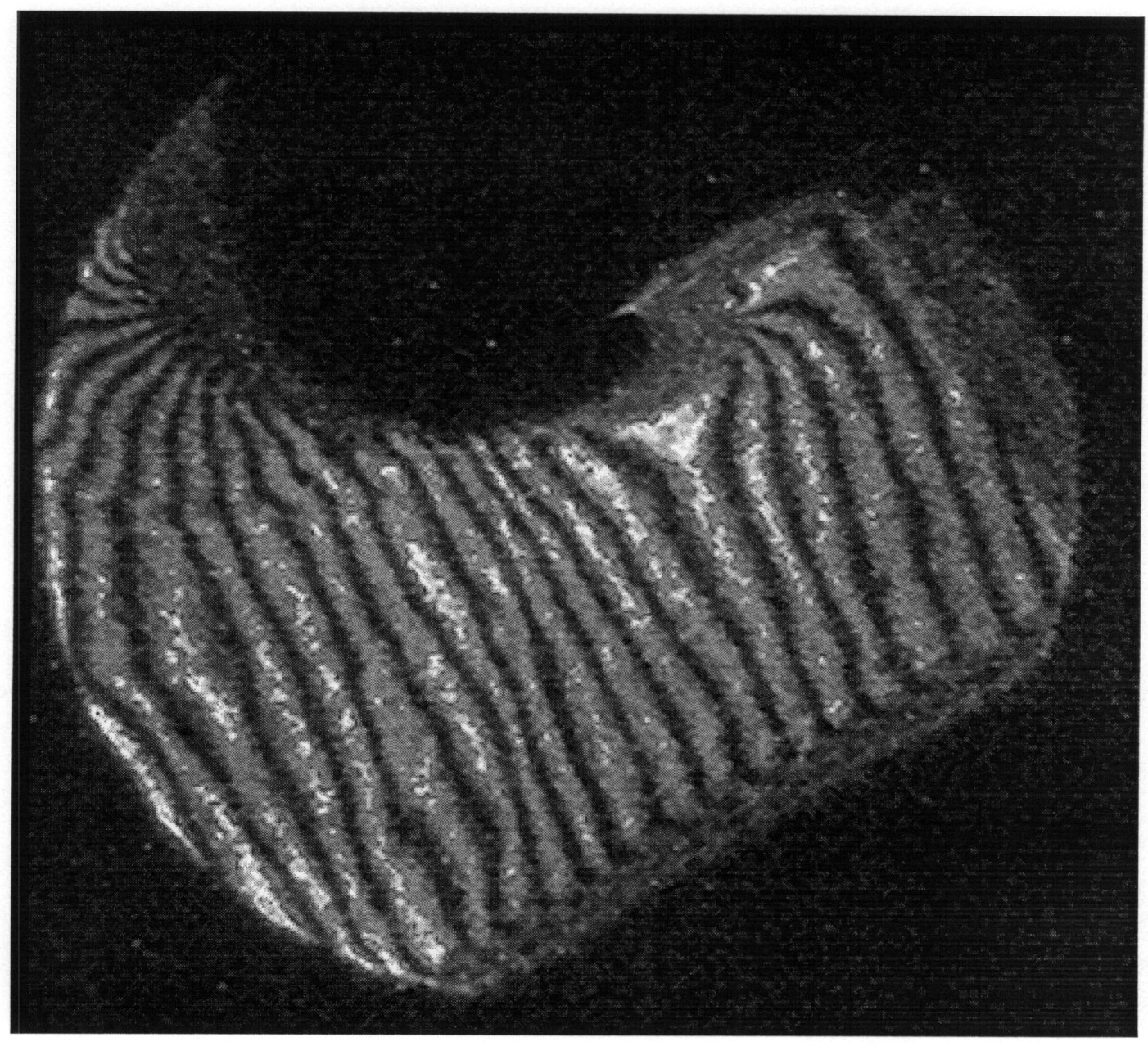

Figure 6.14: Unsteady Interferogram in Area 2, $393 \mu s$ Delay 


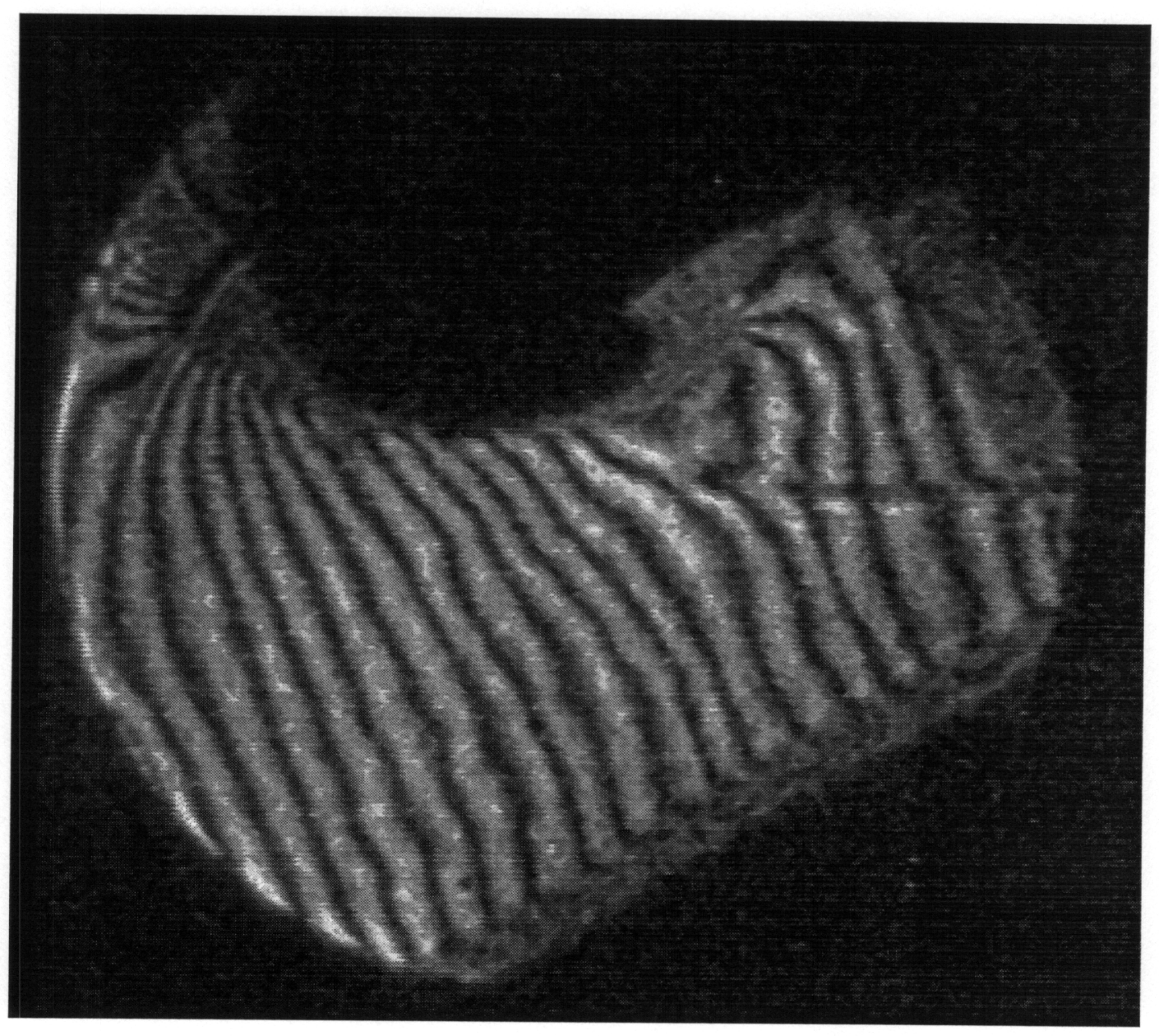

Figure 6.15: Unsteady Interferogram in Area 2, $465 \mu s$ Delay 


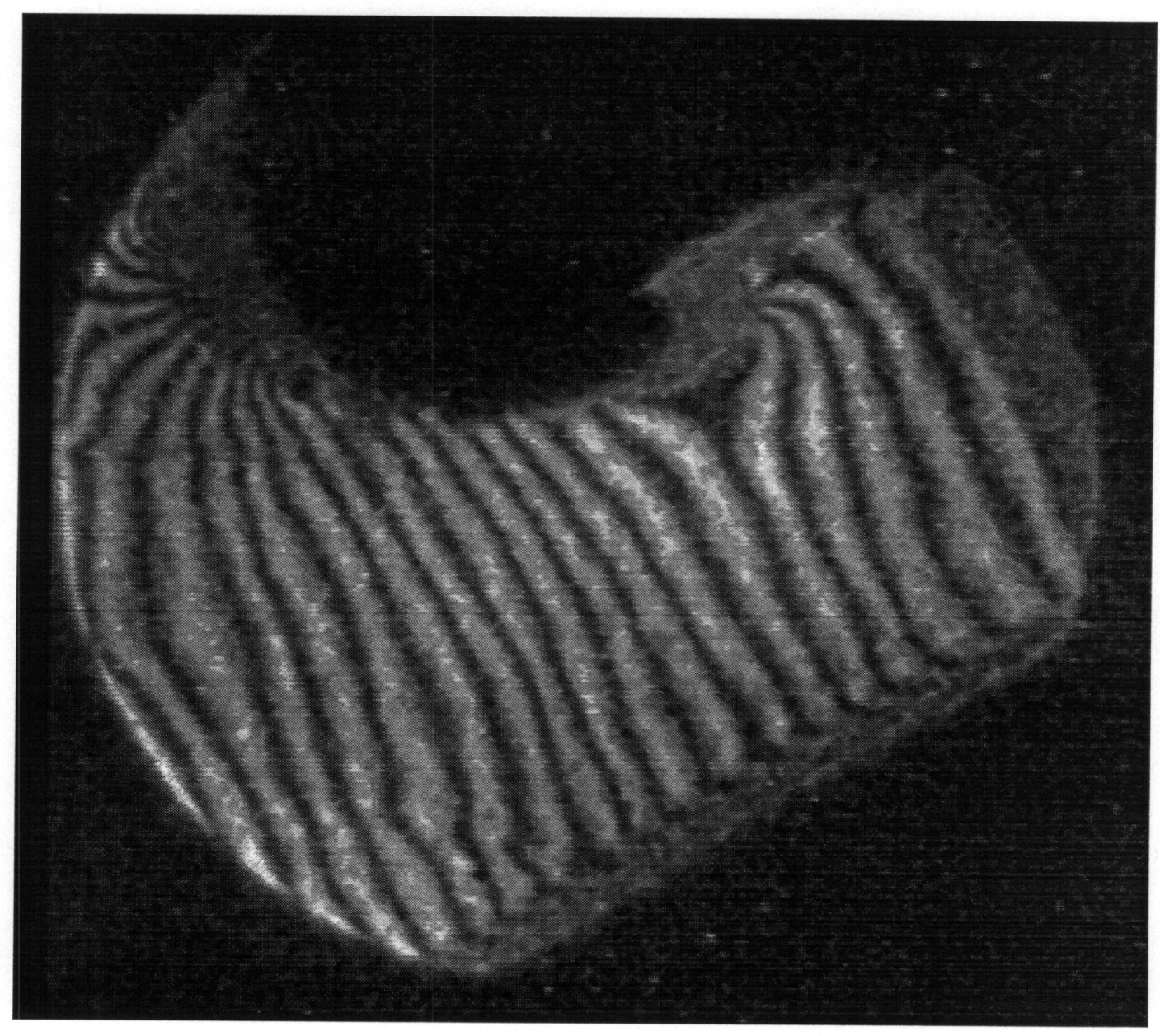

Figure 6.16: Unsteady Interferogram in Area 2, $518 \mu s$ Delay 


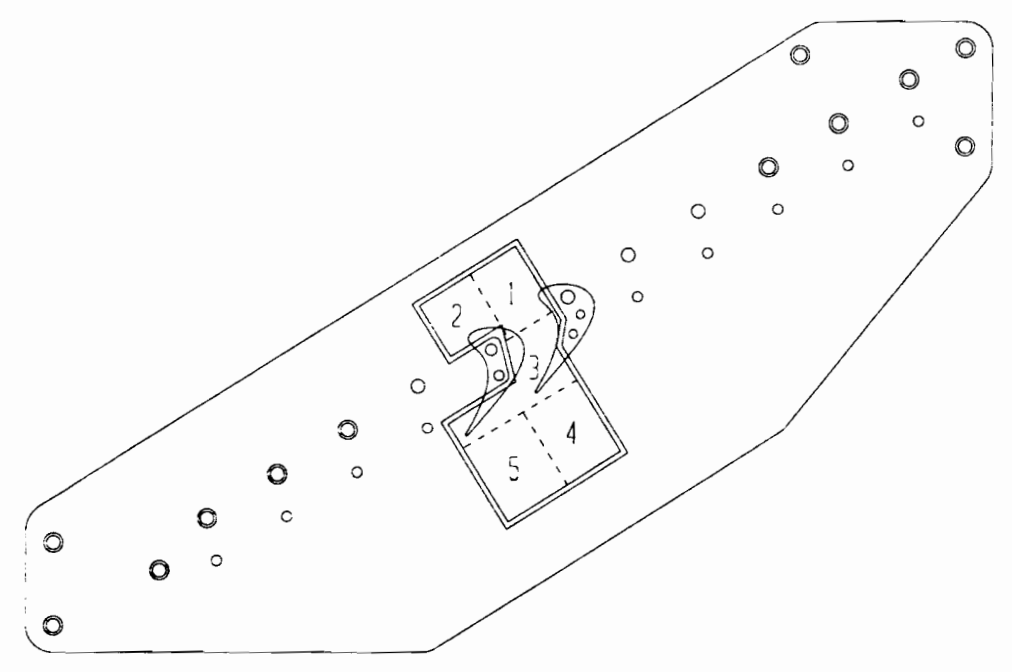

Figure 6.17: Relative Positioning of Area 1 and Area 2 to the Studied Blade Passage 
Figure 6.18 shows the density field results for the steady, adiabatic case. Portions of the blade are also shown for reference. As expected, the flow away from the cascade, toward the inlet is fairly uniform with a density value ranging from between 2.4 and $2.5 \frac{\mathrm{kg}}{\mathrm{m}^{3}}$. Also, the decrease in density as the flow enters the blade passage corresponds to the increase in velocity experienced by the flow as it is accelerated toward sonic velocity at the throat. Another feature of note is the increased density values near the LE of the blade in the left hand lower corner of the plot where the flow is slowed by the presence of the blade. The extremely dark region in the lower corner is where the edge of the CCD image was encountered.

The steady, heated density field is presented in Fig. 6.19. The general flow features match those found for the steady, adiabatic case, with an increase in density near the blade leading edge and a decrease in density as the flow enters the blade passage. Also as expected, due to the heating of the flow, the general magnitude of the inlet density has decreased to between 2.0 to $2.1 \frac{\mathrm{kg}}{\mathrm{m}^{3}}$. This is a decrease of about $12.5 \%$.

Now the unsteady results will be presented. The first density field is given at a delay time of $178 \mu \mathrm{s}$. This time was chosen because the initial shock is just entering the studied domain, therefore the flowfield is generally undisturbed. It should be noted, however, that even though a shock is normally associated with supersonic events, the passing shock is propagating into a subsonic flow, therefore it can influence the upstream flowfield. Again, the same general flow features as found in the steady cases are found, though less pronounced. This could be attributed to two factors. First, the presence of the shock shaper and the passing shock wave could be modifying the flowfield slightly. Second, since the steady interferograms were taken in the winter and the unsteady interferograms were taken in the summer, the slight increase in the ambient temperature could have slightly modified the flowfield.

In Fig. 6.21, taken at a delay time of $258 \mu \mathrm{s}$, the presence of the shock is clearly seen. The density behind the shock has increased to over $2.7 \frac{\mathrm{kg}}{\mathrm{m}^{3}}$, and the acceleration of the flow into the passage has decreased in upstream extent. The drop in density ahead of the shock was not expected. Some speculation on the cause of this phenomena is perhaps the presence of the shock wave is producing 'blockage' in the cascade. The upstream flow velocity in the tunnel is set by the mass flow rate, if 


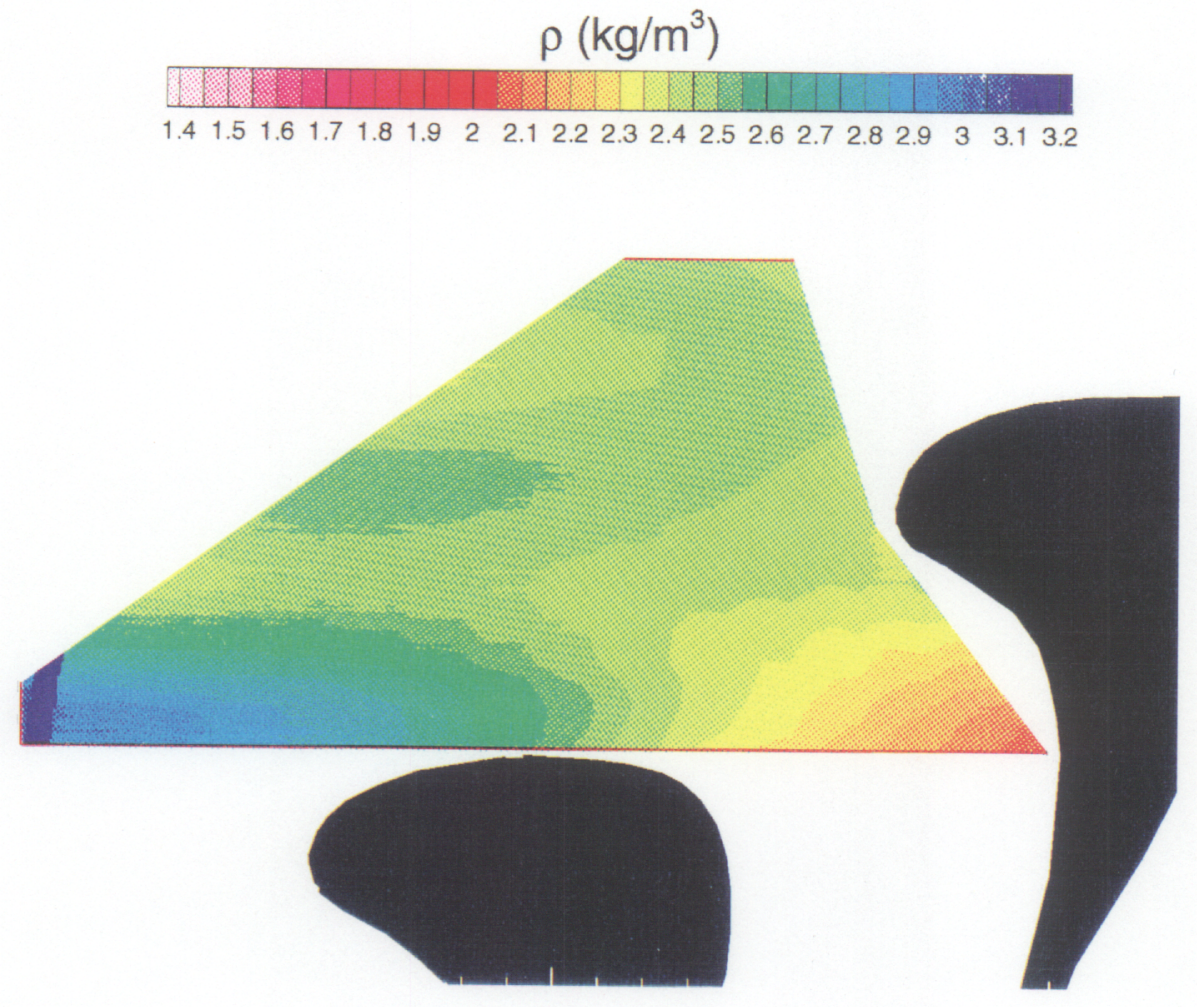

Figure 6.18: Density Field for Adiabatic Flow 

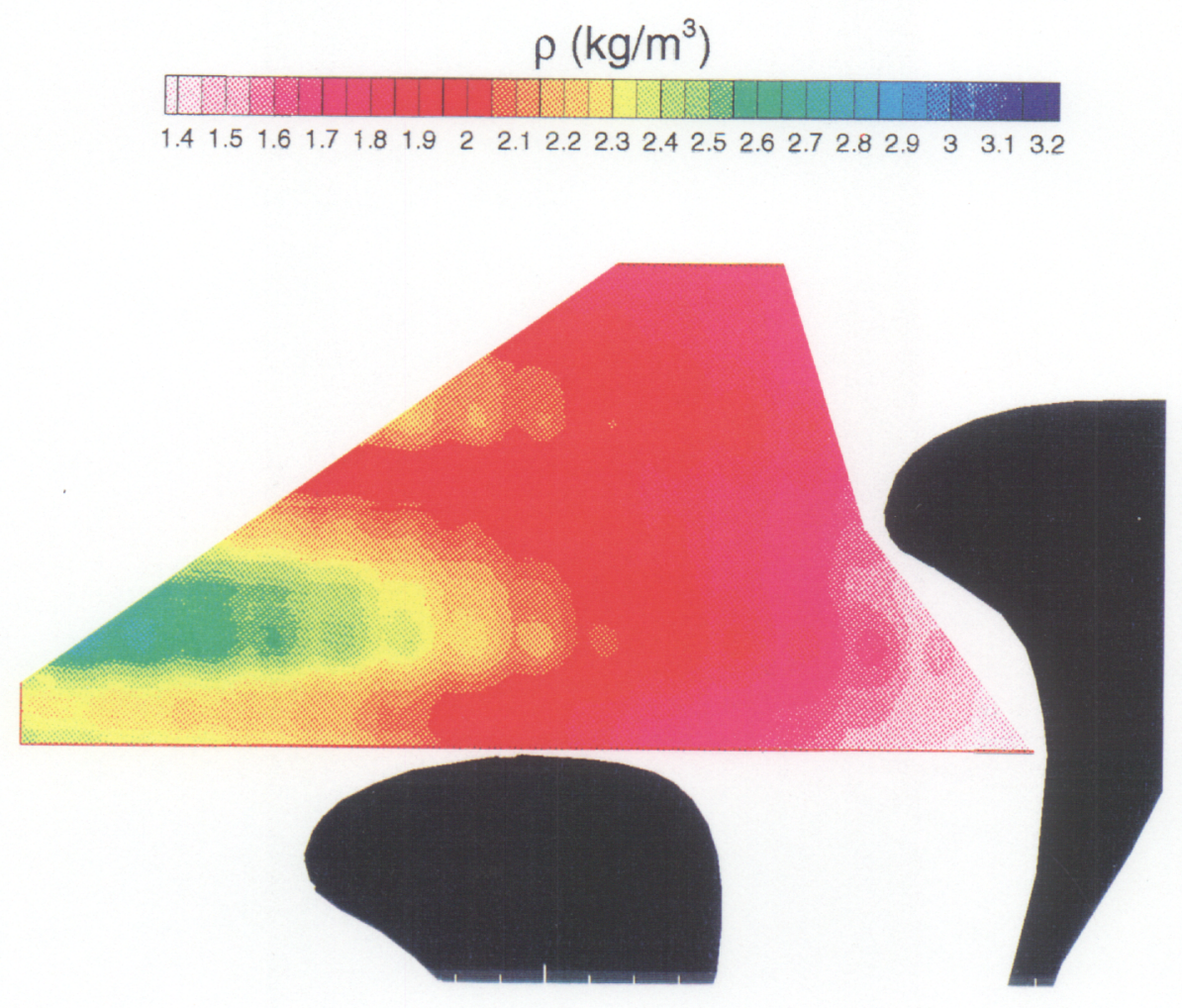

Figure 6.19: Density Field for Heated Flow 
$\rho\left(\mathrm{kg} / \mathrm{m}^{3}\right)$

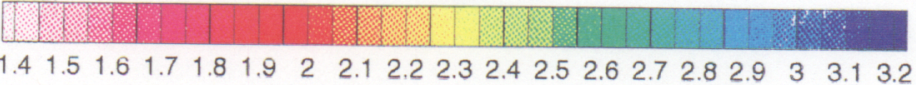

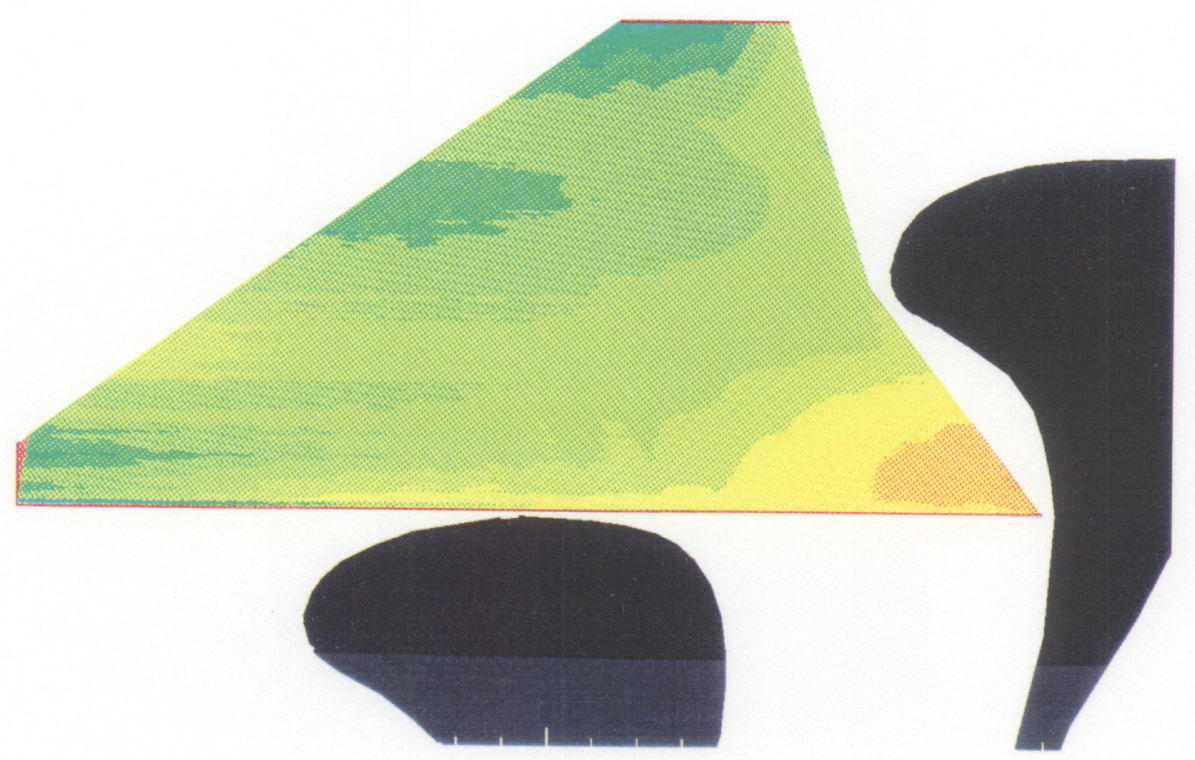

Figure 6.20: Density Field for Unsteady Flow, $178 \mu s$ Delay 
the presence of the shock is seen as reducing the available flow area, the flow could accelerate ahead of the shock passing, causing the observed decrease in density.

As the shock moves further upstream, the reflection generated as it passed over the studied blade propagates upstream and is moving out of the studied area by the time the next interferogram, Fig. 6.22, taken at a delay time of $337 \mu \mathrm{s}$. Again, the presence of the shock wave is clearly visible in the density field. The density around the suction surface of the lower blade is low around most of the blade. The expected decrease in density in the blade passage, near the pressure surface of the higher blade in no longer present.

At a delay time of $393 \mu s$, the flow has again begun to accelerate into the blade passage along the pressure surface of the upper blade, Fig. 6.23. The flow also shows an increase in density around the LE of the lower blade. The upstream density level has remained elevated near a value of 2.6 to $2.7 \frac{\mathrm{kg}}{\mathrm{m}^{3}}$.

By a delay time of $465 \mu \mathrm{s}$, another strong reflection has entered the studied area as seen in Fig. 6.24. The reflection is apparent in the lower, left-hand corner of the studied area. The flow has again accelerated behind the shock. Also, the flowfield density is, in fact, increasing along the pressure surface of the upper blade.

By a delay time of $518 \mu s$, any reflected shock waves should be extremely weak. The overall density level upstream of the cascade remains elevated; see Fig. 6.25. However, it is expected that if interferograms were taken at much longer delay times, the elevated levels would drop down near the undisturbed value. The flowfield appears to be taking on the general features associated with the undisturbed flow, with a slight flow acceleration into the blade passage and slightly changed density values far upstream of the cascade. 

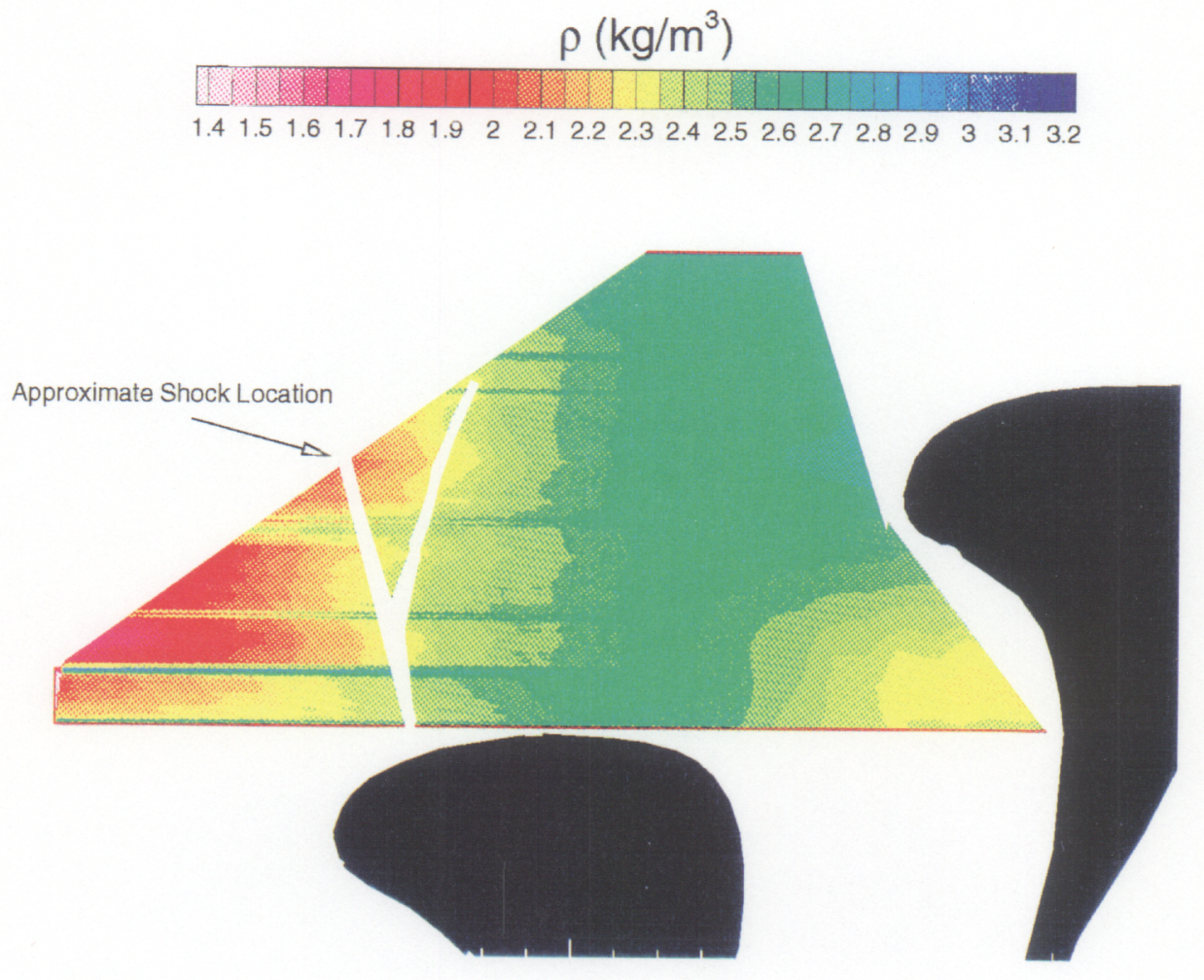

Figure 6.21: Density Field for Unsteady Flow, $258 \mu s$ Delay 

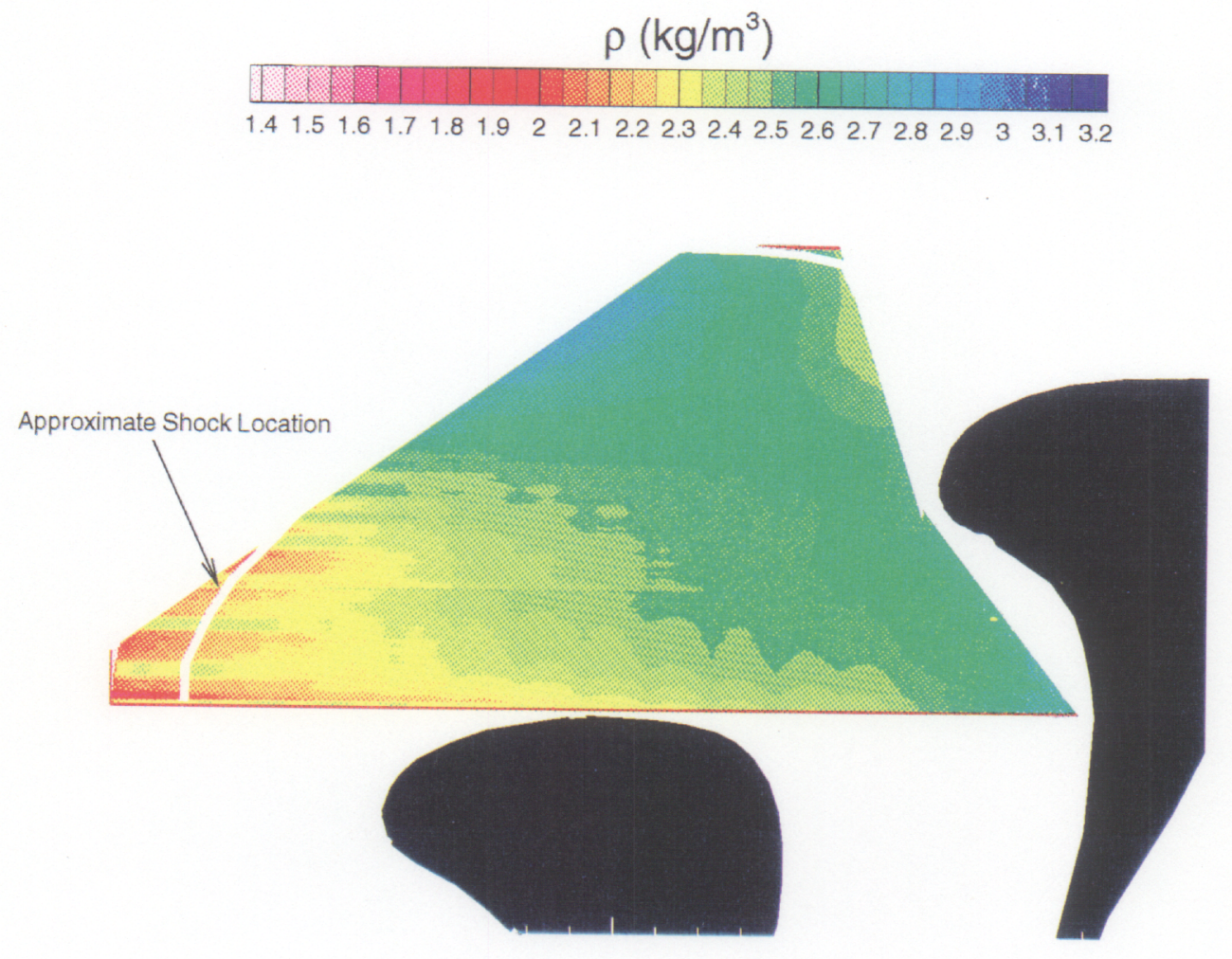

Figure 6.22: Density Field for Unsteady Flow, $337 \mu s$ Delay 

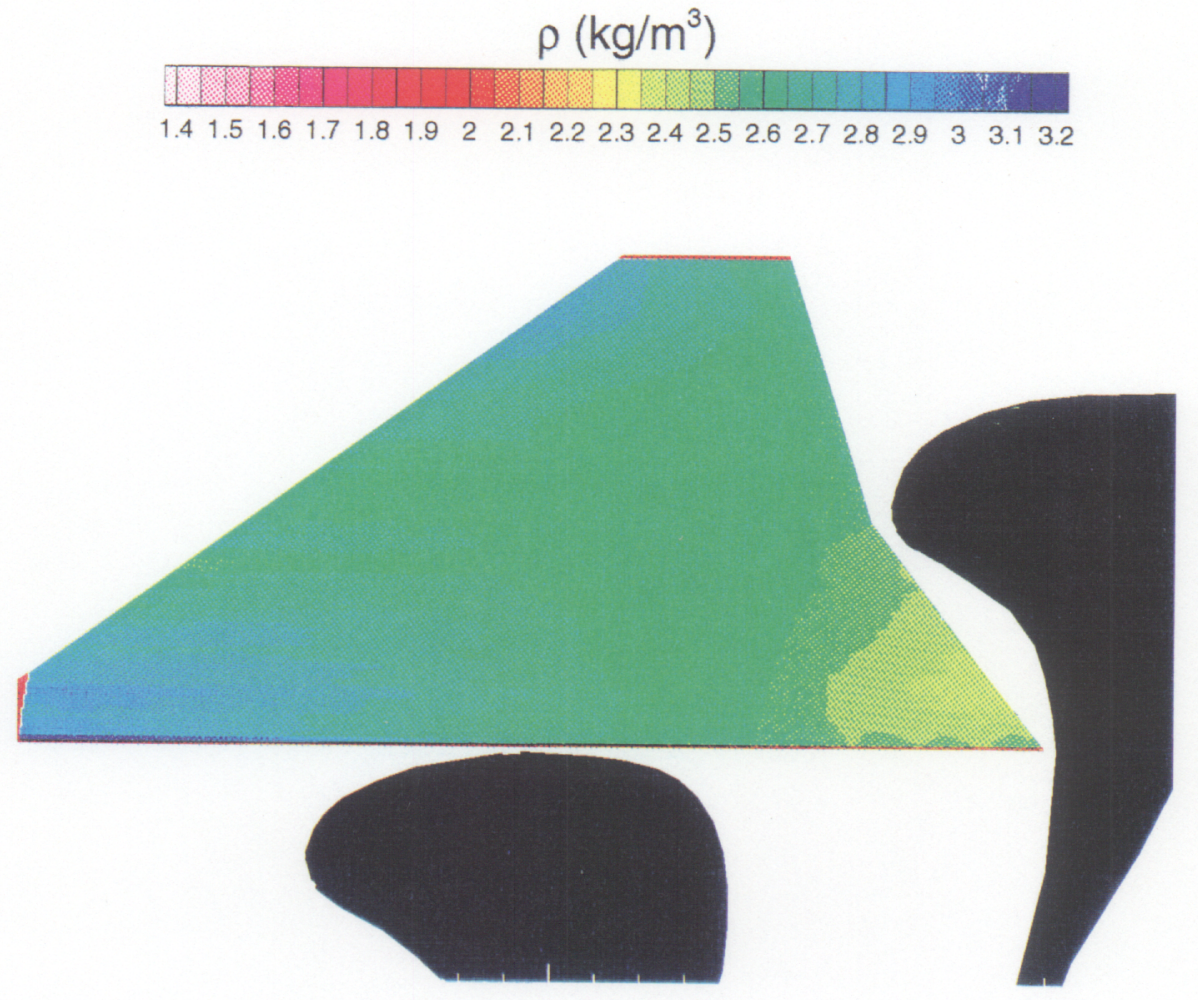

Figure 6.23: Density Field for Unsteady Flow, $393 \mu s$ Delay 

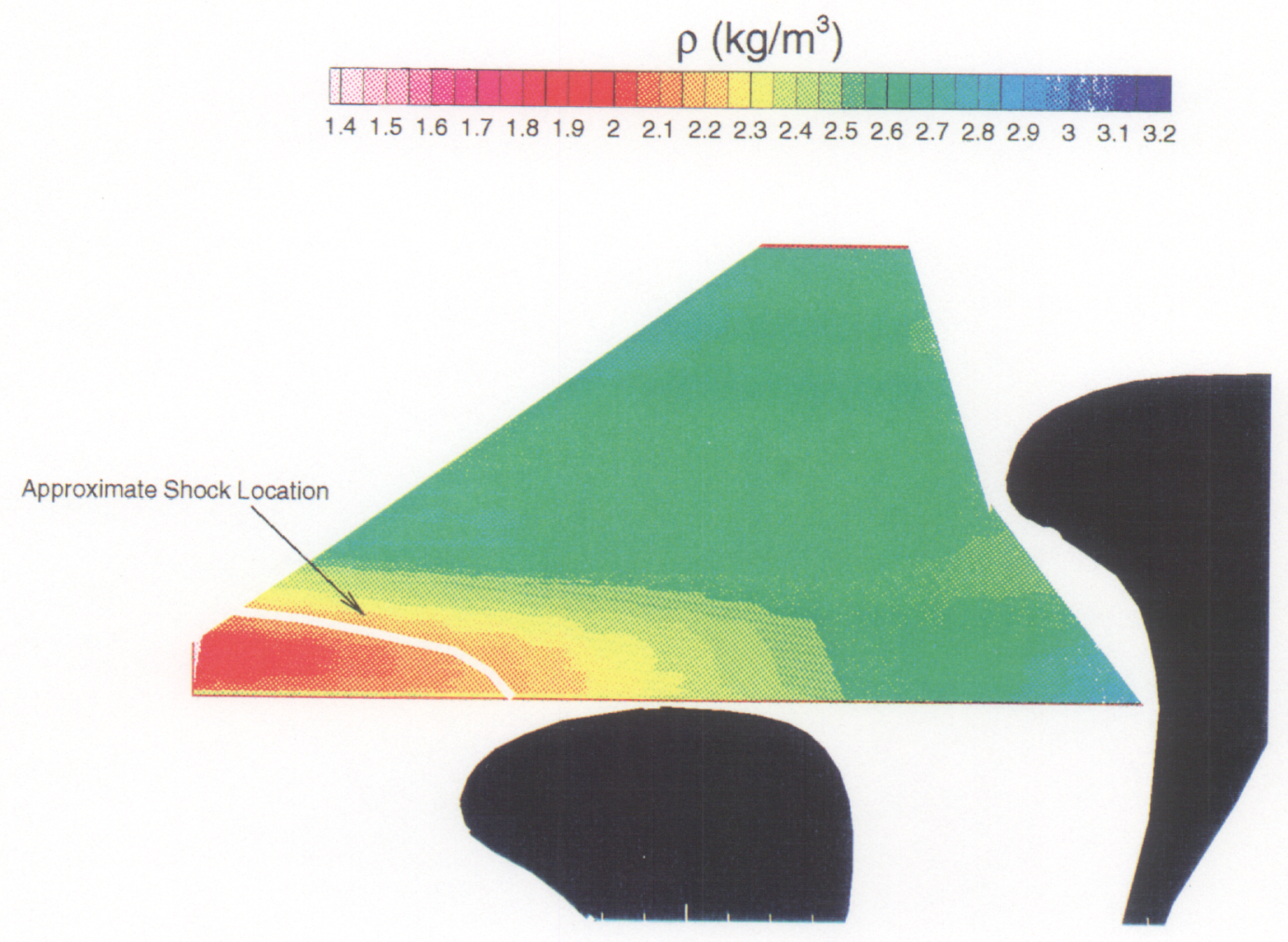

Figure 6.24: Density Field for Unsteady Flow, $465 \mu s$ Delay 

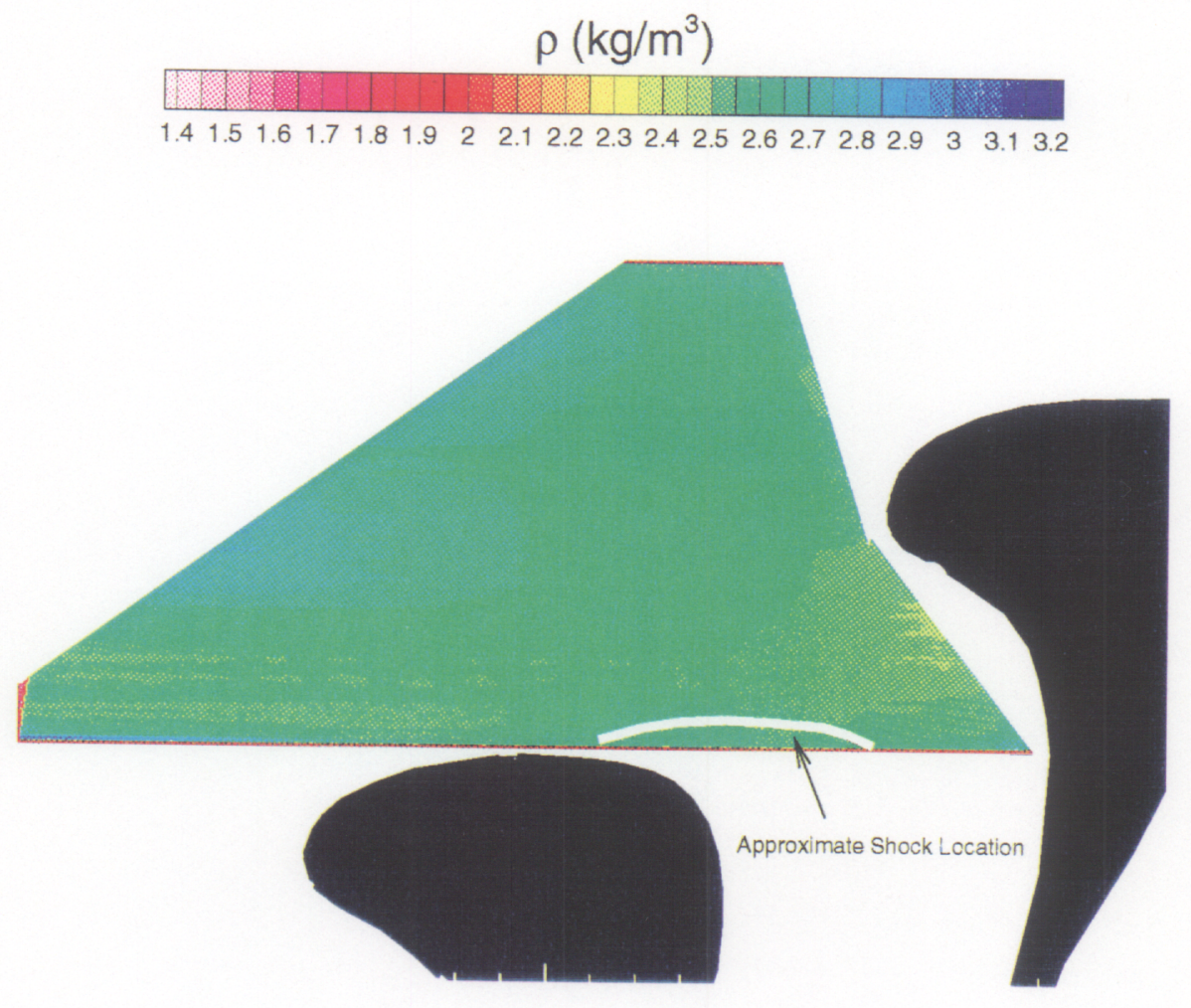

Figure 6.25: Density Field for Uusteady Flow, $518 \mu s$ Delay 


\section{Chapter 7}

\section{Conclusions}

A single-plate interferometric system capable of capturing unsteady passing shock events was successfully developed. This system incorporated advanced CCD technology to facilitate the "freezing" of a highly unsteady event, in this case that of a passing shock. Using this newly developed system, a study of the time history of the density field in a transonic turbine blade passage during a passing shock event was performed. The variations in the cascade inflow could be seen, not only during the initial shock passage, but during all of the subsequent reflections. A previous attempt made at performing an idealized analytical study of the passing shock event could only be performed up to the initial shock passing due to the large number variables in the system [17]. These measurements uniquely quantify this event and are a significant step in increasing our understanding of unsteady rotor/stator interactions with transonic turbine engines.

Optical methods hold a great advantage over traditional measurement systems in that there is little or no disturbance to the flow field. Also, the entire flow field can be studied simultaneously without having to resort to tedious pointwise measurement techniques. These factors are especially critical given the transonic flow conditions and small physical dimensions of this test section. In the case of a single-plate interferometric system, the disadvantage of requiring a portion of the density field to be know and the difficulties in inherent in the data reduction must be weighed against the simplicity of the setup. However, the area where the known reference density field 
is taken can be chosen to minimize the flow disturbances and increase the accuracy of the measurement.

The single-plate system did indeed prove simple to setup, a very important consideration when using shared facilities. The entire system setup could easily be performed in less than two hours, compared to the days a Mach-Zhender interferometer often requires. The replacement of the Polaroid film with the CCD camera proved advantageous in this regard. The internal shuttering mechanism of the CCD camera, removed the Bragg-cell from the previous optical system, greatly reducing the effort required in the interferometric system setup. Also, because of the extremely quick shutter times used while working with the intensified CCD camera, it was no longer necessary to capture the images in a darkened room. This made the task of operating the tunnel simpler.

Using a combination of high-frequency Kulite pressure transducers and custom hot wire probes, the required unsteady know density field was successfully recorded. In order not to disturb the flow in the blade passage, it was chosen to take the reference area upstream of the blade row. Timed shadowgraphs were used to choose shutter times where the shock wave was not situated within the studied density field, in order to improve the accuracy of the results. One problem that was encountered was the passage of Mylar upstream of the test section after the passage of the shock. These Mylar fragments had a tendency to break the hot wire. However, since these fragments traveled behind the passing shock wave, the shock event was completed before the hot wire lost continuity. However, since the number of required runs was high, the wire had to be replaced numerous times. This did tend to slow down the data collection.

The reduced density field plots demonstrated the capability of the system to track the passage of the shock wave and the numerous reflections in a simulation of a NGV and high pressure turbine rotor unsteady interaction. The expected pressure rise following the shock passage was shown in the density fields, and the effect of the passing shock on the inlet flow was clearly seen. Interferometric images have also been taken in the blade passage and wake region of the flowfield for the times presented above and are included in Appendix I. Data reduction on these images is waiting the 
development of more sophisticated software and data reduction algorithms.

Using a magnified spark-shadowgraph survey, the boundary layer was found to be laminar over both the pressure and suction surfaces of the blade. The laminar state of the suction surface can be attributed to the high flow acceleration experienced by the boundary layer over the entire blade surface up to the shock impingement. This finding was supported by a hydrodynamic stability analysis performed on the blade suction surface.

Since, in a typical engine environment the boundary layer is usually turbulent due to extremely high values of FST, film cooling and 3-D effects, it was necessary to force the boundary layer of our blades to become transitional. This is especially important if any heat flux studies are to be made, since heat transfer is greatly affected by the state of the boundary layer. Using the hydrodynamic stability analysis to tailor the frequency content of grid generated FST, it was found that the state of the boundary layer was not only dependent on the level, but also on the characteristics of the FST.

Areas for future work include:

1) Improving the image processing algorithms, allowing the vertical image shifting into the blade passage and wake regions.

2) Expansion of the study to evaluate the effect of multiple passing shock events. This would allow the quantitative study of the effect shock spacing (in time) has on the inlet flow and pressure rise of the density field.

3) Computational studies using the interferometric study for code validation. As stated before, one of the main uses of interferometric studies is providing a database of reliable data to help in the validation and improvement of CFD codes.

4) Determine the state of the boundary layer during the unsteady passing shock event. If CFD calculations are to be performed on this flowfield, it will be necessary to determine if the passing shock changes temporarily the state of the boundary layer. 


\section{Bibliography}

[1] L. Fottner. Review on turbomachinery blading design problems. Lecture Series AGARD-LS-167, AGARD, May 1989.

[2] S. V. Janakiraman. Fluid flow and heat transfer in transonic turbine cascades. Master's thesis, Virginia Polytechnic Institute and State University, May 1993.

[3] D. J. Doorly and M. L. G. Oldfield. Simulation of the effects of shock wave passing on a turbine rotor blade. Transactions of the ASME, 107:998-1006, Oct 1985.

[4] K. V. Rao, R. A. Delaney, and M. G. Dunn. Vane-blade interaction in a transonic turbine, part 1: Aerodynamics. Journal of Propulsion and Power, 10(3):305-311, 1994.

[5] H. E. Gallus. Unsteady Aerodynamic Measurments on Rotors. AGARD, 1987. AGARDograph No. 298.

[6] P. F. Bry. Blading design for cooled high-pressure turbines. Lecture Series AGARD-LS-167, AGARD, May 1989.

[7] T. Kiss. Experimental and Numerical Investigation of Transonic Turbine Cascade Flow. PhD thesis, Virginia Polytechnic Institute and State University, Dec 1992.

[8] H. Pfeil, R. Herbst, and T. Schröder. Investigation of the laminar-turbulent transition of boundary layers disturbed by wakes. ASME Journal of Turbomachinery, 105:130-137, 1983. 
[9] F. J. Bayley and W. J. Priddy. Effects of free-stream turbulence on the distribution of heat transfer around turbine blade sections. International Journal of Heat Fluid Flow, 6(3):181-191, 1985.

[10] D. J. Doorly and M. L. G. Oldfield. Simulation of wake passing in a stationary turbine rotor cascade. Journal of Propulsion, 1(4):316-318, Jul and Aug 1985.

[11] D. L. Schultz, A. B. Johnson, D. A. Ashworth, M. J. Rigby, and J. E. LaGraff. Wake and shock interactions in a transonic turbine stage. In AGARD 68th Specialists' Meeting, Transonic and Supersonic Phenomena in Turbomachines, number 3, 1986.

[12] D. A. Ashworth, J. E. LaGraff, and D. L. Schultz. Unsteady interaction effects on a transitional turbine blade boundary layer. Journal of Turbomachinery, 111:162-168, Apr 1989.

[13] S. U. Patankar and D. B. Spalding. Heat and Mass Transfer in Boundary Layers. Intertext Book, London, 2 edition, 1970.

[14] A. Forest. Engineering predictions of transitional boundary layers. In $A G A R D$ Conference Proceedings, Laminar-Turbulent Transition, number CP-224, 1977. Paper No. 22.

[15] A. B. Johnson, M. L. G. Oldfield, M. J. Rigby, and M. B. Giles. Nozzle guide vane shock wave propagation and bifuration in a transonic turbine cascade. Technical Report 90-GT-310, ASME, 1990.

[16] J. C. Collie, H. L. Moses, J. A. Schetz, and B. A. Gregory. Recent advances in simulating unsteady flow phenomena brought about by passage of shock waves in a linear turbine cascade. Journal of Turbomachinary, 115:687-698, Oct 1993.

[17] R. L. Doughty. Effects of Multiple Incident Shock Waves on the Flow in a Transonic Turbine Cascade. PhD thesis, Virginia Polytechnic Institute and State University, Dec 1994.

[18] J. P. Gostelow. Cascade Aerodynamics. Pergamon Press, 1984. 
[19] J. J. Camus, J. D. Denton, J. V. Soulis, and C. T. J. Scrivener. An experimental and computational study of transonic three-dimensional flow in a turbine cascade. Journal of Engineering for Gas Turbines and Power, 106:414-420, Apr 1984.

[20] Personal communication with D. Holmberg.

[21] D. L. Merritt and P. M. Aronson. Study of blast-bow wave interactions in a wind tunnel. AIAA Paper, (65-5), 1965.

[22] K. Takayama. Shock tube and shock wave research. In Proceedings of the Eleventh International Symposium on Shock Tubes and Waves, pages 74-81, Jul 1977.

[23] Personal communication with T. Reid.

[24] D. W. Holder, R. J. North, and G. P. Wood. Optical Methods for Examining the Flow in High-Speed Wind Tunnels. North Atlantic Treaty Organization Advisory Group for Aeronautical Research and Development, Nov 1956.

[25] J. E. Harris and D. K. Blanchard. Computer Program for Solving Laminar, Transitional or Turbulent Compressible Boundary-Layer Equations for TwoDimensional and Axisymmetric Flow, 1982. NASA TM-83207.

[26] V Iyer. Computer Program BL2D for Solving Two-Dimensional and Axisymmetric Boundary Layers, MAY 1995. NASA CR-4668.

[27] J. A. Schetz. Boundary Layer Analysis. Prentice Hall, Englewood Cliffs, NJ, 1993.

[28] L. Lees and C. C. Lin. Technical Report NACA-TN-1115, NACA, 1946.

[29] M. R. Malik. Journal of Computational Physics, 86:376, 1990.

[30] M. R. Malik and R. E. Spall. On the stability of compressible flow past axisymmetric bodies. Journal of Fluid Mechanics, 228:443-463, 1991. 
[31] M. F. Blair. Influence of free-stream turbulence on boundary layer transition in favorable pressure gradients. Journal of Engineering For Power, 104:743-750, Oct 1982 .

[32] L. P. Johnson. Effects of free-stream turbulence on turbine blade heat transfer in transonic flow. Master's thesis, Virginia Polytechnic Institute and State University, 1995.

[33] T. Kinoshita and D. R. Yennie. High precision tests of quantum electrodynamics - an overview. In T. Kinoshita, editor, Quantum Electrodynamics, volume 7 of Advanced Series on Directions in High Energy Physics. World Scientific, Singapore, 1990.

[34] A. J. Lightman and S. Cartwright. Techniques for optical interferanemography. Technical Report AFWAL-TR-85-3098, Air Forcu Wright Aeronautical Laboratories, Dec 1985.

[35] F. L. Pedrotti and L. S. Pedrotti. Introduction to Optics. Prentice-Hall, Englewood Cliffs, NJ, 1987.

[36] M. M. Blouke. Charge-coupled devices. In Encyclopedia of Applied Physics, volume 3, pages 241-271. VCH, 1992.

[37] R. A. Sturz. Advances in low level video imaging. In Airborne Reconnaissance XIX, volume 2555, pages 196-206. SPIE, Jul 1995.

[38] B. Walder. Personal Fax, Feb 1994.

[39] D. C. O'Shea, W. R. Callen, and W. T. Rhodes. Introduction to Lasers and Their Applications. Addison-Wesley, Reading, Mass, 1977.

[40] A. J. Smits, K. Hayakawa, and K. C. Muck. Constant temperature hot-wire anemometer practice in supersonic flows. In Experiments in Fluids, volume 1, pages 83-92, 1983.

[41] C. A. Lindley. Pratical Image Processing In C. Wiley, New York, 1991. 
[42] Ø. D. Trier and T. Taxt. Evaluation of binarization methods for document images. In IEEE Transaction on Pattern Analysis and Machine Intelligence, volume 17, pages 312-315, Mar 1995.

[43] L. J. Bush. Evaluating the accuracy of line thinning algorithms after processing scanned line data. Master's thesis, Virginia Polytechnic Institute and State University, 1993.

[44] J. H. Wilkinson. The Algebraic Eigenvalue Problem. Clarendon Press, Oxford, 1965.

[45] R. L. Burden and J. D. Faires. Numerical Analysis. PWS, Boston, Mass, 5 edition, 1993. 


\section{Appendix A QR Algorithm}

There are a large variety of methods available for the calculation of eigenvalues and their associated eigenvectors. These include the Power method, deflation methods and the QR method. Since each of these methods have certain advantages and disadvantages, the most suitable algorithm will be dependent on the application.

The power method only provides a solution to the dominant eigenvalue and the associated eigenvector so it is used in conjunction with deflation techniques. Deflation techniques provide a solution for all other eigenvalues once the dominant eigenvalue is known. However, this method is generally not suitable for calculating all of the eigenvalues in a matrix. The growth of round-off error due to the large number of iterations required in this algorithm.

More often implemented are matrix reduction techniques where all of the eigenvalues are determined simultaneously. One such algorithm is the QR algorithm, often used due to its excellent stability characteristics. Here I will outline its application to symmetric matrices. For a full explanation of this algorithms application to non-symmetric matrices, see Wilkinson [44].

The $\mathrm{QR}$ algorithm requires a tridiagonal, symmetric matrix as input to the matrix equations. Therefore, if the original symmetric matrix is not tridiagonal, one must be found that is similar to the initial symmetric matrix, $\mathbf{A}$.

Using the following theorem [45]:

If $\mathbf{A}$ is an $n x n$ symmetric matrix and $\mathbf{D}$ is a diagonal matrix whose diagonal entries are the eigenvalues of $\mathrm{A}$, then there exists an orthogonal matrix $\mathrm{P}$ such that

$$
[\mathbf{D}]=[\mathbf{P}]^{-1}[\mathbf{A}][\mathbf{P}]=[\mathbf{P}]^{t}[\mathbf{A}][\mathbf{P}]
$$

Equation A.1 implies that $\mathbf{A}$ is similar to a diagonal matrix $\mathbf{D}$, since an orthogonal matrix 
$\mathbf{Q}$ exists with the property that $\mathbf{D}=\mathbf{Q}^{-1} \mathbf{A Q}$.

Since, in general, the matrix $\mathbf{Q}$ is difficult to compute, the Householder method provides an approximate matrix $\mathbf{Q}$ using the following transformation:

Let $\mathbf{w} \in \Re^{n}$ with $\mathbf{w}^{t} \mathbf{w}=1$. The Householder transformation $n x n$ matrix is then

$$
[\mathbf{d P}]=[\mathbf{I}]-2 \mathbf{w} \mathbf{w}^{t}
$$

The Householder transformation selectively zeros out blocks of entries in columns of matrices.

Thus,

$$
[\mathbf{A}]^{(n-1)}=[\mathbf{P}]^{(n-2)}[\mathbf{P}]^{(n-3)} \ldots \ldots \ldots \ldots[\mathbf{P}]^{(1)}[\mathbf{A}][\mathbf{P}]^{(1)} \ldots \ldots \ldots . .[\mathbf{P}]^{(n-3)}[\mathbf{P}]^{(n-2)}
$$

Now, if we denote $\mathbf{A}$ as the tridiagonal, symmetric matrix, the following notation can be used.

$$
[\mathbf{A}]=\left[\begin{array}{cccccc}
a_{1} & b_{2} & 0 & \cdots & \cdots & 0 \\
b_{2} & a_{2} & b_{3} & \ddots & & \vdots \\
0 & b_{3} & a_{3} & \ddots & \ddots & \vdots \\
\vdots & \ddots & \ddots & \ddots & \ddots & 0 \\
\vdots & & \ddots & \ddots & \ddots & b_{n} \\
0 & \cdots & \cdots & 0 & b_{n} & a_{n}
\end{array}\right]
$$

Thus, if $b_{2}=0$ or $b_{n}=0$, then $a_{1}$ or $a_{n}$ is an eigenvalue of [A]. If $b_{j}=0$ for some $\mathrm{j}$, where $2<j<n$, the matrix can be divided into two smaller matrices given by:

$$
\left[\begin{array}{cccccc}
a_{1} & b_{2} & 0 & \cdots & \cdots & 0 \\
b_{2} & a_{2} & \ddots & \ddots & & \vdots \\
0 & \ddots & \ddots & \ddots & \ddots & \vdots \\
\vdots & \ddots & \ddots & \ddots & \ddots & 0 \\
\vdots & & \ddots & \ddots & \ddots & b_{j-1} \\
0 & \cdots & \cdots & 0 & b_{j-1} & a_{j-1}
\end{array}\right] \text { and }\left[\begin{array}{cccccc}
a_{j} & b_{j+1} & 0 & \cdots & \cdots & 0 \\
b_{j+1} & a_{j+1} & \ddots & \ddots & & \vdots \\
0 & \ddots & \ddots & \ddots & \ddots & \vdots \\
\vdots & \ddots & \ddots & \ddots & \ddots & 0 \\
\vdots & & \ddots & \ddots & \ddots & b_{n} \\
0 & \ldots & \cdots & 0 & b_{n} & a_{n}
\end{array}\right]
$$

If no zeros can be found, the algorithm proceeds by forming a sequence of matrices $\mathbf{A}=\mathbf{A}^{(1)}, \mathbf{A}^{(2)}, \mathbf{A}^{(3)}, \ldots$ 
These matrices are calculated with the following method. First,

$$
[\mathbf{A}]^{1}=[\mathbf{A}] \quad \text { is factored as a product } \quad[\mathbf{A}]^{1}=[\mathbf{Q}]^{1}[\mathbf{R}]^{1}
$$

where $\mathbf{Q}^{(1)}$ is orthogonal and $\mathbf{R}^{(1)}$ is upper triangular. Refer to Burden [45] to see how a rotation matrix can be used to factor $\mathbf{A}$.

$\mathbf{A}^{(2)}$ is now defined as

$$
[\mathbf{A}]^{2}=[\mathbf{R}]^{1}[\mathbf{Q}]^{1}
$$

which can be factored again as the product

$$
[\mathbf{A}]^{2}=[\mathbf{Q}]^{2}[\mathbf{R}]^{2}
$$

Thus, in general,

$$
[\mathbf{A}]^{i}=[\mathbf{Q}]^{i}[\mathbf{R}]^{i}
$$

Also, since $\mathbf{Q}^{i}$ is orthogonal,

$$
[\mathbf{A}]^{i+1}=[\mathbf{R}]^{i}[\mathbf{Q}]^{i}=\left([\mathbf{Q}]^{i^{t}}[\mathbf{A}]^{i}\right)[\mathbf{Q}]^{i}=[\mathbf{Q}]^{i^{t}}[\mathbf{A}]^{i}[\mathbf{Q}]^{i}
$$

where $\mathbf{A}^{(i+1)}$ is a symmetric, tridiagonal matrix with the same eigenvalues as $\mathbf{A}^{(i)}$ and thus the same eigenvalues as the original matrix $\mathbf{A}$. 


\section{Appendix B}

\section{Avg01.cpp}

//Program Name: Avg01.cpp

$1^{*}$ This program allows a series of averages to be calculated on up to ten data files. */

\#include $<$ fstream.h $>$

\#include $<$ iostream.h $>$

\#include<iomanip.h $>$

\#include $<$ stdio.h $>$

\#define true 1;

\#define false 0;

ifstream InFile01;

ifstream InFile02;

ifstream InFile03;

ifstream InFile04;

ifstream InFile05;

ifstream InFile06;

ifstream InFile07;

ifstream InFile08;

ifstream InFile09;

ifstream InFile10;

ifstream InFile90;

ifstream InFileA; 
ofstream Output;

void FilePrimer(int\& n);

void PointAverage(int $\mathbf{n}$, char filename[]);

void GetName(char filename[], int out);

void FileAverage(char filename[]);

int TestFiles(int $\mathrm{n}$ );

void DumpToFile();

void FilePrimer(int\& $\mathbf{n})$

//FilePrimer initializes the input streams for input. It allows up to ten //separate input files to be accessed simultaneously. These are global //variables, and as such don't have to be passed back and forth among the //subroutines. The only value which the function returns is the number //of files which are opened.

$\{/ /$ FilePrimer

char filename[50];

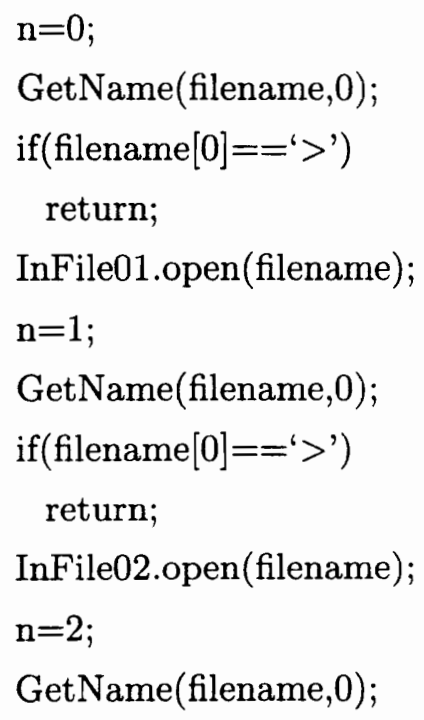




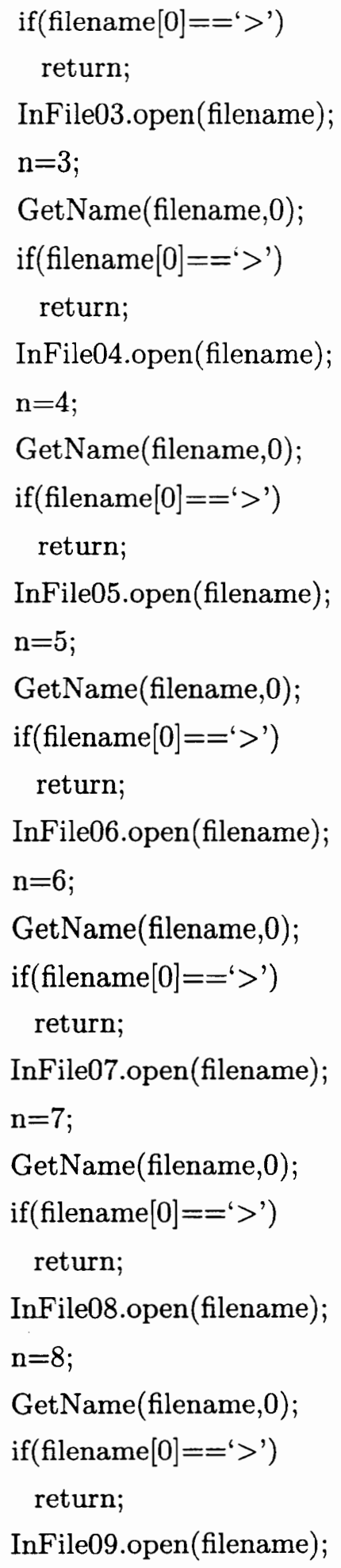


$\mathrm{n}=9$;

GetName(filename,0);

if(filename $[0]=={ }^{\prime}>$ ')

return;

InFile10.open(filename);

$\mathrm{n}=10$;

return;

\}//FilePrimer

void PointAverage(int $n$,

char filename[])

//This procedure takes the value at a given time coordinate in the //separate files and then averages them. It takes this average and //outputs it to a user defined file.

$\{/ /$ PointAverage

int in,period,times;

double Sum,Average,value;

in = true;

period $=0$;

times $=0$;

cout $<<$ "Processing" $<<$ endl;

Output.open(filename);

while(in)

$\{/ /$ while not end of file

Sum $=0.0$

period++;

if $($ period $==1000)$

\{

times++ ;

if $($ times $==40)$

\{ 


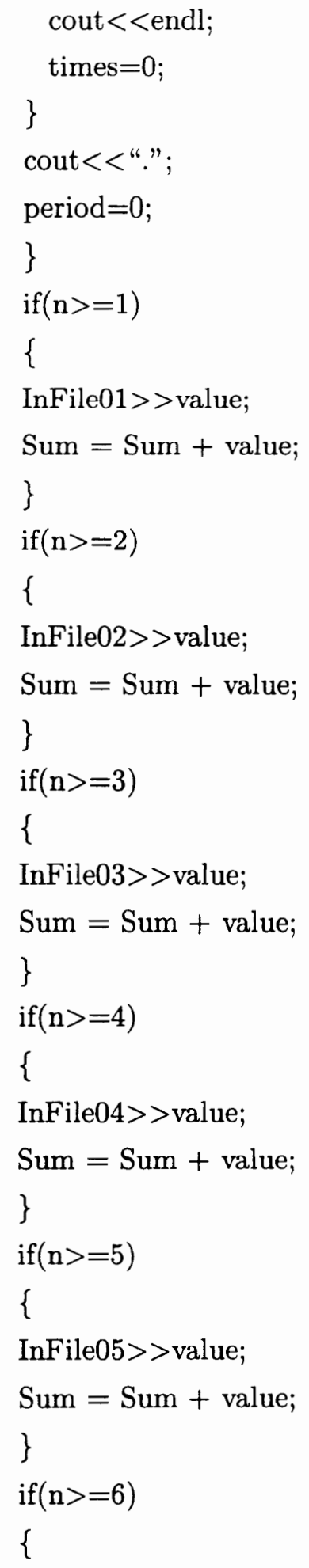




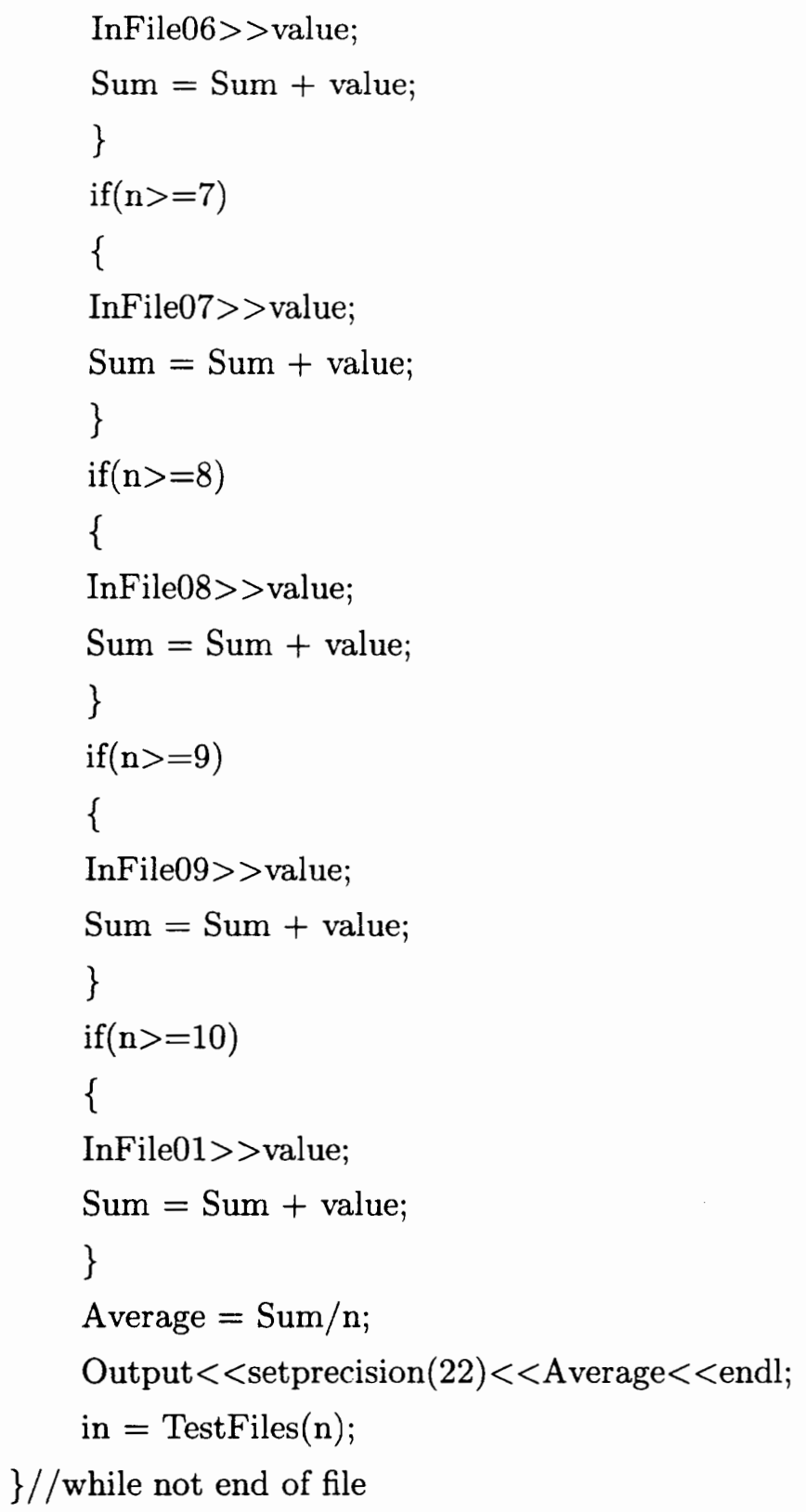

void GetName(char filename[], 
int out)

//This subroutine gets the file name from the user

$\{/ /$ GetName

int done $=$ false;

while(!done)

$\{/ /$ while not done

cout $<<$ "Enter file name:" $<<"$ ";

cin $>>$ filename;

cout $<<$ endl;

InFile90.open(filename);

done $=$ TestFiles $(-90)$;

InFile90.close();

if $\left(\left(\right.\right.$ filename$[0]==^{\prime}>$ ' $)-($ out $\left.)\right)$ done $=$ true;

if(!done) cout $<<$ "file does not exist" $<<$ endl;

\}$/ /$ while not done

\}//GetName

void FileAverage(char filename[])

//This function opens an input file specified in the call and then averages

$/ /$ the values present in that function

$\{/ /$ FileAverage

long $\mathbf{n}=0$;

double Sum, Value;

Sum $=0.0$

InFileA.open(filename);

while(InFileA)

$\{/ /$ while in file

$$
\mathrm{n}++ \text {; }
$$


InFileA $>>$ Value;

Sum = Sum + Value;

\}$/ /$ while in file

cout $<<$ "The File average is: " $<<$ setprecision $(22)<<(\mathrm{Sum} / \mathrm{n})<<$ endl; $\} / /$ FileAverage

int TestFiles(int $\mathrm{n}$ )

//This procedure tests to see if all the files are still accessable

//A zero is returned if all of them are open, and a one is returned

$/ /$ if any are finished.

$\{/ /$ TestFiles

int test $=0$;

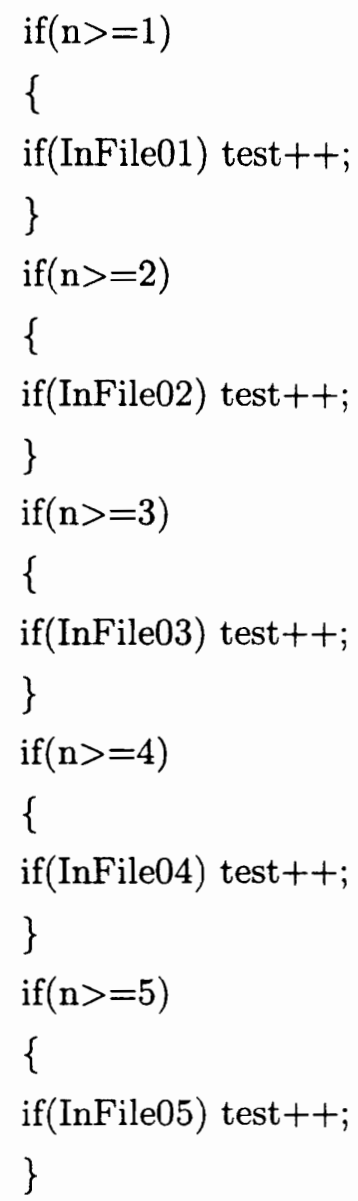




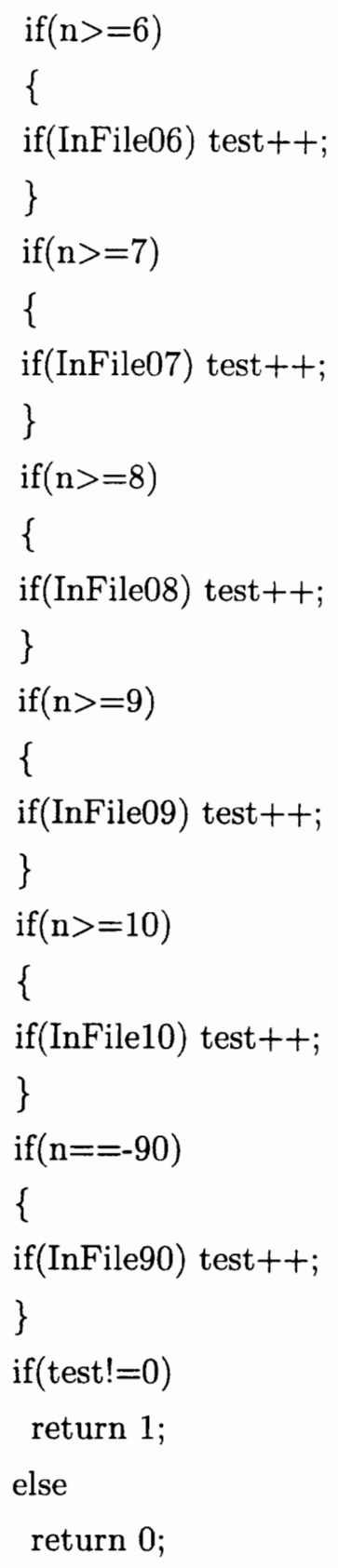

//Dumps the contents of the temporary file into a real user file 
$\{/ /$ DumpToFile

char filename[50];

double Buffer;

cout $<<$ "For output, ";

GetName(filename,1);

InFileA.open( "avgtmp.dat");

Output.open(filename);

while(InFileA)

$\{/ /$ while in InFileA

InFileA $>>$ Buffer;

Output $<<$ Buffer;

\}$/ /$ while in InFileA

Output.close();

InFileA.close();

\}//DumpToFile

void main()

$\{/ /$ main

int Done, menu,n;

char filename[50];

Done $=$ false;

while(!Done)

$\{/ /$ while not done

$\mathrm{n}=0$;

cout $<<$ "Main Menu" < <endl $<<$ "1. Unsteady Average" $<<$ endl $<<$

"2. Upstream Average" $<<$ endl $<<$ "3. Wake Average" $<<$ endl $<<$ "4.Quit" $<<$ endl;

$\operatorname{cin}>>$ menu; 
$\operatorname{switch}(\mathrm{menu})$

$\{/ /$ switch

case 1: FilePrimer(n);

if $(n==0)$ break;

cout<<"For output, ";

GetName(filename,1);

Point Average(n,filename);

break;

case 2: GetName(filename,0);

FileAverage(filename);

break;

case 3: FilePrimer(n);

if $(\mathrm{n}==0)$ return;

PointAverage(n, "avgtmp.dat");

DumpToFile();

FileAverage( "avgtmp.dat");

break;

case 4: Done = true;

break;

default: Done=false;

\}//switch

remove("avgtmp.dat");

InFile01.close();

InFile02.close();

InFile03.close();

InFile04.close();

InFile05.close();

InFile06.close();

InFile07.close();

InFile08.close();

InFile09.close();

InFile10.close();

InFileA.close(); 
InFile90.close();

Output.close();

cout $<<$ endl $<<$ endl $<<$ endl;

\}$/ /$ while not done

\}$/ /$ main 


\section{Appendix C}

\section{ensavg.cpp}

//Program Name: ensavg.cpp

$/^{*}$ This program runs an ensamble average on up to five data files which are specified by the user. All data is stored and process in double precision dynamic arrays and output to the user defined file at double precision. */

\#include $<$ fstream.h $>$

\#include $<$ iostream.h $>$

\#include<iomanip.h $>$

\#include $<$ stdio.h $>$

\#define true 1 ;

\#define false 0 ;

const int NumRows $=5$;

const int NumCols $=132000$;

const int NumTrigPts $=66000$;

typedef double* trace; //pointer to double for trace arrays

typedef int StartArray[NumRows];

typedef trace TArray[5]; //an $\mathrm{x}$ by 5 array of pointers

void InputArray(int\& NumFiles, TArray TraceData, StartArray Zero); 
void SetTrigger(int NumTrace, TArray TraceData, StartArray ZeroPt);

void PointAverage(int NumTrace, TArray TraceData, trace Average,

StartArray ZeroPt);

void Output(trace FO);

void Getname(char filename[]);

double RowAvg(int Index, TArray TraceData);

void PreTrigAvg(int NumTrace, TArray TraceData,trace Average,

StartArray ZeroPt,int\& AvgIndex);

void InputArray(int\& NumFiles,

TArray TraceData,

StartArray Zero)

$/ *$ InputArray reads in the data from up to five user specified

files and then returns that data in the TraceData array. The

function also returns the number of files that were read

into the array. $* /$

$\{/ /$ InputArray

int ColIndex,x;

trace TrigData[5];

ifstream InFile;

char filename[25];

for $(x=0 ; x<5 ; x++)$

\{

TrigData[x] $=$ new double[NumTrigPts]; //create dynamic array for(int $\mathrm{i}=0 ; \mathrm{i}<$ NumTrigPts; $\mathrm{i}++$ )

\{

TrigData $[\mathrm{x}][\mathrm{i}]=0 ; / /$ initialize each new array element to zero

\}

\}

for $($ NumFiles $=0 ;$ NumFiles $<$ NumRows;NumFiles ++ ) 
$\{/ /$ for NumFiles

Getname(filename);

if (filename $[0]==$ '>')

$\{/ /$ if filename is " $>$ "

NumFiles-; //since this isn't a file counts back \# files by one

break; //forces out of for Numfiles loop

\}$/ /$ if filename is " $>$ "

InFile.open(filename);

ColIndex $=0$;

while(InFile)

$\{/ /$ while InFile

InFile $>>$ TraceData[NumFiles][ColIndex];

Collndex++;

\}//while InFile

InFile.close();

cout $<<$ "For the respetive trace, ";//need trigger data to calculate

Getname(filename); //the location of the first shock

InFile.open(filename);

ColIndex $=0$;

while(InFile)

\{

InFile $>>$ TrigData[NumFiles][ColIndex];

ColIndex++;

\}

InFile.close();

\}//for NumFiles

SetTrigger(NumFiles,TrigData,Zero);

delete [ TrigData;

\}//InputArray

void SetTrigger(int NumTrace,

trace TrigData[], 


\section{StartArray ZeroPt)}

$/{ }^{*}$ SetTrigger determines the starting point for the ensemble average.

This point is located at the begining of the first shock. In

order to make this feasible, the assumption is made that the first shock will be where there is a value $20 \%$ higher than the average voltage reading. Therefore, upon coming across a value which is $20 \%$ higher, the procedure will assume that this is the begining of the first shock and will then store that number as the zero point in the ZeroPt array */

\section{$\{/ /$ SetTrigger}

int TraceIndex,ShockSearch,FirstShock;

double AverageVoltage;

\section{AverageVoltage $=0$}

for $($ TraceIndex $=0 ;$ TraceIndex $<=$ NumTrace $;$ TraceIndex ++ )

$\{/ /$ for TraceIndex

AverageVoltage $=$ RowAvg $($ TraceIndex, TrigData $)$;

FirstShock=false;

for (ShockSearch=0;(ShockSearch $<$ NumTrigPts)\&\&(!FirstShock);ShockSearch ++$)$

$\{/ /$ for ShockSearch

if(TrigData[TraceIndex][ShockSearch] $>\left(1.2^{*}\right.$ AverageVoltage $\left.)\right)$

$\{/ /$ if TraceData

FirstShock=true;

cout $<<$ "New shock location is: "<<ShockSearch $<<$ " and @:";

cout $<<$ TrigData[TraceIndex][ShockSearch] $<<$ endl;

ZeroPt $\left[\right.$ TraceIndex] $=$ ShockSearch ${ }^{*} 2 ; / /{ }^{*} \mathrm{~b} / \mathrm{c}$ of diff. sample rate

\}//if TraceData

\}//for ShockSearch

\}//for TraceIndex

\}//SetTrigger 
void PointAverage(int NumTrace,

TArray TraceData,

trace Average,

StartArray ZeroPt)

$/{ }^{*}$ Point Average does the ensamble averaging. It calls the function

PreTrigAvg to average the points before the trigger, but then

averages the points after the trigger itself. These are all

put into an EnsArray. */

$\{/ /$ Point Average

int $\mathrm{x}$,Done, $\mathrm{n}$, AvgIndex, $\mathrm{u}$;

double Sum;

StartArray Index;

Done $=$ false

AvgIndex $=0 ; / /$ AvgIndex keeps track of location in Average Array

Sum $=0$;

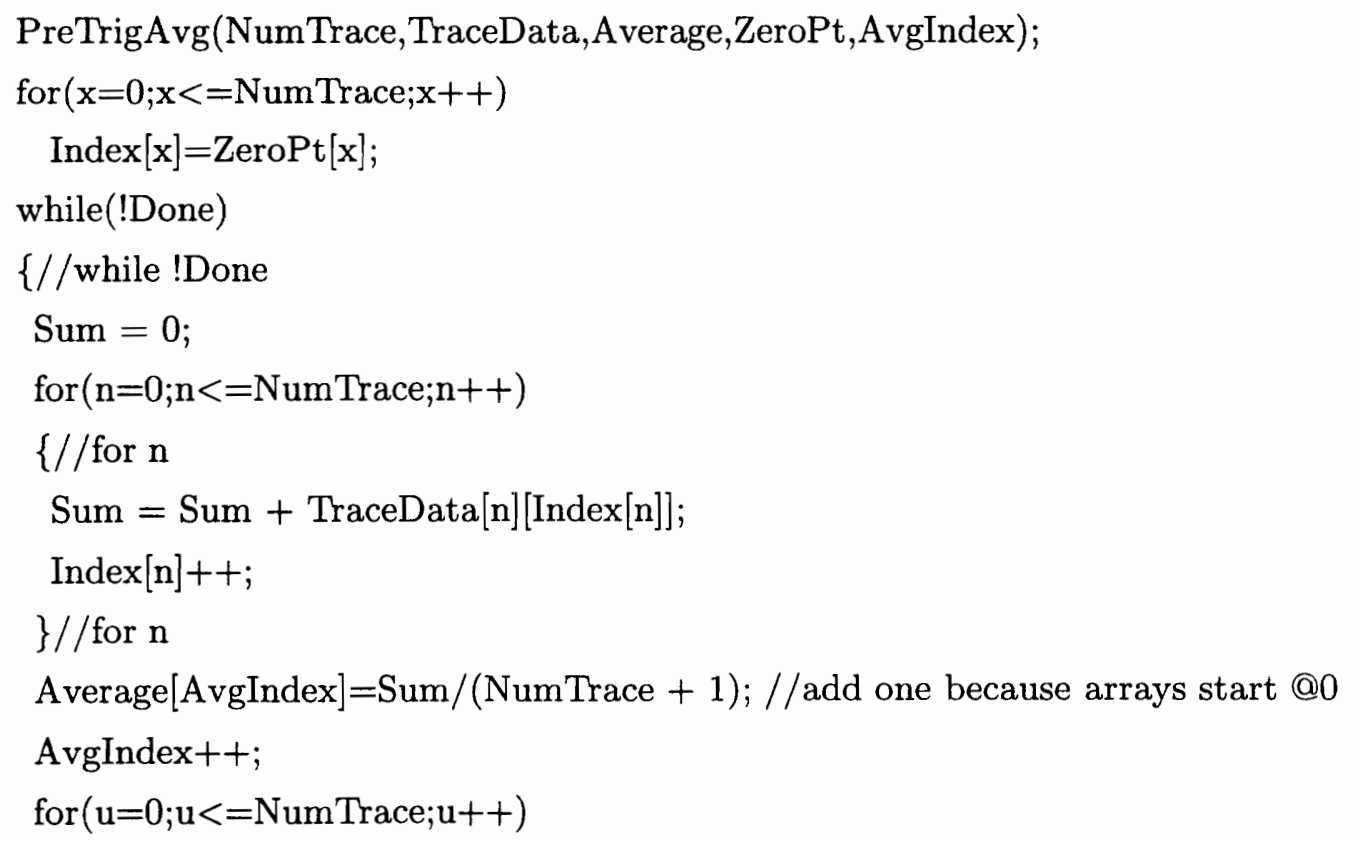




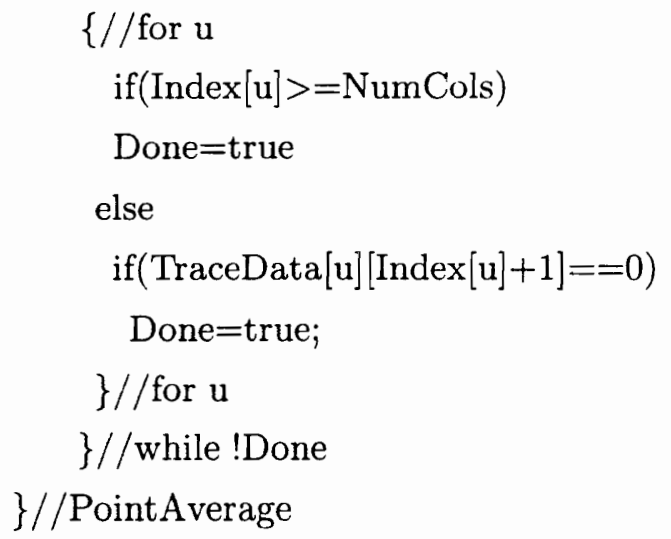

void Getname(char flename[]) 
$/{ }^{*}$ Getname gets the file name from the user and determines whether or not this is a current file. If it is not, it prompts the user for a new file name. In the event that a ">" is entered, the check is not performed and the function is terminated. */

$\{/ /$ GetName

int done;

ifstream TestIn;

do

$\{/ /$ while not done

cout $<<$ "Enter the filename: ";

cin $>>$ filename;

TestIn.open(filename);

if(TestIn)

done=true;

if (filename $[0]=={ }^{\prime}>$ ')

done=true;

if(!done)

cout $<<$ "file does not exist" $<<$ endl;

\}while(!done);

TestIn.close();

\}//Getname

double RowAvg(int Index, trace TrigData[])

$/{ }^{*}$ RowAvg takes the average of a certain trace and returns that value through the header. */

$\{/ /$ Row Avg

int $\mathrm{x}$, count;

int End =false; 
double Sum=0;

count $=0$

for $(\mathrm{x}=0 ;(\mathrm{x}<$ NumTrigPts $)-($-End $) ; \mathrm{x}++)$

$\{/ /$ for $\mathrm{x}$

Sum $=$ Sum + TrigData $[$ Index $][\mathrm{x}] ;$

if $($ TrigData[Index $][\mathrm{x}]==0) / /$ a zero means that there are no more

End=true; //data points even though there are more

count++; //points in the array.

\}//for $\mathrm{x}$

return(Sum/count);

\}//RowAvg

void PreTrigAvg(int NumTrace,

TArray TraceData,

trace Average,

StartArray ZeroPt,

int\& AvgIndex)

$/^{*}$ PreTrigAvg deals with the data averaging before the trigger this procedure reads the averages into a buffer, which is then read into the final array backwards. This must be done since the number of pre-trigger points in each trace are different. */

$\{/ /$ PreTrigAvg

int Done,n,Fill,BufIndex, u;

trace AvgBuffer; //buffers the average values to be sent back

StartArray Index $=\{0\}$;

double Sum;

Done $=$ false;

BufIndex $=0$; 


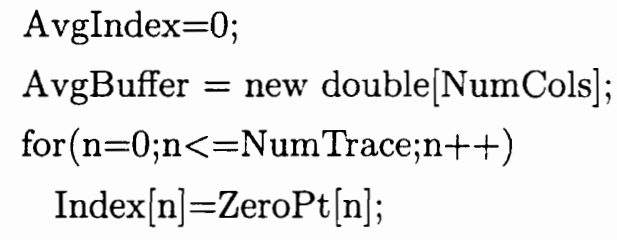

void main()

$\{/ /$ main

StartArray ZeroPt $=\{0\} ; / /$ stores the location of first shock for each array 
TArray TraceData; //array of pointers to keep track of signal data int NumTrace $=0 ; / /$ number of files loaded in to be averaged trace Average; //the ensamble average of the shocks

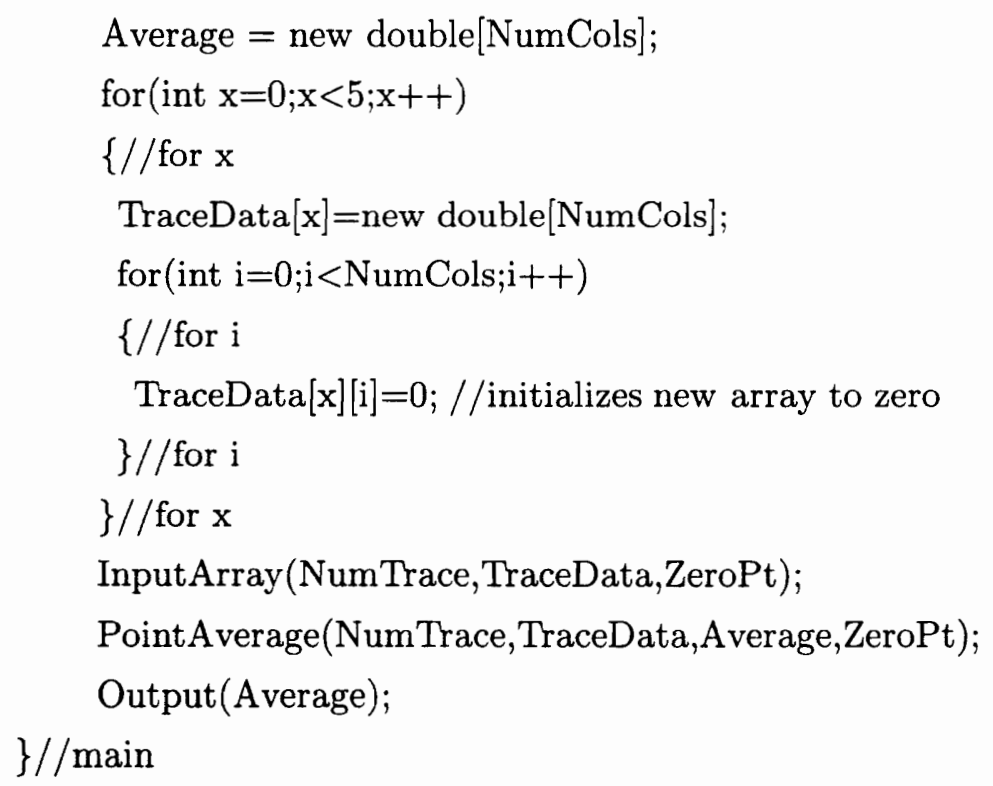




\title{
Appendix D
}

\section{Fdz.m}

\author{
\%Filename: Fdz.m
}

\section{\%Filetype: Matlab script}

\%this scipt is used to help in our filter design

$[b, a]=$ butter $(17,0.1) ; \%$ sets up butterworth filter array

$\mathrm{xf}=$ filter $(\mathrm{b}, \mathrm{a}, \mathrm{x}) ; \%$ runs butterworth filter on data and stores in $\mathrm{xf}$

cor $=$ cohere $(x, x f, 256,5000) ; \%$ checks for the coherence of the data

subplot $(2,1,1)$,plot(x) \%plots original signal

\%axis $([0,8000,3.5,5])$

$\operatorname{subplot}(2,1,2), \operatorname{plot}(\mathrm{xf})$

\%axis $([0,8000,3.5,5]) \%$ plots filtered signal

save temp.fil xf -ascii -double \%saves data in file "temp.fil"

clear all \%clears all variable buffers 


\title{
Appendix E \\ FringeFinal.cpp
}

\author{
// Program FringeFinal.cpp
}

$/^{*}$ This program performs the following algorithms on a raw CCD camera file and outputs the thinned image.*/

\#include <iostream.h $>$

\#include<math.h $>$

\#include $<$ stdio.h $>$

\#include $<$ stdlib.h $>$

const unsigned short WIDTH $=512$;

const unsigned short HEIGHT $=480$;

const unsigned short true $=1$;

const unsigned short false $=0$;

typedef unsigned char IAType[WIDTH][HEIGHT];

short xcount $=0$;

short ycount $=0$;

double Sum=0;

double $\mathrm{Mu}=0$;

short xstep, ystep; 
double RetValue $=0$;

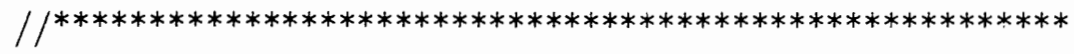

// define the functions

void ReadArray(IAType image);

void LowPass(short N,IAType image, IAType buffer);

void Binarization(short N, IAType image, IAType buffer);

void Output(IAType image);

double Integrate(short N, IAType image);

void Thinning(IAType image);

void BinaryToChar(IAType convert);

void CharToBinary(IAType convert);

void deleteS(IAType del);

void Invert(IAType convert);

int crossing (unsigned char cross[8]);

void ScaleImage(IAType image,IAType M, unsigned int SCol, unsigned int SRow, unsigned int SWidth, unsigned int

SHeight, double ScaleH, double ScaleV, unsigned int DCol, unsigned int DRow, unsigned int Interpolate,int $\mathrm{h} 1 \mathrm{x}$, int $\mathrm{v} 1 \mathrm{y}$ );

void MaskImage(IAType image,IAType buffer,int OriginY, int OriginX,int $r$,int $h 1 x$,int $v 1 y$ );

void ShiftImage(IAType image, IAType buffer, int $\mathrm{h} 1 \mathrm{x}$, int v1y);

$/ / * * * * * * * * * * * * * * * * * * * * * * * * * * * * * * * * * * * * * * * * * * * * * * * * * * * * * * * * * * * * *$

// BEGIN FUNCTIONS

/ / $* * * * * * * * * * * * * * * * * * * * * * * * * * * * * * * * * * * * * * * * * * * * * * * * * * * * * * * * * * * * *$

void ReadArray(IAType image)

$\{/ /$ ReadArray 


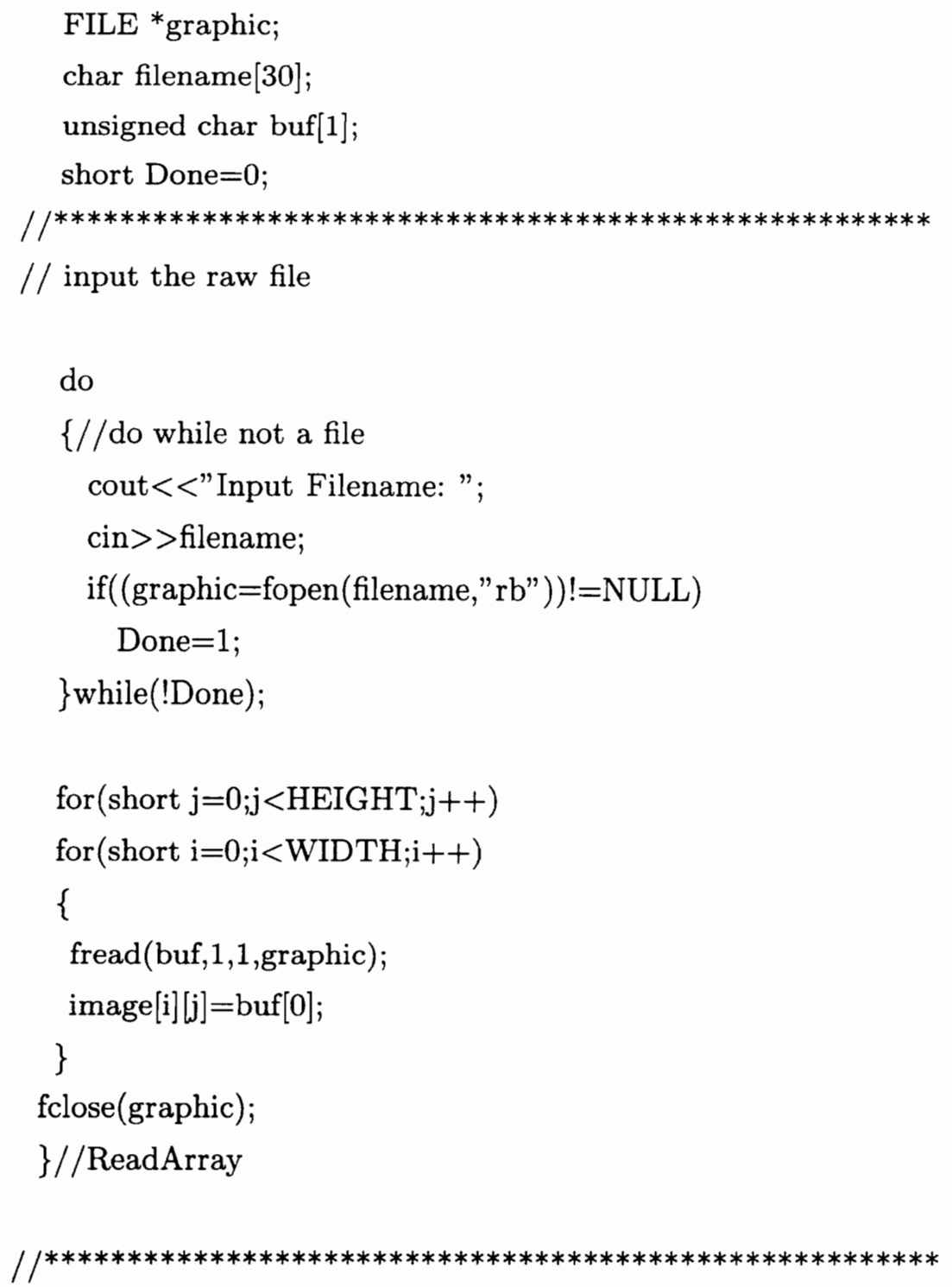

$/ /$ this function performs the low pass filter on the // CCD gray scale image

void LowPass(short N,IAType image, IAType M)

$\{/ /$ LowPass

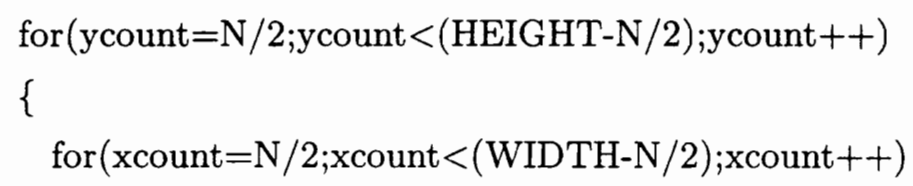




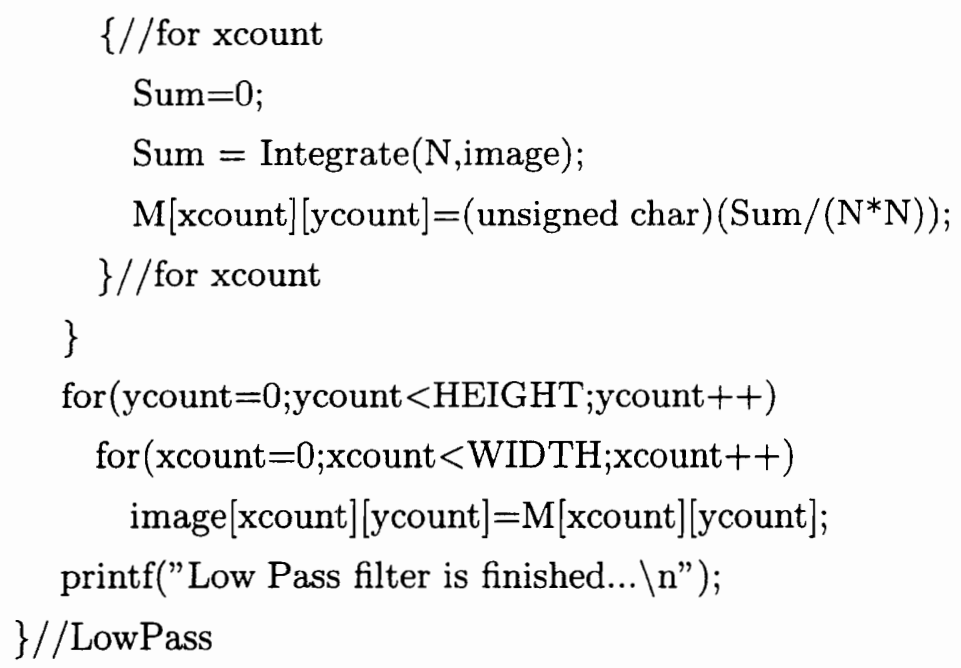

$/ /$ this function performs the adaptive binarization

void Binarization(short N, IAType image, IAType ImgBuf)

$\{/ /$ Binarization

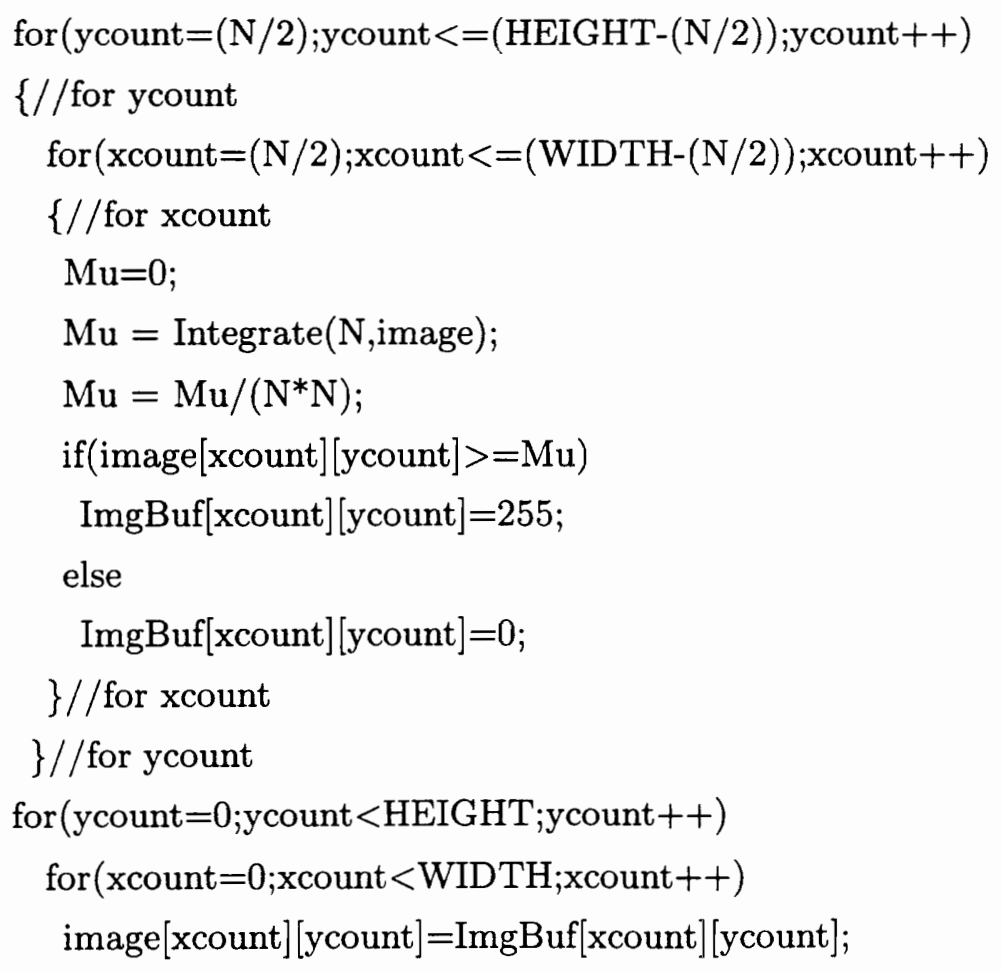




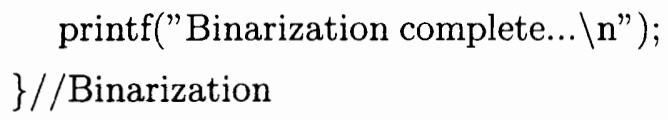




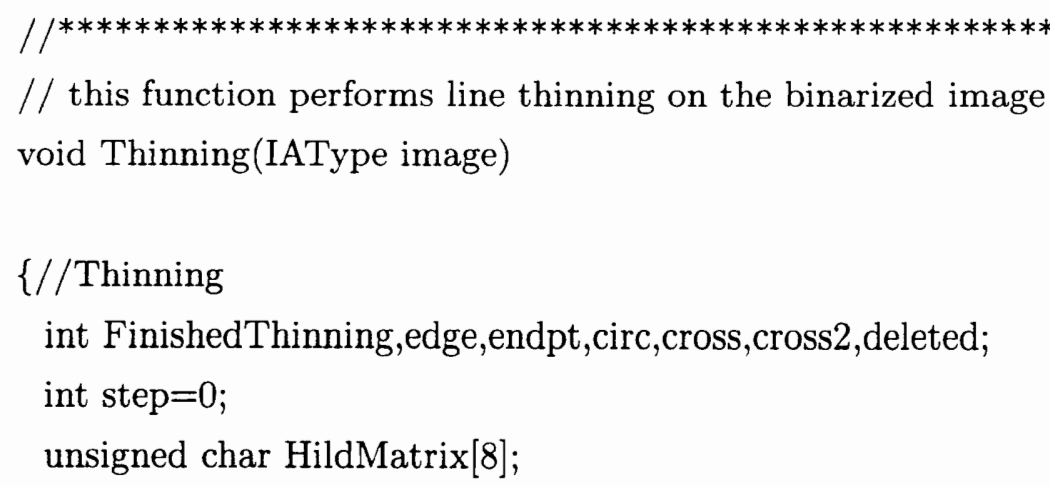

// this function performs line thinning on the binarized image void Thinning(IAType image)

\section{\{//Thinning}

int FinishedThinning,edge,endpt,circ,cross,cross2,deleted;

int step $=0$;

unsigned char HildMatrix[8];

$\operatorname{do}\{/ /$ while not done thinning

FinishedThinning = true;

deleted $=0$;

step++;

for (int $\mathrm{y}=1 ; \mathrm{y}<$ HEIGHT; ++ )

for (int $\mathrm{x}=1 ; \mathrm{x}<$ WIDTH; + + $)$

$\{/ /$ for $\mathrm{x}$

if(image $[x][y]==1)$

$\{/ /$ if pixel white

edge $=0$;

endpt $=0$;

$\operatorname{circ}=0$;

cross $=0$;

$\operatorname{cross} 2=0$;

HildMatrix $[0]=$ image $[\mathrm{x}][\mathrm{y}-1]$;

HildMatrix $[1]=$ image $[x+1][y-1]$;

HildMatrix $[2]=$ image $[x+1][y]$;

HildMatrix $[3]=$ image $[x+1][y+1]$;

HildMatrix $[4]=$ image $[\mathrm{x}][\mathrm{y}+1]$;

HildMatrix $[5]=$ image $[x-1][y+1]$;

HildMatrix $[6]=$ image $[x-1][y]$;

HildMatrix $[7]=$ image[x-1][y-1]; 


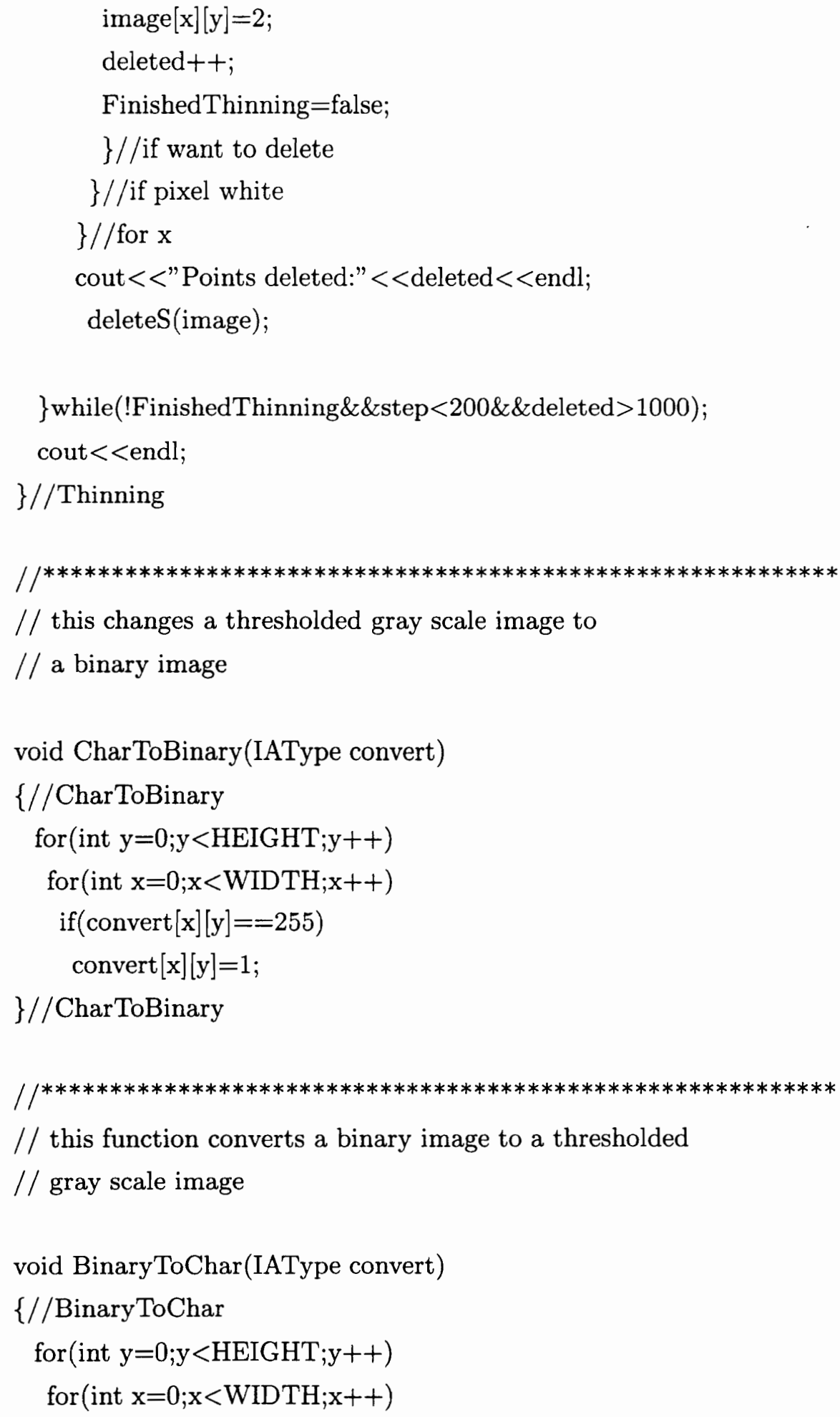




$$
\begin{aligned}
& \text { if }(\operatorname{convert}[\mathrm{x}][\mathrm{y}]==1) \\
& \operatorname{convert}[\mathrm{x}][\mathrm{y}]=255 \\
& \text { \}//BinarytoChar }
\end{aligned}
$$

$/{ }^{*}$

void deleteS(IAType del)

$\{/ /$ deleteS

$$
\begin{aligned}
& \text { for }(\text { int } y=0 ; y<\text { HEIGHT;y++) } \\
& \text { for }(\text { int } x=0 ; x<\text { WIDTH; } x++) \\
& \operatorname{if}(\operatorname{del}[x][y]==2) \\
& \quad \operatorname{del}[x][y]=0 \\
& \} / / \text { deleteS }
\end{aligned}
$$

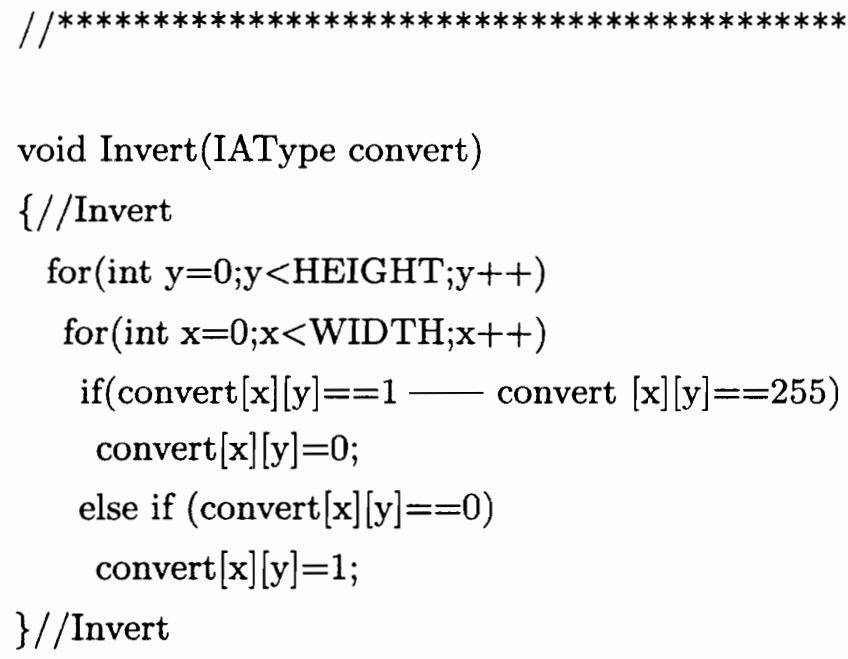




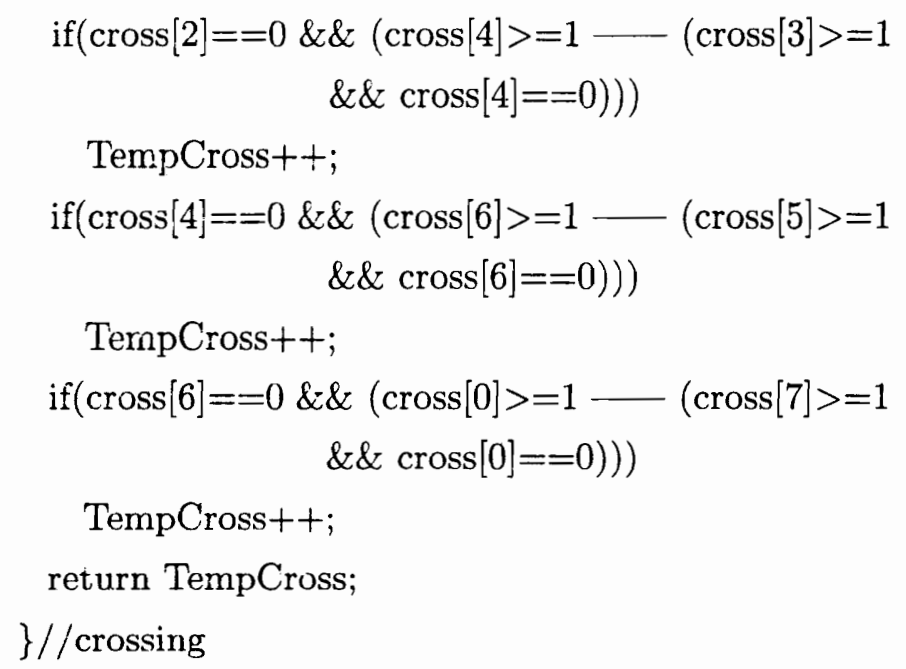

void ScaleImage(IAType image,IAType M, unsigned int SCol, unsigned int SRow, unsigned int SWidth, unsigned int SHeight, double ScaleH, double ScaleV, unsigned int DCol, unsigned int DRow, unsigned int Interpolate, int h1x, int vly)

$/{ }^{*}$ Scale image takes the image and distorts it to the users specifications*/

$\{/ /$ ScaleImage

unsigned int DestWidth, DestHeight;

unsigned int $\mathrm{PtA}, \mathrm{PtB}, \mathrm{PtC}, \mathrm{PtD}$, PixelValue;

unsigned int SPixelColNum, SPixelRowNum, DestCol, DestRow;

double SPixelColAddr, SPixelRowAddr;

double ColDelta, RowDelta;

double ContribFromAandB, ContribFromCandD;

DestWidth $=$ ScaleH*SWidth +0.5

DestHeight $=\mathrm{ScaleV}^{*}$ SHeight +0.5 ; 


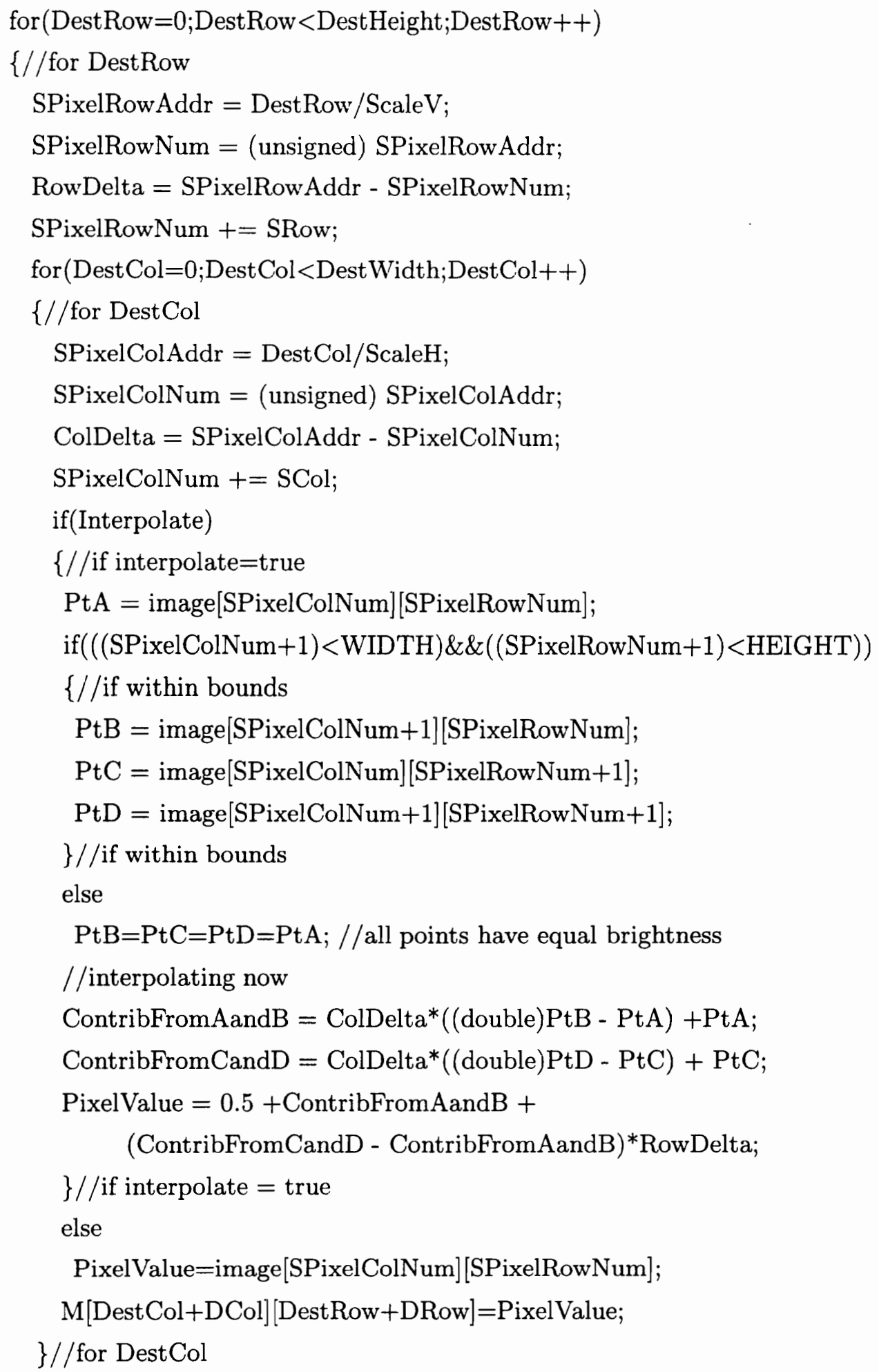




$$
\begin{aligned}
& \text { \}// for DestRow } \\
& \text { for (int } y=0 ; y<\text { HEIGHT; }++) \\
& \quad \text { for }(\text { int } x=0 ; x<\text { WIDTH } ; x++) \\
& \quad \text { image }[\mathrm{x}][\mathrm{y}]=\mathrm{M}[\mathrm{x}][\mathrm{y}]
\end{aligned}
$$

\}//ScaleImage

$/ / * * *+2$

// This function takes only the portion of the image within the given

// circle, defined by the origin and the radius. This is then dumped to the $/ /$ buffer

void MaskImage(IAType image,IAType buffer,int OriginY,int OriginX,int $r$, int $\mathrm{h} 1 \mathrm{x}$, int $\mathrm{v} 1 \mathrm{y})$

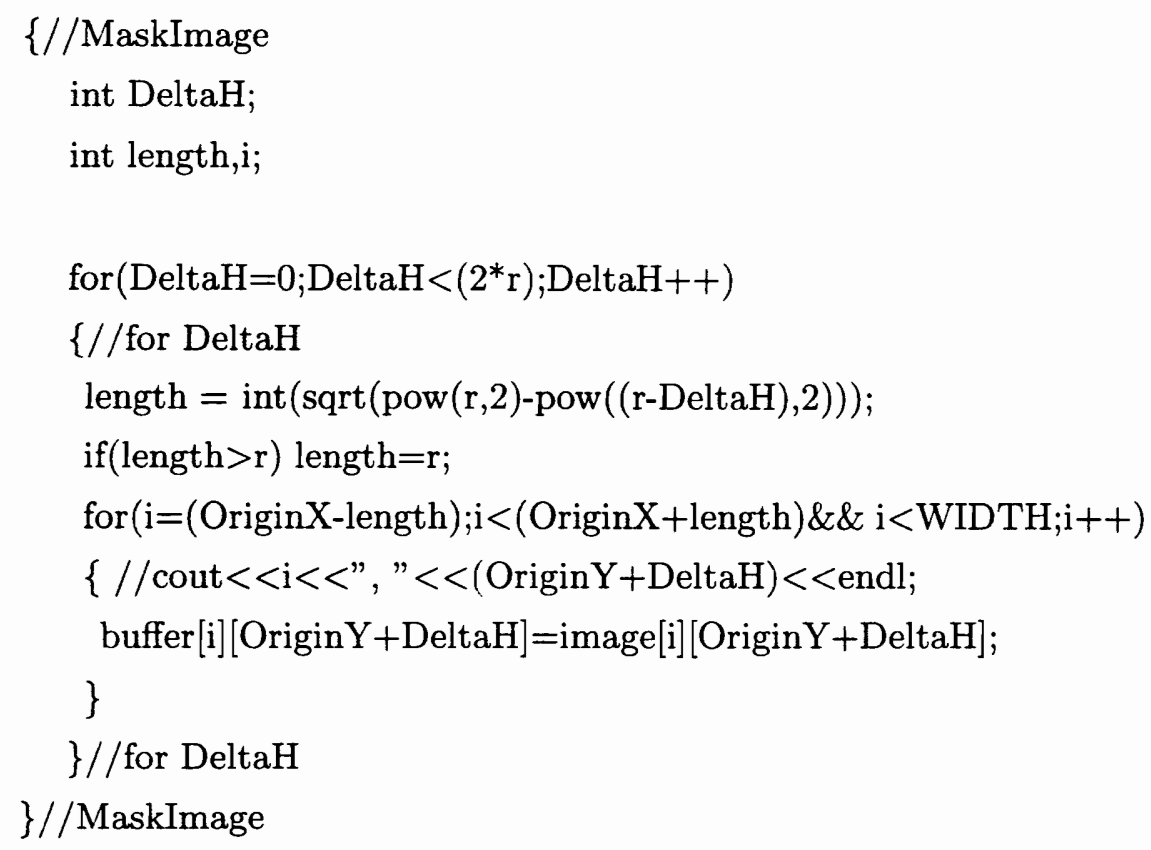

// This function shifts the center of the interferogram to the upper // right edge. This helps ensure that when the image is stretched 
$/ /$ it will not cross the image boundary

void ShiftImage(IAType image, IAType buffer, int h1x, int v1y)

$\{/ /$ ShiftImage

int $\mathrm{x}, \mathrm{y}, \mathrm{XShift}, \mathrm{YShift}$;

$$
\begin{aligned}
& \text { for }(\mathrm{y}=\mathrm{v} 1 \mathrm{y} ; \mathrm{y}<\text { HEIGHT; }++ \text { ) } \\
& \text { for }(\mathrm{x}=\mathrm{h} 1 \mathrm{x} ; \mathrm{x}<\text { WIDTH } ; \mathrm{x}++) \\
& \{/ / \text { for } \mathrm{x} \\
& \mathrm{XShift}=\mathrm{x}-\mathrm{h} 1 \mathrm{x}+12 \text {; } \\
& \text { YShift }=y-v 1 y+12 \text {; } \\
& \text { if }(\text { XShift }<12) \text { XShift }=12 \text {; } \\
& \text { if }(\text { YShift }<12) \text { YShift }=12 \text {; } \\
& \text { buffer }[\mathrm{x}-\mathrm{h} 1 \mathrm{x}+12][\mathrm{y}-\mathrm{v} 1 \mathrm{y}+12]=\text { image }[\mathrm{x}][\mathrm{y}] \text {; } \\
& \text { \}//for } \mathrm{x} \\
& \text { for }(y=0 ; y<\text { HEIGHT; }++) \\
& \text { for }(x=0 ; x<\text { WIDTH; } x++) \\
& \text { image }[\mathrm{x}][\mathrm{y}]=\text { buffer }[\mathrm{x}][\mathrm{y}] \text {; }
\end{aligned}
$$

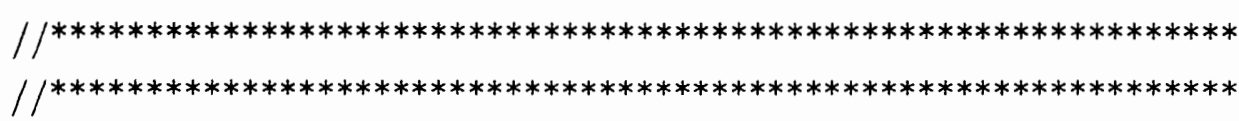

\section{// MAIN PROGRAM}

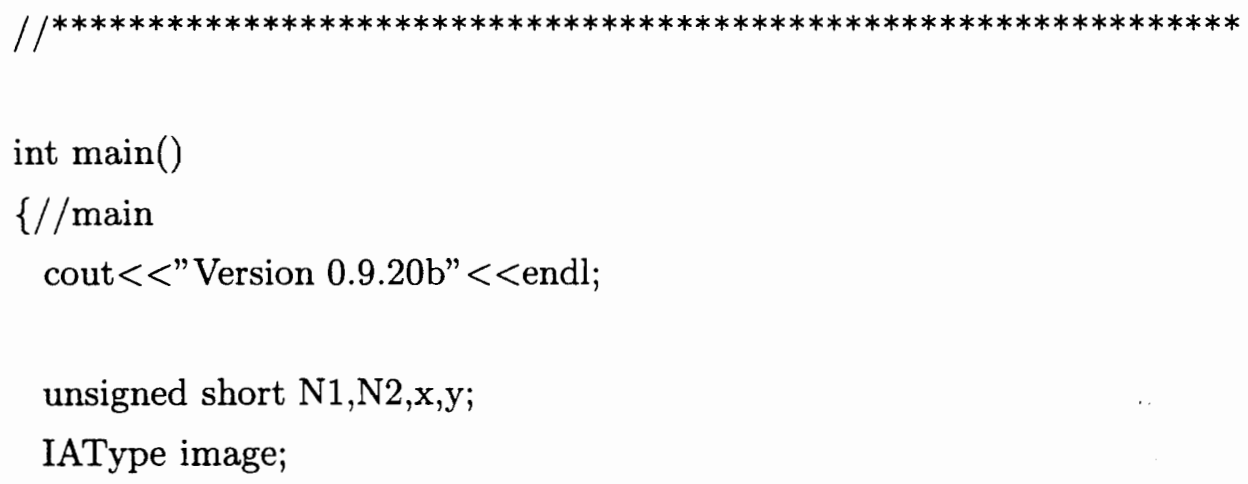


IAType buffer $=\{0\}$;

unsigned int SCol,SRow,SWidth,SHeight;

double ScaleH,ScaleV;

unsigned int DCol,DRow,Interpolate;

int $h 1 x, h 1 y, h 2 x, h 2 y, v 1 x, v 1 y, v 2 x, v 2 y$, OriginX, Origin $Y, r$;

$\mathrm{SCol}=1$;

SRow $=1$;

SWidth $=512$;

SHeight $=480$;

$\mathrm{DCol}=0$;

DRow $=0$;

Interpolate $=$ true;

cout $<<$ "Input $x$ coordinate of $h 1:$ : $\operatorname{cin}>>\mathrm{h} 1 \mathrm{x}$;

cout $<<$ "Input y coordinate of h1: "; $\operatorname{cin}>>\mathrm{h} 1 \mathrm{y}$;

$\mathrm{h} 2 \mathrm{y}=\mathrm{h} 1 \mathrm{y}$;

cout $<<$ "Input $x$ coordinate of $h 2:$ "; $\operatorname{cin}>>h 2 x$;

cout $<<$ "Input $x$ coordinate of $v 1$ : "; $\operatorname{cin}>>\mathrm{v} 1 \mathrm{x}$;

cout $<<$ "Input y coordinate of $\mathrm{v} 1:$ "; $\operatorname{cin}>>\mathrm{v} 1 \mathrm{y}$;

$\mathrm{v} 2 \mathrm{x}=\mathrm{v} 1 \mathrm{x}$;

cout $<<$ "Input y coordinate of $\mathrm{v} 2$ : "; $\operatorname{cin}>>\mathrm{v} 2 \mathrm{y}$;

$\mathrm{r}=\operatorname{int}(\operatorname{abs}(\mathrm{v} 1 \mathrm{y}-\mathrm{v} 2 \mathrm{y}) / 2)$;

$\mathrm{ScaleH}=1.21$

ScaleV=1;

Origin $Y=12$;

Origin $\mathrm{X}=12 * \mathrm{ScaleH}+\mathrm{r}$;

cout $<<$ "Scale change will be: $"<<$ ScaleH $<<$ endl;

cout $<<$ "Input Size of sampling square for image filtering: ";

$\operatorname{cin}>>\mathrm{N} 1$

cout $<<$ "Input size of sampling square for binarization: ";

$\operatorname{cin}>>\mathrm{N} 2$; 
if $((\mathrm{N} 1$

if $((\mathrm{N} 2$

ReadArray(image);

ShiftImage(image, buffer,h1x,v1y);

printf("Scaling image...\n");

ScaleImage(image,buffer,SCol,SRow,SWidth,SHeight,ScaleH,ScaleV, DCol,DRow,Interpolate,h1x,v1y);

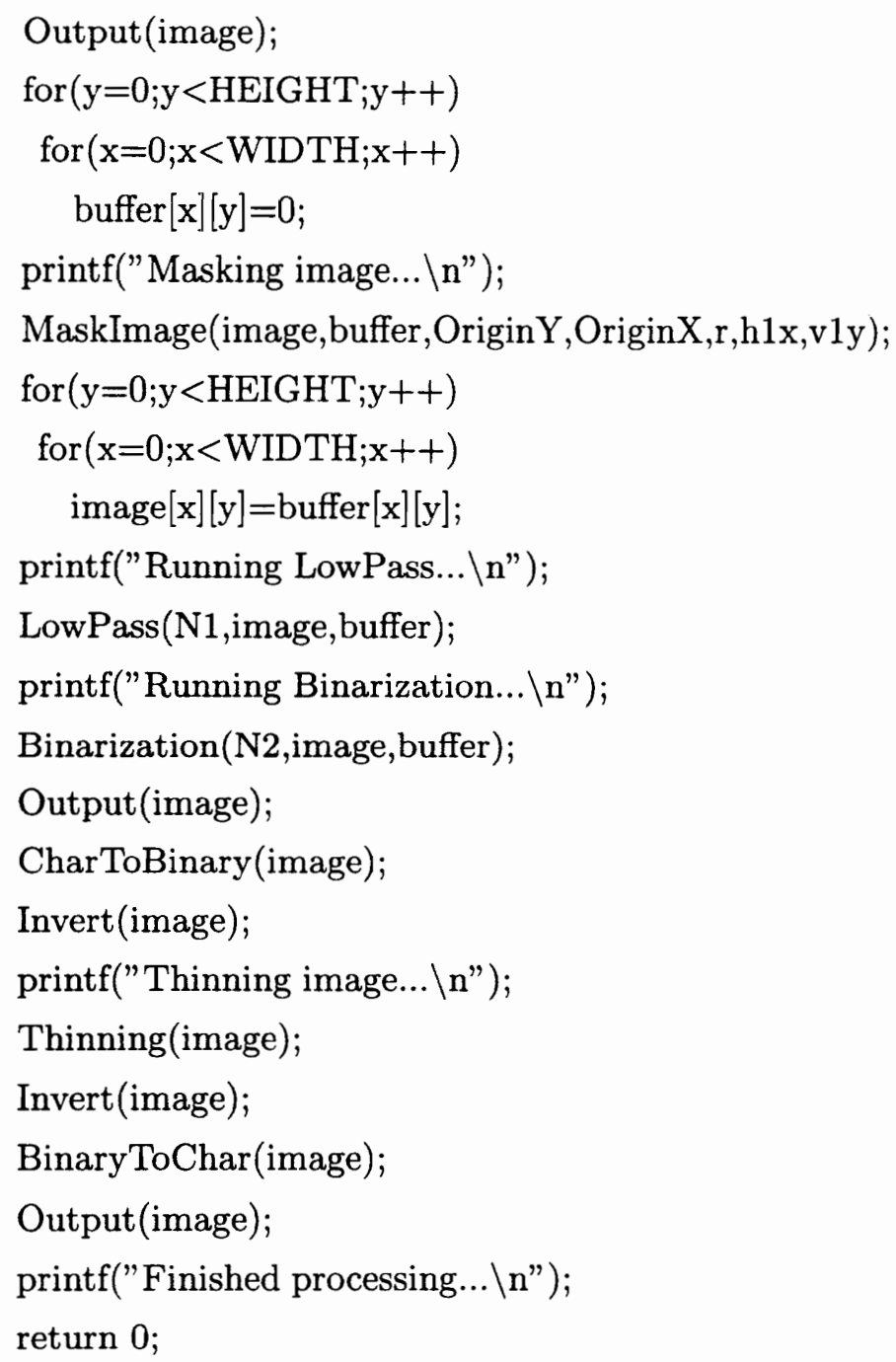




\section{Appendix F}

\section{Sitc.cpp}

$/{ }^{*}$ This programs takes the stretched fringe files and outputs an image shifted DeltaX and DeltaY, based on user input values for the pre-defined "middle point" */

\#include $<$ iostream.h $>$

\#include $<$ math.h $>$

\#include $<$ stdio.h $>$

\#include $<$ stdlib.h $>$

const unsigned short WIDTH $=512$;

const unsigned short HEIGHT $=480$;

const unsigned short true $=1$;

const unsigned short false $=0$;

typedef unsigned char IAType[WIDTH][HEIGHT];

void ReadArray(IAType image)

$\{/ /$ ReadArray

FILE * graphic;

char filename[30];

unsigned char buf[1]; 
short Done $=0$;

do

$\{/ /$ do while not a file

cout $<<$ "Filename: ";

$\operatorname{cin}>>$ filename;

if $($ (graphic $=$ fopen $($ filename "rb" $)) !=$ NULL $)$

Done $=1$;

\}while(!Done);

for (short $j=0 ; j<$ HEIGHT; ++ )

for (short $\mathrm{i}=0 ; \mathrm{i}<\mathrm{WIDTH} ; \mathrm{i}++$ )

\{

fread(buf,1,1,graphic);

image $[\mathrm{i}][\mathrm{j}]=$ buf $[0]$;

\}

fclose(graphic);

\}//ReadArray

void Output(IAType image)

$\{/ / O u t p u t$

FILE *OutStream;

char filename[30];

cout $<<$ "Output filename: ";

cin $>>$ filename;

OutStream = fopen(filename, "w"); 


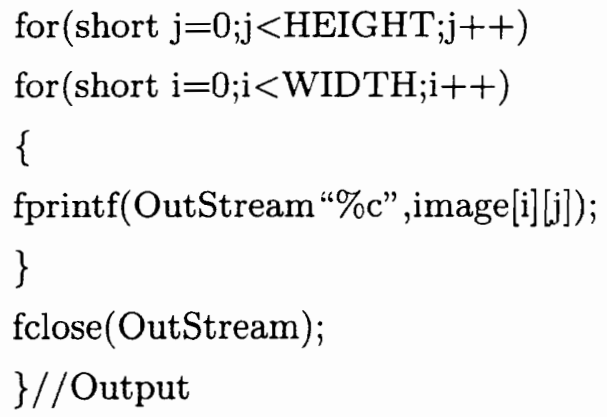

DeltaX $=256-\mathrm{x}$;

DeltaY $=240-y$;

for $(j=0 ; j<$ HEIGHT $; j++)$

for $(i=0 ; i<$ WIDTH; $i++)$

buffer $[\mathrm{i}][\mathrm{j}]=255$;

for $(j=0 ; j<$ HEIGHT $; j++)$

for $(\mathrm{i}=0 ; \mathrm{i}<\mathrm{WIDTH} ; \mathrm{i}++)$

$\{/ /$ for $\mathrm{i}$

if $((\mathrm{i}+$ DeltaX $)<$ WIDTH \&\& $(i+$ DeltaX $)>0)$

if $((j+$ DeltaY $)<$ HEIGHT $\& \&(j+$ DeltaY $)>0)$; 
buffer $[i+$ DeltaX $][j+$ DeltaY $]=$ image $[i][j]$;

\}$/ /$ for i

Output(buffer);

\}//main 


\section{Appendix G}

\section{Flip.cpp}

$/^{*}$ This program takes the loaded images and flips it along the horizontal axis. */

\#include<iostream.h>

\#include $<$ stdio.h $>$

\#include<stdlib.h>

const unsigned short WIDTH $=512$;

const unsigned short HEIGHT $=480$;

const unsigned short true $=1$;

const unsigned short false $=0$;

typedef unsigned char IAType[WIDTH][HEIGHT];

void ReadArray(IAType image)

$\{/ /$ ReadArray

FILE * graphic;

char filename[30];

unsigned char buf[1]; 
short Done $=0$;

do

$\{/ /$ do while not a file

cout $<<$ "Input Filename: ";

cin >>filename;

if $(($ graphic $=$ fopen(filename "rb" $)) !=$ NULL $)$

Done $=1$;

\}while(!Done);

for (short $j=0 ; j<$ HEIGHT $;++$ )

for (short $\mathrm{i}=0 ; \mathrm{i}<\mathrm{WIDTH} ; \mathrm{i}++$ )

\{

fread(buf,1,1,graphic);

image $[\mathrm{i}][\mathrm{j}]=$ buf $[0]$;

\}

fclose(graphic);

\}//ReadArray

void Output(IAType image)

$\{/ /$ Output

FILE *OutStream;

char filename[30];

cout $<<$ "Output filename: ";

cin $>>$ filename;

OutStream = fopen(filename"w"); 


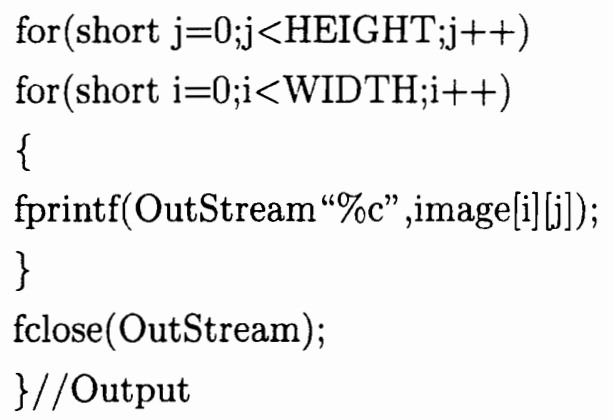

IAType buffer,image;

ReadArray(image);

for $(j=0 ; j<$ HEIGHT $;++)$

for $(\mathrm{i}=0 ; \mathrm{i}<\mathrm{WIDTH} ; \mathrm{i}++)$

buffer $[\mathrm{i}][\mathrm{j}]=255$;

for $(j=0 ; j<$ HEIGHT $;++)$

for $(\mathrm{i}=0 ; \mathrm{i}<\mathrm{WIDTH} ; \mathrm{i}++)$

$\{/ /$ for $\mathrm{i}$

buffer [i] [j] =image[i][HEIGHT-j];

\}$/ /$ for $i$

Output(buffer);

\}//main 


\section{Appendix $\mathbf{H}$ \\ ProcFin.cpp}

//Program ProcFin.cpp

$I^{*}$ This code is used to reduce interferometric images.

Given a thinned fringe flow and noflow images at all

three zones (both horizontally and vertically shifted)

and a reference density field, this code returns the stepped density field results.*/

\#include $<$ iostream.h $>$

\#include $<$ iomanip.h $>$

\#include $<$ fstream.h $>$

\#include $<$ stdio.h $>$

\#include < math.h $>$

\#include <stdlib.h $>$

\#define WIDTH 512

\#define HEIGHT 480

\#define true 1

\#define false 0

\#define DEFAULT -1 
\#define dhx_size 44

\#define dhy_size 230

\#define dhx_max 30

\#define dhy_max 218

/*Initalize Variables*/

ofstream buf;

const double $\mathrm{L}=0.0508$;

const double $\mathrm{k}=3 \mathrm{e}-4$;

const double Ro_ref $=1.1113$;

const double lambda_0 $=632.8^{*}(1 \mathrm{e}-9)$;

const double $\mathrm{n} \_0=1.0002506$;

$/{ }^{*}$ Computes the constant $\mathrm{K}$ required for the interferogram reduction density formula*/

const double $\mathrm{K}=\left(\left(\mathrm{L}^{*} \mathrm{k}\right) /\left(\mathrm{n} \_0^{*}\right.\right.$ Ro_ref $\left.)\right)$;

/*Type and define required variables functions and arrays*/

typedef unsigned char IAType[WIDTH][HEIGHT];

typedef float CountArray[WIDTH][HEIGHT]; 
typedef float DTypeH[dhx_size][dhy_size];

struct Holder

$\{/ /$ Holder

short XCoor;

short YCoor;

\};//Holder

int LastStep;

void HLoadImages(char NFName[],char FFName[], char DFName[]);

void HCountFringes(IAType image,CountArray fringes);

void HInterpolate(CountArray fringe);

void Output(CountArray current);

void IntOutput(CountArray current, char filename[]);

void ReadArray(IAType image);

void LoadDensity(DTypeH DensArray);

void HStudyStepRight(CountArray NF, CountArray FF, CountArray DF,

DTypeH DensArray,Holder\& Start,int max_x,int max_y);

void HStudyStepRightB(CountArray NF, CountArray FF, CountArray DF,

DTypeH DensArray,Holder\& Start,int max_x,int max_y);

void HStudyStepLeft(CountArray NF, CountArray FF, CountArray DF,

DTypeH DensArray,Holder\& Start,int max_x,int max.y);

void DensityInterpolate(DTypeH DensArray);

void Threshold(IAType image);

void LoadBuffer(CountArray ca,char filename[]);

void PutBuffer(CountArray ca,char filename[]);

void LoadHKnown(CountArray ca, DTypeH known,Holder Corners[]); 
int trunc(double $\mathrm{x})$;

$/ / * * * * * * * * * * * * * * * * * * * * * * * * * * * * * * * * * * * * * * * * * * * * * * * * * * * * * * * * * * * * * *$

//BEGIN FUNCTIONS

$/ /$

void HLoadImages(char NFName[], char FFName[], char DFName[]) ${ }^{*}$ HLoadImages is used to load the horizontally shifted interferograms into memory. The user is prompted for the file names of the flow and corresponding no-flow images. Once these have been properly selected this routine reads the arrays into the image buffer and counts the number of fringes. Once this is done the image buffer is then loaded with the second image and its fringes are counted. Next the routine interpolates the values of both of the fringe arrays. These values are then subtracted and stored in the passed variable "current". */ $\{/ /$ HLoadImages

IAType image;

CountArray NF,FF,DF; / NF=Noflow $\mathrm{FF}=$ Flow arrays $\mathrm{DF}=$ Density arrays int $\mathrm{x}, \mathrm{y}$;

for $(\mathrm{y}=0 ; \mathrm{y}<$ HEIGHT;y++)

for $(\mathrm{x}=0 ; \mathrm{x}<$ WIDTH; ++$)$

$\{/ /$ for $\mathrm{x}$

$\mathrm{NF}[\mathrm{x}][\mathrm{y}]=\mathrm{DEFAULT}$;

$\mathrm{FF}[\mathrm{x}][\mathbf{y}]=\mathrm{DEFAULT}$;

$\mathrm{DF}[\mathrm{x}][\mathrm{y}]=\mathrm{DEFAULT}$; 
\}$/ /$ for $\mathrm{x}$

cout $<<$ "HLoadImages" < <endl;

cout $<<$ "For no flow, ";

ReadArray(image);

Threshold(image);

HCountFringes(image,NF);

cout $<<$ "For flow, ";

ReadArray(image);

Threshold(image);

HCountFringes(image,FF);

HInterpolate(NF);

HInterpolate(FF);

PutBuffer(NF,NFName);

PutBuffer(FF,FFName);

PutBuffer(DF,DFName);

\}//HLoadImages

$/ / * *+$

void HCountFringes(IAType image,CountArray fringes)

${ }^{*}$ HCountFringes counts the number of fringes passed on a given line of travel.

The stepping procedure is across the horizontal and then down the verticle, since the slope of the fringe shift for horizontally shifted interferograms is close to zero degrees. This employs the same counting method as the VCountFringes routine. */

$\{/ /$ HCountFringes

cout $<<$ "HCountFringes" $<<$ endl; 
short $\mathrm{x}, \mathrm{y}$, FringeCount,LineCount;

for $(\mathrm{y}=0 ; \mathrm{y}<$ HEIGHT $; \mathrm{y}++)$

$\{/ /$ for $\mathrm{y}$

FringeCount $=0$;

LineCount $=0$;

for $(\mathrm{x}=1 ; \mathrm{x}<\mathrm{WIDTH} ; \mathrm{x}++)$

$\{/ /$ for $\mathrm{x}$

if(image $[\mathrm{x}-1][\mathrm{y}] !=$ image $[\mathrm{x}][\mathrm{y}])$

$\{/ /$ if image

FringeCount++;

if (FringeCount $>=2$ )

$\{/ /$ if fringe count

FringeCount $=0$;

LineCount++;

fringes $[\mathrm{x}][\mathrm{y}]=$ LineCount;

\}//if fringe count

\}$/ /$ if image

\}//for $\mathrm{x}$

\}$/ /$ for $y$

\}//HCountFringes

$/ / * *$

void HInterpolate(CountArray fringe)

${ }^{*}$ HInterpolate fills in the values between the actual fringes with values attained from a linear interpolation. Since the $\mathrm{m} 0$ here, we again go across the horizontal and down the vertical. This means 
that there are no skipped points and a bilinear interpolation in the end is unnecessary. */

$\{/ /$ HInterpolate

cout $<<$ "HInterpolate" $<<$ endl;

int $\mathrm{c}, \mathrm{x}, \mathrm{y}, \mathrm{AtG}$ apBeginning,DecrementCounter;

int $\mathrm{x} 0, \mathrm{y} 0, \mathrm{xc}, \mathrm{yc}, \mathrm{x} 1, \mathrm{y} 1$;

Holder value[514];

for $(\mathrm{y}=0 ; \mathrm{y}<$ HEIGHT; ++$)$

$\{/ /$ for $y$

AtGapBeginning $=$ false;

DecrementCounter $=0$;

for $(\mathrm{x}=0 ; \mathrm{x}<\mathrm{WIDTH} ; \mathrm{x}++)$

$\{/ /$ for $\mathbf{x}$

if(fringe $[\mathrm{x}][\mathrm{y}]$ !=DEFAULT \&\& !AtGapBeginning)

$\{/ /$ if fringe\&\&Gap

AtGapBeginning $=$ true;

value[0].XCoor $=\mathrm{x}$;

value[0]. YCoor $=y$;

DecrementCounter $=1$;

\}//if fringe\&\&Gap

else if(AtGapBeginning\&\&fringe $[\mathrm{x}][\mathrm{y}]==\mathrm{DEFAULT})$

$\{/ /$ if counting...DEFAULT

value[DecrementCounter].XCoor $=\mathrm{x}$;

value[DecrementCounter]. YCoor $=y$;

DecrementCounter++;

\}//if counting...DEFAULT

else if(AtGapBeginning\&\&fringe $[\mathrm{x}][\mathrm{y}] !=\mathrm{DEFAULT})$

$\{/ /$ if counting...!DEFAULT

$\mathrm{x} 0=$ value $[0] . \mathrm{XCoor}$;

y0=value [0].YCoor;

$\mathrm{x} 1=\mathrm{x}$;

$\mathrm{y} 1=\mathrm{y}$;

for $(\mathrm{c}=1 ; \mathrm{c}<$ DecrementCounter $; \mathrm{c}++)$ 


\section{$\{/ /$ for $\mathrm{c}$}

$\mathrm{xc}=$ value[DecrementCounter-c].XCoor;

$\mathrm{yc}=$ value $[$ DecrementCounter-c].YCoor;

if $(\operatorname{abs}(x c)>$ WIDTH $-\mathrm{xc}<0-\operatorname{abs}(\mathrm{yc})>$ HEIGHT $-\mathrm{yc}<0)$ break;

fringe $[\mathrm{xc}][\mathrm{yc}]=$ fringe $[\mathrm{x} 0][\mathrm{y} 0]+$

((fringe[x1][y1]-fringe[x0] [y0])*double( $(x c-x 0) /$ double $(\mathrm{x} 1-\mathrm{x} 0))$;

\}$/ /$ for c

DecrementCounter $=1$;

value[0].XCoor $=\mathrm{x}$;

value[0].YCoor $=\mathrm{y}$;

\}//if counting...!DEFAULT

\}$/ /$ for $\mathrm{x}$

\}//for y

\}//HInterpolate

$/ /$

void Output(CountArray current)

$/^{*}$ Outputs the given double array to a user defined file */

$\{/ /$ Output

cout $<<$ "Output" $<<$ endl;

ofstream OutStream;

char filename[30];

cout<<"Output filename: ";

cin $>>$ filename;

OutStream.open(filename);

for (short $\mathrm{j}=0 ; j<$ HEIGHT $; j++$ ) 


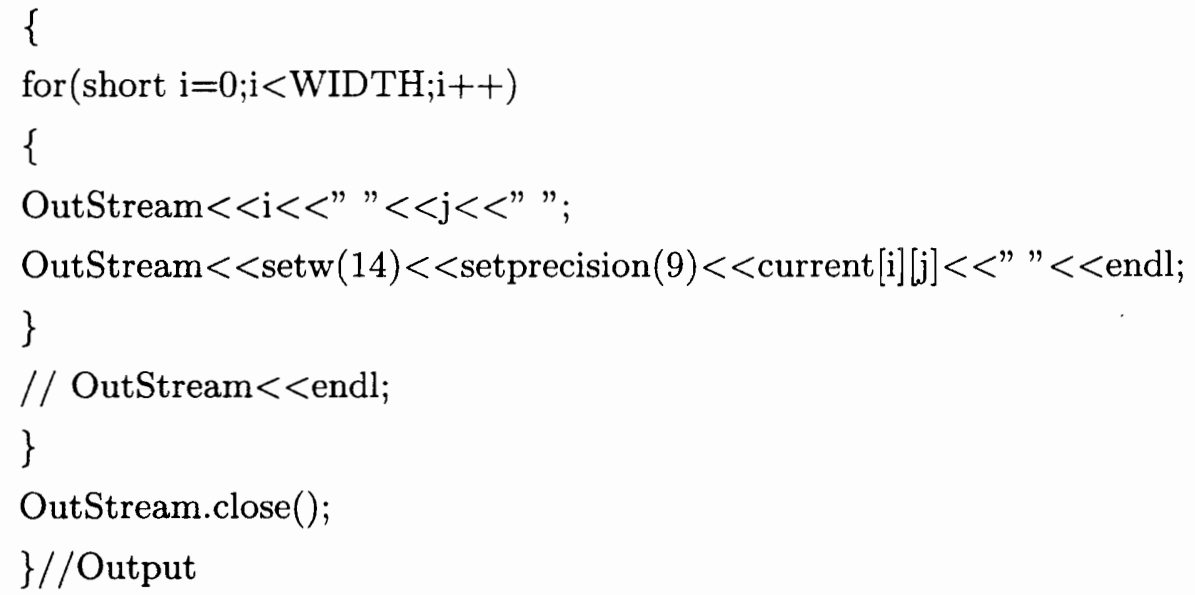


\}

out.close();

\}//IntOutput

$1 /$

void ReadArray(IAType image)

$/{ }^{*}$ Reads in an image from a user defined file */

$\{/ /$ ReadArray

cout $<<$ "ReadArray" $<<$ endl;

FILE *graphic;

char filename[30];

unsigned char buf[1];

short Done $=0$;

do

$\{/ /$ do while not a file

cout $<<$ "Input Filename: ";

cin $>>$ filename;

if((graphic=fopen(filename,"rb"))!=NULL)

Done $=1$;

\}while(!Done);

for $($ short $\mathrm{j}=0 ; \mathrm{j}<$ HEIGHT $; \mathrm{j}++$ )

for (short $\mathrm{i}=0 ; \mathrm{i}<\mathrm{WIDTH} ; \mathrm{i}++$ )

\{

fread(buf,1,1,graphic);

image $[\mathrm{i}][\mathrm{j}]=$ buf $[0]$;

\} 
fclose(graphic);

\}//ReadArray

$/ / * * * * * * * * * * * * * * * * * * * * * * * * * * * * * * * * * * * * * * * * * * * * * * * * * * * * * * * * * * * * * * * * *$

void LoadDensity(DTypeH DensArray)

${ }^{*}$ Prompts the user to fill in the value for the measured density points within the initial known density area. Once these points are entered a bilinear interpolation is used to fill in the rest of the values. */ $\{/ /$ LoadDensity

ifstream input;

double $\mathrm{x}, \mathrm{y}$,density;

input.open("240density.dat");

while(input)

\{

input $>>x>>y>>$ density;

if $\left(x<=d h x \_s i z e ~ \& \&(x-5)>=0 \& \& y<=d h y \_s i z e \& \&(y-5)>=0\right)$

DensArray[trunc(x)-5][trunc(y)-5]=density; //initial pt is $(5,5)$

\}//while

input.close();

\}//LoadDensity

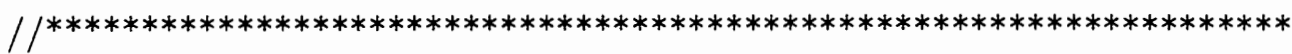

void HStudyStepRight(CountArray NF, CountArray FF, CountArray DF, 
DTypeH DensArray, Holder\& Start,int max_x,int max_y)

$/{ }^{*}$ Calculates the density from the known density field to the field of study to the right density field. This applies the equations and assumption found in Kiss in the area of data processing of interferogram analysis */

$\{/ /$ HStudyStepRight

cout $<<$ "HStudyStepRight" $<<$ endl;

int $\mathrm{x} \_$known, $\mathrm{y}$ known;

double Ro_A1,Ro_A2,Ro_B1,Ro_B2,DeltaN,DeltaF;

for $\left(y \_\right.$known $=0 ; y$ known $<=$ max_y;y_known ++$)$

$\{/ /$ for $\mathrm{y}$ known

Ro_A1 = DensArray $[0][\mathrm{y}$ known $]$;

$\mathrm{DF}[$ Start.XCoor $]\left[\right.$ Start.YCoor $+\mathrm{y} \_$known $]=$Ro_A1;

Ro_A2 = DensArray $\left[\max \_x\right]\left[y \_k n o w n\right]$;

$\mathrm{DF}[$ Start.XCoor $+\max \mathrm{x}]\left[\right.$ Start.YCoor $+\mathrm{y} \_$known $]=$Ro_A2;

for $\left(\mathrm{x} \_\right.$known $=1 ; \mathrm{x} \_$known $<\max \_\mathrm{x} ; \mathrm{x} \_$known ++$)$

$\{/ /$ for $\mathrm{x}$ known

Ro_B1 = DensArray[x_known] [y_known];

$\mathrm{DF}\left[\right.$ Start.XCoor $+\mathrm{x} \_$known] $\left[\right.$Start.YCoor $+\mathrm{y} \_$known] $=$Ro_B1;

DeltaN $=\mathrm{NF}\left[\right.$ Start.XCoor $\left.+\max \_\mathrm{x}\right]\left[\right.$ Start.YCoor $+\mathrm{y} \_$known]

- NF[Start.XCoor $\left.+\max \_x+x \_k n o w n\right]\left[S t a r t . Y C o o r+y \_k n o w n\right] ;$

DeltaF $=$ FF $\left[\right.$ Start.XCoor $\left.+\max \_x\right]\left[\right.$ Start.YCoor $\left.+y \_k n o w n\right]$

- FF[Start.XCoor $+\max \times \mathrm{x}+\mathrm{x} \_$known] [Start.YCoor $+\mathrm{y} \_$known];

Ro_B2 = lambda_0/K*(DeltaF- DeltaN)-(Ro_A1-Ro_B1)+Ro_A2;

buf $<<$ (Start.XCoor $+\max \_\mathrm{x}+\mathrm{x} \_$known $)<<" ~ "<<\left(\right.$ Start.YCoor $+\mathrm{y} \_$known $)<<$

$"$ " $<<$ DeltaN $<<" ~ "<<$ DeltaF $<<" ~ "<<<$

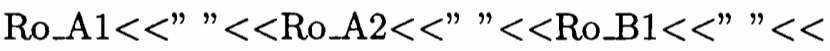


Ro_B2 $<<$ endl;

$\mathrm{DF}\left[\right.$ Start.XCoor $+\max \mathrm{x}+\mathrm{x} \_$known] $\left[\right.$Start.YCoor $+\mathrm{y} \_$known $]=$Ro_B2;

DensArray[x_known] $\left[\mathrm{y} \_\right.$known $]=$Ro_B2;

\}//for $\mathbf{x}$ known

Ro_B2 $=2 *$ DF $\left[\right.$ Start.XCoor $\left.+\max \_x+\mathrm{x} \_k n o w n-1\right]\left[\right.$ Start.YCoor $+\mathrm{y} \_$known]

- DF[Start.XCoor $+\max \times \mathrm{x}+\mathrm{x} \_$known-2][Start.YCoor $+\mathrm{y}$ known];

DensArray $\left[\mathrm{x} \_\right.$known $]\left[\mathrm{y} \_\right.$known $]=$Ro_B2;

$\mathrm{DF}\left[\right.$ Start.XCoor $+\max \mathrm{x}+\mathrm{x} \_$known] $\left[\right.$Start.YCoor $+\mathrm{y} \_$known $]=$Ro_B2;

DensArray $[0]\left[y \_\right.$known $]=$Ro_A2;

\}//for y_known

Start.XCoor $=$ Start.XCoor $+\max \_$;

// Start.YCoor = Start.YCoor; //just for clarity since only moving horizontal

\}//HStudyStepRight

void HStudyStepRightB(CountArray NF, CountArray FF, CountArray DF, DTypeH DensArray, Holder\& Start,int max_x,int max_y)

$/^{*}$ Calculates the density from the known density field to the field of study to the right density field. This applies the equations and assumption found in Kiss in the area of data processing of interferogram analysis */

$\{/ /$ HStudyStepRight 
cout $<<$ "HStudyStepRight" $<<$ endl;

int $\mathrm{x} \_$known, $\mathrm{y} \_$known;

double Ro_A1,Ro_A2,Ro_B1,Ro_B2,DeltaN,DeltaF;

for $\left(y \_\right.$known $=0 ; y \_$known $<=$max_y;y_known ++$)$

$\left\{/ /\right.$ for $\mathrm{y} \_$known

Ro_A1 = DensArray [0][y_known];

$\mathrm{DF}\left[\right.$ Start.XCoor] [Start.YCoor $+\mathrm{y} \_$known] $=$Ro_A1;

Ro_A2 = DensArray $\left[\max \_x\right]\left[y \_\right.$known $]$;

$\mathrm{DF}[$ Start.XCoor $+\max \mathrm{x}]\left[\right.$ Start.YCoor $+\mathrm{y} \_$known $]=$Ro_A2;

for $\left(\mathrm{x} \_\right.$known $=1 ; \mathrm{x} \_$known $<\max \_\mathrm{x} ; \mathrm{x} \_$known ++$)$

$\{/ /$ for $\mathrm{x}$ known

Ro_B1 = DensArray $\left[\mathrm{x} \_\right.$known $]\left[\mathrm{y} \_\right.$known $]$;

$\mathrm{DF}\left[\right.$ Start.XCoor $+\mathrm{x} \_$known] $\left[\right.$Start.YCoor $+\mathrm{y} \_$known] $=$Ro_B1;

DeltaN $=$ NF $\left[\right.$ Start.XCoor $\left.+\max \_x\right]\left[\right.$ Start.YCoor $+\mathrm{y} \_$known $]$

- NF[Start.XCoor $+\max \times \mathrm{x}+\mathrm{x} \_$known] [Start.YCoor $+\mathrm{y} \_$known];

DeltaF $=$ FF[Start.XCoor $\left.+\max \_x\right]\left[\right.$ Start.YCoor $\left.+y \_k n o w n\right]$

- FF[Start.XCoor +max_x+x_known][Start.YCoor+y_known];

Ro_B2 = lambda_0/K*(DeltaF-DeltaN)-(Ro_A1-Ro_B1)+Ro_A2;

$/ /$ buf $<<$ (Start.XCoor + max $\_x+$ x_known $)<<" ~ "<<($ Start.YCoor + y _known $)<<$

//" " $<<$ DeltaN $<<" ~ "<<$ DeltaF $<<" ~ "<<$

// Ro_A1<<" "<<Ro_A2<<" "<<Ro_B1<<" "<<

$/ /$ Ro_B2 $<<$ endl;

$\mathrm{DF}\left[\right.$ Start.XCoor $+\max \_\mathrm{x}+\mathrm{x} \_$known $]\left[\right.$Start.YCoor $+\mathrm{y} \_$known $]=$Ro_B2;

DensArray $\left[\mathrm{x} \_\right.$known $]\left[y \_k n o w n\right]=$ Ro_B2; 
\}//for $\mathrm{x}$ known

Ro_B2 $=2 *$ DF $\left[\right.$ Start.XCoor $\left.+\max \_x+x \_k n o w n-1\right]\left[S t a r t . Y C o o r+y \_k n o w n\right]$

- DF[Start.XCoor+max_x+x_known-2][Start.YCoor+y_known];

DensArray $\left[\mathrm{x} \_\right.$known $]\left[\mathrm{y} \_\right.$known $]=$Ro_B2;

$\mathrm{DF}\left[\right.$ Start.XCoor $+\max \_\mathrm{x}+\mathrm{x} \_$known $]\left[\right.$Start.YCoor $+\mathrm{y} \_$known $]=$Ro_B2;

DensArray $[0][y$ known $]=$ Ro_A2;

\}//for y known

Start.XCoor $=$ Start.XCoor $+\max \_$;

$/ /$ Start.YCoor $=$ Start.YCoor; //just for clarity since only moving horizontal

\}//HStudyStepRight

$/ / * *+1$

void HStudyStepLeft(CountArray NF, CountArray FF, CountArray DF, DTypeH DensArray, Holder\& Start,int max_x,int max_y)

/* Calculates the density from the known density field to the field of study to the left density field. This applies the equations and

assumption found in Kiss in the area of data processing of interferogram

analysis */

$\{/ /$ HStudyStepLeft

cout $<<$ "HStudyStepLeft" $<<$ endl;

int $\mathrm{x} \_$known,y_known; 
double Ro_A1,Ro_A2,Ro_B1,Ro_B2,DeltaN,DeltaF;

for $\left(y \_\right.$known $=0 ; y \_$known $<=$max_y;y_known ++$)$

$\{/ /$ for y known

Ro_B2 = DensArray[max $\left.\_x\right]\left[y \_k n o w n\right]$;

$\mathrm{DF}[$ Start.XCoor $+\max \mathrm{x}]\left[\right.$ Start.YCoor $+\mathrm{y} \_$known $]=$Ro_B2;

Ro_B1 = DensArray $[0]\left[y \_\right.$known $]$;

$\mathrm{DF}[$ Start.XCoor] [Start.YCoor +y known] = Ro_B1;

for $\left(\mathrm{x} \_\right.$known $=1 ; \mathrm{x} \_$known $<\max \_\mathrm{x} ; \mathrm{x} \_$known ++$)$

$\left\{/ /\right.$ for $\mathrm{x} \_$known

Ro_A2 = DensArray[max_x-x_known][y_known];

DF[Start.XCoor+max x-x_known] [Start.YCoor $\left.+y \_k n o w n\right]=R o \_A 2$;

DeltaN $=$ NF[Start.XCoor $+\max \times \mathrm{x}][$ Start.YCoor $+\mathrm{y}$ known]

$-\mathrm{NF}$ [Start.XCoor-x_known+max-x]

[Start.YCoor+y_known];

DeltaF $=$ FF $[$ Start.XCoor $+\max x][$ Start.YCoor $+y$ known $]$

-FF[Start.XCoor-x_known+max_x]

[Start.YCoor +y known];

Ro_A1 = lambda_0/K*(DeltaF-DeltaN) - Ro_B2 + Ro_B1 + Ro_A2;

$/ /$ buf $<<\left(\right.$ Start.XCoor $+\max \_\mathrm{x}+\mathrm{x} \_$known $)<<" ~ "<<$ (Start.YCoor $+\mathrm{y} \_$known $)<<$

//" " $<<$ DeltaN $<<" ~ "<<$ DeltaF $<<" ~ "<<<$

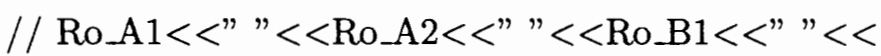

// Ro_B $2<<$ endl;

DF [Start.XCoor-x_known] [Start.YCoor +y known] $=$ Ro_A1;

DensArray[max_x-x_known] [y_known] $=$ Ro_A1; 
\}//for x_known

Ro_A1 $=2 *$ DF [Start.XCoor-x_known +1$]\left[\right.$ Start.YCoor $\left.+y \_k n o w n\right]$

- DF[Start.XCoor-x_known+2][Start.YCoor+y_known];

DensArray $[0]\left[\mathrm{y} \_\right.$known $]=$Ro_A1;

DF[Start.XCoor-x_known][Start.YCoor $\left.+y \_k n o w n\right]=R o \_A 1 ;$

DensArray[max_x $]\left[y \_k n o w n\right]=R o \_B 1 ;$

$\mathrm{DF}[$ Start.XCoor $]\left[\right.$ Start.YCoor $+\mathrm{y} \_$known $]=$Ro_B1;

\}// for y known

Start.XCoor $=$ Start.XCoor-max_x;

\}//HStudyStepLeft

$/ / * *+1$

void Threshold(IAType image)

${ }^{*}$ It was found that during file translations from the UNIX platforms to the Macintosh platform (which was used for manual image detail correction)

a background color of $10 \%$ black was given to all which pixels. Since

the background has to be white, this routine changes all pixels which are not black $(=0)$ to white pixels $(=255) * /$

$\{/ /$ Threshold

for (int $y=0 ; y<$ HEIGHT; ++ )

for (int $\mathrm{x}=0 ; \mathrm{x}<\mathrm{HEIGHT} ; \mathrm{x}++$ )

if(image $[x][y] !=0)$ 
image $[\mathrm{x}][\mathrm{y}]=255$;

\}//Threshold

void LoadBuffer(CountArray ca,char filename[])

$/{ }^{*}$ Loads the user requested buffer file into the "ca" array*/

$\{/ /$ LoadBuffer

ifstream in;

int $i, j$;

cout $<<$ "Loading buffer " $<<$ filename $<<$ endl;

in.open(filename);

for $(j=0 ; j<$ HEIGHT; $j++)$

for $(\mathrm{i}=0 ; \mathrm{i}<\mathrm{WIDTH} ; \mathrm{i}++)$

in $>$ ca $[\mathrm{i}][\mathrm{j}]$;

in.close ();

\}//LoadBuffer

$/ / * *$

void PutBuffer(CountArray ca,char filename[])

/*Puts the file of the buffer array "ca" into the selected filename*/

$\{/ /$ PutBuffer

ofstream out; 
int $i, j$;

cout $<<$ "Writing buffer " $<<$ filename $<<$ endl;

out.open(filename);

for $(j=0 ; j<$ HEIGHT $; j++)$

for $(\mathrm{i}=0 ; \mathrm{i}<\mathrm{WIDTH} ; \mathrm{i}++)$

out $<<$ ca $[\mathrm{i}][\mathrm{j}]<<$ endl;

out.close();

\}//PutBuffer

$/ / * * *$

void LoadHKnown(CountArray ca, DTypeH known,Holder Corners[])

$/^{*}$ Loads the area of the current buffer array into the Known array

using the passed variable "Corners" to define the area */

$\{/ /$ LoadHKnown

int $\mathrm{i}, \mathrm{j}, \mathrm{xi}, \mathrm{yi}, \mathrm{xf}, \mathrm{yf}, \mathrm{u}, \mathrm{v}$;

$\mathrm{xi}=$ Corners $[0] . \mathrm{XCoor}$

$\mathrm{yi}=$ Corners $[0]$.YCoor;

$\mathrm{xf}=$ Corners[2].XCoor;

$\mathrm{yf}=$ Corners[2].YCoor;

$\mathbf{u}=0$;

$\mathrm{v}=0$;

for $(j=y i ; j<=y f ; j++)$

$\{/ /$ for $\mathrm{j}$

$\mathrm{u}=0$; 
for $(i=x i ; i<=x f ; i++)$

$\{/ /$ for i

known $[\mathrm{u}][\mathrm{v}]=\mathrm{ca}[\mathrm{i}][\mathrm{j}]$;

$\mathrm{u}++$;

\}//for i

$\mathrm{v}++$;

\}//for $\mathrm{j}$

\}//LoadHKnown

$/ / * * * * * * * * * * * * * * * * * * * * * * * * * * * * * * * * * * * * * * * * * * * * * * * * * * * * * * * * * * * * * * * *$

int trunc(double $\mathrm{x}$ )

$/ /$ truncates off the decimal portion of $\mathrm{x}$ and returns its integer value $\{/ /$ trunc

double test;

test $=\mathrm{x}$-int $(\mathrm{x})$;

if (test $>=0$ )

return(int(x-test));

if $($ test $<0)$

$\operatorname{return}((\mathrm{x}-(\mathrm{x}-(\operatorname{int}(\mathrm{x})-1))))$;

\}$/ /$ trunc

$/ /$ 


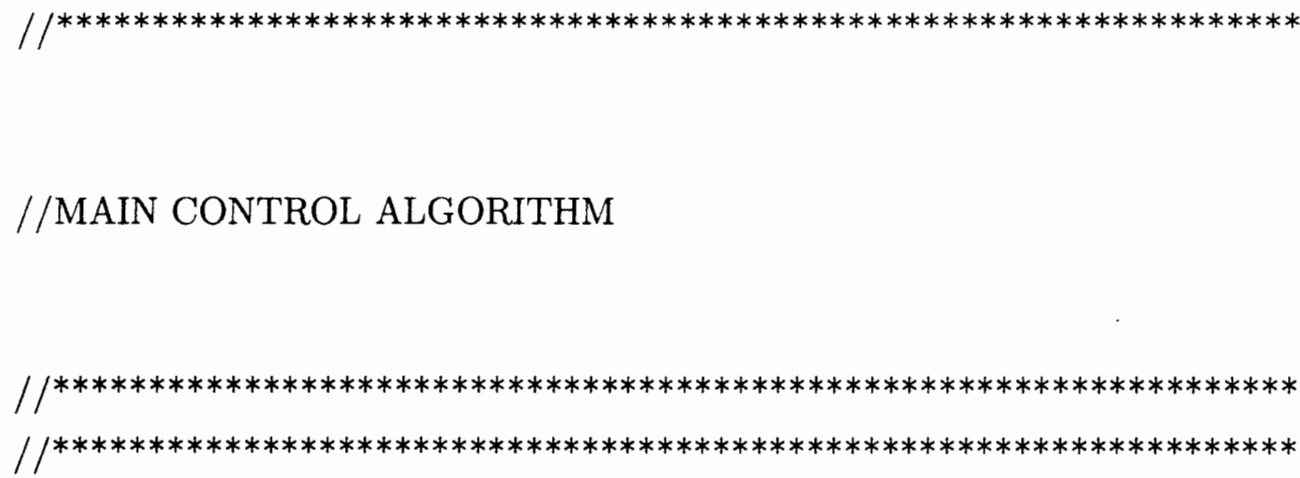

$/ / * * * * * * * * * * * * * * * * * * * * * * * * * * * * * * * * * * * * * * * * * * * * * * * * * * * * * * * * * * * * * * * * * * * *$

int main()

$\{/ /$ main

CountArray NBuf, FBuf, DBuf; //Noflow, Flow and Density Buffer DTypeH DensArray;

int step,xi,yi;

Holder Corners[4];

Holder Start;

int $\max \_x, \max \_y$;

Holder KnownArea[4];

buf.open("buffer.txt");

cout $<<$ "Version $0.20 \mathrm{~b} "<<$ endl;

cout $<<$ "For each prompt, enter first the NOFLOW then the FLOW images" $<<$ endl; cout $<<$ "For Position 1, horizontal: ";

HLoadImages("NH1.buf","FH1.buf","DH1.buf");

cout $<<$ "For Position 2, horizontal: "; 
HLoadImages("NH2.buf","FH2.buf","DH2.buf");

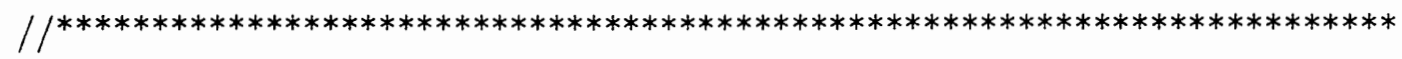

// begin processing images

$/ / * * * * * * * * * * * * * * * * * * * * * * * * * * * * * * * * * * * * * * * * * * * * * * * * * * * * * * * * * * * * * * * * * * * * * * * * * * * * * *$

// step to the left 6 iterations on image $\mathrm{H} 1$

LoadDensity(DensArray);

LoadBuffer(NBuf,"NH1.buf");

LoadBuffer(FBuf,"FH1.buf");

LoadBuffer(DBuf,"DH1.buf");

$\mathrm{xi}=202$;

$\mathrm{yi}=195$;

$\max \_\mathrm{x}=30$;

$\max _{-} y=218$;

Start. $\mathrm{XCoor}=\mathrm{xi}$;

Start.YCoor $=y i$

for $($ step $=0 ;$ step $<6 ;$ step ++$)$

$\{/ /$ for step

cout <<"Starting point: ("<<Start.XCoor $<<"$," <<Start.YCoor $<<$ endl;

HStudyStepLeft(NBuf,FBuf,DBuf,DensArray,Start,max_x,max_y);

\}//

$/ / * * * * * * * * * * * * * * * * * * * * * * * * * * * * * * * * * * * * * * * * * * * * * * * * * * * * * * * * * * * * * * * * * * * * * * *$

// step to the right seven iterations on image $\mathrm{H} 1$

$/ /$ corners of the zones are labeled from 0 to 3 , starting in 
// the upper left corner and proceeding clockwise.

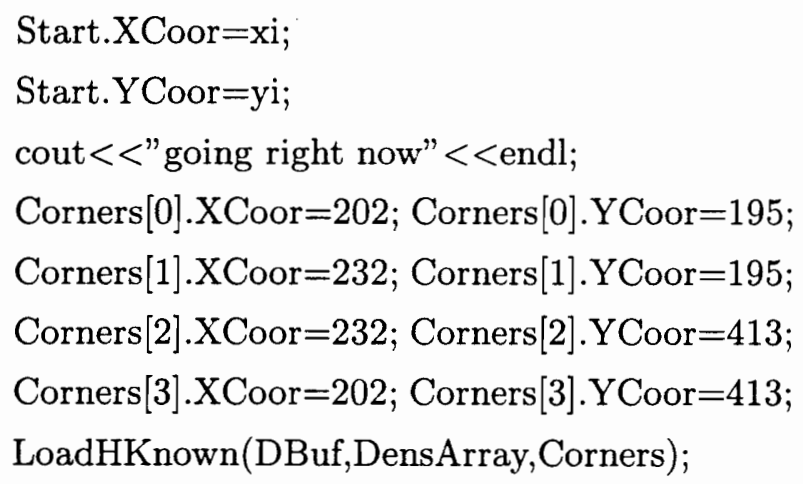

// here, in order to insure horizontal matching of the shock

// location the user is prompted for the location of the know

$/ /$ density field to be saved in the buffer

// step right seven iterations in image $\mathrm{H} 2$

KnownArea[0].XCoor $=338$; KnownArea[0].YCoor $=195$;

KnownArea[2].XCoor=368; KnownArea[2].YCoor $=413$;

LoadHKnown(DBuf,DensArray,KnownArea);

PutBuffer(NBuf,"NH1.buf"); 


\section{PutBuffer(FBuf,"FH1.buf"); \\ PutBuffer(DBuf,"DH1.buf"); \\ LoadBuffer(NBuf,"NH2.buf"); \\ LoadBuffer(FBuf,"FH2.buf"); \\ LoadBuffer(DBuf,"DH2.buf");}

cout $<<$ "Input the starting coordinates for the new image:" <<endl;

$\mathrm{xi}=246$;

$\mathrm{yi}=201$;

Start.XCoor $=\mathrm{xi}$;

Start. YCoor $=\mathrm{yi}$;

$\max \_x=30$;

$\max \_y=218$;

for $($ step $=0 ;$ step $<7 ;$ step ++$)$

$\{/ /$ for step

cout $<<$ "Starting point: (" $<<$ Start.XCoor $<<$ ", $<<$ Start.YCoor $<<$ endl;

HStudyStepRight(NBuf,FBuf,DBuf,DensArray,Start,max_x,max_y);

\}// for step

PutBuffer(DBuf,"DH2.buf");

//....................image processing complete ...//

//OutPut all buffer files with $\mathrm{x}, \mathrm{y}$ coordinates

// LoadBuffer(NBuf,"NH1.buf");

// LoadBuffer(FBuf,"FH1.buf");

LoadBuffer(DBuf,"DH1.buf");

// IntOutput(NBuf,"NH1.buf");

// IntOutput(FBuf,"FH1.buf"); 
IntOutput(DBuf,"DH1.buf");

// LoadBuffer(NBuf,"NH2.buf");

// LoadBuffer(FBuf,"FH2.buf");

LoadBuffer(DBuf,"DH2.buf");

// IntOutput(NBuf,"NH2.buf");

// IntOutput(FBuf,"FH2.buf");

IntOutput(DBuf,"DH2.buf");

buf.close();

\}$/ /$ main

$/ /$ / $* * * * * * * * * * * * * * * * * * * * * * * * * * * * * * * * * * * * * * * * * * * * * * * * * * * * * * * * * * * * * * * * * * * * * *$

$/ / * * * * * * * * * * * * * * * * * * * * * * * * * * * * * * * * * * * * * * * * * * * * * * * * * * * * * * * * * * * * * * * * * * * * * *$ 


\section{Appendix I}

\section{Raw Interferometric Data}

All of the interferograms taken by the author in the course of this research, both used and unused, are archived at Virginia Polytechnic Institute and State University. Due to the large number of images (over 60), it was decided that the inclusion of the images as figures in this dissertation would be combersome. Therefore, a DAT tape copy has been created, along with an file contaning an index of the images and information on the file formats, of all the interferograms taken during the final test series. The images are all presented in raw grayscale format and the resulting images are 512 by 480 pixels.

With the author's or the chairman's permission, a copy of the archived tape is avaliable from the Aerospace and Ocean Engineering Department of Virginia Polytechnic Institute and State University. 


\section{Vita}

The author, Angela L. Wesner, was born on November 24, 1970 in Portsmouth, Virginia. She graduated from Suffolk High School in 1988. Upon graduating from high school, the author came to Virginia Polytechnic Institute and State University to study Aerospace Engineering. While at Virginia Tech she also was a member of the Virginia Tech Corps of Cadets and the Virginia Tech Regimental Band, the "Highty Tighties". During her senior year as an undergraduate at Virginia Tech she attained the Regimental S4 position within the Corps and the rank of Cadet Major. Upon graduating in May, 1993 Summa Cum Laude with a Bachelor of Science in Aerospace Engineering (second in College of Engineering and first in her department), she remained at Virginia Tech in order to pursue a Doctoral degree in aerospace engineering. She is a member of both the AIAA and Phi Kappa Phi. Upon the completion of this work, the author plans to move to Columbia, Maryland where the she has accepted a postdoc position with the Applied Physics Laboratory of Johns Hopkins University in the area of stratified flow research.

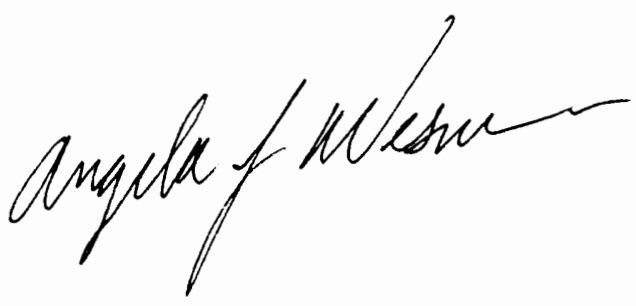

